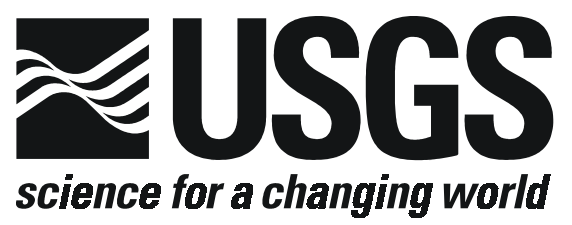

\title{
MAGNETOTELLURIC SURVEY TO LOCATE THE ARCHEAN/PROTEROZOIC SUTURE ZONE IN NORTH EASTERN NEVADA
}

\author{
By Jackie M. Williams and Brian D. Rodriguez
}

Any use of trade names is for descriptive purposes only and does not imply endorsement by the U.S. Government.

\section{U.S. Geological Survey Open-File Report 2004- 1215}

U.S. DEPARTMENT OF THE INTERIOR

U.S. GEOLOGICAL SURVEY 


\section{TABLE OF CONTENTS}

INTRODUCTION

MAGNETOTELLURIC METHOD

3

MAGNETOTELLURIC SURVEY

MAGNETOTELLURIC DATA

REFERENCES CITED 9

APPENDIX - MAGNETOTELLURIC DATA PLOTS 


\section{INTRODUCTION}

The Great Basin is part of the Basin and Range province covering a large part of Western United States. Many sedimenthosted gold deposits occur along linear trends in the Great Basin. The distribution and genesis of these deposits in the Great Basin is not fully understood. In general, most models agree that regional structures played an important role in the spatial distribution of these deposits (e.g. Arehart and others, 1993; Ilchik and Barton, 1997; Radtke, 1985; Shawe, 1991;

Sillitoe and Bonham, 1990; Tosdal, 1998). To investigate crustal structures that may be related to the genesis of gold deposits in the Great Basin, a regional south-north profile of magnetotelluric (MT) soundings was acquired in 2003 (Figure 1). Resistivity modeling of the MT data can be used to investigate buried structures or sutures that may have influenced subsequent tectonism, sedimentation, and regional fluid flow. The goal of this survey is to infer the location of the Archean/Proterozoic suture zone in north-eastern Nevada. The purpose of this report is to release the MT sounding data; no interpretation of the data is included.

\section{MAGNETOTELIURIC METHOD}

The magnetotelluric (MT) method is a passive surface geophysical technique, which uses the earth's natural electromagnetic fields to investigate the electrical resistivity structure of the subsurface. The resistivity of geologic units is largely dependent upon their fluid content, porosity, degree of fracturing, temperature, and conductive mineral content (Keller, 1989). Saline fluids within the pore spaces and fracture openings can reduce resistivities in a resistive rock matrix. Also, resistivity can be lowered by the presence of conductive clay minerals, carbon, and metallic mineralization. It is common for altered volcanic rocks to contain authigenic minerals that have resistivities ten times lower than those of the surrounding rocks (Nelson and Anderson, 1992). Increased temperatures cause higher ionic mobility and mineral activation energy, reducing rock resistivities significantly. Unaltered, unfractured igneous rocks are moderately to highly resistive (hundreds to thousands of ohm-m), whereas fault zones will show low resistivity (less than $100 \mathrm{ohm}-\mathrm{m}$ ) when they are comprised of rocks fractured enough to have hosted fluid transport and consequent mineralogical alteration (Eberhart-Phillips and others, 1995). Carbonate rocks are moderately to highly resistive (hundreds to thousands of ohm-m) depending upon their fluid content, porosity, fracturing, and impurities. Marine shales, mudstones, and clay-rich alluvium are normally very conductive (a few ohm-m to tens of ohm-m). Unaltered, metamorphic rocks (non-graphitic) are moderately to highly resistive (hundreds to thousands of ohm-m). Tables of electrical 
resistivity for a variety of rocks, minerals and geological environments may be found in Keller (1987) and Palacky (1987).

The MT method can be used to probe the crust from depths of tens of meters to depths of tens of kilometers (Vozoff, 1991). Natural variations of the Earth's magnetic and electric fields are measured and recorded at each MT station. The primary frequency bands used by the MT method are 10,000 $\mathrm{Hz}$ to $1 \mathrm{~Hz}$ from worldwide lightning activity and $1 \mathrm{~Hz}$ to $0.0001 \mathrm{~Hz}$ from geomagnetic micro-pulsations. The natural electric and magnetic fields propagate vertically in the earth because the very large resistivity contrast between the air and the earth causes a vertical refraction of both fields transmitted into the earth (Vozoff, 1972).

The natural electric and magnetic fields are recorded in two orthogonal, horizontal directions. The vertical magnetic field is also recorded. The resulting time-series signals are used to derive the tensor apparent resistivities and phases. First, the signals are converted to complex cross-spectra using fastFourier-transform (FFT) techniques. Then, least-squares, crossspectral analysis (Bendat and Piersol, 1971) is used to solve for a transfer function that relates the observed electric fields to the magnetic fields under the assumption that the Earth consists of a two-input, two-output, linear system with the magnetic fields as input and the electric fields as output. Prior to conversion to apparent resistivity and phase, the tensor is normally rotated into principal directions that correspond to the direction of maximum and minimum apparent resistivity. For a two-dimensional (2-D) Earth, the MT fields can be de-coupled into transverse electric (TE) and transverse magnetic (TM) modes; 2-D modeling is generally done to fit both modes. When the geology satisfies the 2-D assumption, the MT data for the TE mode is for the electric field parallel to geologic strike, and the data for the TM mode is for the electric field across strike. The MT method is well suited for studying complicated geological environments because the electric and magnetic relations are sensitive to vertical and horizontal variations in resistivity. The method is capable of establishing whether the electromagnetic fields are responding to subsurface terranes of effectively 1-, 2-, or 3-dimensions. An introduction to the MT method and references for a more advanced understanding are contained in Dobrin and Savit (1988) and Vozoff (1991).

\section{MAGNETOTELLURIC SURVEY}

Twenty-five stations roughly $10 \mathrm{~km}$ apart were collected starting north of Cobre, Nevada, then continuing south along Goshute Valley, Antelope Valley and Spring Valley ending just south of Highway 894 and Robinson Ranch. The station locations were chosen to cross the Archean/Proterozoic suture zone. The location of the suture zone is inferred from unpublished basement 
gravity maps (Grauch, 2003). Station locations were selected near roads for easy access and avoiding electrical noise, such as power lines. All stations were collected with a portable EMI MT-1 system (EMI, 1996). Horizontal electric fields were sensed using copper sulfate porous pots placed in an L-shaped, threeelectrode array with dipole lengths of $30 \mathrm{~m}$. The orthogonal, horizontal magnetic fields in the direction of the electric field measurement array were sensed using permalloy-cored induction coils. Frequencies sampled ranged from 70 to .009 Hz using single station recordings of both orthogonal horizontal components of the electric and magnetic fields, along with the vertical magnetic field.

The following table lists the twenty-five MT station locations as recorded using a global positioning system during field acquisition. Coordinates are referenced to the 1866 Clarke spheroid and North American 1927 Western United States datum. Longitude and latitude format is degrees:minutes:seconds. Universal Transverse Mercator units are in meters. Station elevation is given in meters. The accuracy of the $x, y$, and $z$ component is + or - 5 meters.

\begin{tabular}{|c|c|c|c|c|c|}
\hline Station & Longitude & Latitude & $\begin{array}{c}\text { UTM } \\
\text { North (m) }\end{array}$ & $\begin{array}{c}\text { UTM } \\
\text { East (m) }\end{array}$ & Elev (m) \\
\hline 1 & $-114: 25: 29$ & $41: 11: 30$ & $4,563,017$ & $11,715,975$ & 1911 \\
\hline 2 & $-114: 26: 55$ & $41: 02: 21$ & $4,546,044$ & $11,714,473$ & 1760 \\
\hline 3 & $-114: 28: 20$ & $40: 58: 52$ & $4,539,519$ & $11,712,676$ & 1727 \\
\hline 4 & $-114: 24: 36$ & $40: 52: 02$ & $4,527,032$ & $11,718,265$ & 1714 \\
\hline 5 & $-114: 21: 59$ & $40: 47: 13$ & $4,518,217$ & $11,722,222$ & 1723 \\
\hline 6 & $-114: 21: 21$ & $40: 41: 51$ & $4,508,329$ & $11,723,409$ & 1739 \\
\hline 7 & $-114: 20: 45$ & $40: 36: 45$ & $4,498,906$ & $11,724,549$ & 1730 \\
\hline 8 & $-114: 22: 14$ & $40: 31: 04$ & $4,488,338$ & $11,722,758$ & 1712 \\
\hline 9 & $-114: 20: 26$ & $40: 25: 37$ & $4,478,334$ & $11,725,620$ & 1727 \\
\hline 10 & $-114: 18: 00$ & $40: 20: 39$ & $4,469,259$ & $11,729,341$ & 1717 \\
\hline 11 & $-114: 19: 10$ & $40: 15: 00$ & $4,458,734$ & $11,727,997$ & 1734 \\
\hline 12 & $-114: 18: 25$ & $40: 09: 15$ & $4,448,121$ & $11,729,370$ & 1770 \\
\hline 13 & $-114: 17: 08$ & $40: 03: 51$ & $4,438,185$ & $11,731,515$ & 1794 \\
\hline 14 & $-114: 17: 30$ & $39: 58: 28$ & $4,428,231$ & $11,731,292$ & 1785 \\
\hline 15 & $-114: 20: 19$ & $39: 52: 50$ & $4,417,691$ & $11,727,593$ & 1730 \\
\hline 16 & $-114: 19: 57$ & $39: 47: 31$ & $4,407,858$ & $11,728,413$ & 1727 \\
\hline 17 & $-114: 19: 40$ & $39: 42: 18$ & $4,398,211$ & $11,729,110$ & 1861 \\
\hline 18 & $-114: 20: 30$ & $39: 36: 57$ & $4,388,278$ & $11,728,205$ & 1863 \\
\hline 19 & $-114: 22: 00$ & $39: 31: 21$ & $4,377,851$ & $11,726,369$ & 1722 \\
\hline 20 & $-114: 23: 11$ & $39: 26: 12$ & $4,368,302$ & $11,724,936$ & 1706 \\
\hline 21 & $-114: 21: 14$ & $39: 20: 49$ & $4,358,424$ & $11,728,034$ & 1721 \\
\hline 22 & $-114: 24: 16$ & $39: 15: 35$ & $4,348,615$ & $11,723,941$ & 1733 \\
\hline 23 & $-114: 24: 43$ & $39: 10: 55$ & $4,339,945$ & $11,723,548$ & 1770 \\
\hline 24 & $-114: 26: 01$ & $39: 04: 39$ & $4,328,289$ & $11,722,002$ & 1815 \\
\hline 25 & $-114: 30: 42$ & $38: 56: 50$ & $4,313,649$ & $11,715,649$ & 1811 \\
\hline
\end{tabular}




\section{MAGNETOTELLURIC DATA}

The recorded time-series data were transformed to the frequency domain and processed to determine a two-dimensional apparent resistivity and phase tensor at each site. Rotation of the impedance tensor to maximum and minimum directions allows for decoupling into the TE and TM modes.

Although true remote reference techniques were not used in our survey, we did sort cross-power files to select optimal signal-to-noise time series data sets (see Appendix).

The effects of near-surface resistivity anomalies cause "static shifts" (Sternberg et al., 1988) in the data. Static shift is significant in stations 17 and 25 and less significant in stations 6, 8, and 18. Cultural features can affect the response of the MT system. Fences, pipelines, communication lines, railways and other man-made conductors can contaminate the responses.

The figures in the Appendix represent the field-processed MT data for each station after the time series data were converted to the frequency domain as described above in the "Magnetotelluric Method" section.

For each station, eight separate plots are given:

1. Apparent Resistivity

2. Impedance Phase

3. Impedance Skew

4. Multiple Coherency

5. Impedance Polar Plots

6. Tipper Magnitude

7. Tipper Strike

8. $\mathrm{HzHx}$ and HzHy Coherency

Error bars on the Apparent Resistivity, Impedance Phase, Skew, Tipper Magnitude, and Tipper Strike plots represent probable errors within one standard deviation of the sample variance (Gamble and others, 1979).

Apparent resistivity is calculated from the ratio of the electric field strength magnitude over the magnetic field strength magnitude for a given frequency. The impedance phase is proportional to the slope of the apparent resistivity curve on a log-log plot, but from a baseline at -45 degrees (Vozoff, 1991). A measure of the dimensionality for MT data is provided by the impedance skew of the impedance tensor (Vozoff, 1972). If the effective measured resistivity response to the geology beneath a MT station is truly $1-D$ or $2-D$, then the skew will be zero. Both instrument and environmental sources of noise contribute to nonzero skew values, but are typically small (about 0.1) for relatively low noise level recordings. Higher skews (above 0.2) are an indication of either the resistivity response to 3-D geology or higher levels of noise. Local man-made electrical 
noise, such as power lines, power generators, moving vehicles and trains, produce an incoherent noise mainly affecting frequencies above $1 \mathrm{~Hz}$. Other man-made electrical noise, such as direct current electric trains and active cathodic protection of pipelines produce coherent electromagnetic signals mainly affecting frequencies below $1 \mathrm{~Hz}$.

In the survey area, noise from a number of small power lines and small moving vehicles was negligible at distances of $0.4 \mathrm{~km}$ and greater from the noise source. Power line levels were measured at each site and were typically less than 20 percent of the maximum recordable signals. Noise from larger power lines, power generators, pipelines, and trains was negligible at distances greater than $5 \mathrm{~km}$ from those noise sources. Local lightning, wind, and rainstorms can also degrade data quality. These were avoided by not recording during active thunderstorm periods, burying the magnetic induction coils and keeping the electric dipole wires flat on the ground surface minimizing wind noise.

Predicted values of the electric field can be computed from the measured values of the magnetic field (Vozoff, 1991). The coherence of the predicted electric field with the measured electric field is a measure of the signal-to-noise ratio provided in the multiple coherency plots. Values are normalized between 0 and 1, where values at 0.5 signify signal levels equal to noise levels. For this data set, coherencies were generally at an acceptable level, except at times in the frequency range "dead band" (.1 to $5 \mathrm{HZ}$ ).

The figures in the Appendix represent the field-processed MT data at each station, which includes some data scatter and poor signal-to-noise ratios. Our only effort at removing noisy data points was to visually inspect and select the best signal-tonoise field data to combine into the final data plots.

The impedance polar plots provide a measure of the MT data dimensionality (Reddy and others, 1977). For 1-D resistivity structures, the principal impedance polar diagram (dashed line) is a circle. For 2-D or 3-D resistivity structures, the principal impedance polar diagram (dashed line) elongates either parallel or perpendicular to strike direction. Over resistors, the principal impedance polar diagram elongates perpendicular to strike direction and over conductors, the principal impedance polar diagram elongates parallel to strike direction. Also, for 2-D resistivity structures, the additional impedance polar diagram (solid line) attains the shape of a symmetric clover leaf. For 3-D resistivity structures, the additional impedance polar diagram (solid line) elongates in one direction and its amplitude is comparable to that of the principal impedance polar diagram (dashed line). Stations 1,3, 7, 9, 11, 15, 16, and 23 indicate a 3-D response below $0.3 \mathrm{~Hz}$, while station 24 indicates a 3-D response below $4.8 \mathrm{~Hz}$, and station 25 indicates a 3-D response over all frequencies. 
The tipper can be calculated when the vertical component of the magnetic field is measured. The tipper magnitude is a measure of the tipping of the magnetic field out of the horizontal plane (Vozoff, 1991). The magnitude is zero for the 1-D case and typically increases between 0.1 to 0.5 , and rarely as great as 1, as it responds to vertical and sub-vertical structures. The tipper strike is typically used to help resolve the 90-degree ambiguity in the impedance rotation angle. The tipper magnitude of most of these stations was typically 0.1 to 0.6 over the lower frequencies indicating some vertical structure at depth, with the exception of stations 19 and 21-25 having higher tipper magnitudes indicating larger vertical structure at depth. The HzHx and HzHy coherency is a measure of the signalto-noise ratio of the vertical magnetic field with respect to each of the orthogonal horizontal magnetic field directions. Values are normalized between 0 and 1 , where values at 0.5 signify signal levels equal to noise levels. These threecomponent magnetic field coherencies provide a check on the signal-to-noise ratio of the measured values in the tipper magnitude and tipper strike plots. 


\section{REFERENCES CITED}

Arehart, G.B., Foland, K.A., Naeser, C.W., and Kesler, S.E., 1993, 40Ar/39Ar, K/Ar, and fission track geochronology of sediment-hosted disseminated gold deposits at Post-Betze, Carlin Trend, northeastern Nevada: Economic Geology, vol. 88 , no. 3, p. $622-646$.

Bendat, J.S., and Piersol, A.G., 1971, Random Data: Analysis and Measurement Procedures: New York, Wiley Interscience, $407 \mathrm{p}$.

Dobrin, M.D., and Savit, C.H., 1988, Introduction to Geophysical Prospecting (4th ed.): New York, McGraw-Hill, $867 p$.

Eberhart-Phillips, D., Stanley, W.D., Rodriguez, B.D. and Lutter, W.J., 1995, Surface seismic and electrical methods to detect fluids related to faulting: Journal of Geophysical Research, vol. 100, no. B7, p. 12,919-12,936.

EMI, 1996, MT-1 magnetotelluric system operation manual, version 3.2: ElectroMagnetic Instruments, Inc., Richmond, California, $220 \mathrm{p}$.

Gamble, T.D., Goubau, W.M. and Clarke, J., 1979, Error analysis for remote reference magnetotellurics: Geophysics, v. 44, no. 5, p. 959-968.

Grauch, V.J.S., 2003, Personal communications: U.S. Geological Survey, Denver, Colorado.

Ilchik, R.P. and Barton, M.D., 1997, An amagmatic origin of Carlin-type gold deposits: Economic Geology, vol. 92, no. 3, p. 269-288.

Keller, G.V., 1987, Rock and mineral properties, in Electromagnetic Methods in Applied Geophysics Theory: M.N. Nabighian, Ed., Society of Exploration Geophysicists, Tulsa, Oklahoma, v. 1, p. 1351 .

Keller, G.V., 1989, Electrical properties, in Carmichael, R.S., Ed., Practical handbook of physical properties of rocks and minerals: CRC Press, Boca Raton, Florida, p. 359-427.

Nelson, P.H. and Anderson, L.A., 1992, Physical properties of ash flow tuff from Yucca Mountain, Nevada: Journal of Geophysical Research, vol. 97, no. B5, p. 6823-6841. 
Palacky, G.J., 1987, Resistivity characteristics of geologic targets, in Electromagnetic Methods in Applied Geophysics Theory: M.N. Nabighian, Ed., Society of Exploration Geophysicists, Tulsa, Oklahoma, vol. 1, p. 53129.

Radtke, A.S., 1985, Geology of the Carlin gold deposit, Nevada: U.S. Geological Survey Professional Paper 1267, 124 p.

Reddy, I.K., Rankin, D., and Phillips, R.J., 1977, Threedimensional modelling in magnetotelluric and magnetic variational sounding: Geophysics Journal of the Royal Astronomical Society, vol. 51, p. 313-325.

Shawe, D.R., 1991, Structurally controlled gold trends imply large gold resources in Nevada, in Geology and ore deposits of the Great Basin, Symposium Proceedings: Raines, G.L., Lisle, R.E., Schafe, R.W., Wilkinson, W.H., Eds., Geological Society of Nevada, Reno, vol. 1, p. 199-212.

Sillitoe, R.H. and Bonham, H.F., 1990, Sediment-hosted gold deposits; distal products of magmatic-hydrothermal systems: Geology, vol. 18, no. 2, p. 157-161.

Sternberg, B.K., Washburne, J.C., and Pellerin, L., 1988, Correction for the static shift in magnetotellurics using transient electromagnetic soundings: Geophysics, vol. 53, p. 1459-1468.

Tosdal, R.M., 1998, Contributions to the gold metallogeny of northern Nevada: U.S. Geological Survey Open-File Report 98$338,290 \mathrm{p}$.

Vozoff, K., 1972, The magnetotelluric method in the exploration of sedimentary basins: Geophysics, vol. 37, p. 98-141.

Vozoff, K., 1991, The magnetotelluric method, in Electromagnetic methods in applied geophysics: M.N. Nabighian, Ed., Society of Exploration Geophysicists, Tulsa, Oklahoma, vol. 2, part B, p. 641-711. 


\section{APPENDIX MAGNETOTELLURIC DATA PLOTS}

There are eight separate plots for each station:

1. Apparent Resistivity for the rotated maximum ( $x$ symbol) and minimum (o symbol) modes

2. Impedance Phase for the rotated maximum ( $x$ symbol) and minimum (o symbol) modes

3. Impedance Skew for the impedance tensor

4. Multiple Coherency for the rotated maximum ( $x$ symbol) and minimum (o symbol) modes of the electric field

5. Impedance Polar Plots (at 12 selected frequencies), for principal impedence (dashed line) and additional impedance (solid lines)

6. Tipper Magnitude for the vertical magnetic field

7. Tipper Strike for the vertical magnetic field

8. $\mathrm{HzHx}$ ( $x$ symbol) and HzHy (o symbol) Coherency

Refer to the "Magnetotelluric Data" section in this report for an explanation of these plots. 


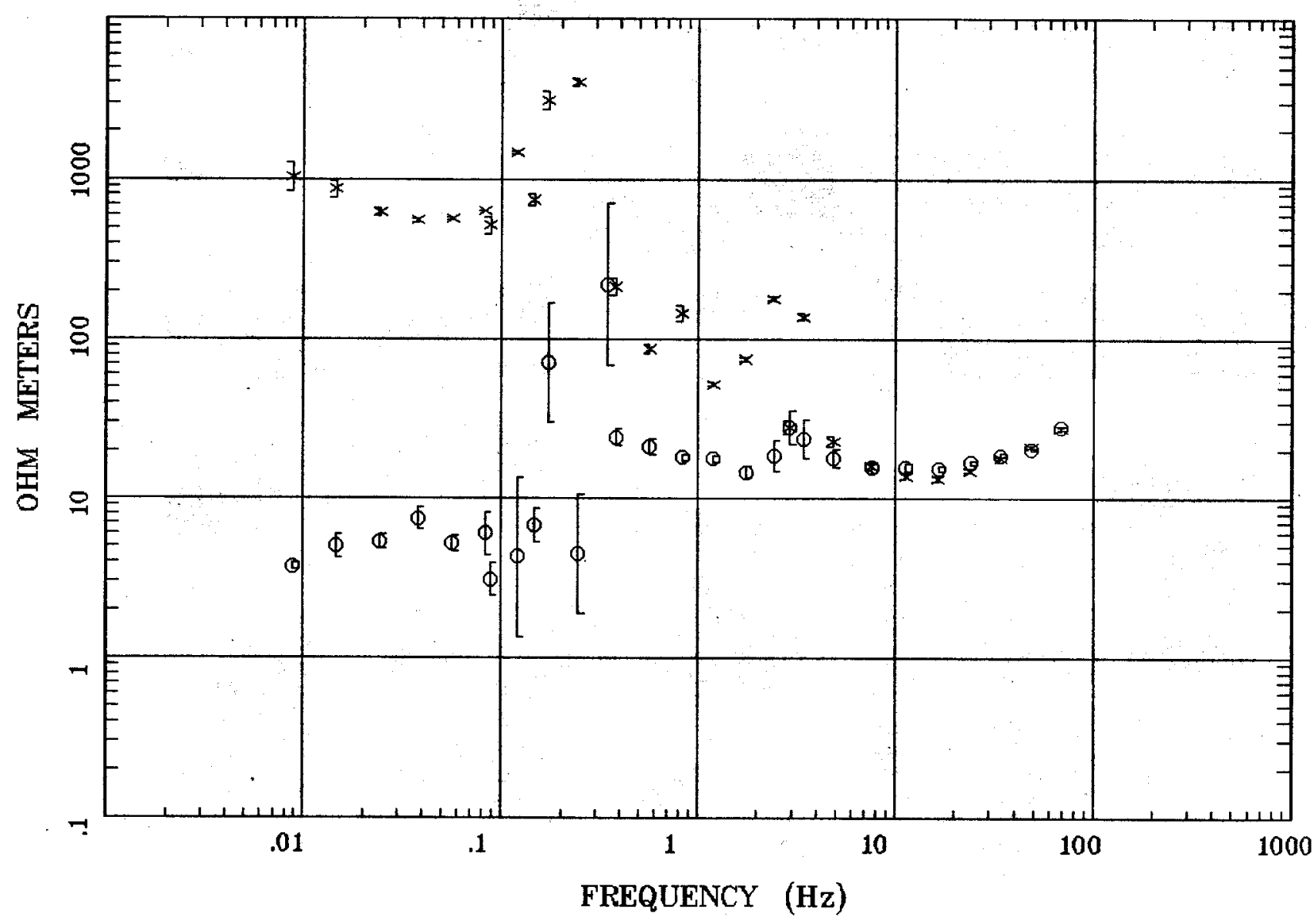

Client:

Remote: none Acquired: 15:5 Sep 04, 2003 Survey Co:USGS
Rotation:

Filename: ap01m.avg

Channels: Ch1 Ch2 Ch3 Ch4 Ch5 Ch1 Ch2 Plotted: 14:17 Sep 23, 2003

< EMI - ElectroMagnetic Instruments > 


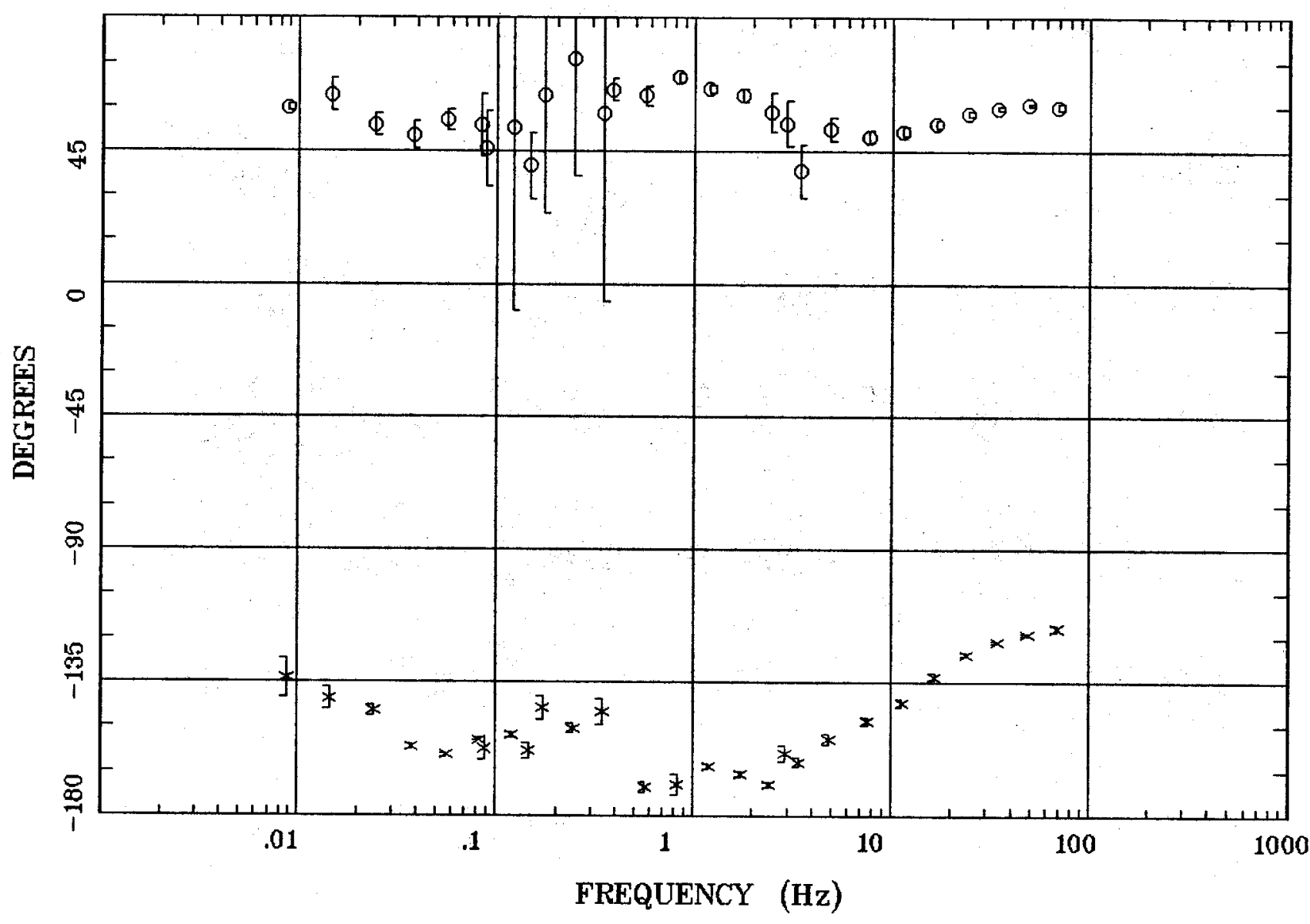

Client:

Remote: none Acquired: 15:5 Sep 04, 2003 Survey Co:USGS
Rotation:

Filename: ap01m.avg

Channels: Ch1 Ch2 Ch3 Ch4 Ch5 Ch1 Ch2

Plotted: 14:18 Sep 23, 2003

\& EMI - ElectroMagnetic Instruments 


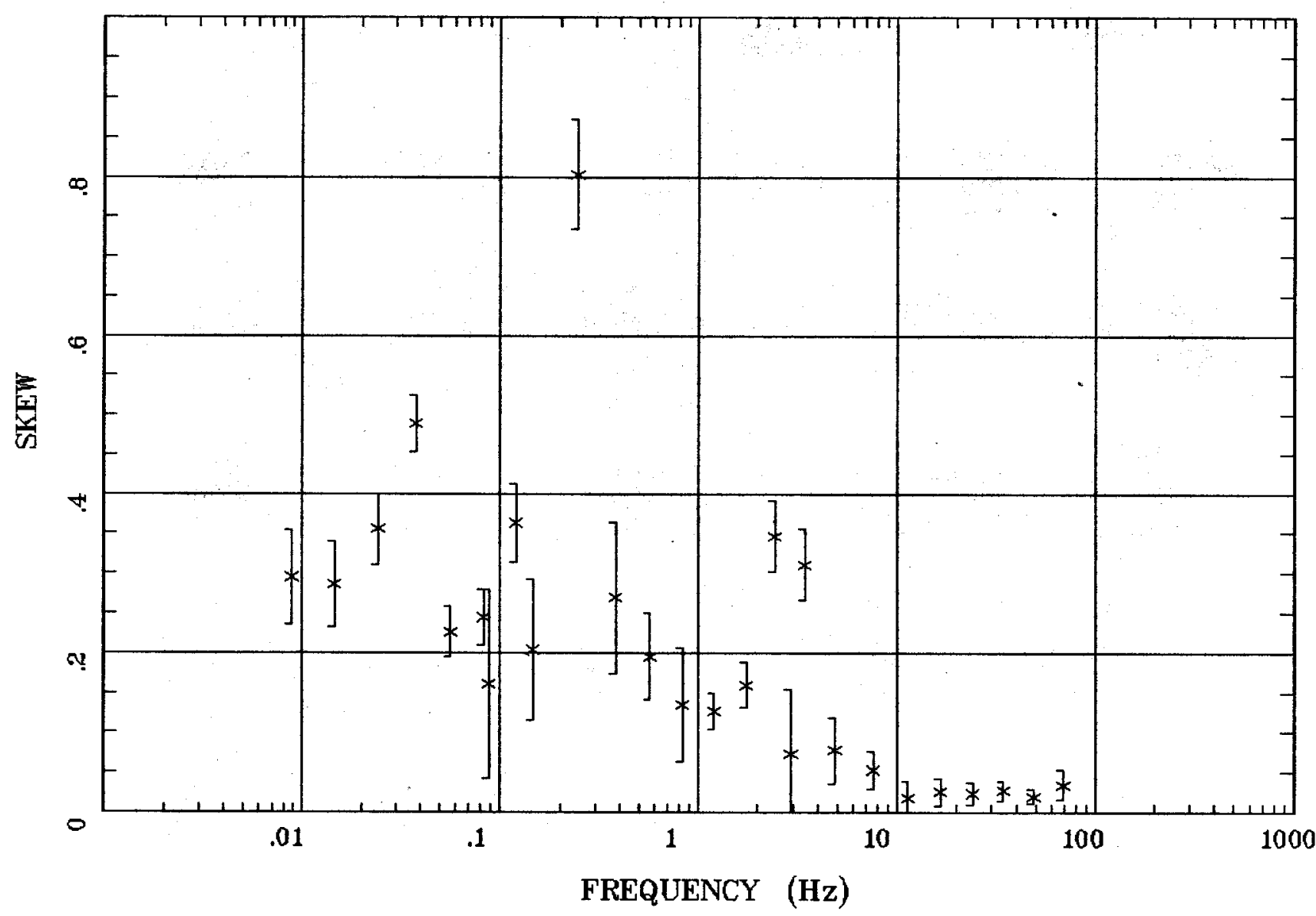

Client:

Remote: none

Acquired: 15:5 Sep 04, 2003 Survey Co:USGS
Rotation:

Filename: ap01m.avg

Channels: Ch1 Ch2 Ch3 Ch4 Ch5 Ch1 Ch2

Plotted: 14:19 Sep 23, 2003

< EMI - ElectroMagnetic Instruments 


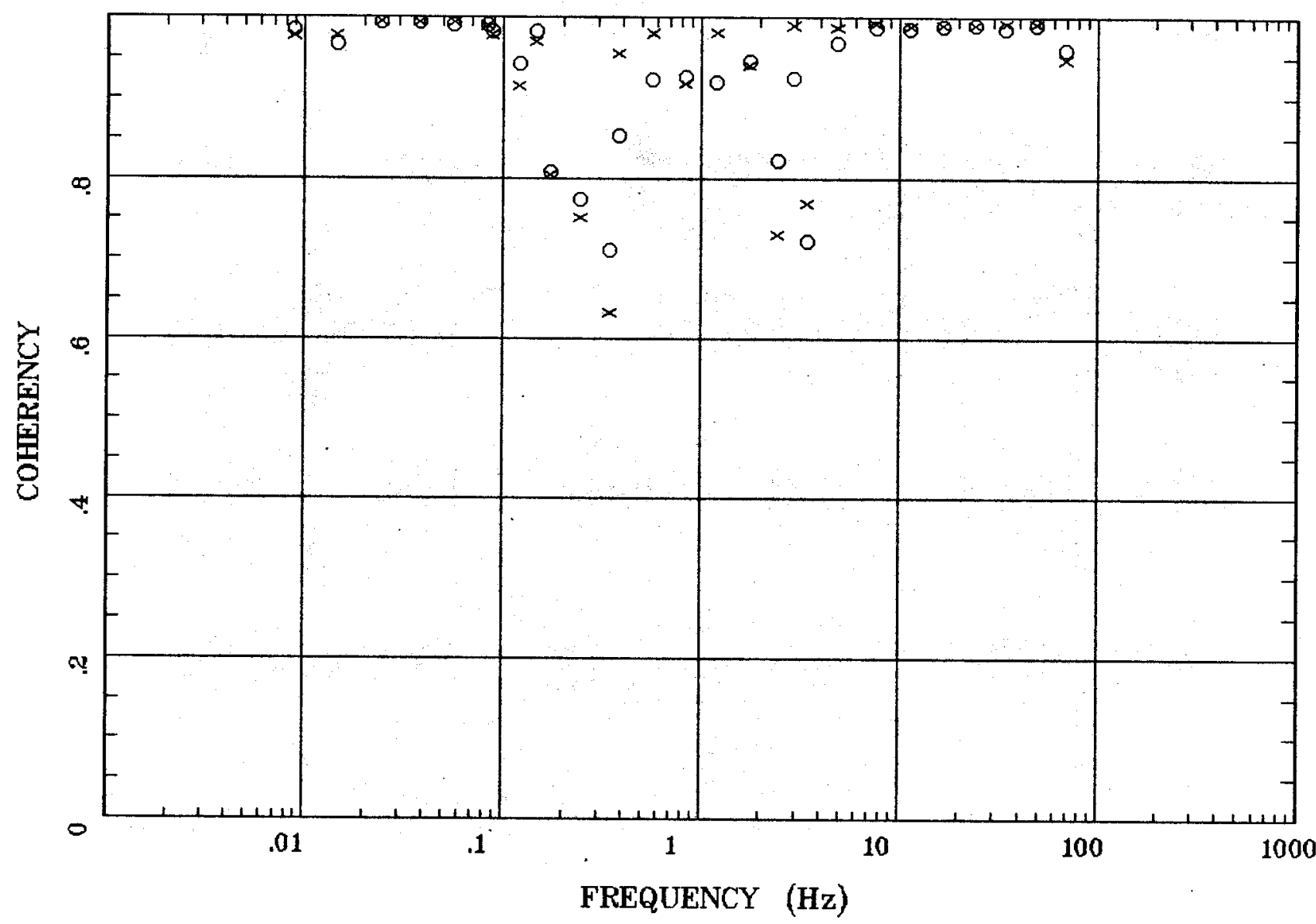

Client:

Remote: none

Acquired: 15:5 Sep 04, 2003

Survey Co:USGS
Rotation:

Filename: ap01m.avg

Channels: Ch1 Ch2 Ch3 Ch4 Ch5 Ch1 Ch2

Plotted: 14:18 Sep 23, 2003

$<$ EMI - ElectroMagnetic Instruments 


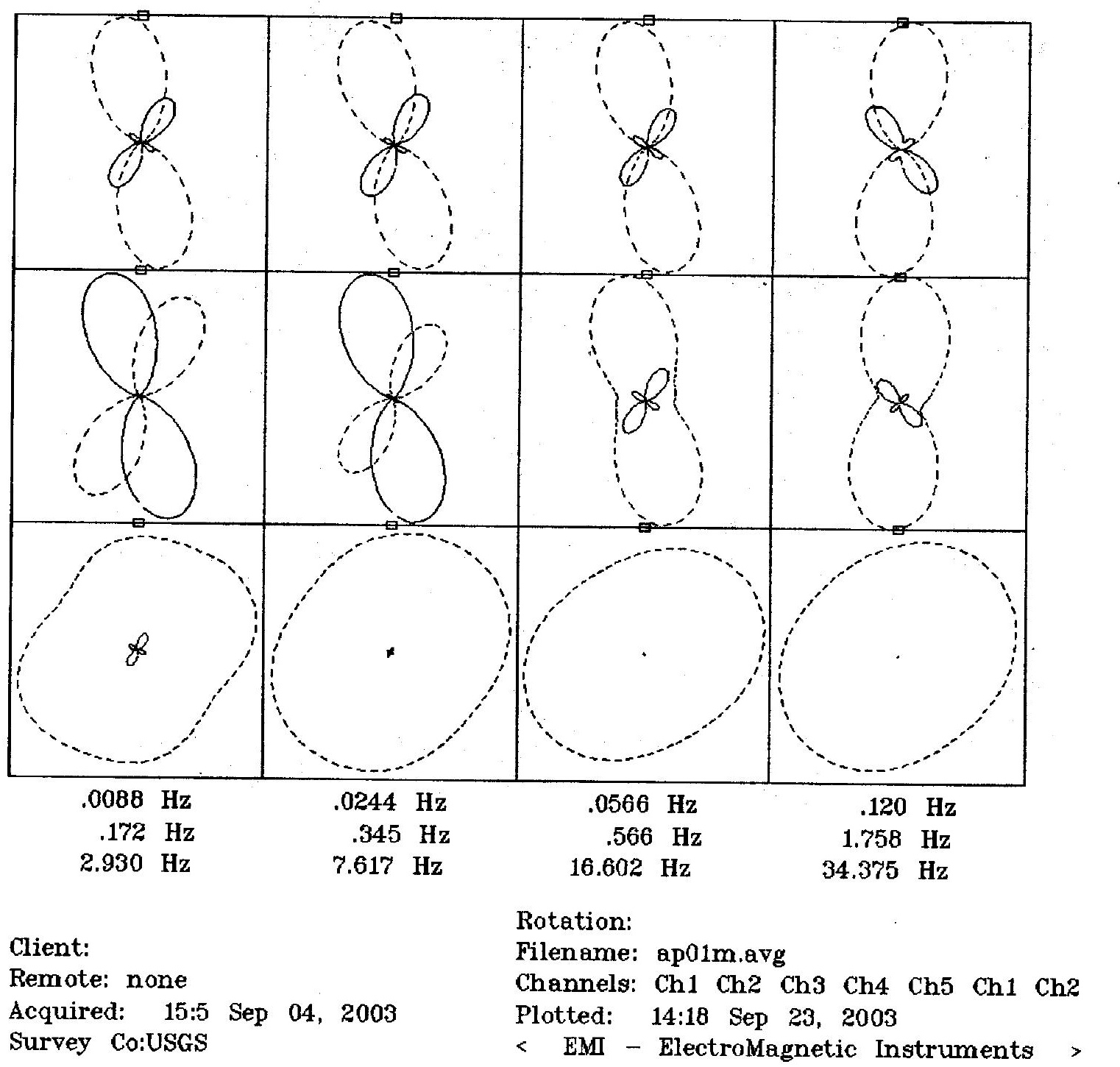




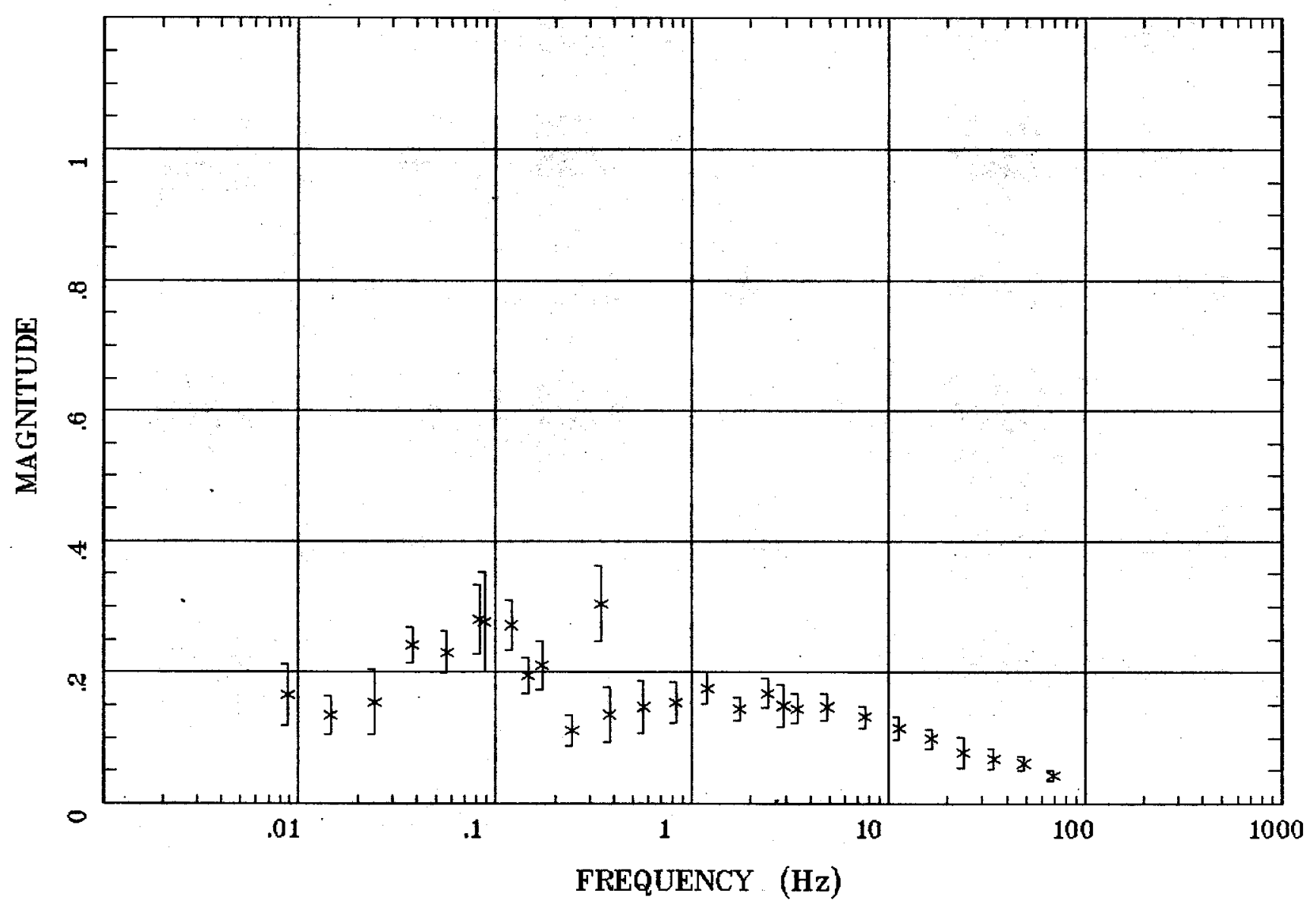

Client:

Remote: none

Acquired: 15:5 Sep 04, 2003 Survey Co:USGS
Rotation:

Filename: ap01m.avg

Channels: Ch1 Ch2 Ch3 Ch4 Ch5 Ch1 Ch2

Plotted: 14:18 Sep 23, 2003

< EMI - ElectroMagnetic Instruments > 


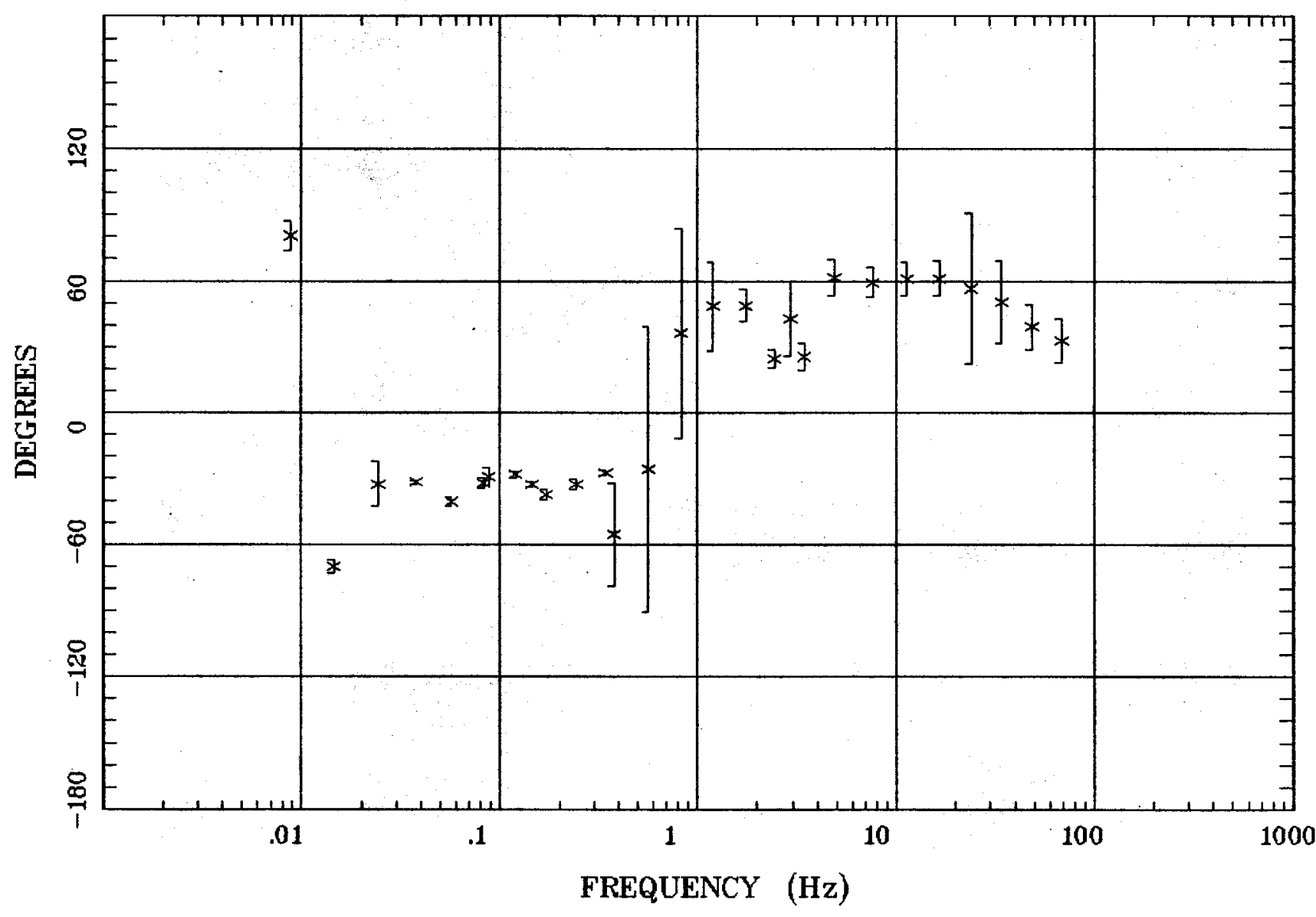

Client:

Remote: none

Acquired: 15:5 Sep 04, 2003 Survey Co:USGS
Rotation:

Filename: ap01m.avg

Channels: Ch1 Ch2 Ch3 Ch4 Ch5 Ch1 Ch2

Plotted: $14: 18$ Sep 23, 2003

< EMI - ElectroMagnetic Instruments 


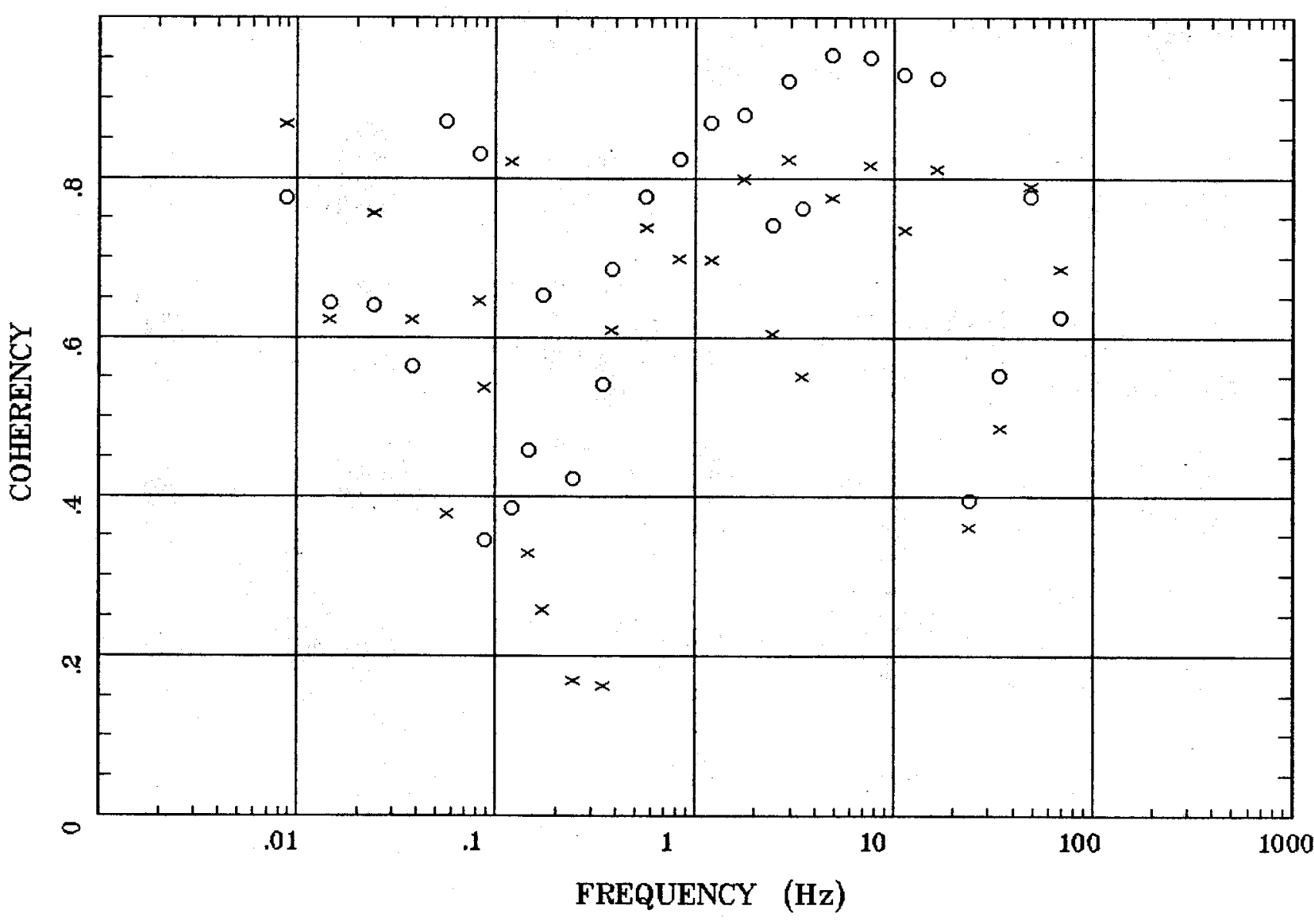

Client:

Remote: none Acquired: 15:5 Sep 04, 2003 Survey Co:USGS
Rotation:

Filename: ap01m.avg

Channels: Ch1 Ch2 Ch3 Ch4 Ch5 Ch1 Ch2 Plotted: 14:18 Sep 23, 2003

$<$ EMI - ElectroMagnetic Instruments 


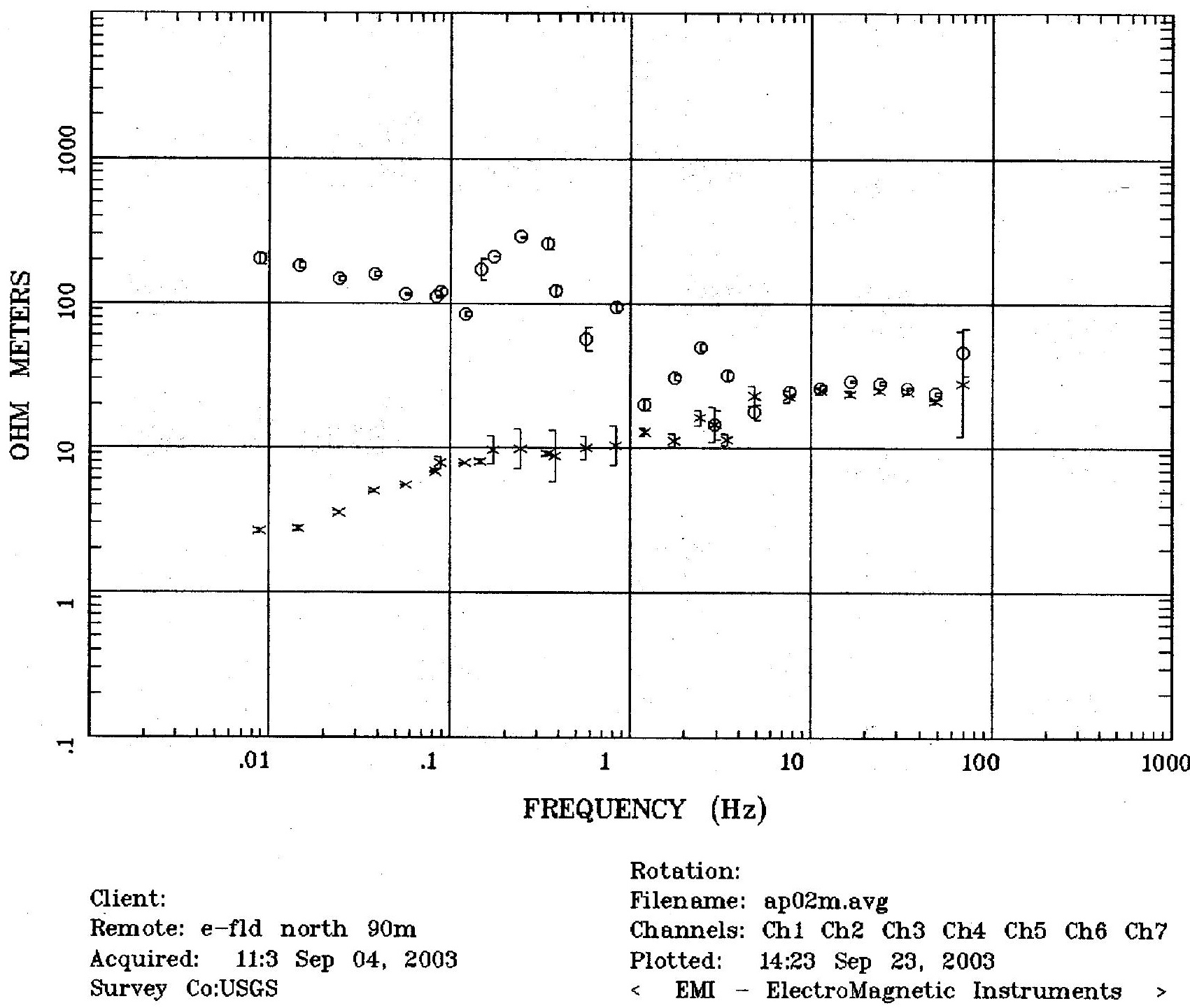




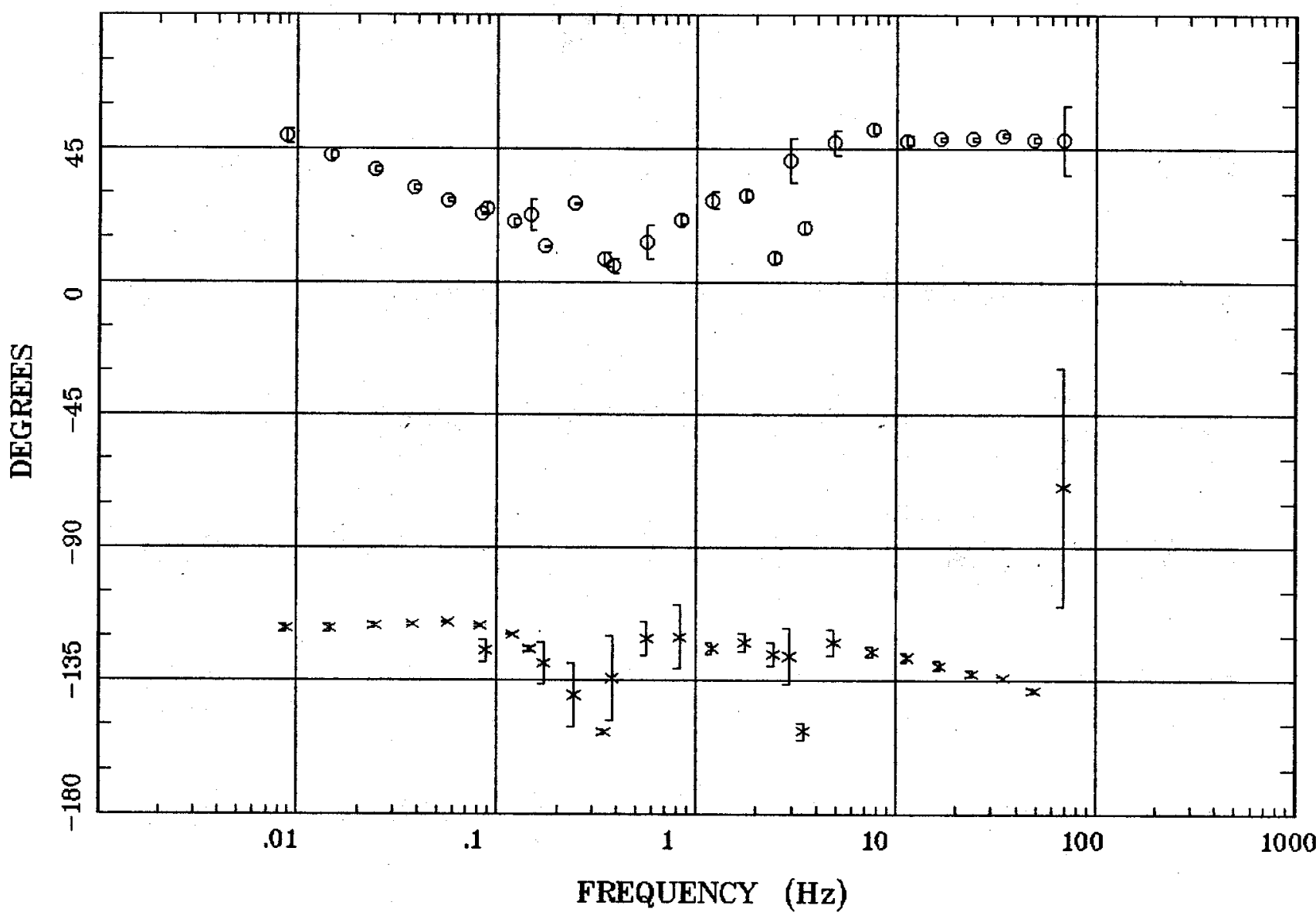

Client:

Remote: e-fld north $90 \mathrm{~m}$ Acquired: 11:3 Sep 04, 2003 Survey Co:USGS
Rotation:

Filename: ap02m.avg

Channels: Ch1 Ch2 Ch3 ch4 Ch5 Ch6 Ch7 Plotted: 14:23 Sep 23, 2003

$<$ EMI - ElectroMagnetic Instruments 


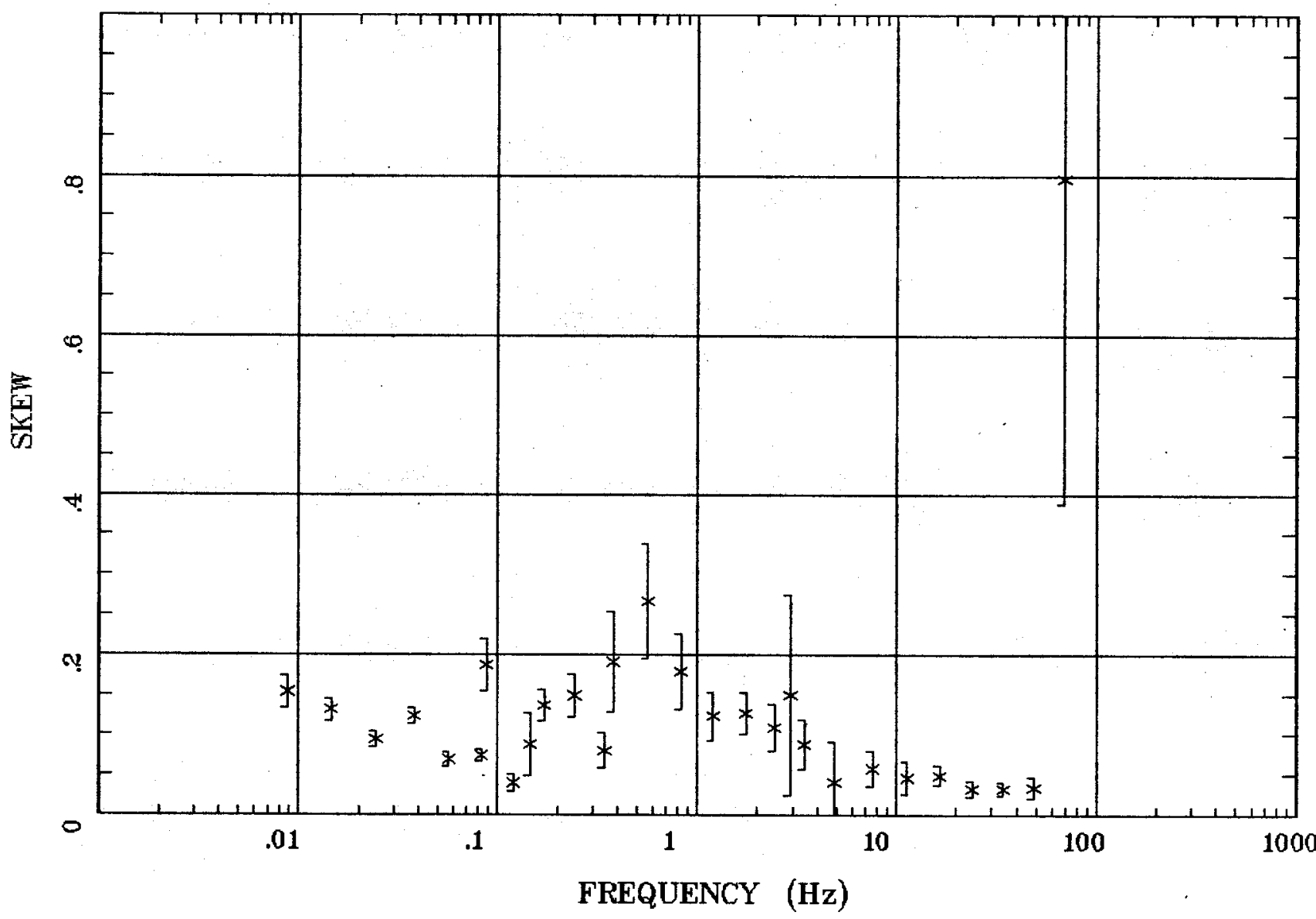

Client:

Remote: e-fld north $90 \mathrm{~m}$ Acquired: 11:3 Sep 04, 2003 Survey Co:USGS
Rotation:

Filename: ap02m.avg

Channels: Ch1 Ch2 Ch3 Ch4 Ch5 Ch6 Ch7 Plotted: 14:23 Sep 23, 2003

< EMI - ElectroMagnetic Instruments 
E MULT Coh.

Wells, NV 100kmap

Station 2

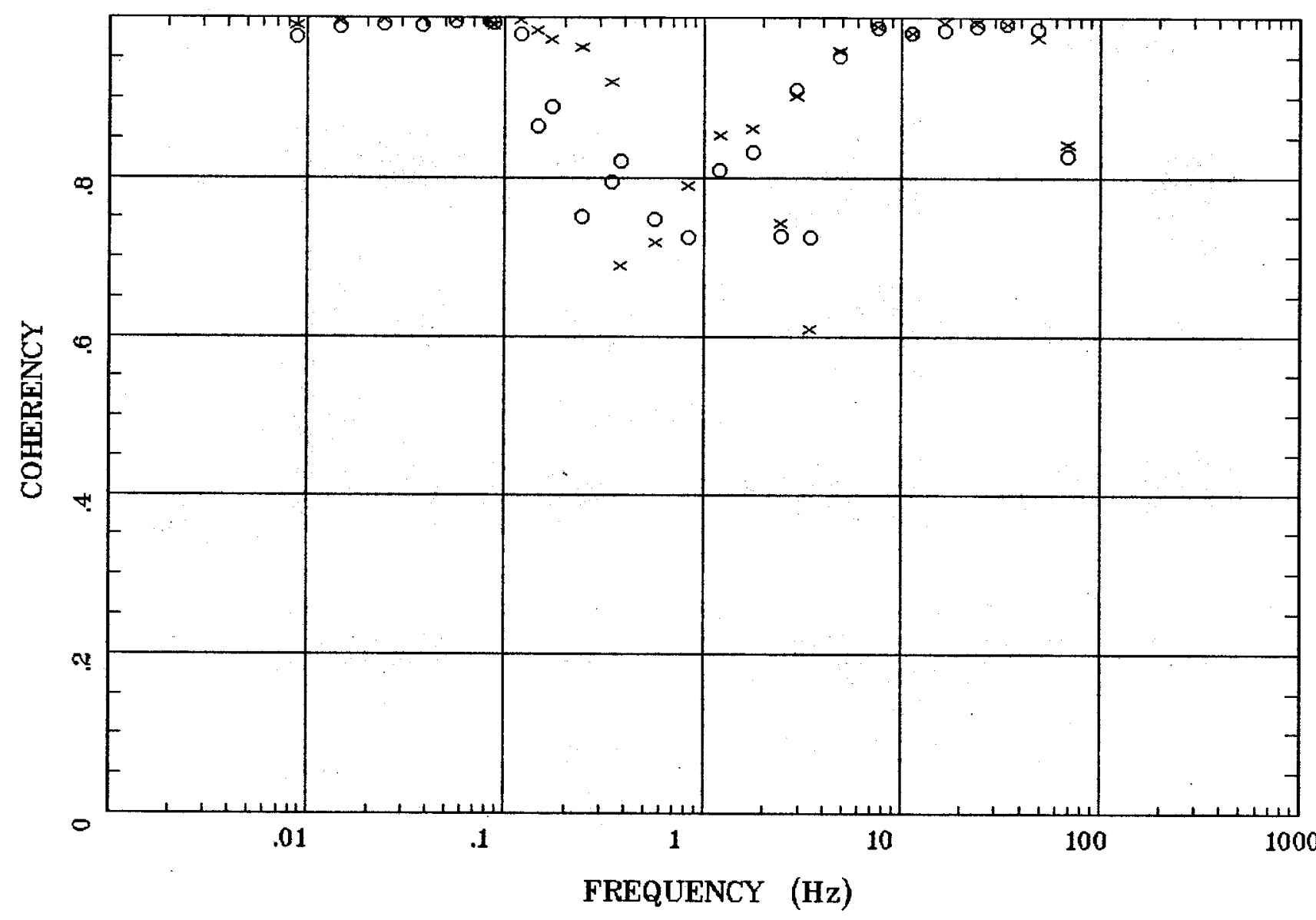

Client:

Remote: e-fld north $90 \mathrm{~m}$ Acquired: 11:3 Sep 04, 2003 Survey Co:USGS
Rotation:

Filename: ap02m.avg

Channels: Ch1 Ch2 Ch3 Ch4 Ch5 Ch6 Ch7 Plotted: 14:23 Sep 23, 2003

$<$ EMI - ElectroMagnetic Instruments 


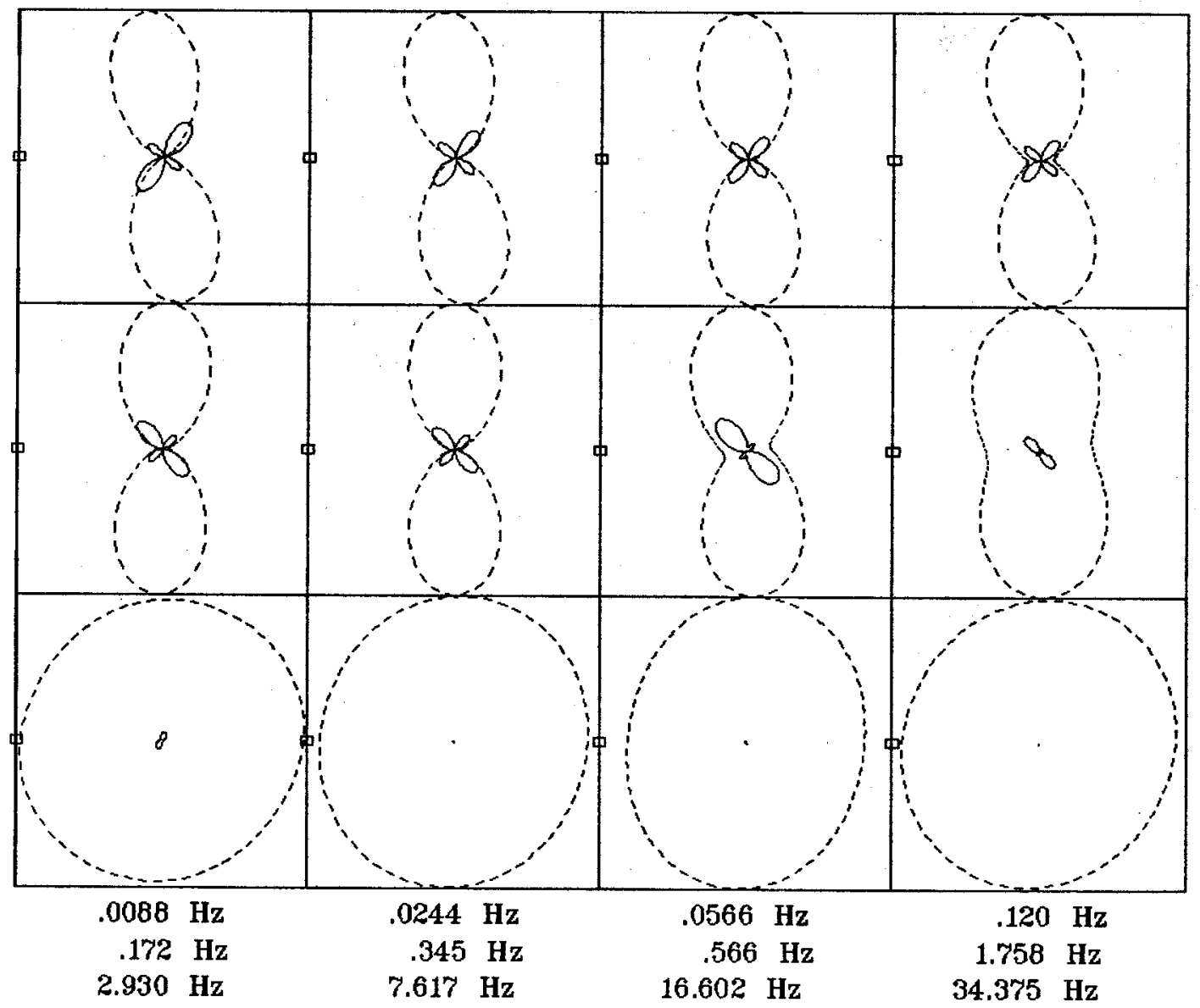

Client:

Remote: e-fld north $90 \mathrm{~m}$ Acquired: 11:3 Sep 04, 2003 Survey Co:USGS
Rotation:

Filename: ap02m.avg

Channels: Ch1 Ch2 Ch3 Ch4 Ch5 Ch6 Ch7 Plotted: 14:23 Sep 23, 2003

< EMI - ElectroMagnetic Instruments > 


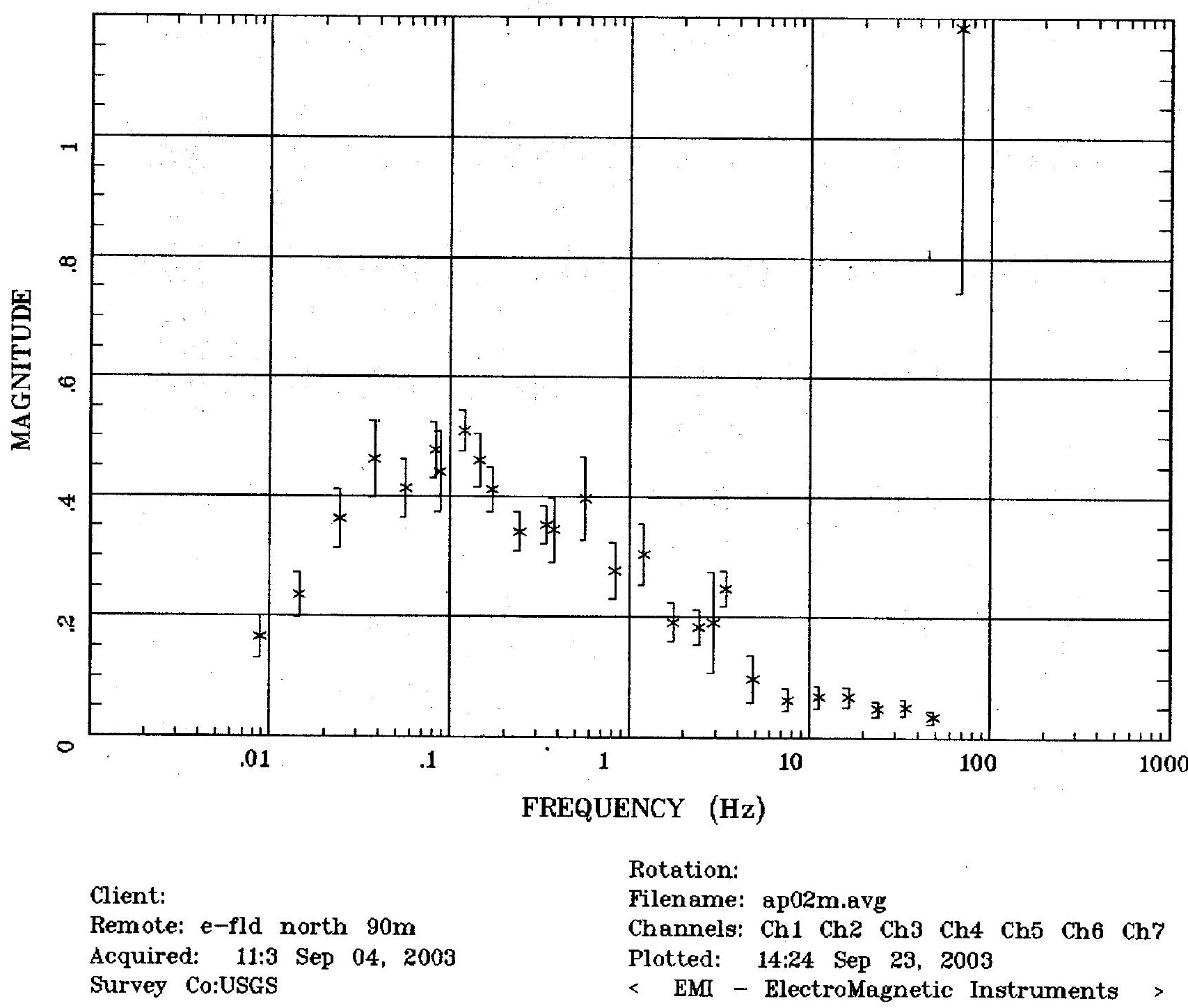




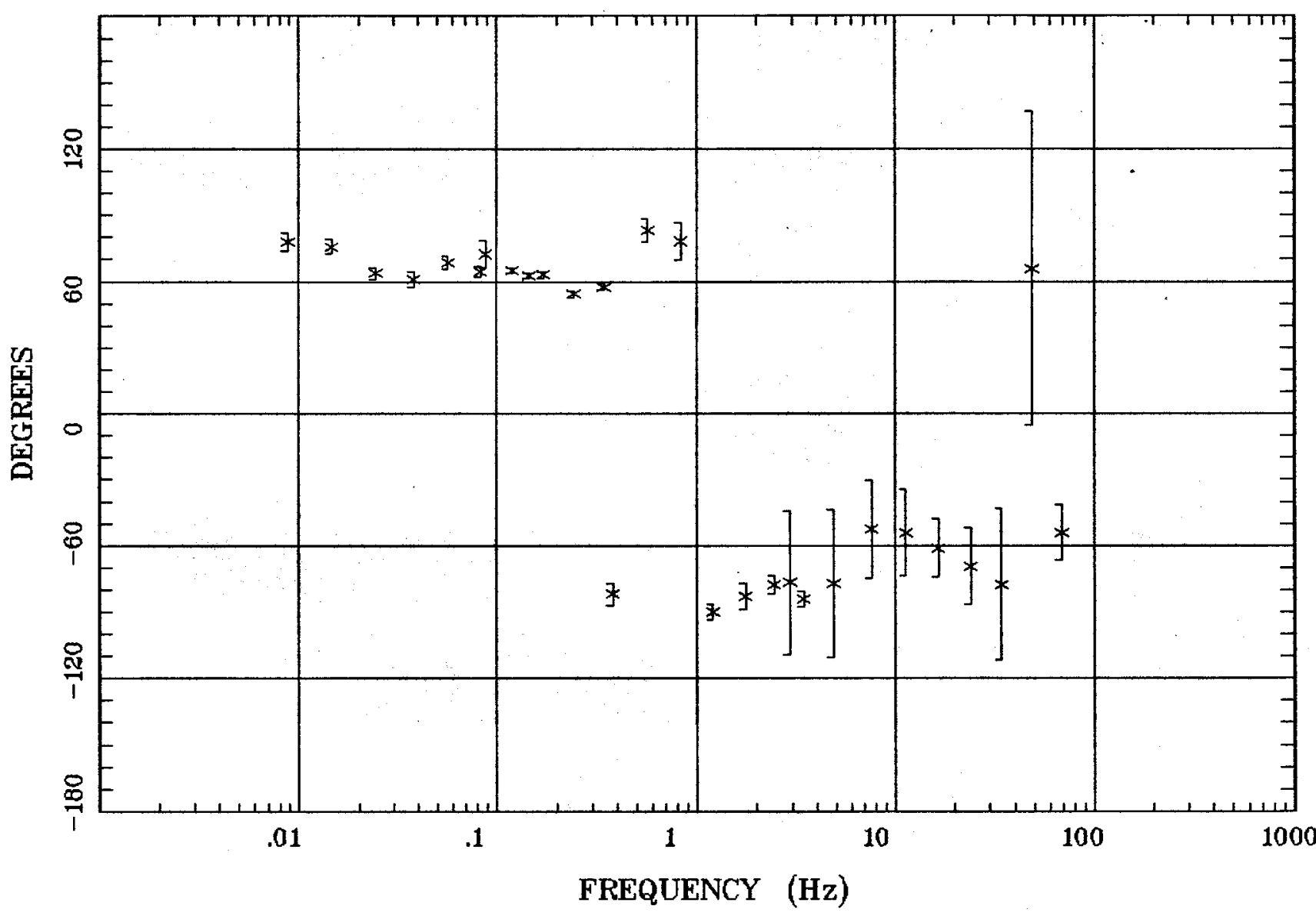

Client:

Remote: e-fld north $90 \mathrm{~m}$

Acquired: 11:3 Sep 04, 2003 Survey Co:USGS
Rotation:

Filename: ap02m.avg

Channels: Ch1 Ch2 Ch3 Ch4 Ch5 Ch6 Chr Plotted: 14:24 Sep 23, 2003

$<$ EMI - ElectroMagnetic Instruments 
HzHx.x Coh HzHy.o

Wells, NV 100kmap

Station 2

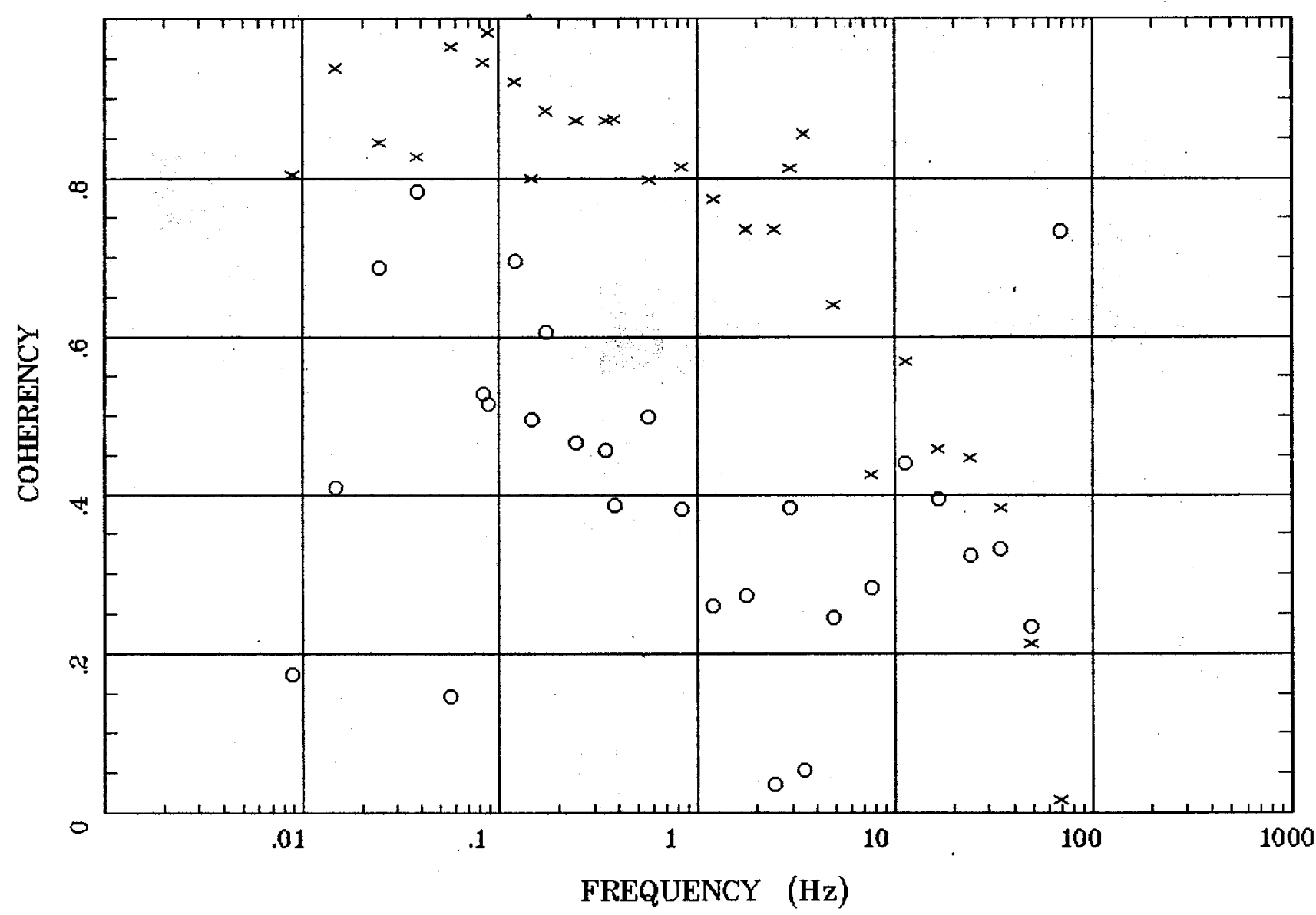

Client:

Remote: e-fld north $90 \mathrm{~m}$ Acquired: 11:3 Sep 04, 2003 Survey Co:USGS
Rotation:

Filename: ap02m.avg

Channels: Ch1 Ch2 Ch3 Ch4 Ch5 Ch6 Ch7 Plotted: 14:24 Sep 23, 2003

$<$ EMI - ElectroMagnetic Instruments 


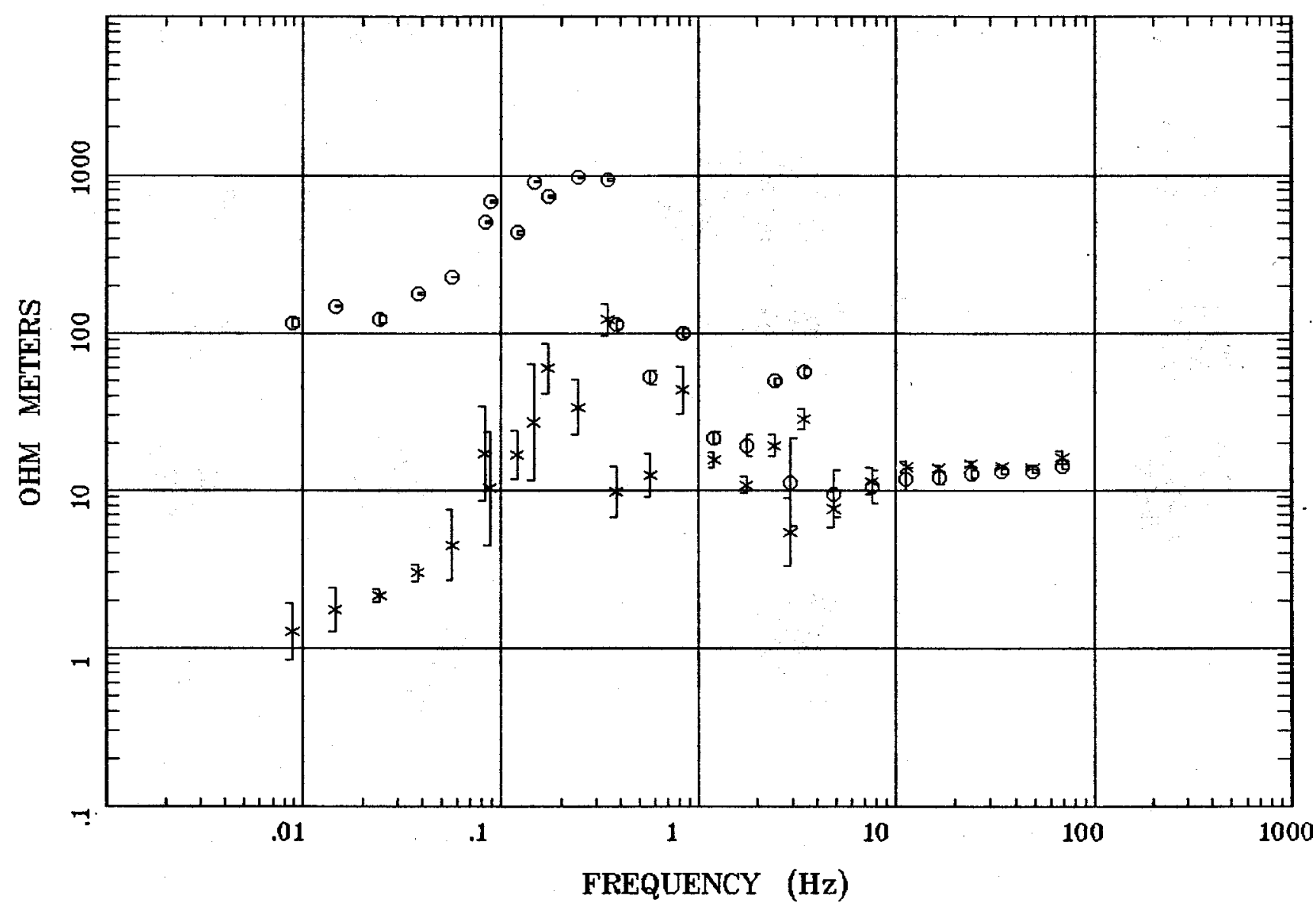

Client:

Remote: e-fld south $90 \mathrm{~m}$ Acquired: 10:1 Sep 05, 2003 Survey Co:USGS
Rotation:

Filename: ap03m.avg

Channels: Ch1 Ch2 Ch3 Ch4 Ch5 Ch6 Ch7 Plotted: 15:08 Sep 23, 2003

$<$ EMI - ElectroMagnetic Instruments > 
Wendover, NV 100kmap Station 3

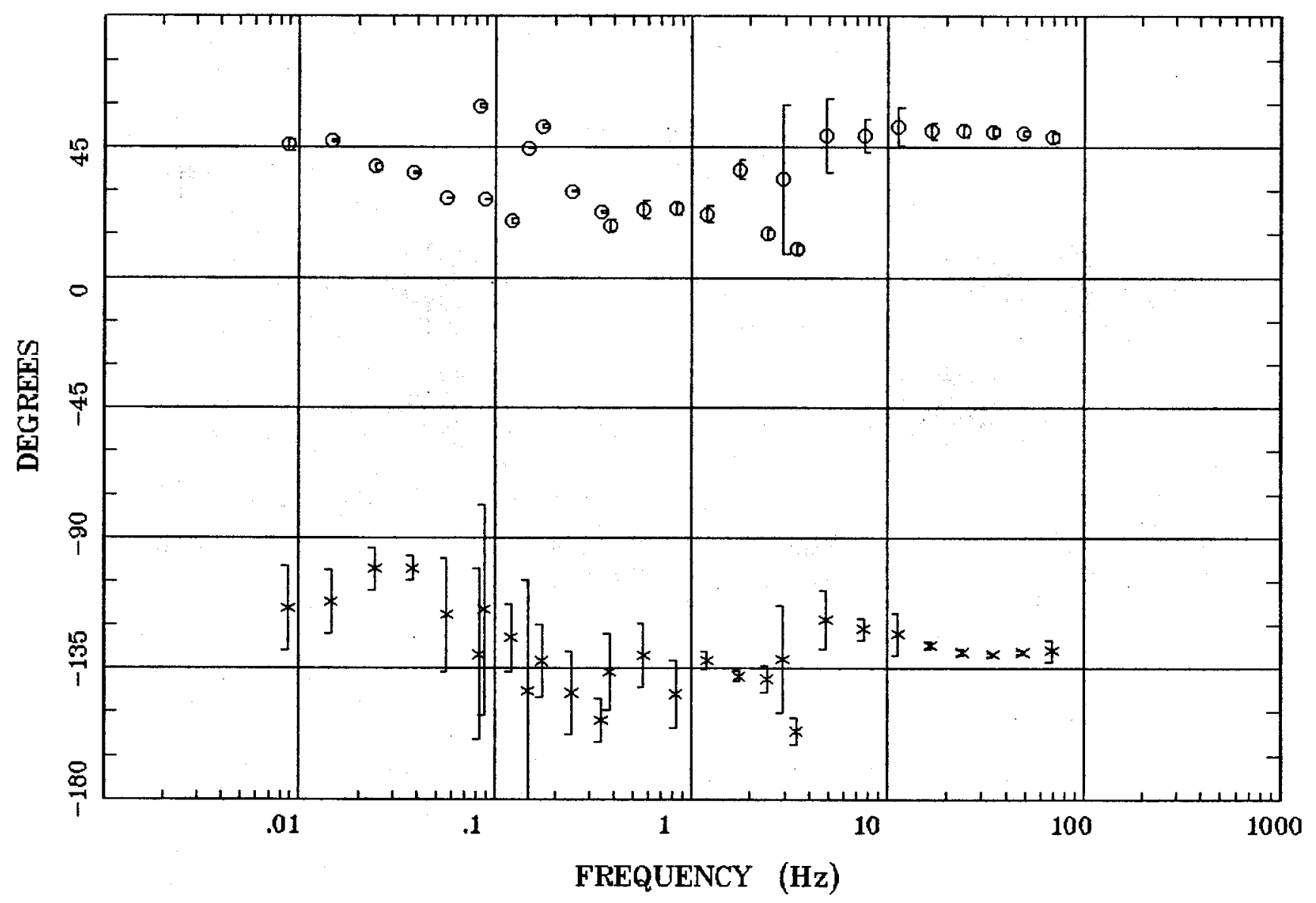

Client:

Remote: e-fld south $90 \mathrm{~m}$

Acquired: 10:1 Sep 05, 2003

Survey Co:USGS
Rotation:

Filename: ap03m.avg

Channels: Ch1 Ch2 Ch3 Ch4 Ch5 Ch6 Ch7

Plotted: 15:08 Sep 23, 2003

$<$ EMI - ElectroMagnetic Instruments 


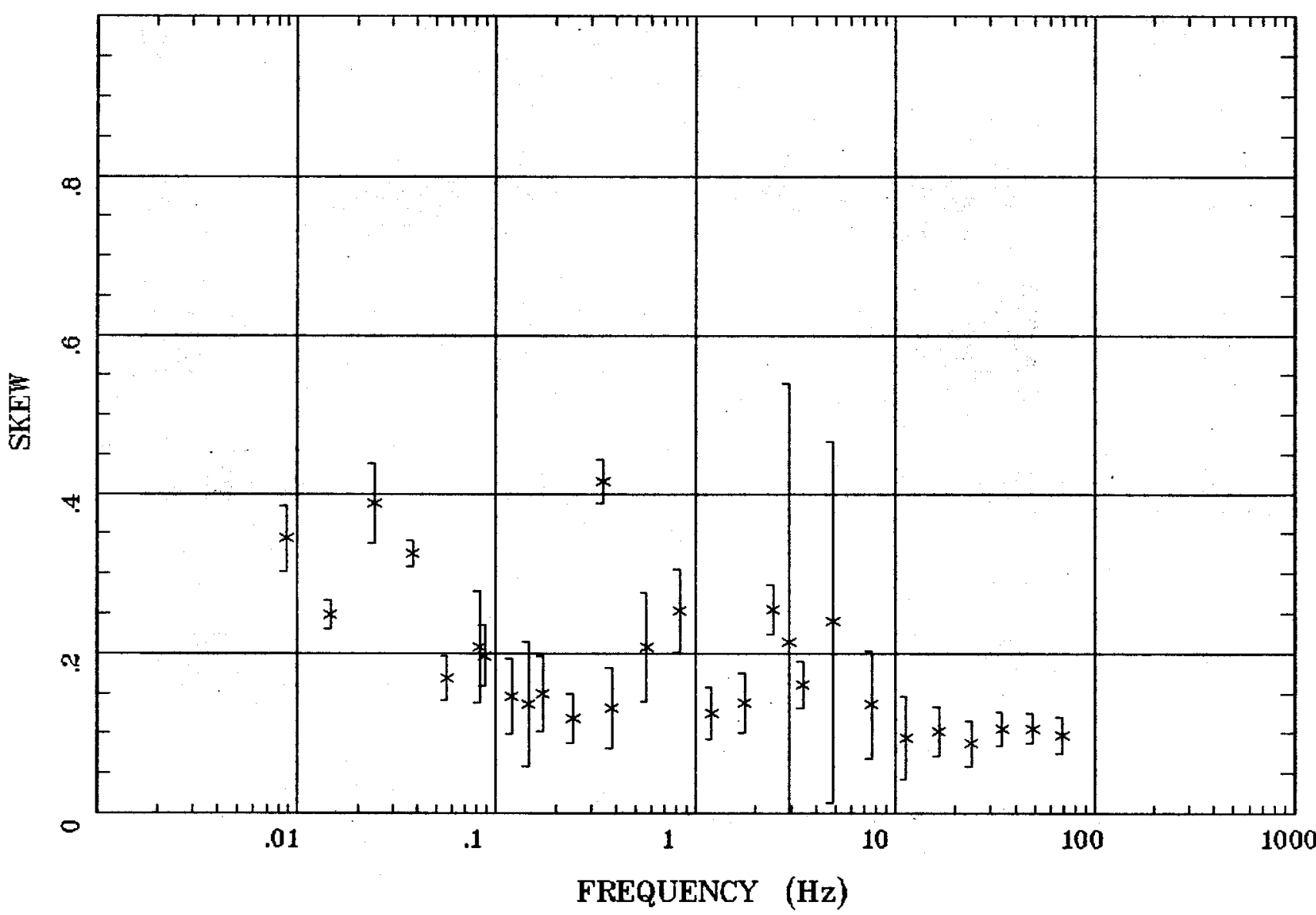

Client:

Remote: e-fld south $90 \mathrm{~m}$ Acquired: 10:1 Sep 05, 2003 Survey Co:USGS
Rotation:

Filename: ap03m.avg

Channels: Ch1 Ch2 Ch3 Ch4 Ch5 Ch6 Ch7 Platted: 15:08 Sep 23, 2003

< EMI - ElectroMagnetic Instruments 


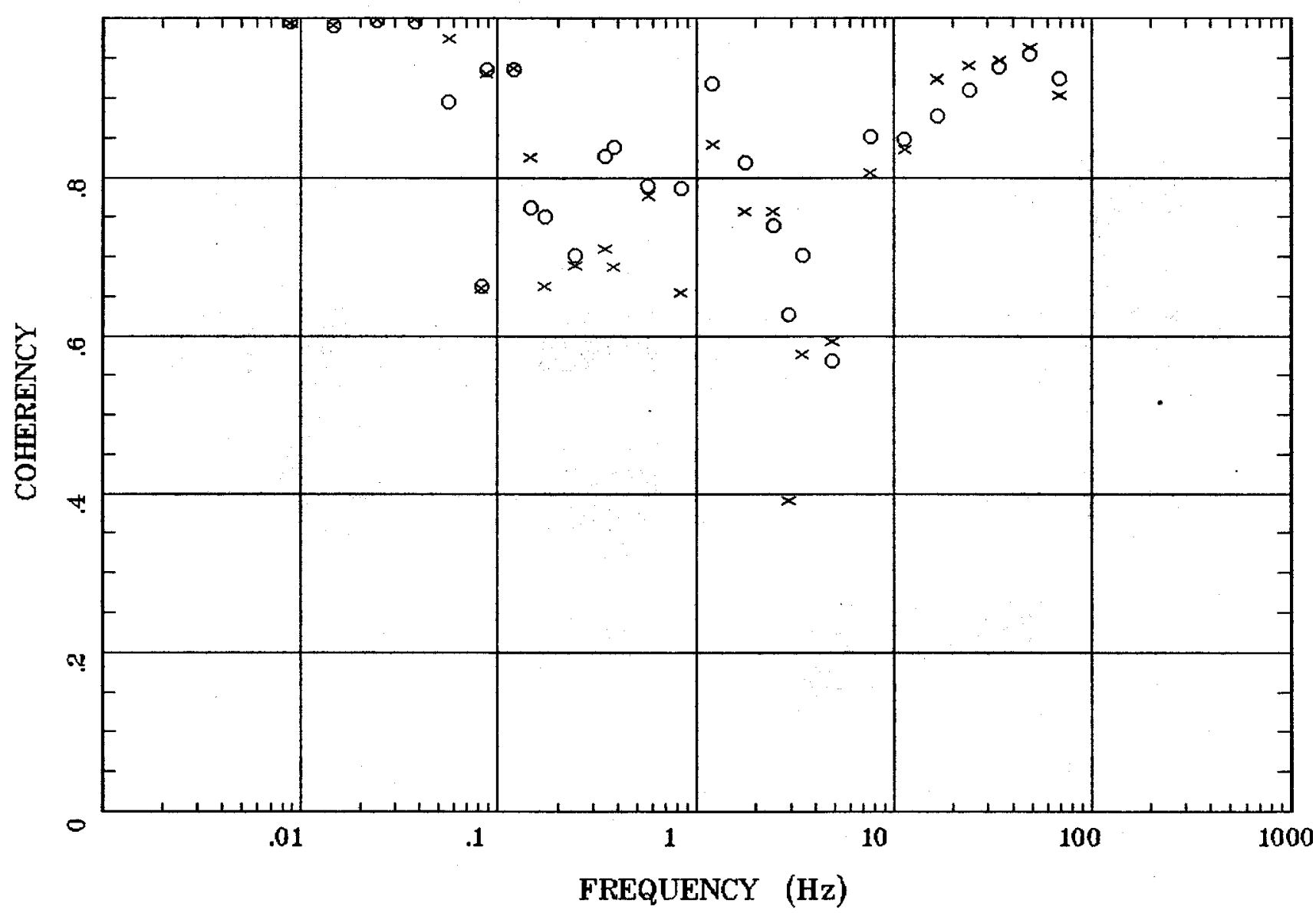

Client:

Remote: e-fld south $90 \mathrm{~m}$ Acquired: 10:1 Sep 05, 2003 Survey Co:USGS
Rotation:

Filename: ap03m.avg

Channels: Ch1 Ch2 Ch3 ch4 ch5 Ch6 chr Plotted: 15:08 Sep 23, 2003

< EMI - ElectroMagnetic Instruments 


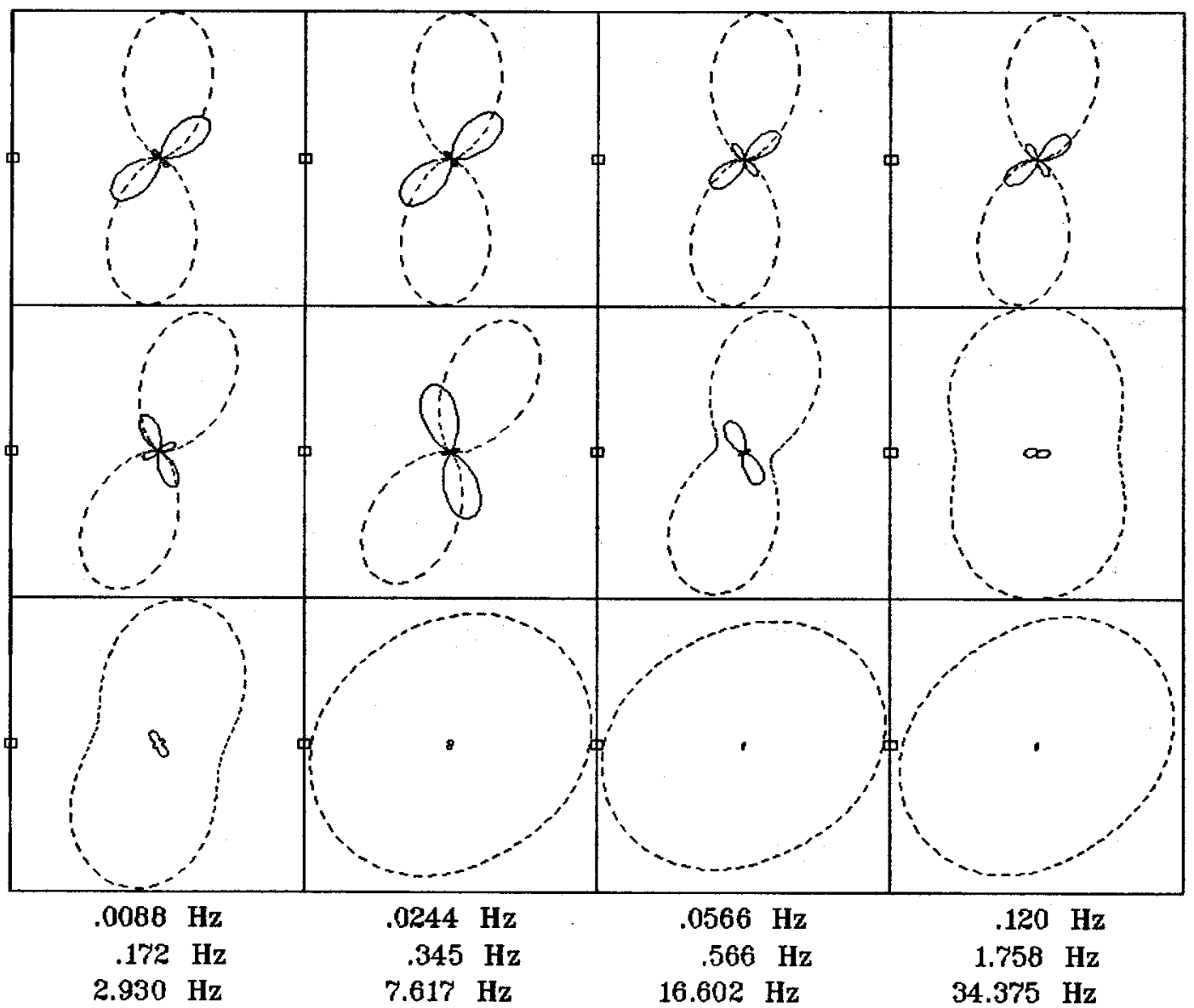

Client:

Remote: e-fld south $90 \mathrm{~m}$ Acquired: 10:1 Sep 05, 2003 Survey Co:USGS

\section{Rotation:}

Filename: ap03m.avg

Channels: Ch1 Ch2 Ch3 Ch4 Ch5 Ch6 Ch7 Plotted: 15:08 Sep 23, 2003

< EMI - ElectroMagnetic Instruments > 


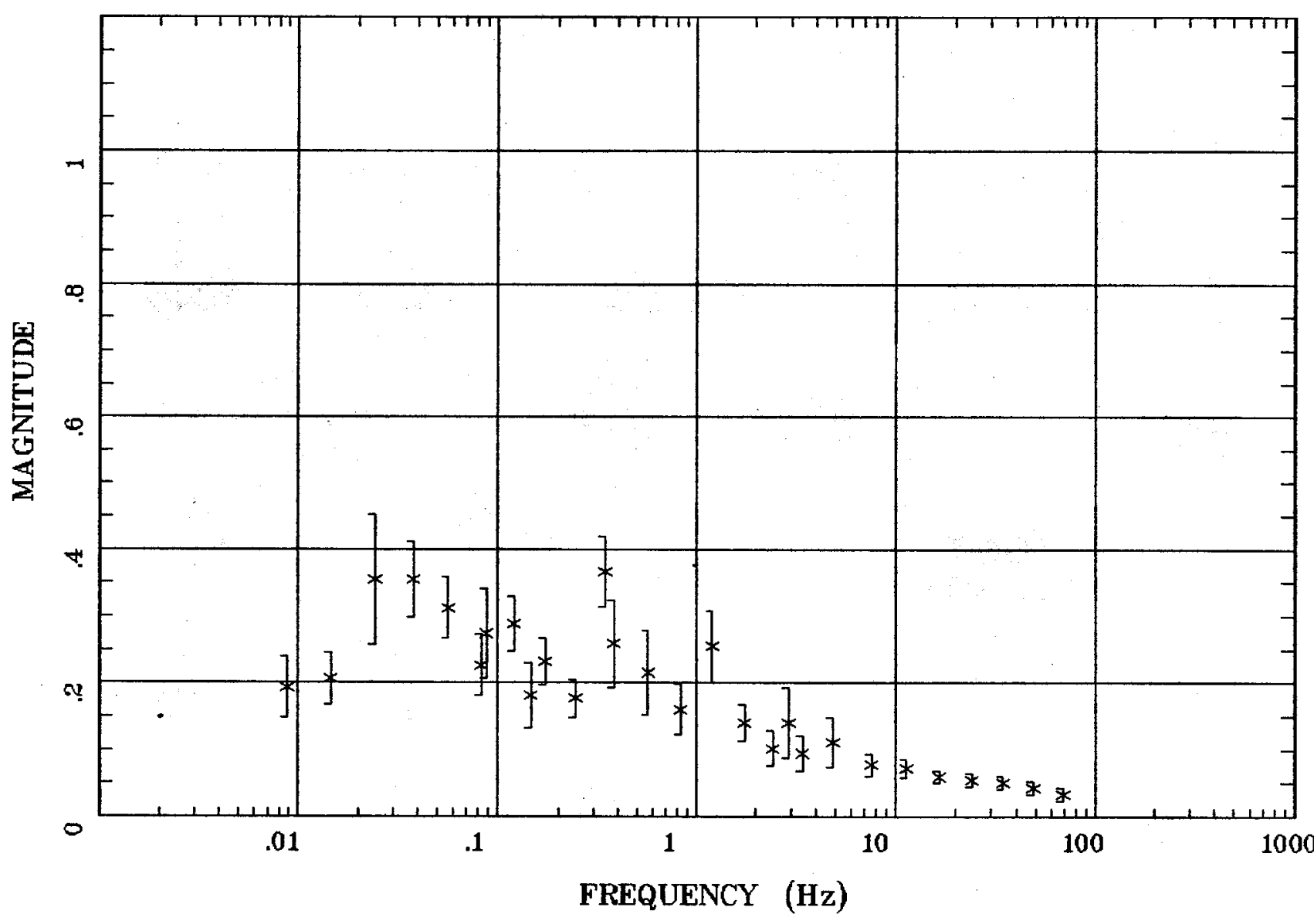

Client:

Remote: e-fld south $90 \mathrm{~m}$ Acquired: 10:1 Sep 05, 2003 Survey Co:USGS
Rotation:

Filename: ap03m.avg

Channels: Ch1 Ch2 Ch3 Ch4 Ch5 Ch6 Ch7 Plotted: 15:08 Sep 23, 2003

< EMI - ElectroMagnetic Instruments 
Wendover, NV 100kmap Station 3

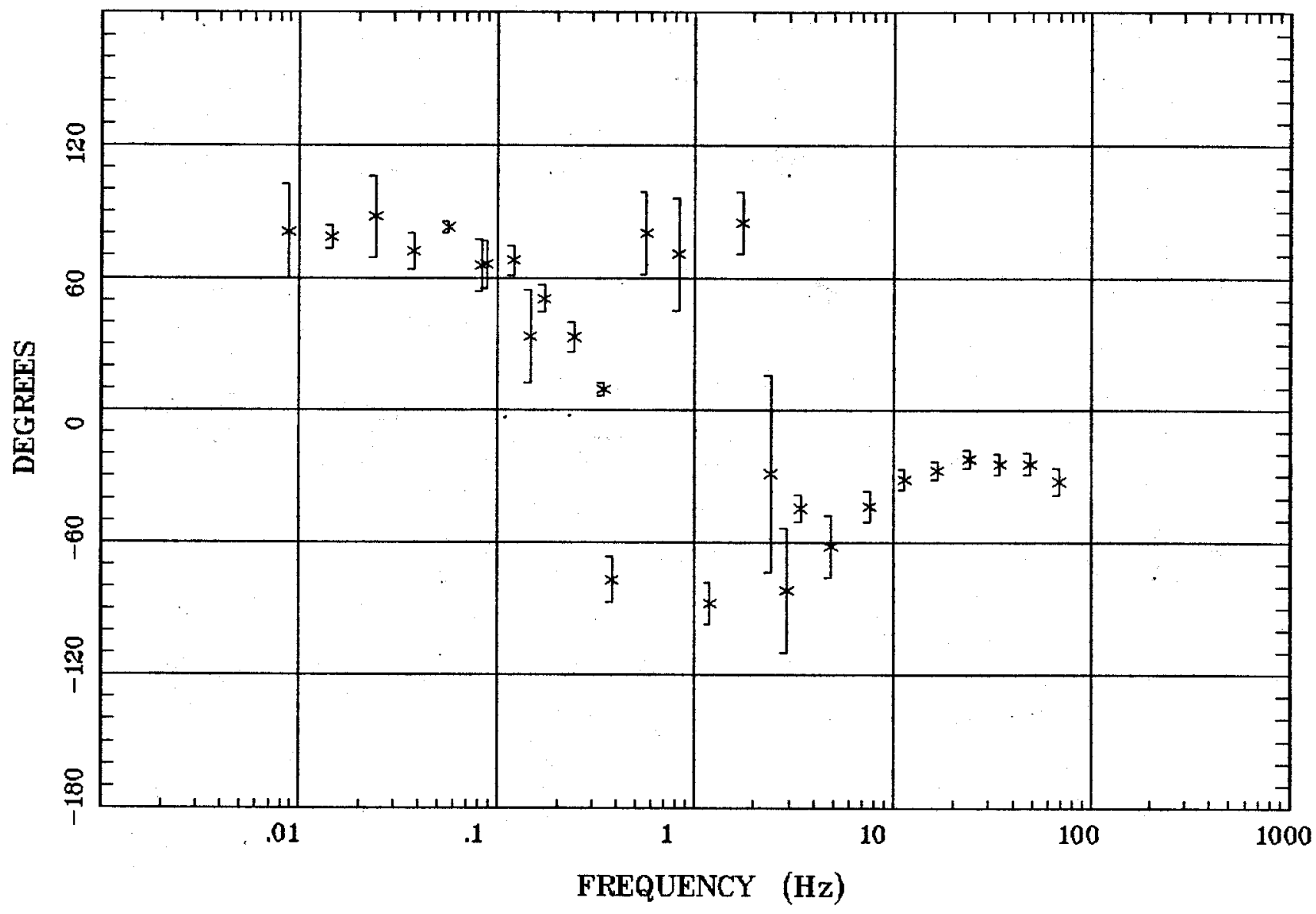

Client:

Remote: e-fld south $90 \mathrm{~m}$

Acquired: 10:1 Sep 05, 2003 Survey Co:USGS
Rotation:

Filename: ap03m.avg

Channels: Ch1 Ch2 Ch3 Ch4 Ch5 Ch6 Ch7 Plotted: 15:08 Sep 23, 2003

< EMI - ElectroMagnetic Instruments 
Station 3

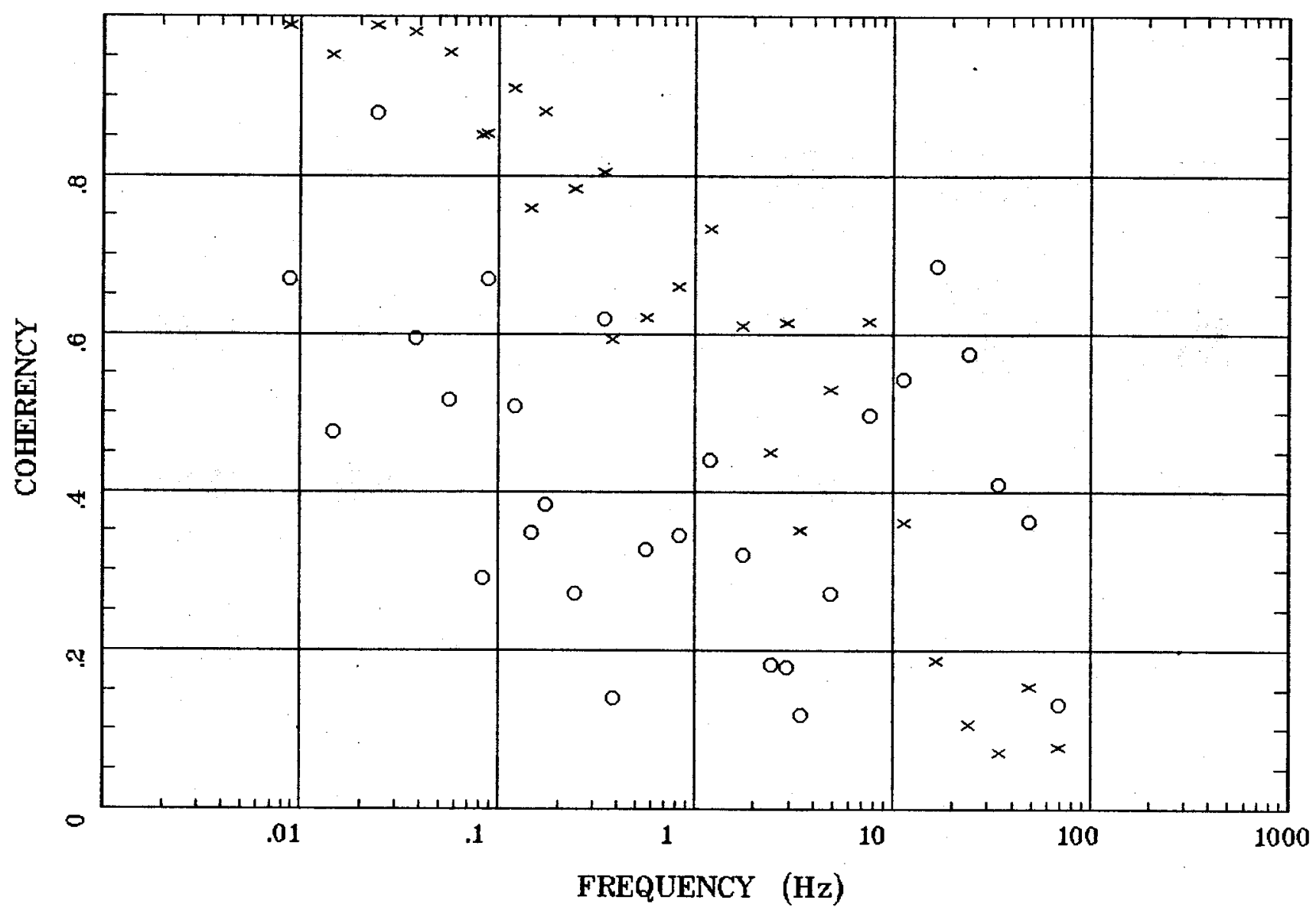

Client:

Remote: e-fld south $90 \mathrm{~m}$

Acquired: 10:1 Sep 05, 2003

Survey Co:USGS

Rotation:

Filename: ap03m.avg

Channels: Ch1 Ch2 Ch3 Ch4 Ch5 Ch6 Ch7

Plotted: 15:08 Sep 23, 2003

< EMI - ElectroMagnetic Instruments 


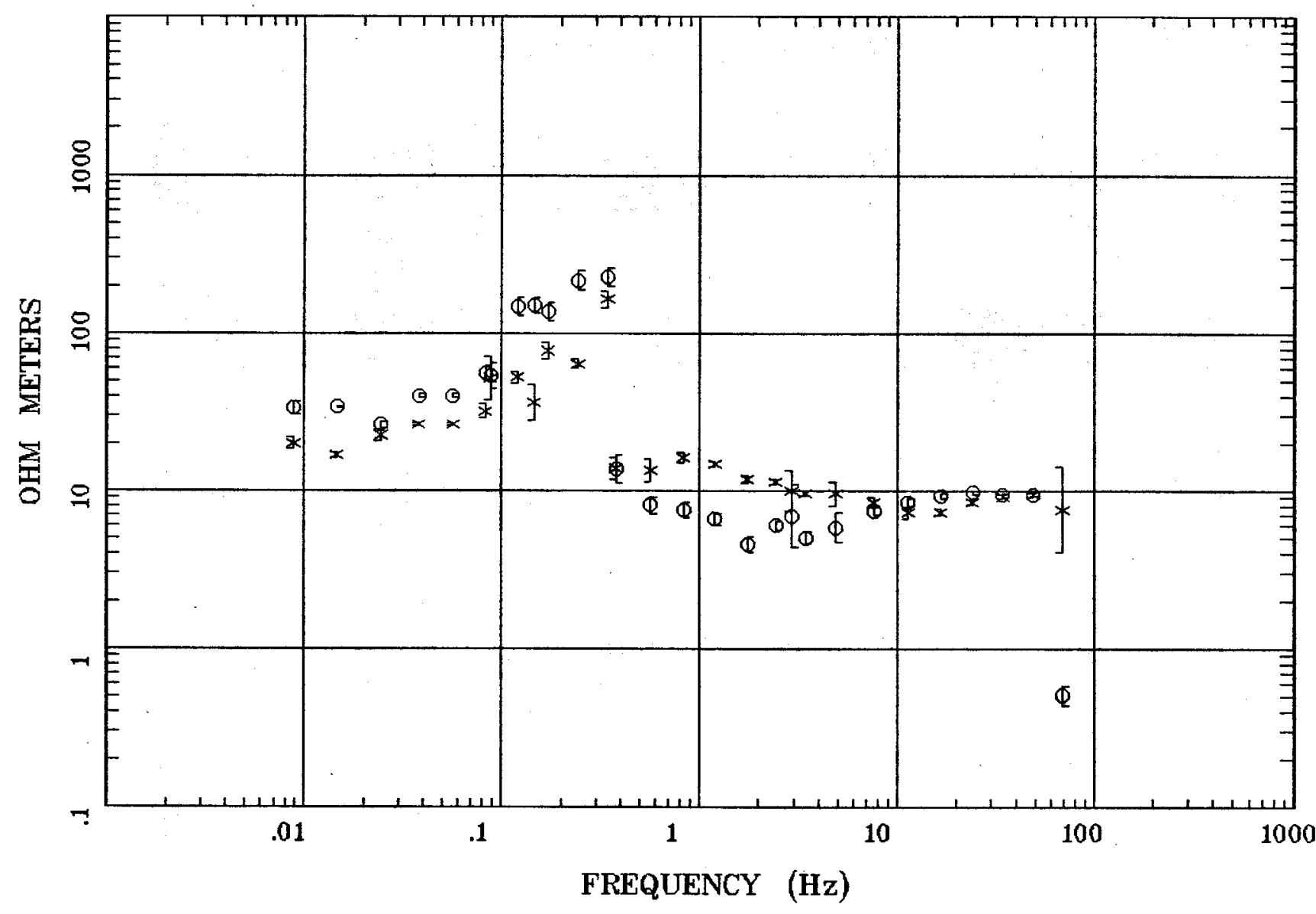

Client:

Remote: e-fld south $90 \mathrm{~m}$ Acquired: 14:0 Sep 05, 2003 Survey Co:USGS
Rotation:

Filename: ap04m.avg

Channels: Ch1 Ch2 Ch3 ch4 Ch5 Ch6 Ch7 Plotted: 08:28 Sep 24, 2003

< EMI - ElectroMagnetic Instruments > 
Wendover, NV 100kmap Station 4

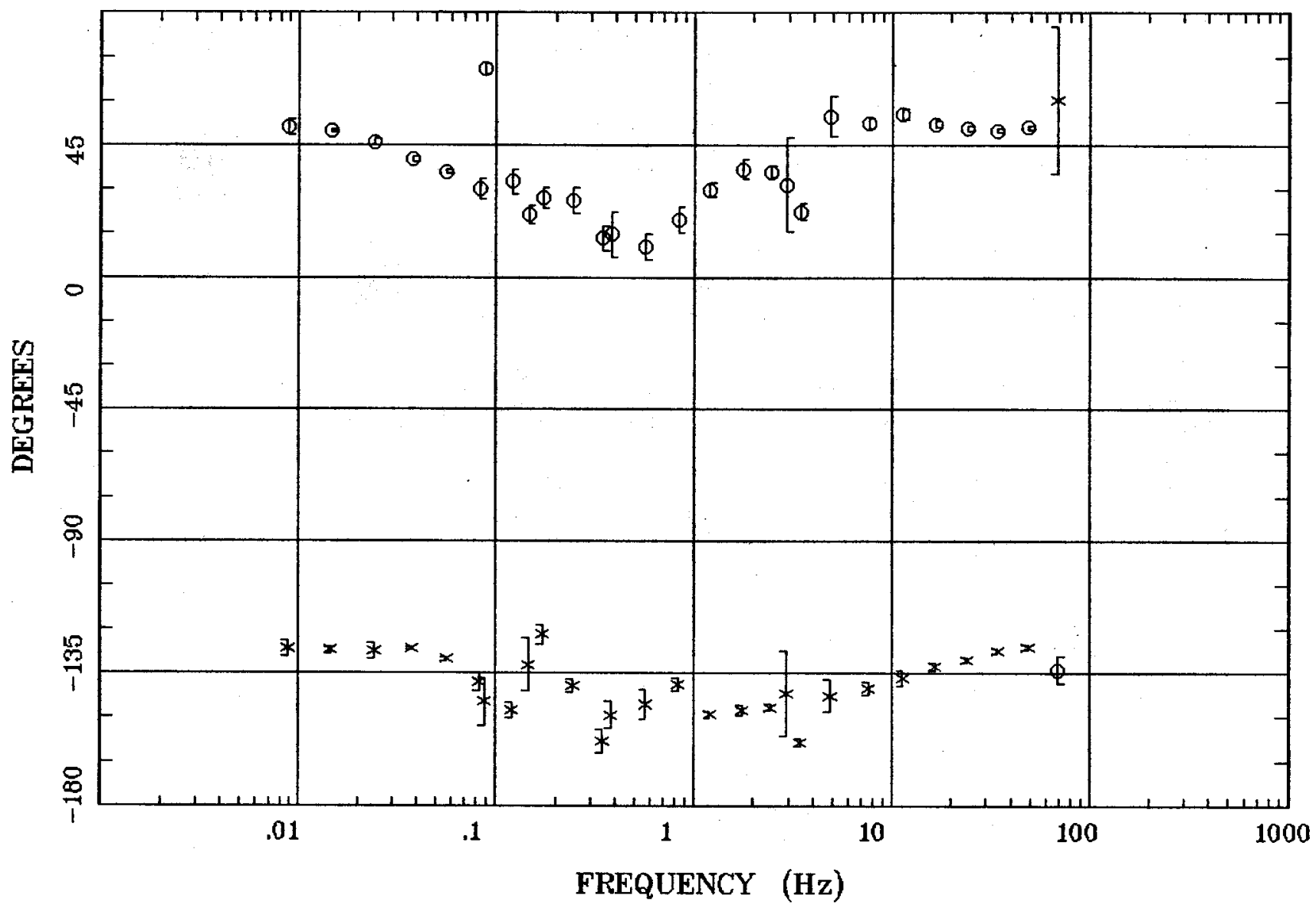

Client:

Remote: e-fld south $90 \mathrm{~m}$ Acquired: 14:0 Sep 05, 2003 Survey Co:USGS
Rotation:

Filename: ap04m.avg

Channels: Ch1 Ch2 Ch3 Ch4 Ch5 Ch6 Chr Plotted: 08:28 Sep 24, 2003

< EMI - ElectroMagnetic Instruments 


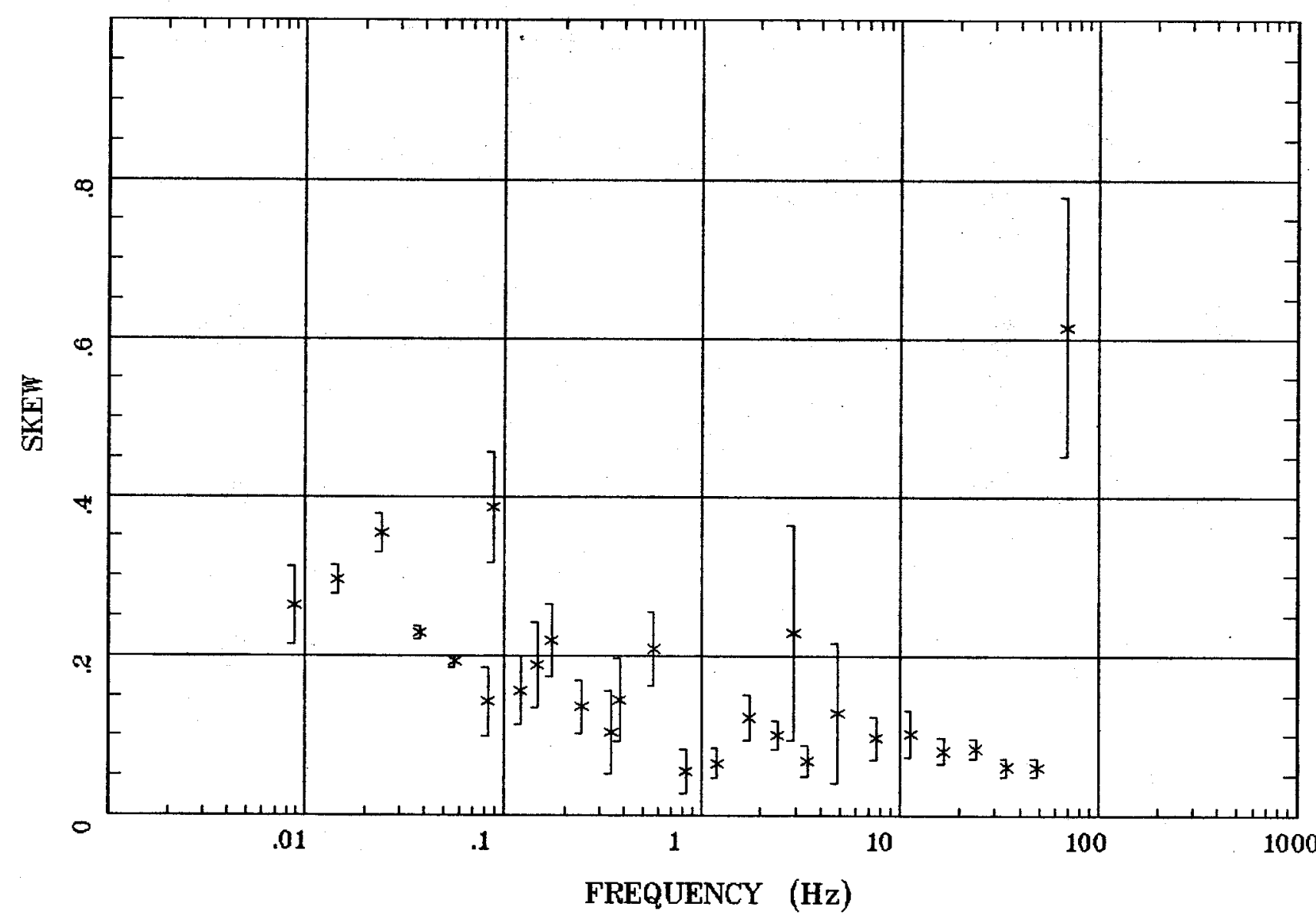

Client:

Remote: e-fld south $90 \mathrm{~m}$

Acquired: 14:0 Sep 05, 2003 Survey Co:USGS
Rotation:

Filename: ap04m.avg

Channels: Ch1 Ch2 Ch3 Ch4 Ch5 Ch6 Ch7 Plotted: 08:28 Sep 24, 2003

$<$ EMI - ElectroMagnetic Instruments 
E MULT Coh.

Wendover, NV 100kmap Station 4

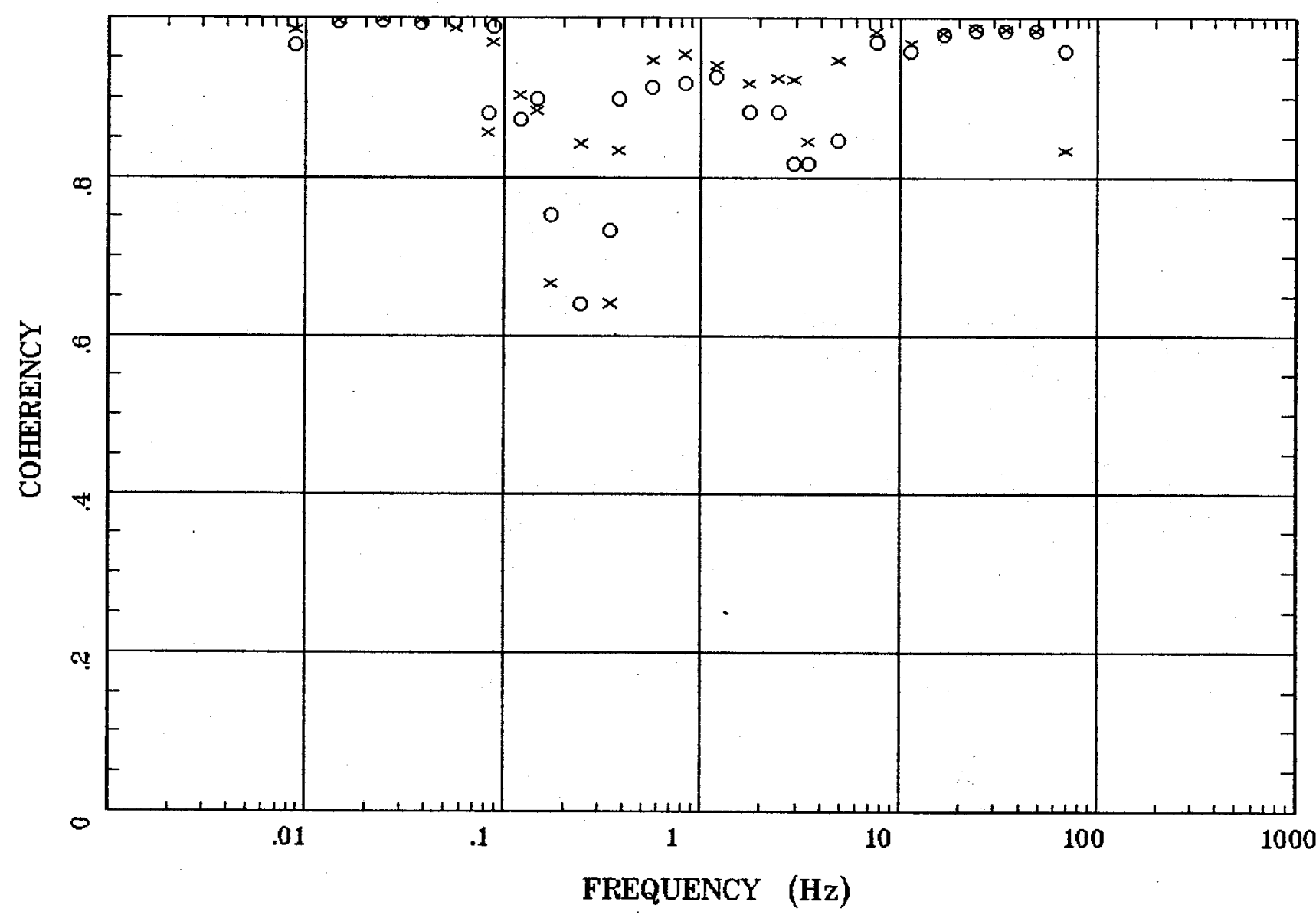

Client:

Remote: e-fld south $90 \mathrm{~m}$ Acquired: 14:0 Sep 05, 2003 Survey Co:USGS
Rotation:

Filename: ap04m.avg

Channels: Ch1 Ch2 Ch3 Ch4 Ch5 Ch6 Ch7 Plotted: 08:28 Sep 24, 2003

$<$ EMI - ElectroMagnetic Instruments > 


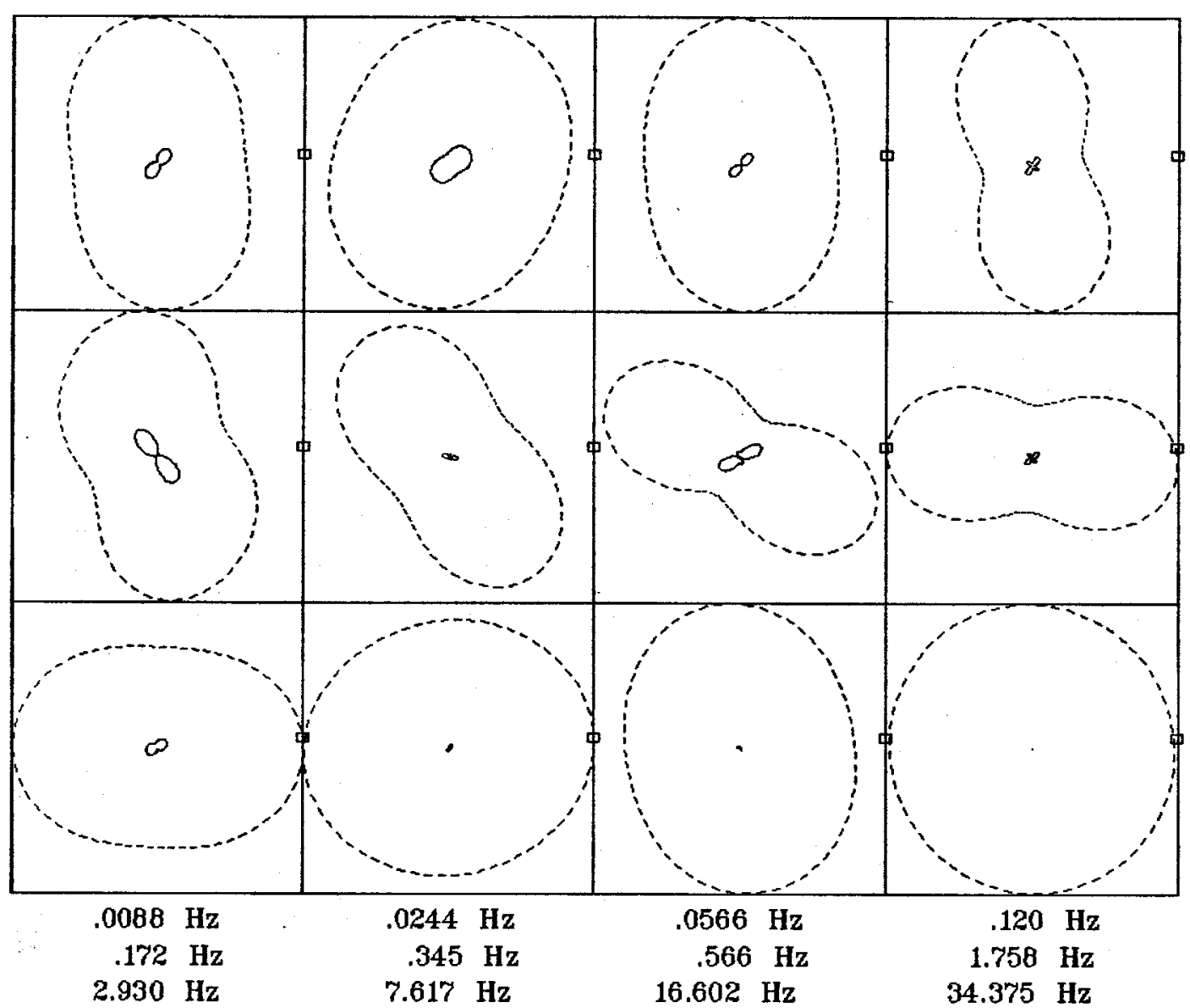

Client:

Remote: e-fld south $90 \mathrm{~m}$ Acquired: 14:0 Sep 05, 2003 Survey Co:USGS
Rotation:

Filename: ap04m.avg

Channels: Ch1 Ch2 Ch3 Ch4 Ch5 Ch6 Ch7 Plotted: 08:28 Sep 24, 2003

< EMI - ElectroMagnetic Instruments > 


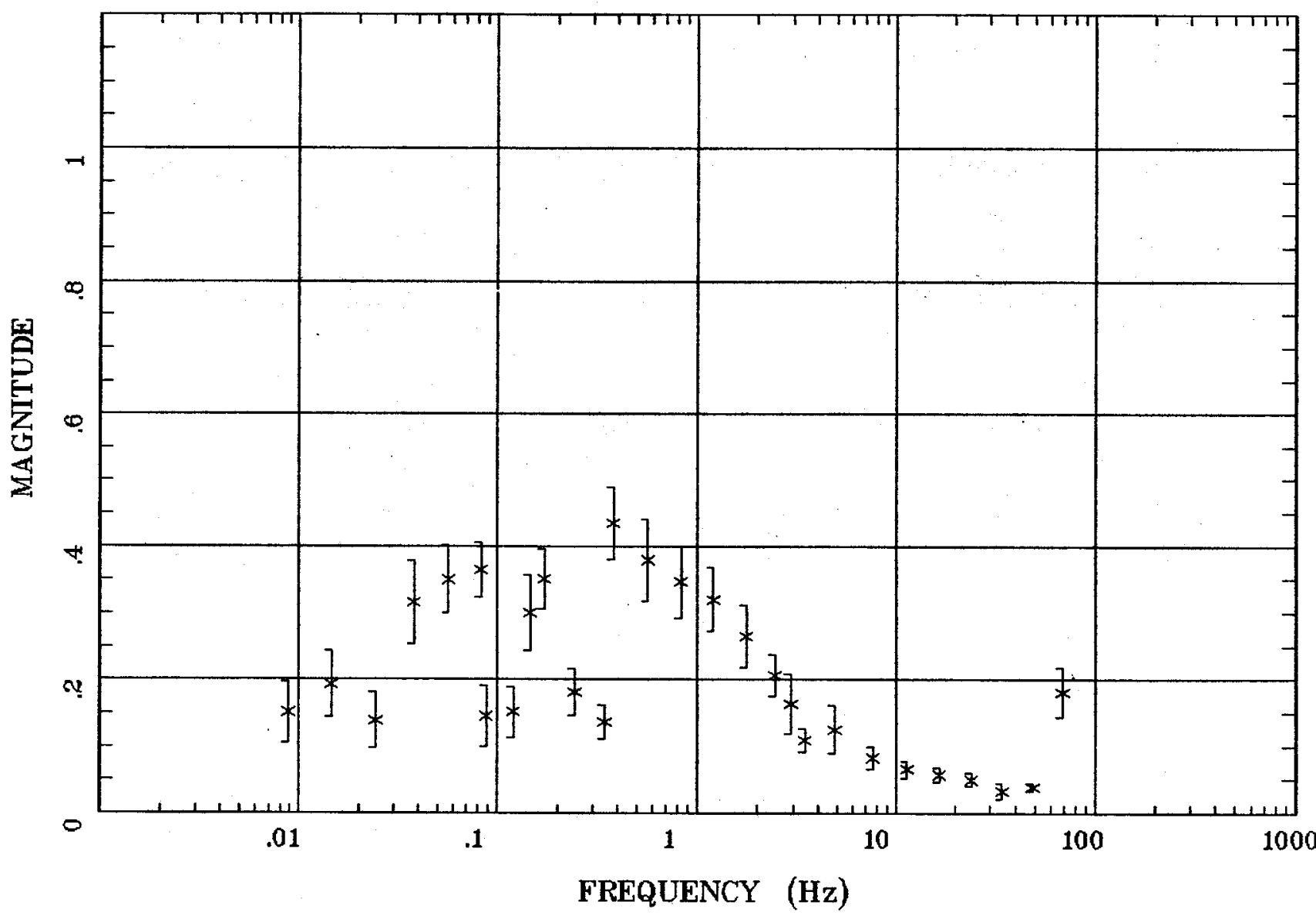

Client:

Remote: e-fld south $90 \mathrm{~m}$ Acquired: 14:0 Sep 05, 2003 Survey Co:USGS
Rotation:

Filename: ap04m.avg

Channels: Ch1 Ch2 Ch3 Ch4 Ch5 Ch6 Ch7 Plotted: 08:28 Sep 24, 2003

< EMI - ElectroMagnetic Instruments 


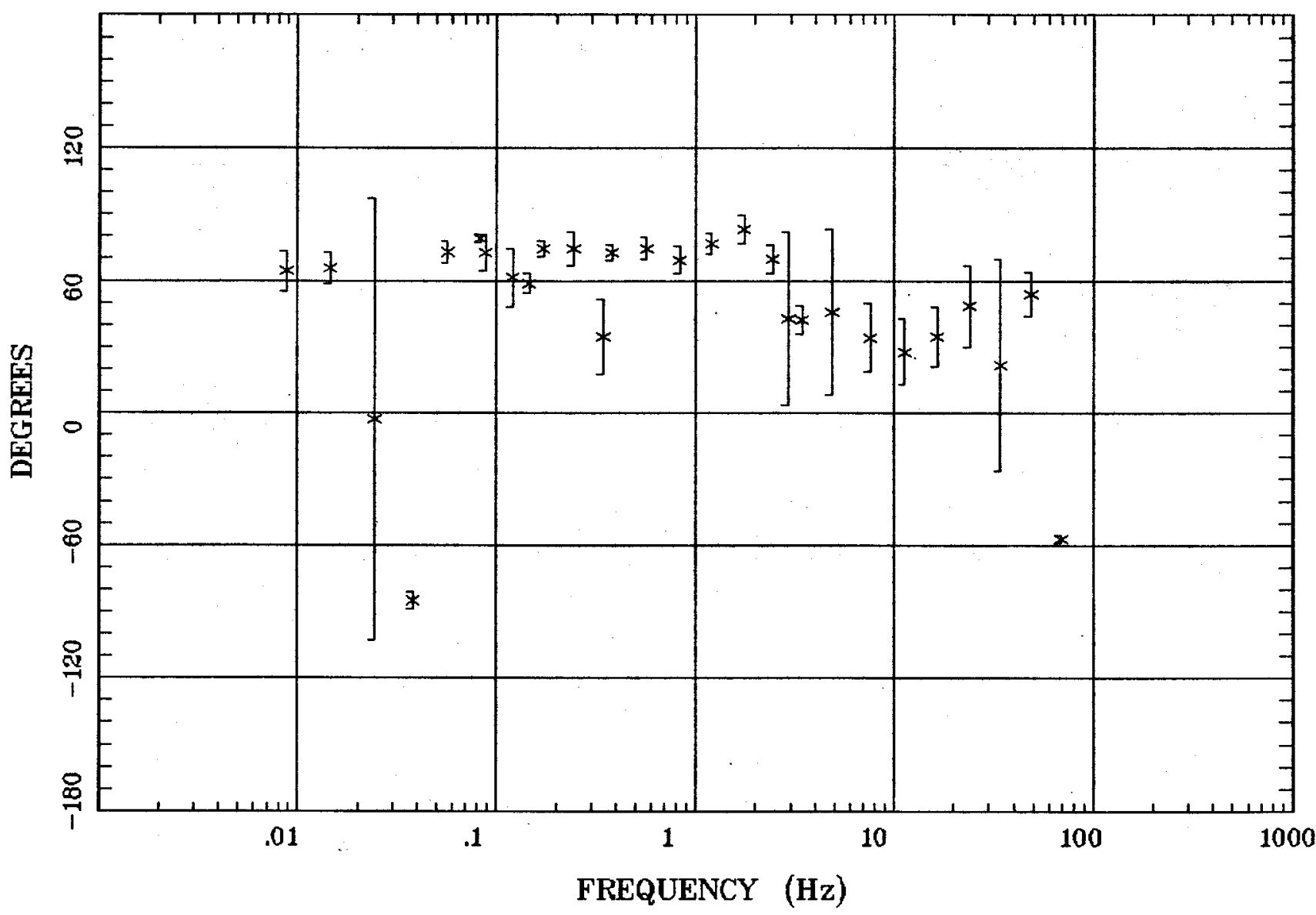

Client:

Remote: e-fld south $90 \mathrm{~m}$ Acquired: 14:0 Sep 05, 2003 Survey Co:USGS
Rotation:

Filename: ap04m.avg

Channels: Ch1 Ch2 Ch3 Ch4 ch5 Ch6 Ch7 Plotted: 08:28 Sep 24, 2003

< EMI - ElectroMagnetic Instruments 


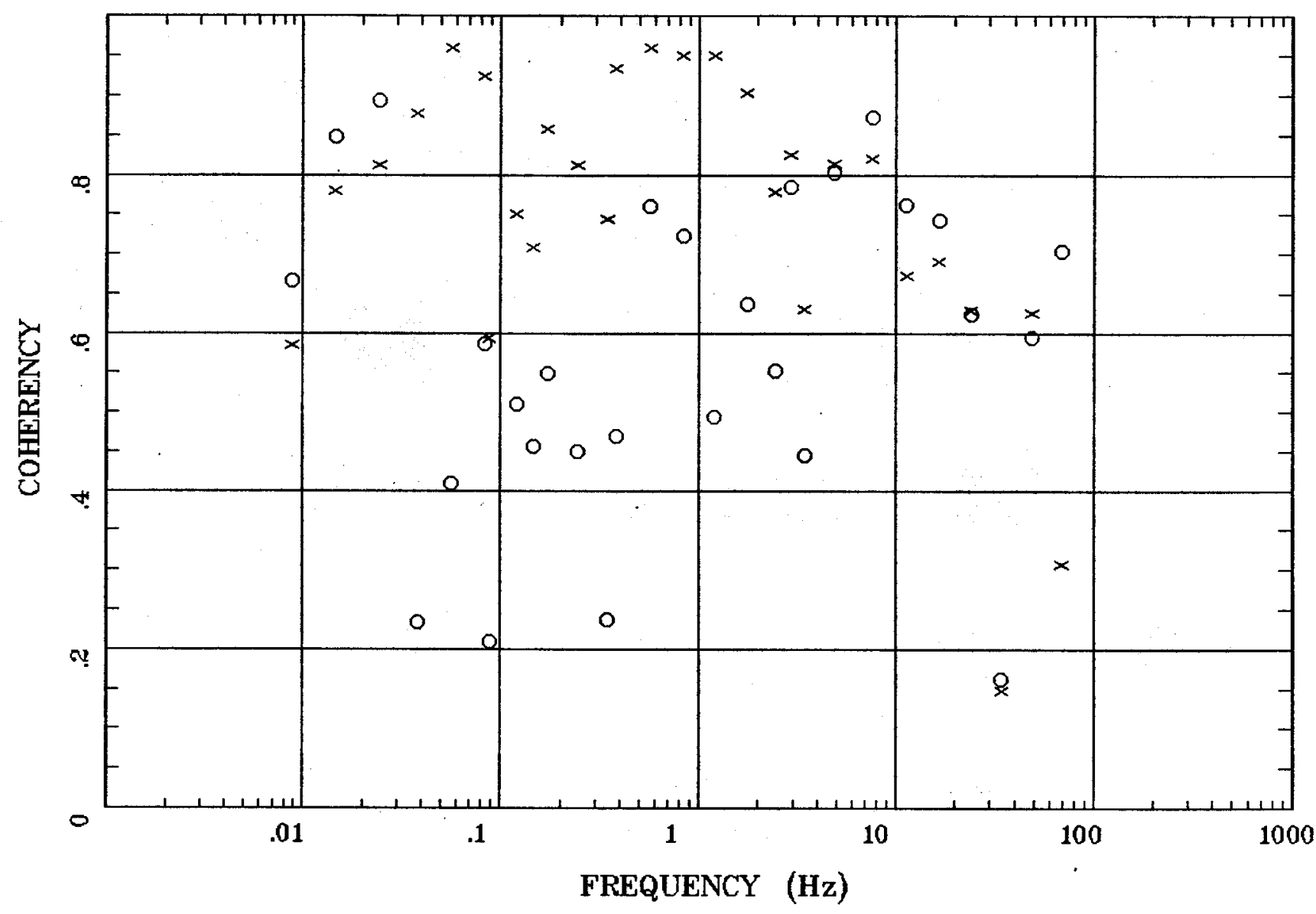

Client:

Remote: e-fld south $90 \mathrm{~m}$ Acquired: 14:0 Sep 05, 2003 Survey Co:USGS
Rotation:

Filename: ap04m.avg

Channels: Ch1 Ch2 Ch3 Ch4 Ch5 Ch6 Ch7 Plotted: 08:28 Sep 24, 2003

$<$ EMI - ElectroMagnetic Instruments > 


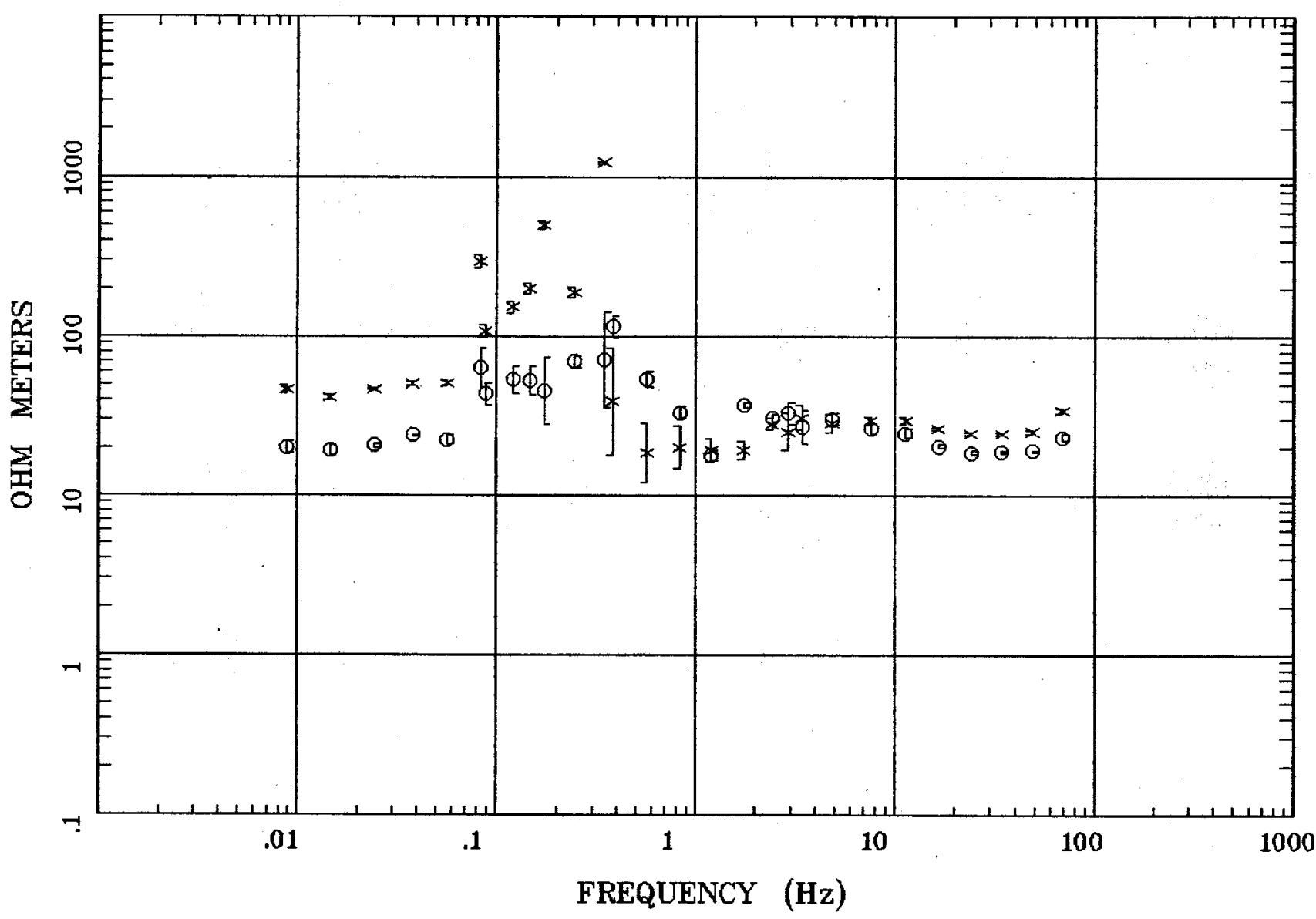

Client:

Remote: e-fld south $90 \mathrm{~m}$ Acquired: 09:5 Sep 06, 2003 Survey Co:USGS
Rotation:

Filename: ap05m.avg

Channels: Ch1 Ch2 Ch3 Ch4 Ch5 Ch6 Ch7 Plotted: 08:29 Sep 24, 2003

< EMI - ElectroMagnetic Instruments 
Wendover, NV 100kmap Station 5

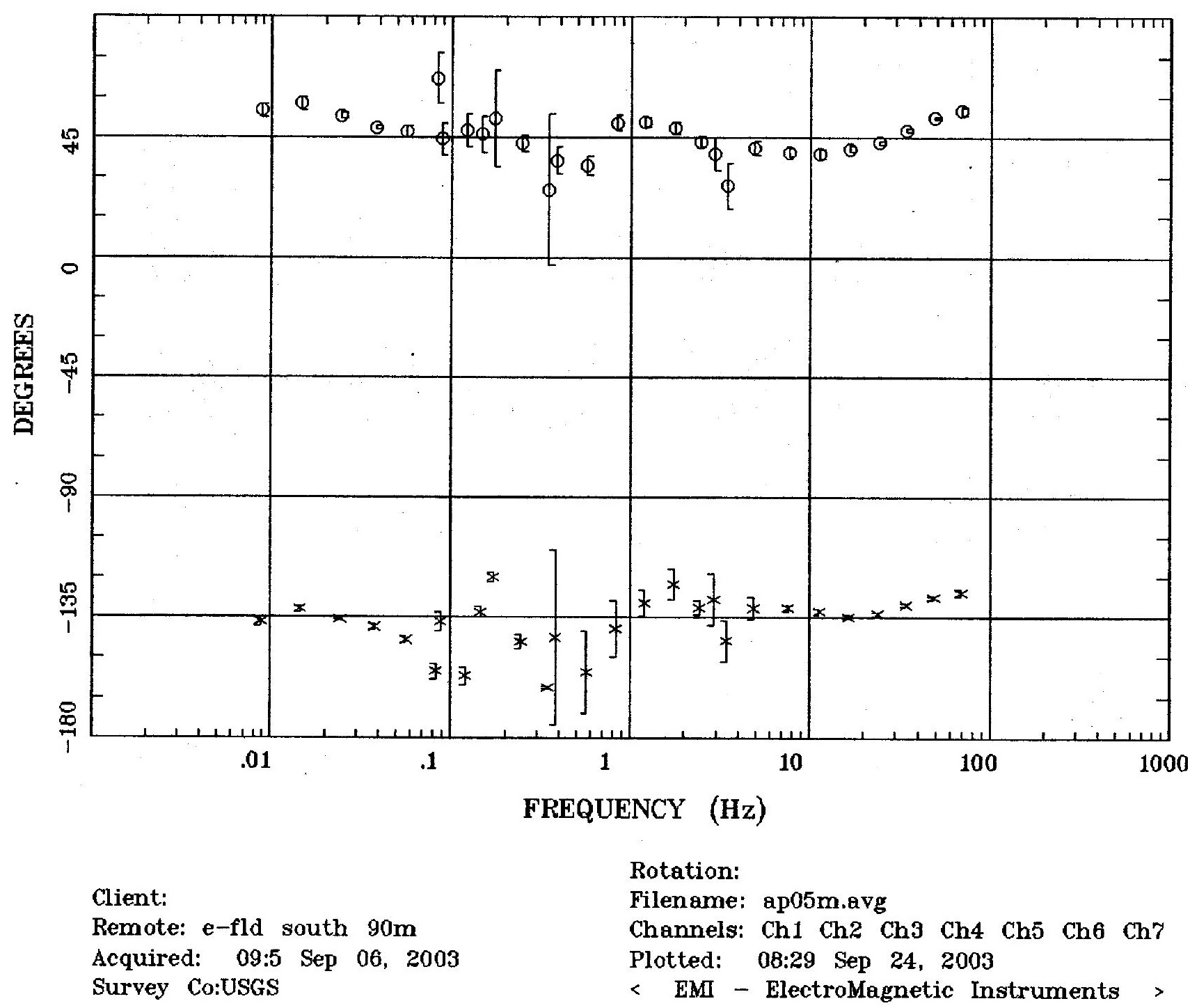


Wendover, NV 100kmap Station 5

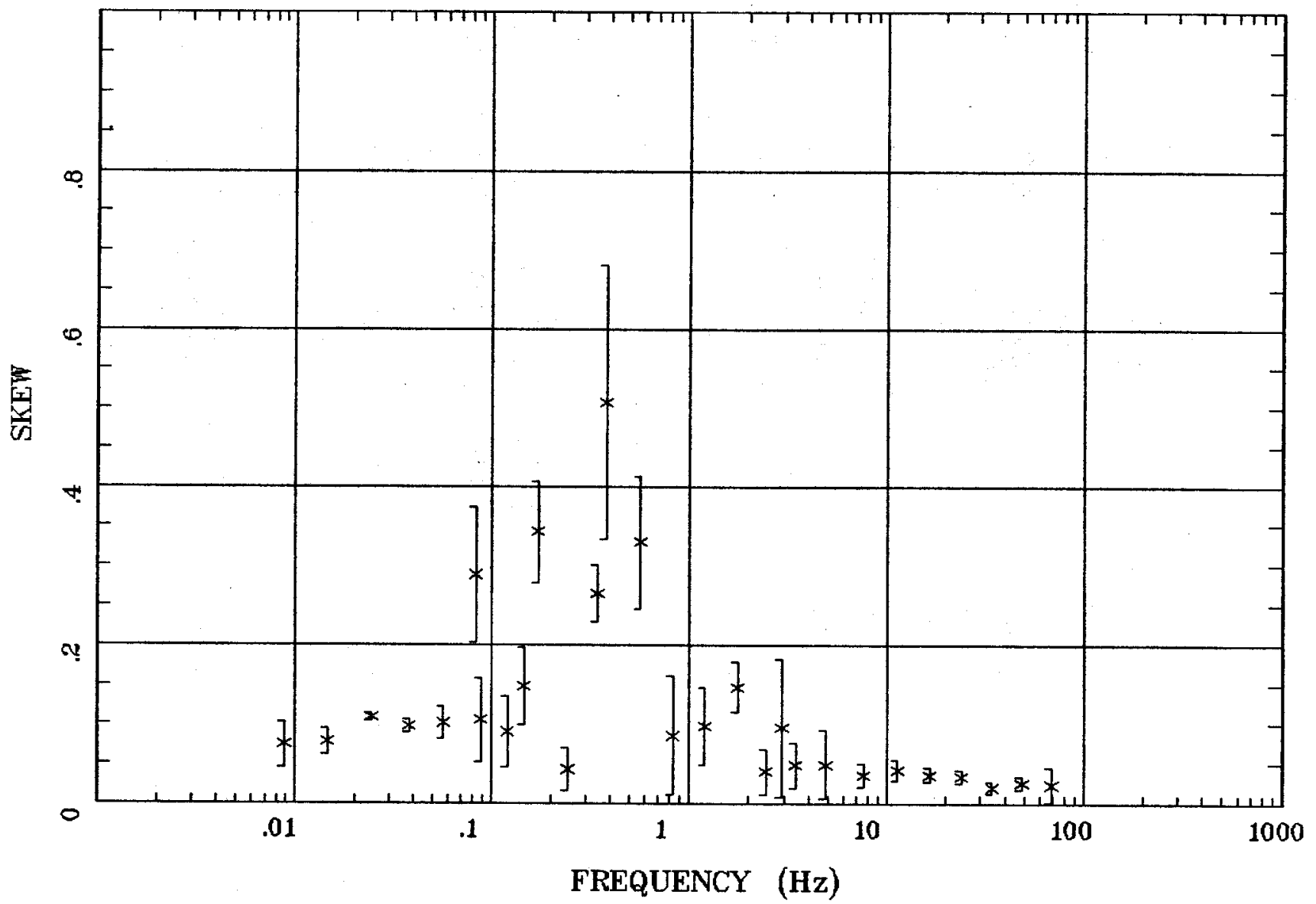

Client:

Rotation:

Remote: e-fld south $90 \mathrm{~m}$

Acquired: 09:5 Sep 06, 2003

Filename: ap05m.avg

Channels: Ch1 Ch2 Ch3 Ch4 Ch5 Ch6 Ch7

Survey Co:USGS

Plotted: 08:29 Sep 24, 2003

$<$ EMI - ElectroMagnetic Instruments 


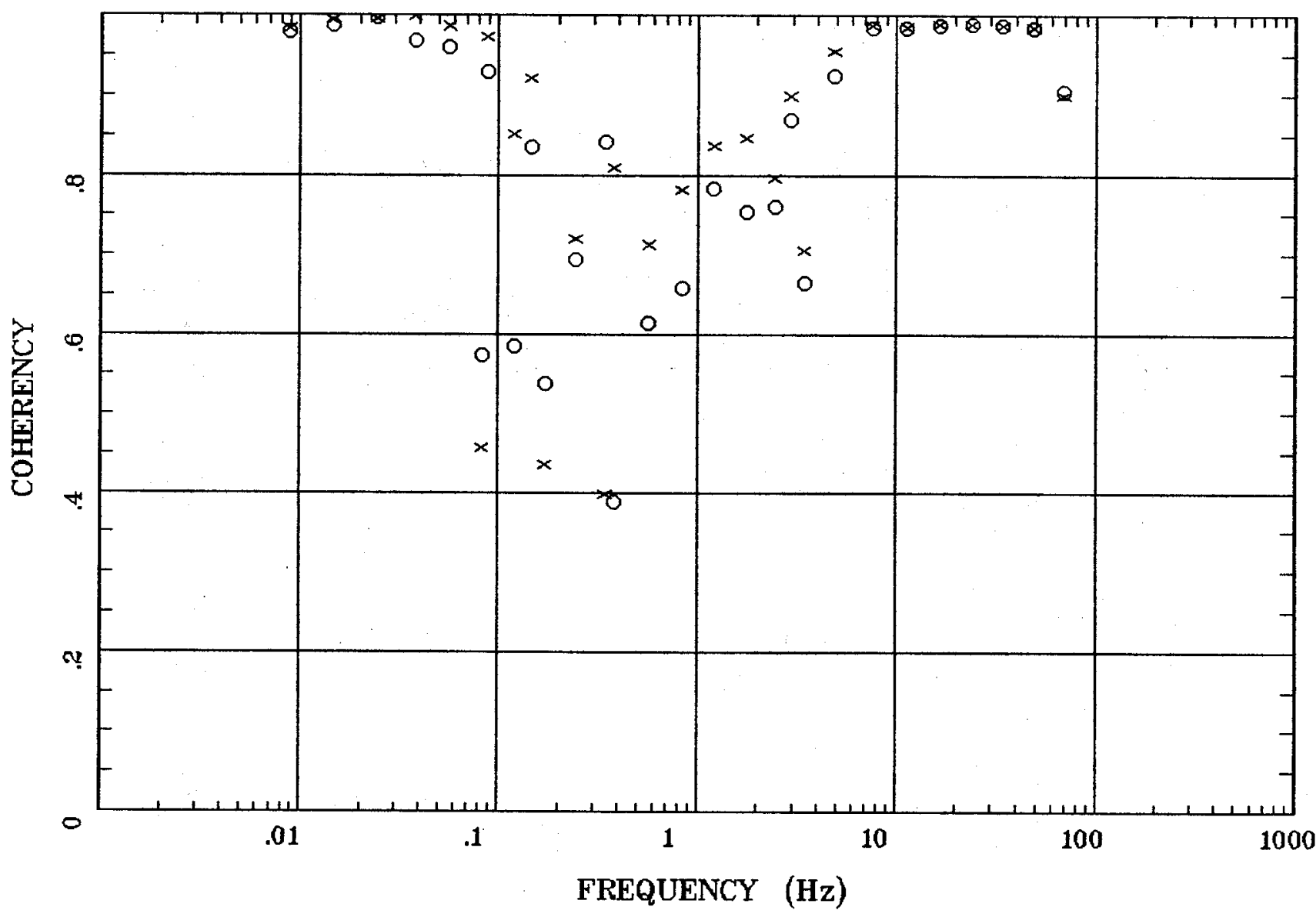

Client:

Remote: e-fld south $90 \mathrm{~m}$ Acquired: 09:5 Sep 06, 2003 Survey Co:USGS
Rotation:

Filename: ap05m.avg Channels: Ch1 Ch2 Ch3 Ch4 Ch5 Ch6 Ch7 Plotted: 08:29 Sep 24, 2003

$<$ EMI - ElectroMagnetic Instruments 


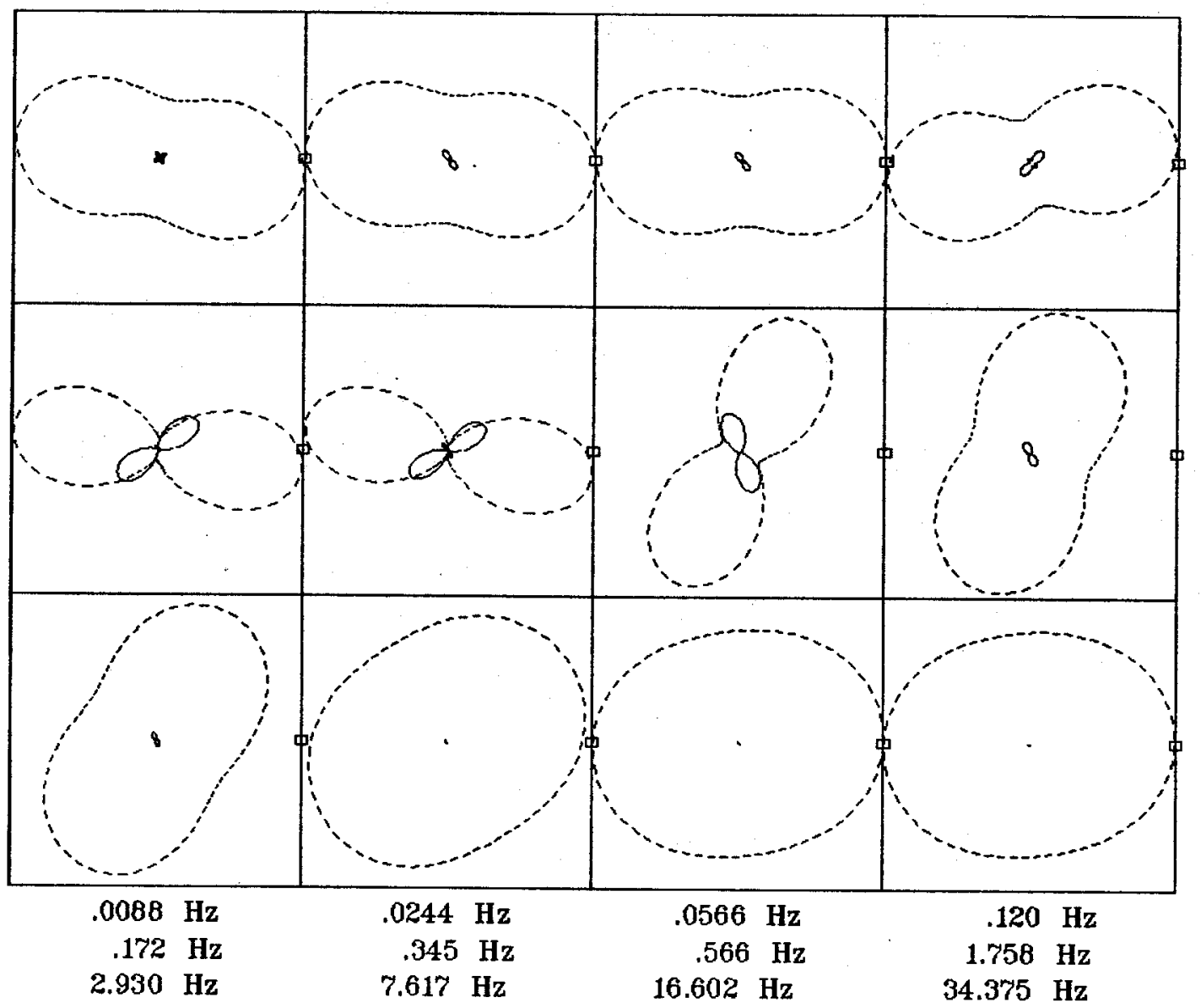

Client:

Remote: e-fld south $90 \mathrm{~m}$ Acquired: 09:5 Sep 06, 2003 Survey Co:USGS

\section{Rotation:}

Filename: ap05m.avg

Channels: Ch1 Ch2 Ch3 Ch4 Ch5 Ch6 Ch7 Platted: 08:29 Sep 24, 2003

$\langle$ EMI - ElectroMagnetic Instruments > 


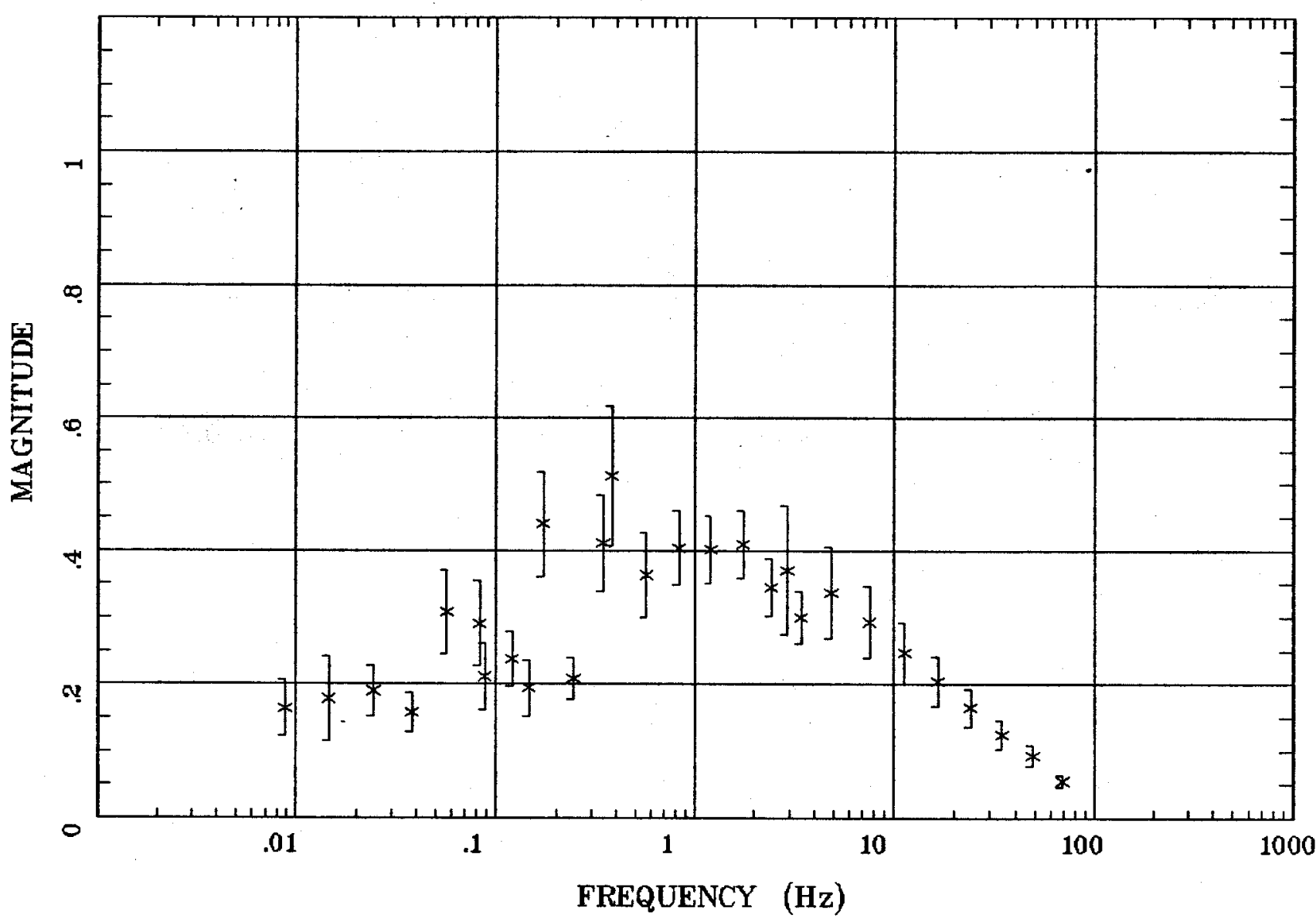

Client:

Remote: e-fld south $90 \mathrm{~m}$ Acquired: 09:5 Sep 06, 2003 Survey Co:USGS

\section{Rotation:}

Filename: ap05m.avg

Channels: Ch1 Ch2 Ch3 Ch4 Ch5 Ch6 Ch7 Plotted: 08:29 Sep 24, 2003

< EMI - ElectroMagnetic Instruments > 


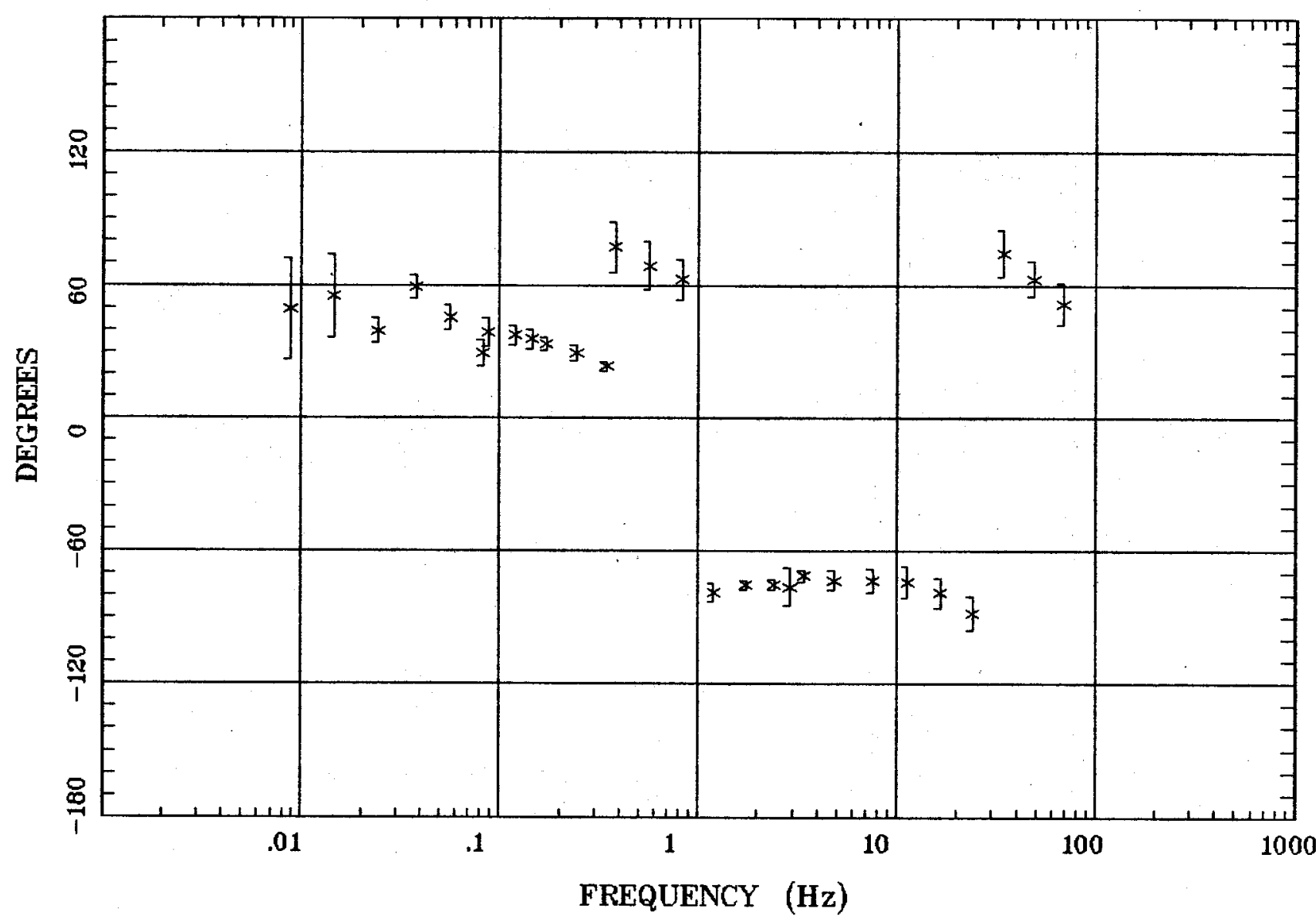

Client:

Remote: e-fld south $90 \mathrm{~m}$ Acquired: 09:5 Sep 06, 2003 Survey Co:USGS
Rotation:

Filename: ap05m.avg

Channels: Ch1 Ch2 Ch3 Ch4 Ch5 Ch6 Chr Plotted: 08:29 Sep 24, 2003

< EMI - ElectroMagnetic Instruments 
HzHx.x Coh HzHy.o

Wendover, NV 100kmap Station 5

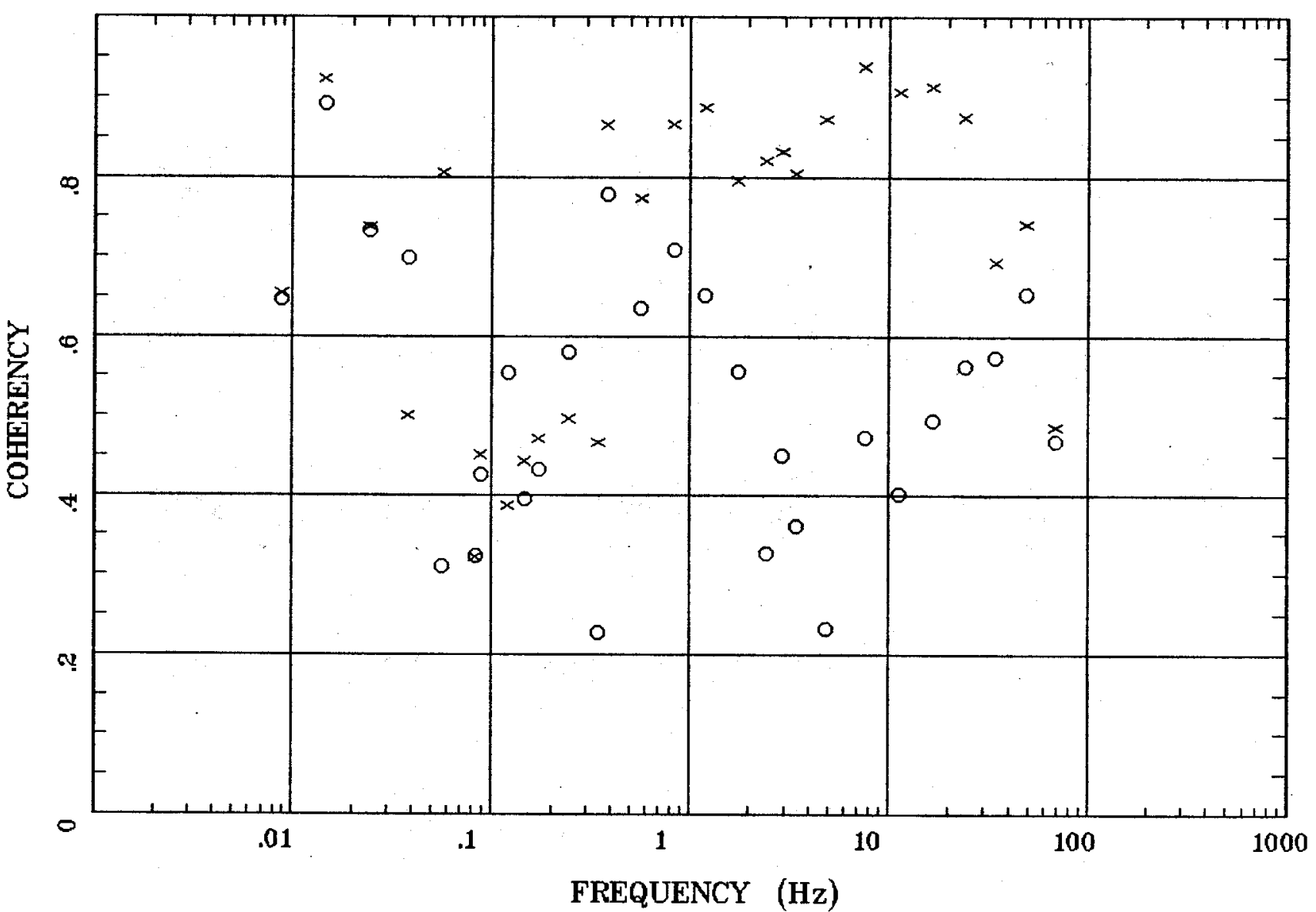

Client:

Rotation:

Remote: e-fld south $90 \mathrm{~m}$

Acquired: 09:5 Sep 06, 2003

Filename: ap05m.avg

Survey Co:USGS

Channels: Ch1 Ch2 Ch3 Ch4 Ch5 Ch6 Ch7

Plotted: 08:29 Sep 24, 2003

< EMI - ElectroMagnetic Instruments > 


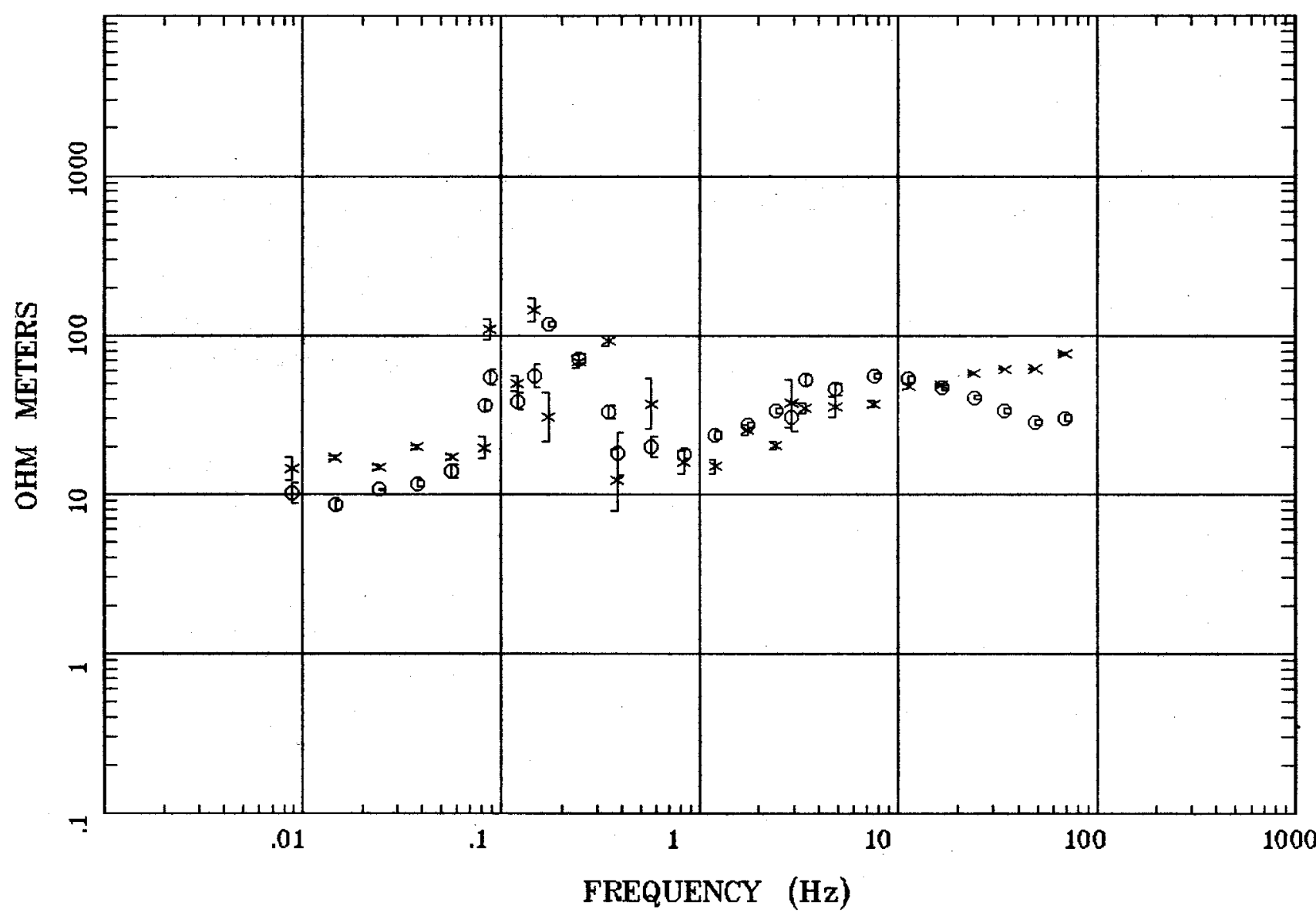

Client:

Remote: e-fld north $90 \mathrm{~m}$ Acquired: $13: 4$ Sep 06, 2003 Survey Co:USGS
Rotation:

Filename: ap06m.avg

Channels: Ch1 Ch2 Ch3 ch4 ch5 Ch6 Chr Plotted: $08: 30$ Sep 24, 2003

$<$ EMI - ElectroMagnetic Instruments 


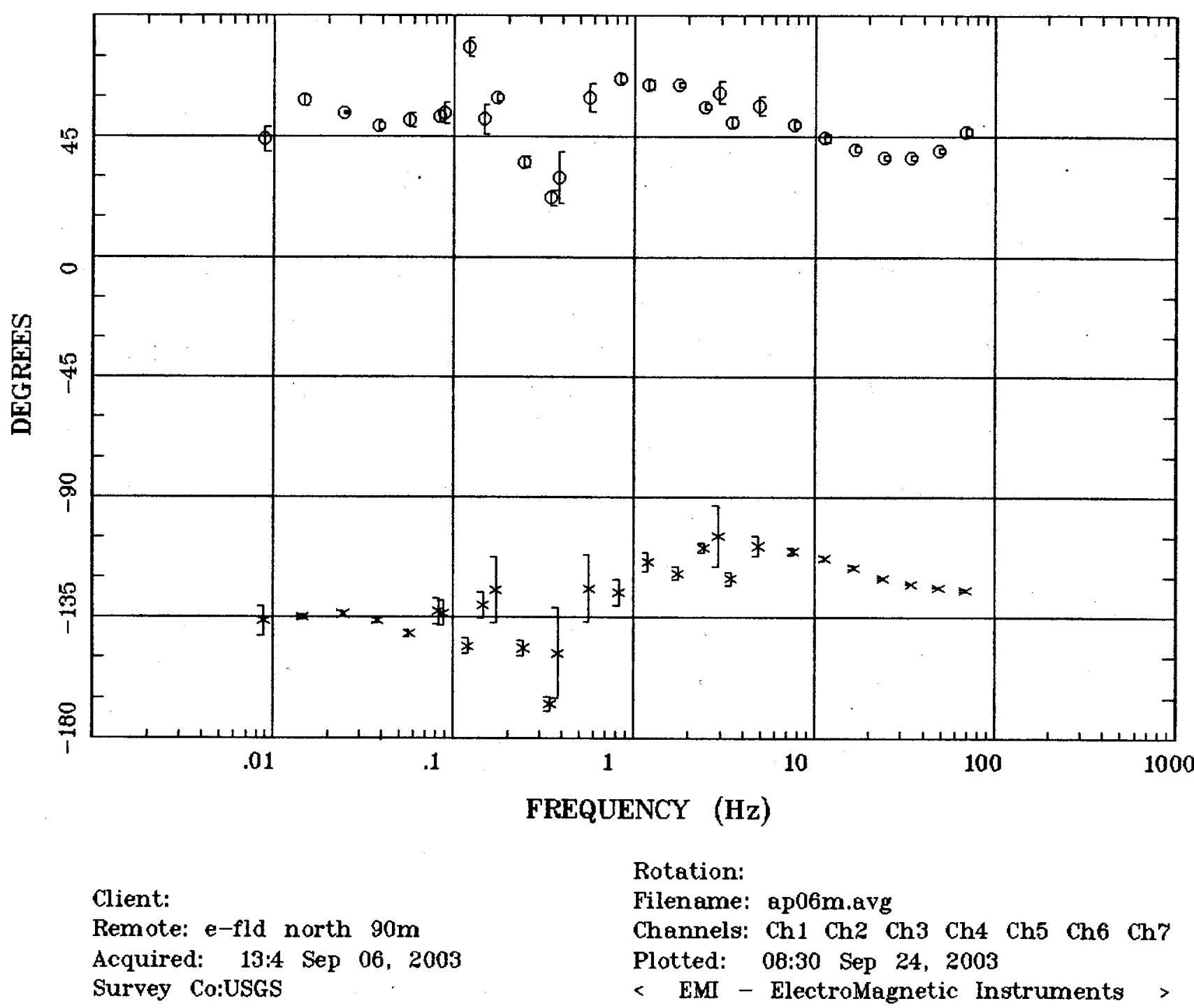




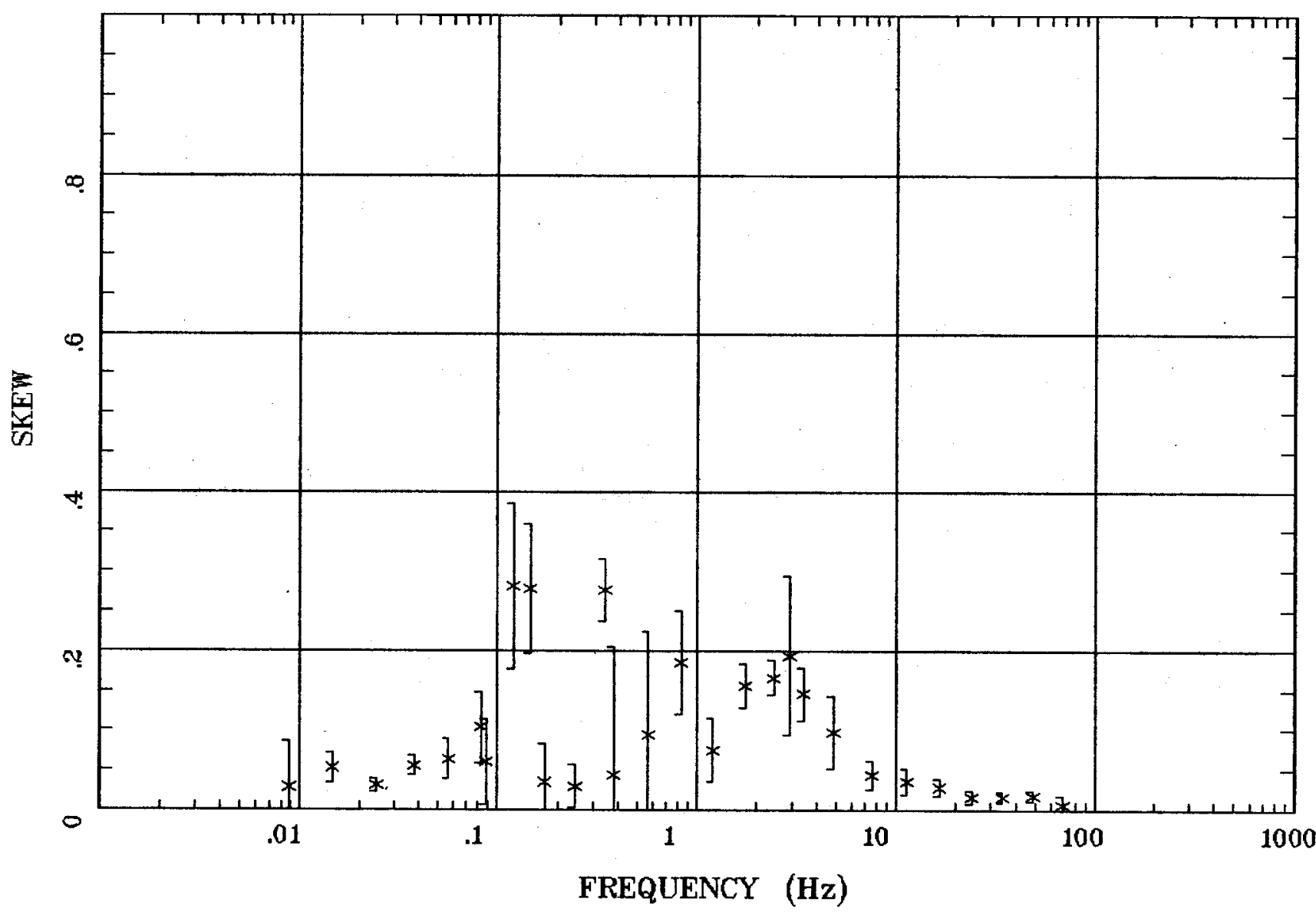

Client:

Remote: e-fld north $90 \mathrm{~m}$ Acquired: 13:4 Sep 06, 2003 Survey Co:USGS
Rotation:

Filename: ap06m.avg

Channels: Ch1 Ch2 Ch3 Ch4 Ch5 Ch6 Ch7

Plotted: 08:30 Sep 24, 2003

$<$ EMI - ElectroMagnetic Instruments 


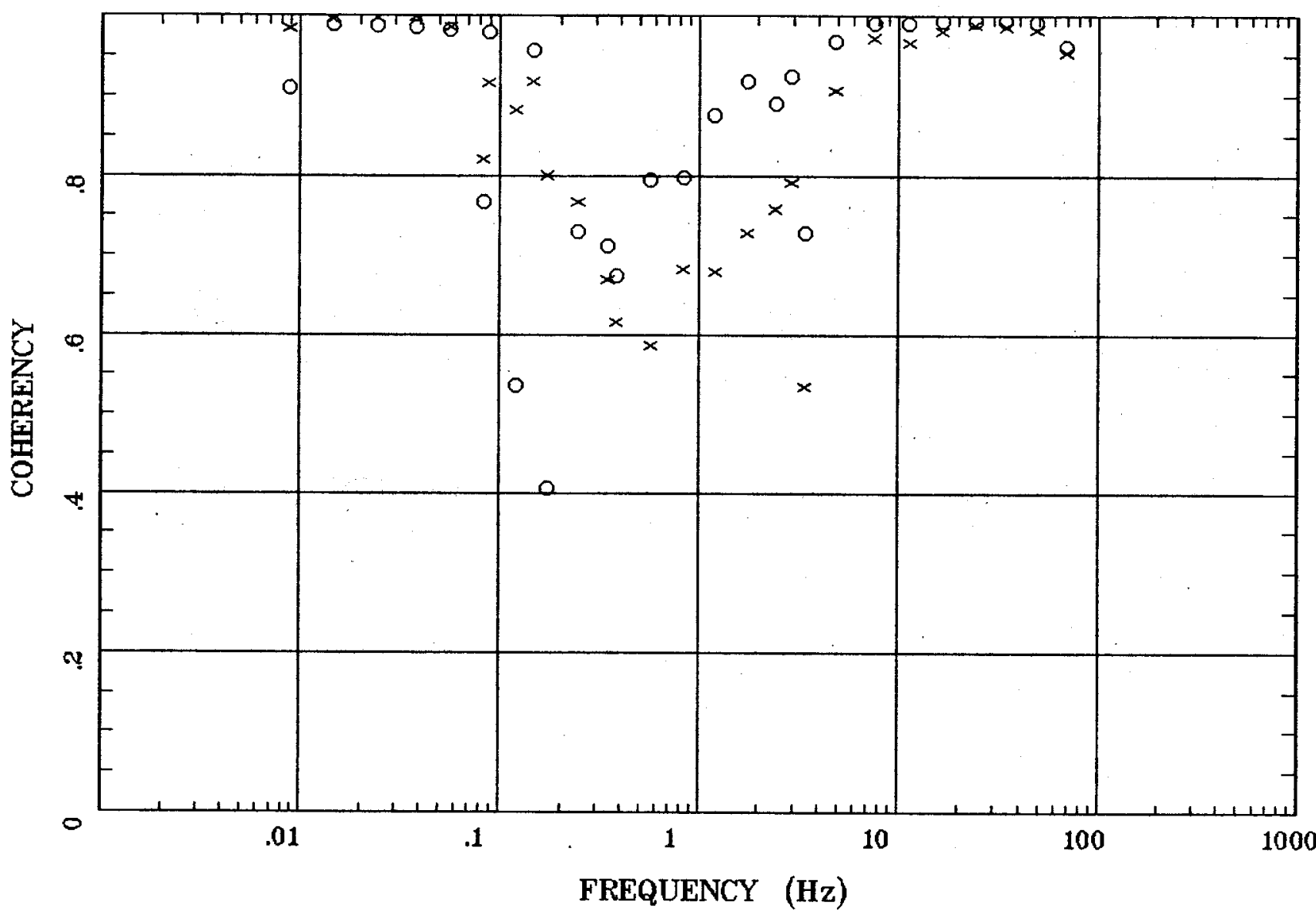

Client:

Remote: e-fld north $90 \mathrm{~m}$ Acquired: 13:4 Sep 06, 2003 Survey Co:USGS
Rotation:

Filename: ap06m.avg

Channels: Ch1 Ch2 Ch3 Ch4 Ch5 Ch6 Ch7 Plotted: 08:30 Sep 24, 2003

< EMI - ElectroMagnetic Instruments 


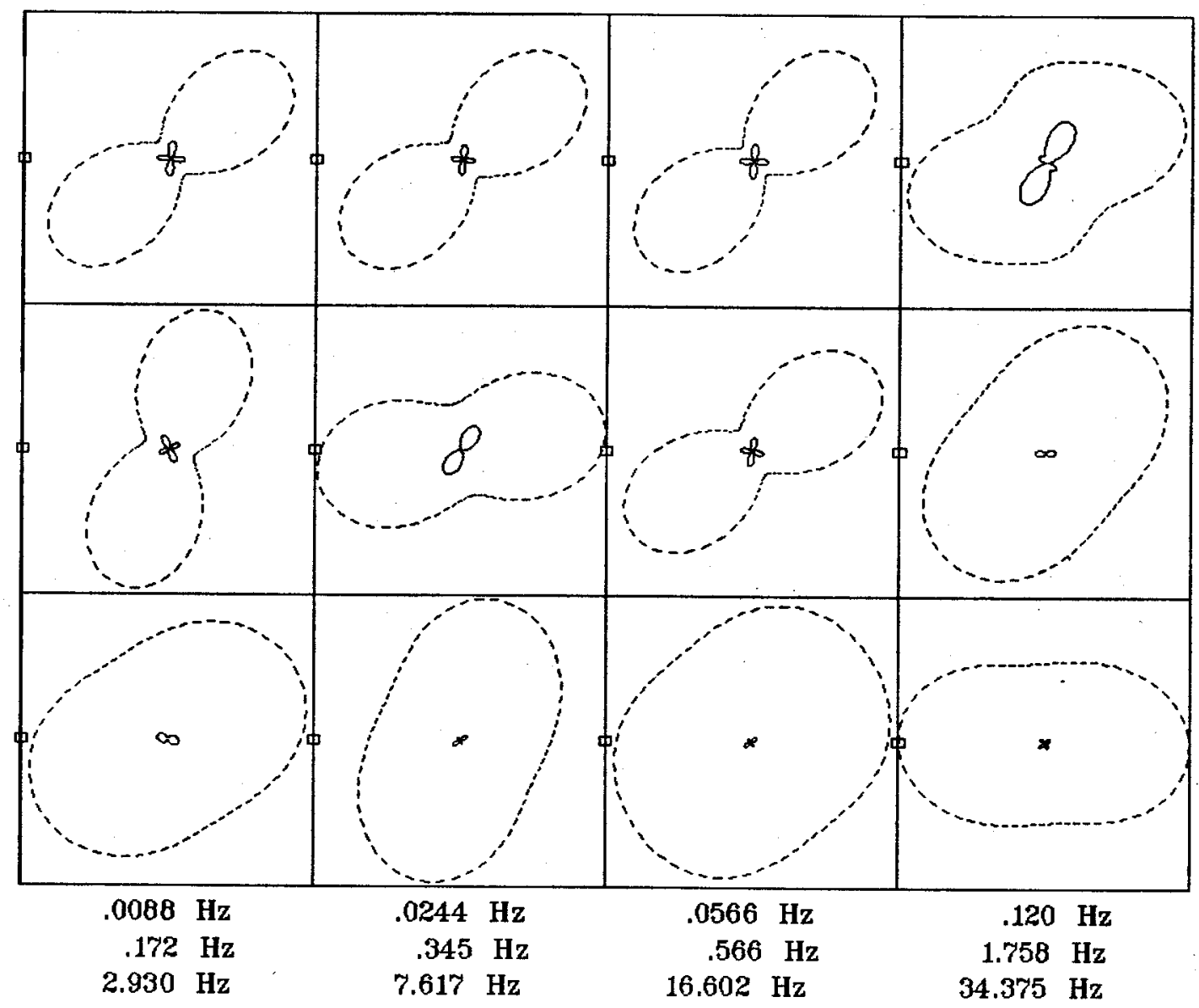

Client:

Remote: e-fld north $90 \mathrm{~m}$ Acquired: 13:4 Sep 06, 2003 Survey Co:USGS
Rotation:

Filename: ap06m.arg

Channels: Ch1 Ch2 Ch3 Ch4 Ch5 Ch6 Ch7 Plotted: 08:30 Sep 24, 2003

$<$ EMI - ElectroMagnetic Instruments 


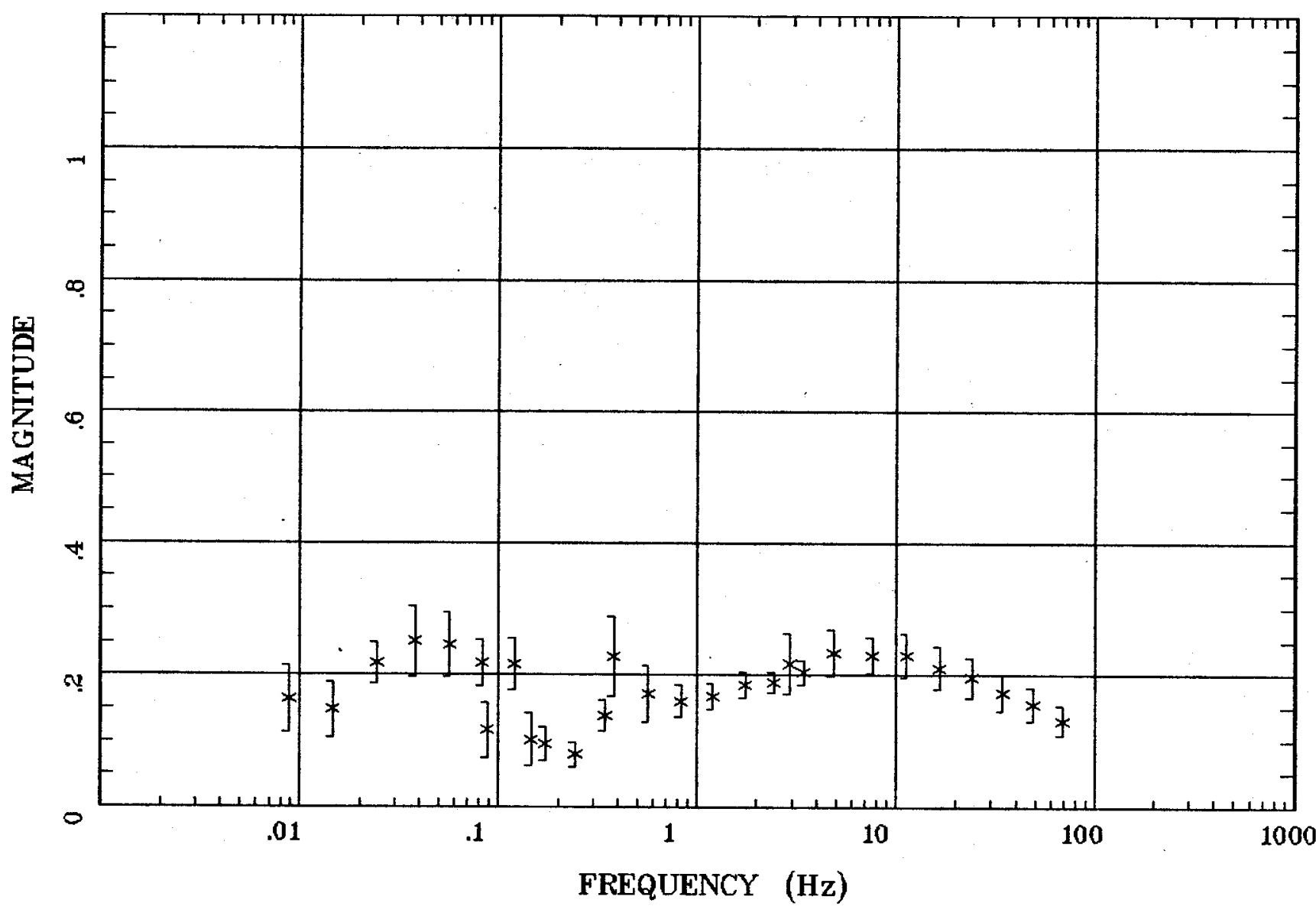

Client:

Remote: e-fld north $90 \mathrm{~m}$ Acquired: 13:4 Sep 06, 2003 Survey Co:USGS
Rotation:

Filename: ap06m.avg

Channels: Ch1 Ch2 Ch3 Ch4 Ch5 Ch6 Ch7 Plotted: 08:30 Sep 24, 2003

< EMI - ElectroMagnetic Instruments > 


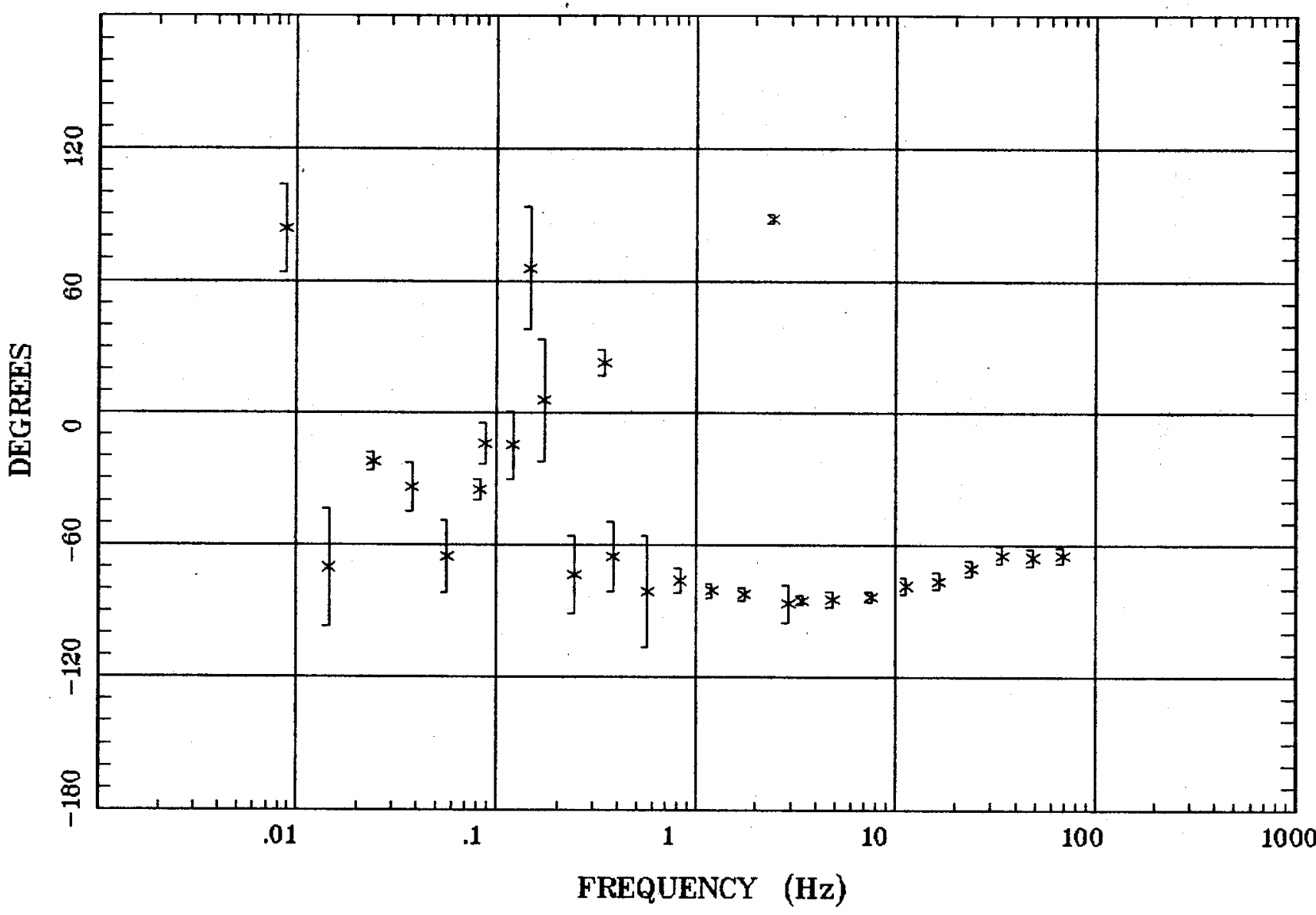

Client:

Remote: e-fld north $90 \mathrm{~m}$

Acquired: 13:4 Sep 06, 2003 Survey Co:USGS
Rotation:

Filename: ap06m.avg

Channels: Ch1 Ch2 Ch3 Ch4 Ch5 Ch6 Ch7

Plotted: 08:30 Sep 24, 2003

$<$ EMI - ElectroMagnetic Instruments > 


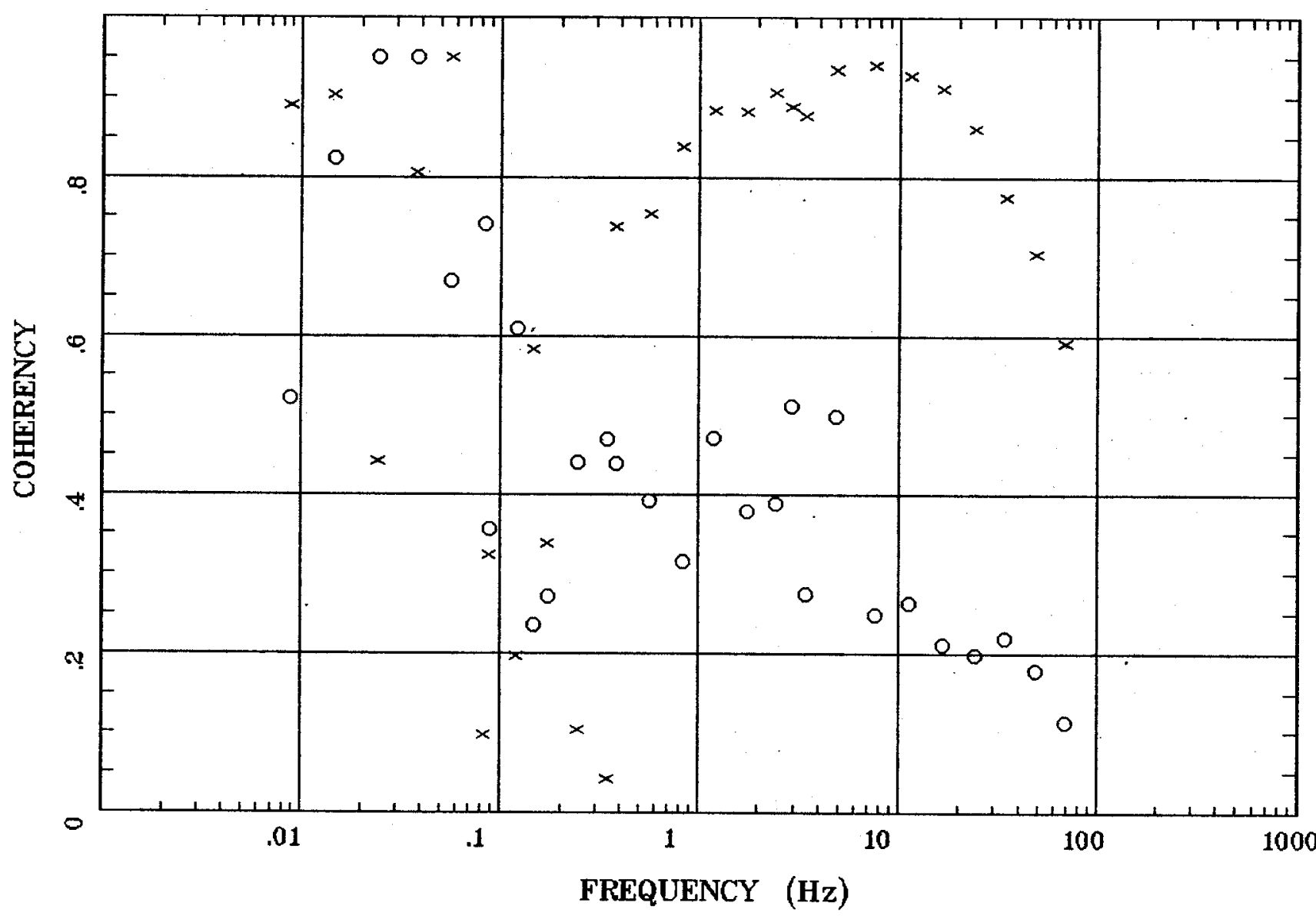

Client:

Remote: e-fld north $90 \mathrm{~m}$ Acquired: 13:4 Sep 06, 2003 Survey Co:USGS
Rotation:

Filename: ap $06 \mathrm{~m} . \mathrm{avg}$

Channels: Ch1 Ch2 Ch3 Ch4 Ch5 Ch6 Ch7 Plotted: 08:30 Sep 24, 2003

$<$ EMI - ElectroMagnetic Instruments 


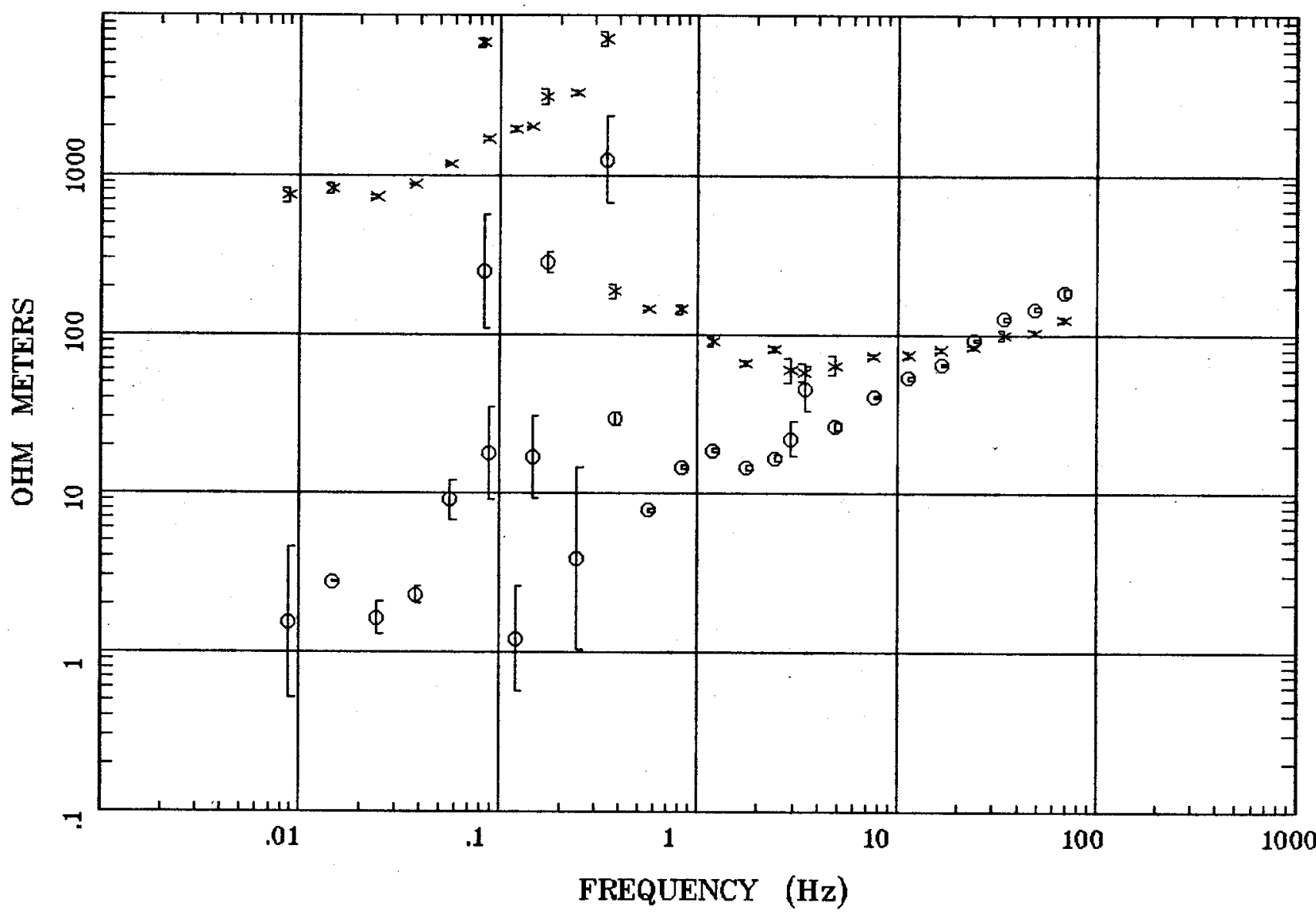

Client:

Remote: e-fld north $90 \mathrm{~m}$ Acquired: 10:5 Sep 07, 2003 Survey Co:USGS
Rotation:

Filename: ap07m.avg

Channels: Ch1 Ch2 Ch3 Ch4 Ch5 Ch6 Chr Plotted: 08:34 Sep 24, 2003

< EMI - ElectroMagnetic Instruments 


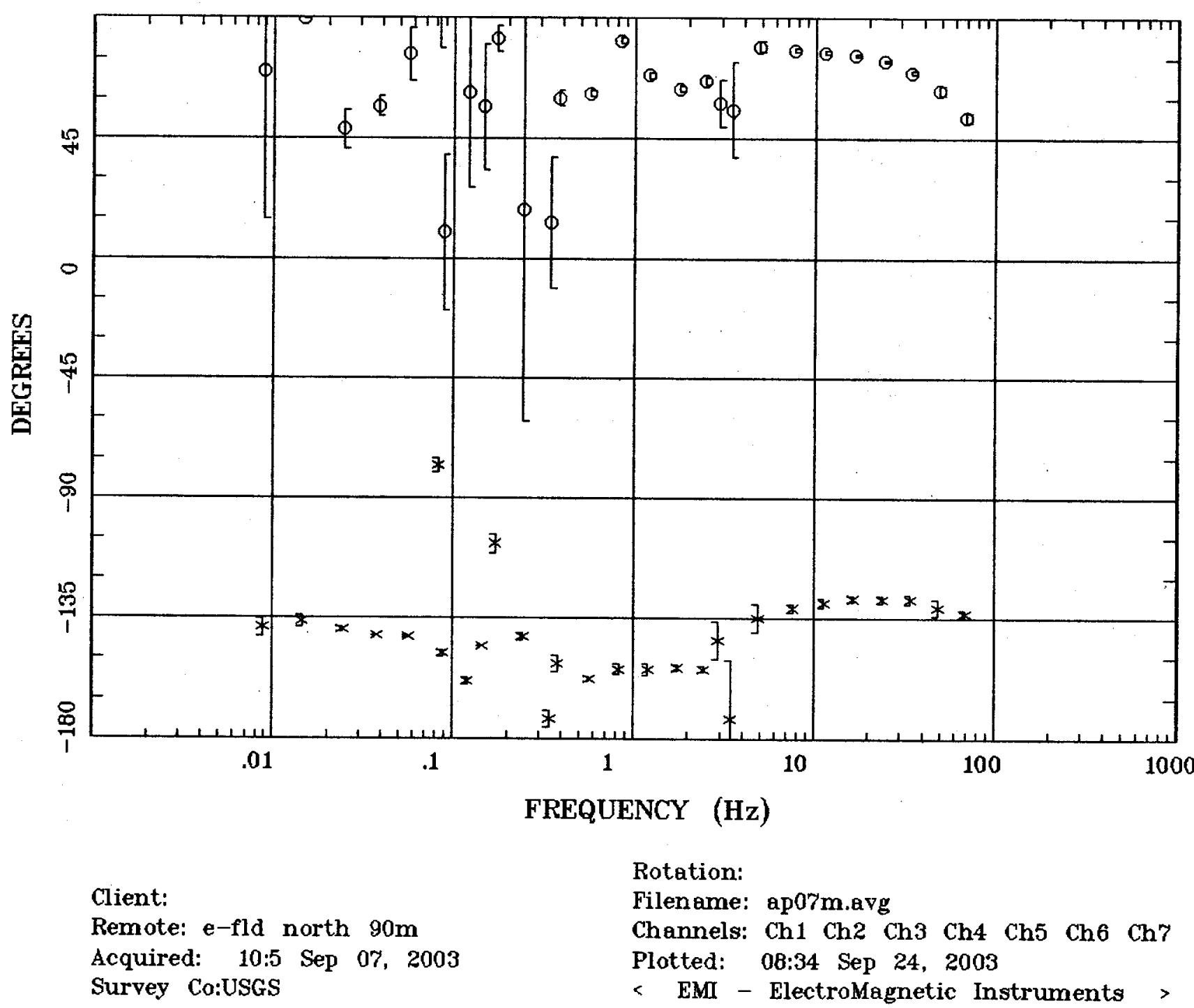




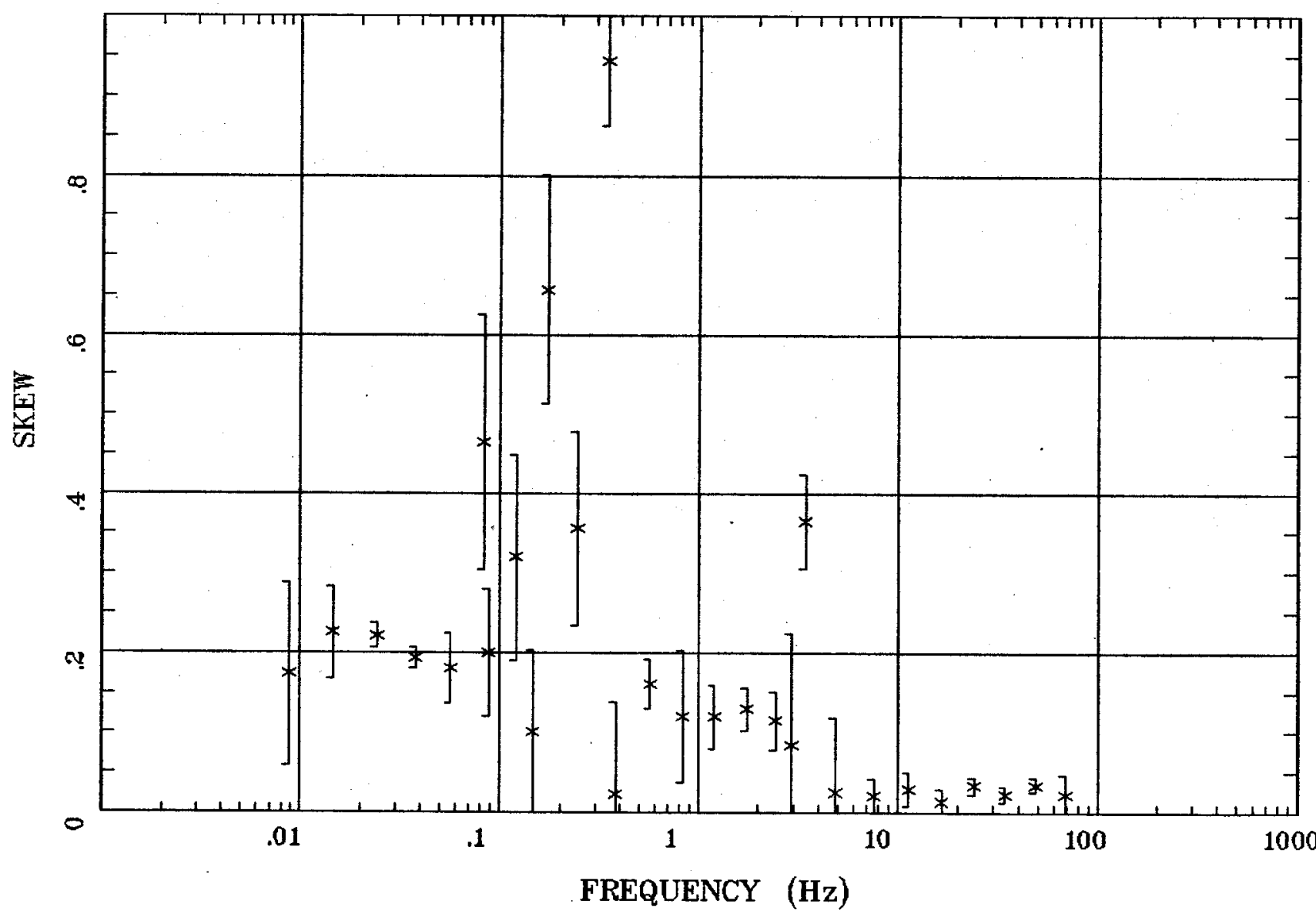

Client:

Remote: e-fld north $90 \mathrm{~m}$ Acquired: 10:5 Sep 07, 2003 Survey Co:USGS
Rotation:

Filename: ap07m.avg

Channels: Ch1 Ch2 Ch3 Ch4 Ch5 Ch6 Ch7 Plotted: 08:34 Sep 24, 2003

$<$ EMI - ElectroMagnetic Instruments 


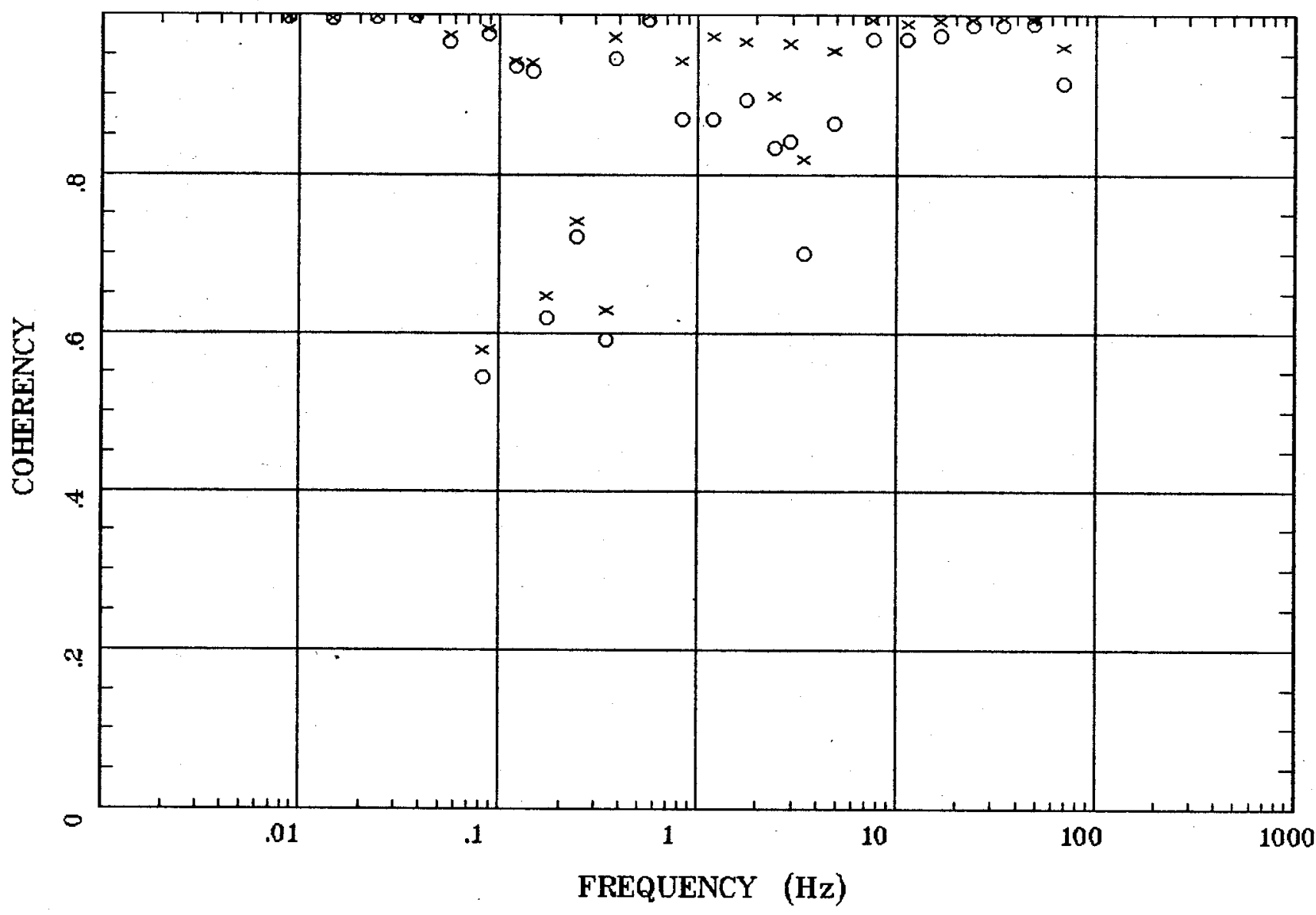

Client:

Remote: e-fld north $90 \mathrm{~m}$ Acquired: 10:5 Sep 07, 2003 Survey Co:USGS
Rotation:

Filename: ap0rim.avg

Channels: Ch1 Ch2 Ch3 Ch4 Ch5 Ch6 Ch7

Plotted: 08:34 Sep 24, 2003

$<$ EMI - ElectroMagnetic Instruments > 


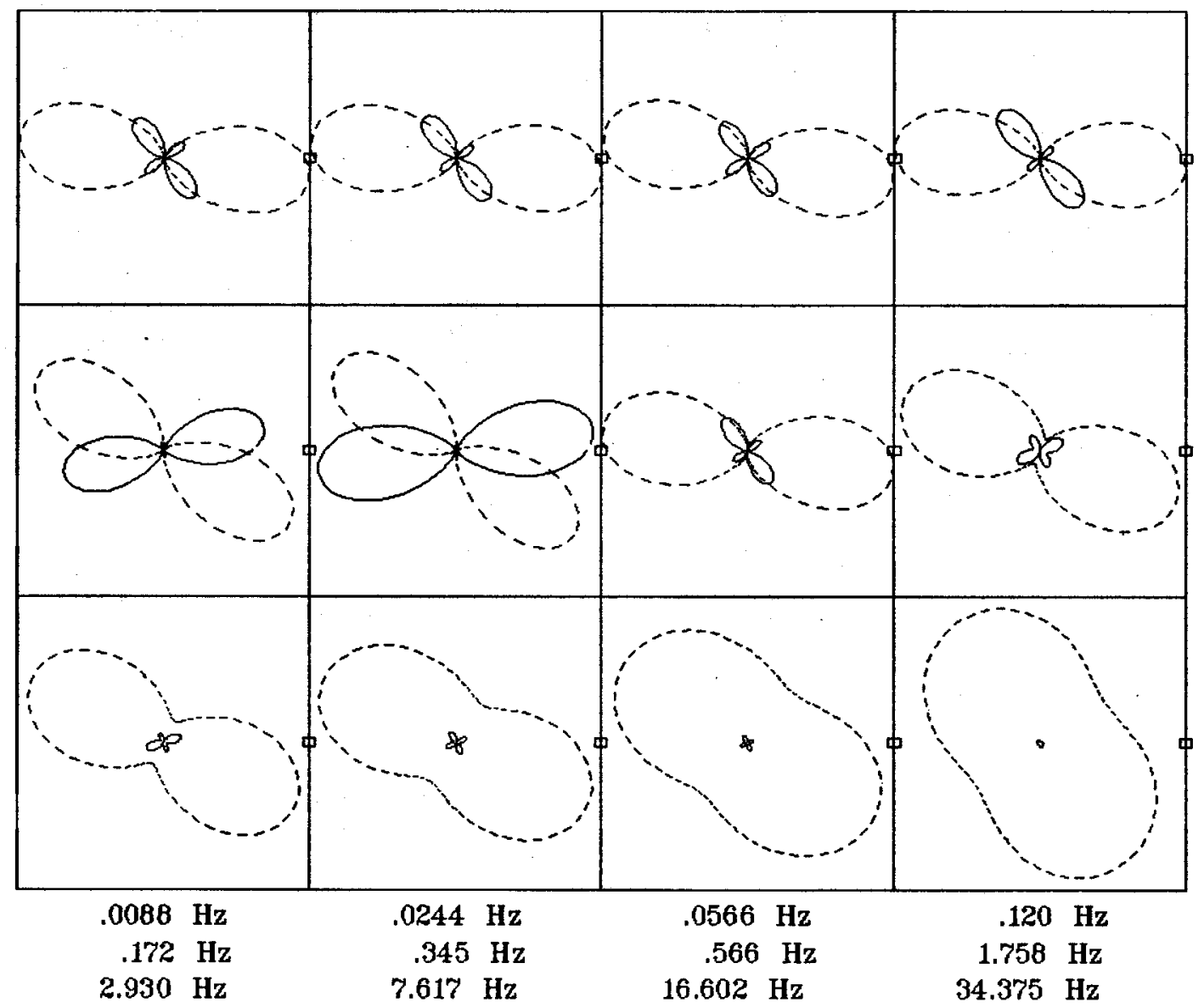

Client:

Remote: e-fld north $90 \mathrm{~m}$ Acquired: 10:5 Sep 07, 2003 Survey Co:USGS
Rotation:

Filename: ap07m.avg

Channels: Ch1 Ch2 Ch3 ch4 Ch5 Ch6 Ch7

Plotted: 08:34 Sep 24, 2003

< EMI - ElectroMagnetic Instruments > 


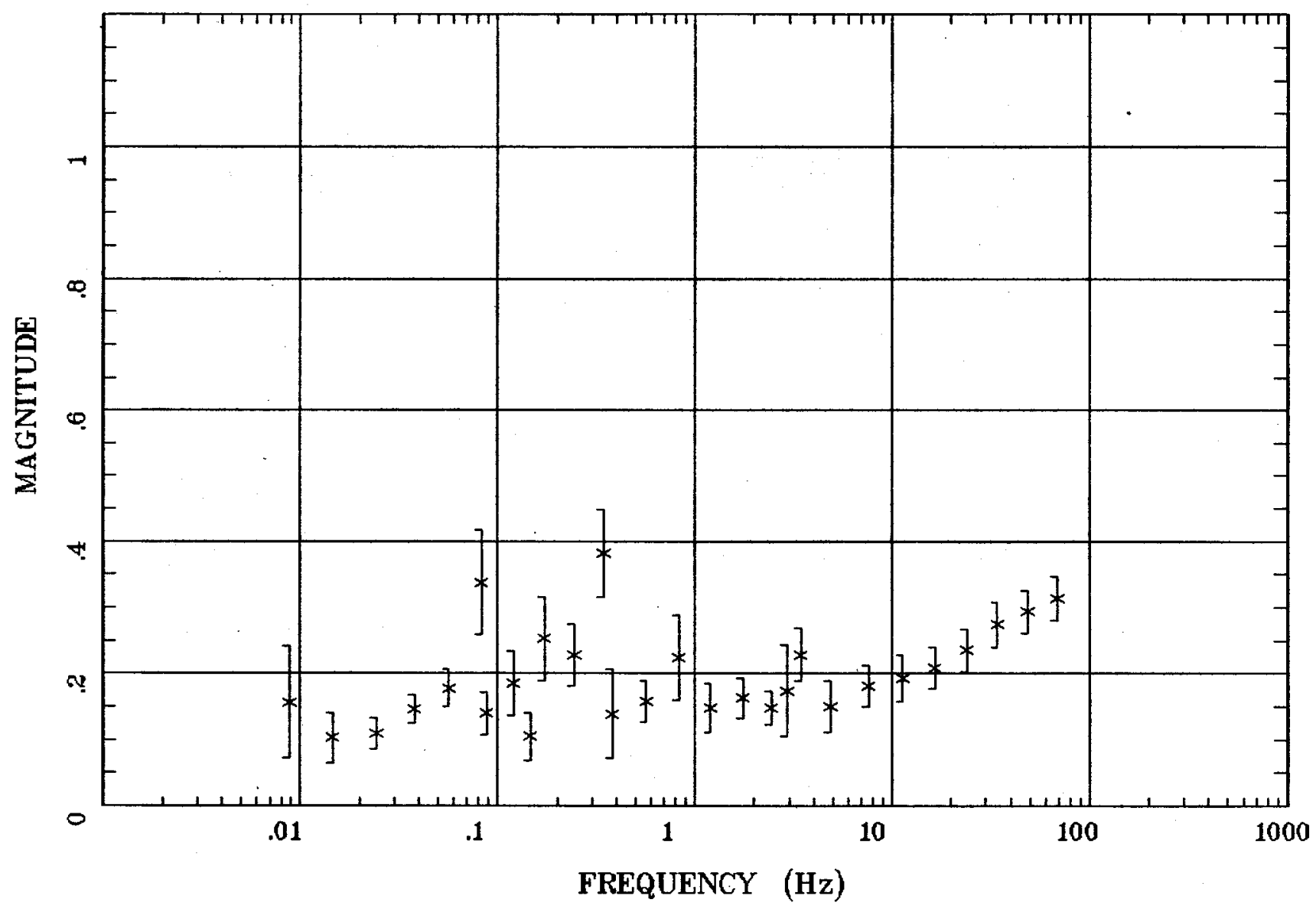

Client:

Remote: e-fld north $90 \mathrm{~m}$

Acquired: 10:5 Sep 07, 2003 Survey Co:USGS
Rotation:

Filename: ap07m.avg

Channels: Ch1 Ch2 Ch3 Ch4 Ch5 Ch6 Ch7 Plotted: 08:34 Sep 24, 2003

< EMI - ElectroMagnetic Instruments 
Station 7

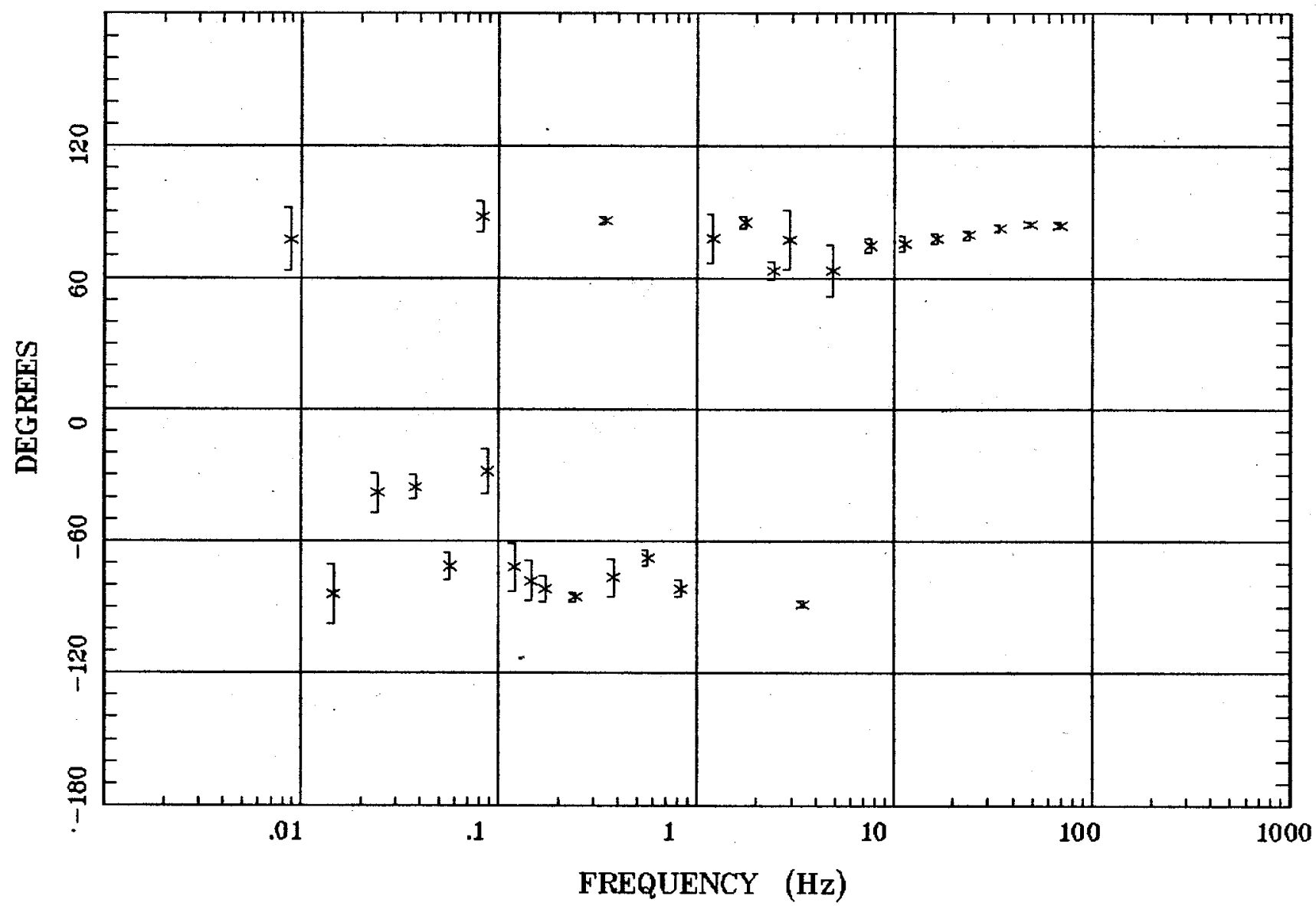

Client:

Remote: e-fld north $90 \mathrm{~m}$

Acquired: 10:5 Sep 07, 2003 Survey Co:USGS
Rotation:

Filename: ap07m.avg

Channels: Ch1 Ch2 Ch3 Ch4 Ch5 Ch6 Ch7 Plotted: 08:34 Sep 24, 2003

< EMI - ElectroMagnetic Instruments 


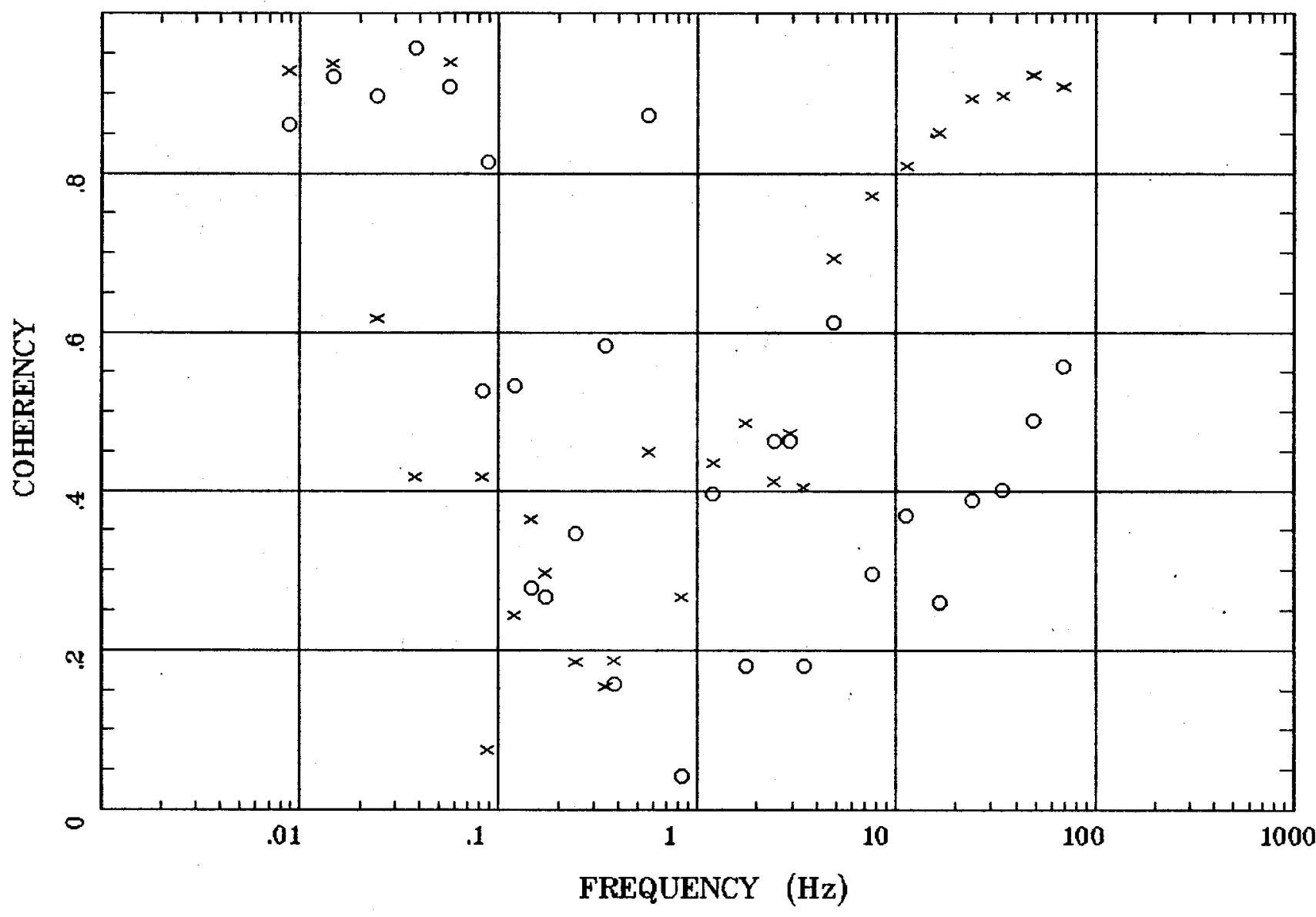

Client:

Remote: e-fld north $90 \mathrm{~m}$ Acquired: 10:5 Sep 07, 2003 Survey Co:USGS
Rotation:

Filename: ap0rm.avg

Channels: Ch1 Ch2 Ch3 Ch4 Ch5 Ch6 Ch7 Plotted: 08:34 Sep 24, 2003

< EMI - ElectroMagnetic Instruments 


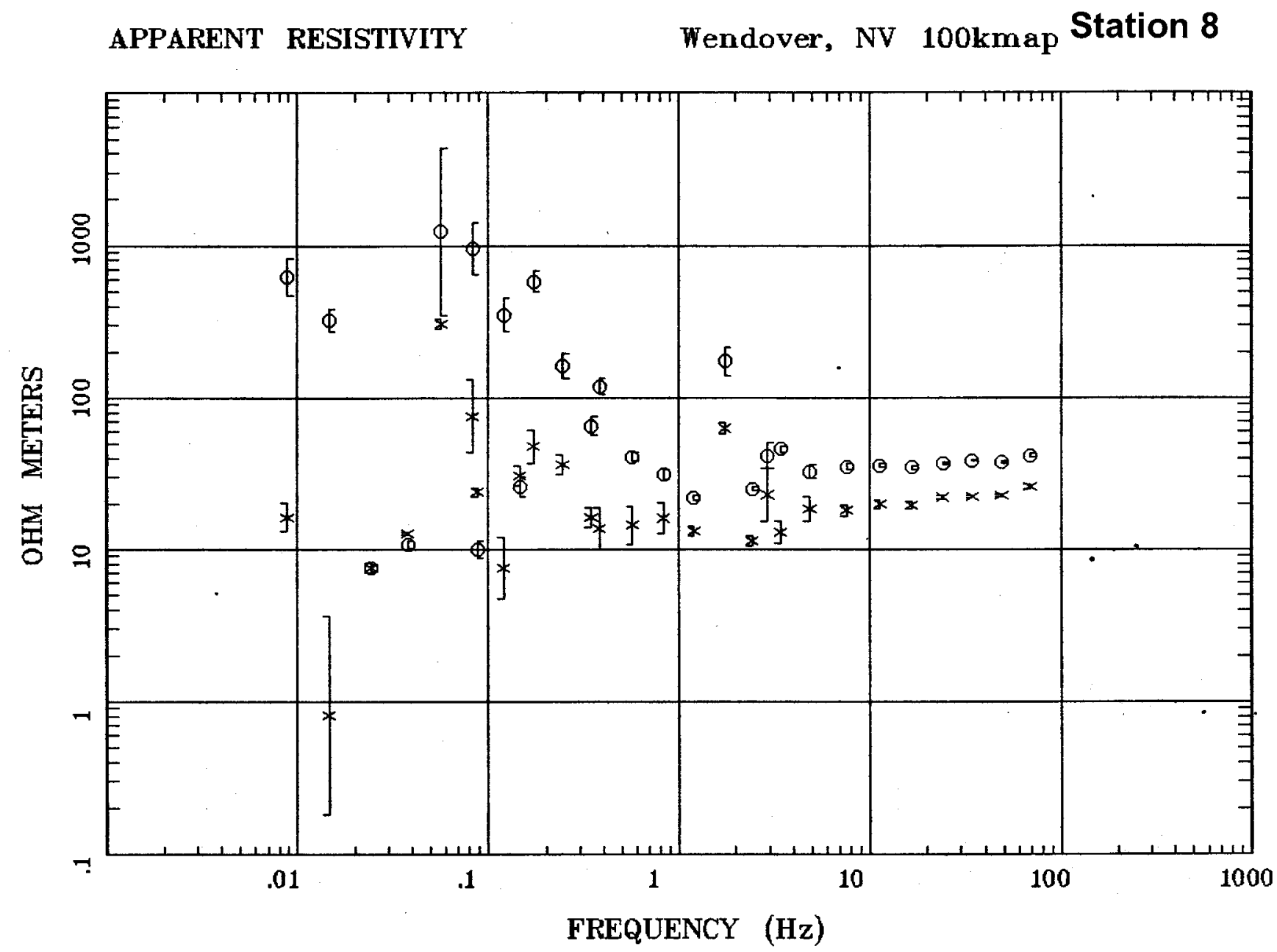

Client:

Remote: e-fld north $90 \mathrm{~m}$ Acquired: 15:5 Sep 07, 2003 Survey Co:USGS
Rotation:

Filename: ap08n.avg

Channels: Ch6 Ch7 Ch3 Ch4 Ch5 Ch6 Ch7

Plotted: 14:26 Sep 24, 2003

< EMI - ElectroMagnetic Instruments 


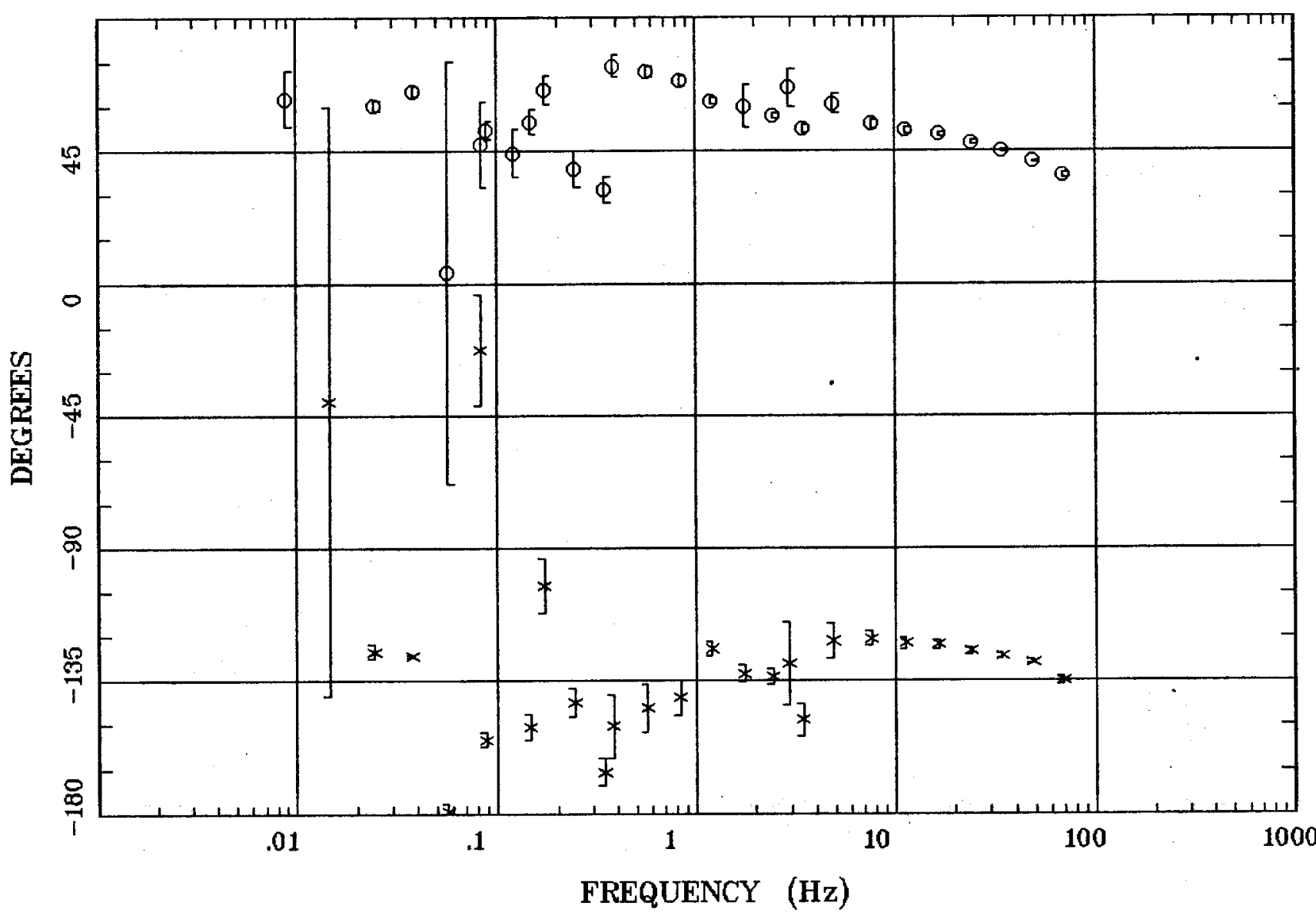

Client:

Remote: e-fld north $90 \mathrm{~m}$

Acquired: 15:5 Sep 07, 2003

Survey Co:USGS
Rotation:

Filename: ap08n.avg

Channels: Ch6 Ch7 Ch3 Ch4 Ch5 Ch6 Ch7

Plotted: 14:27 Sep 24, 2003

< EMI - ElectroMagnetic Instruments 


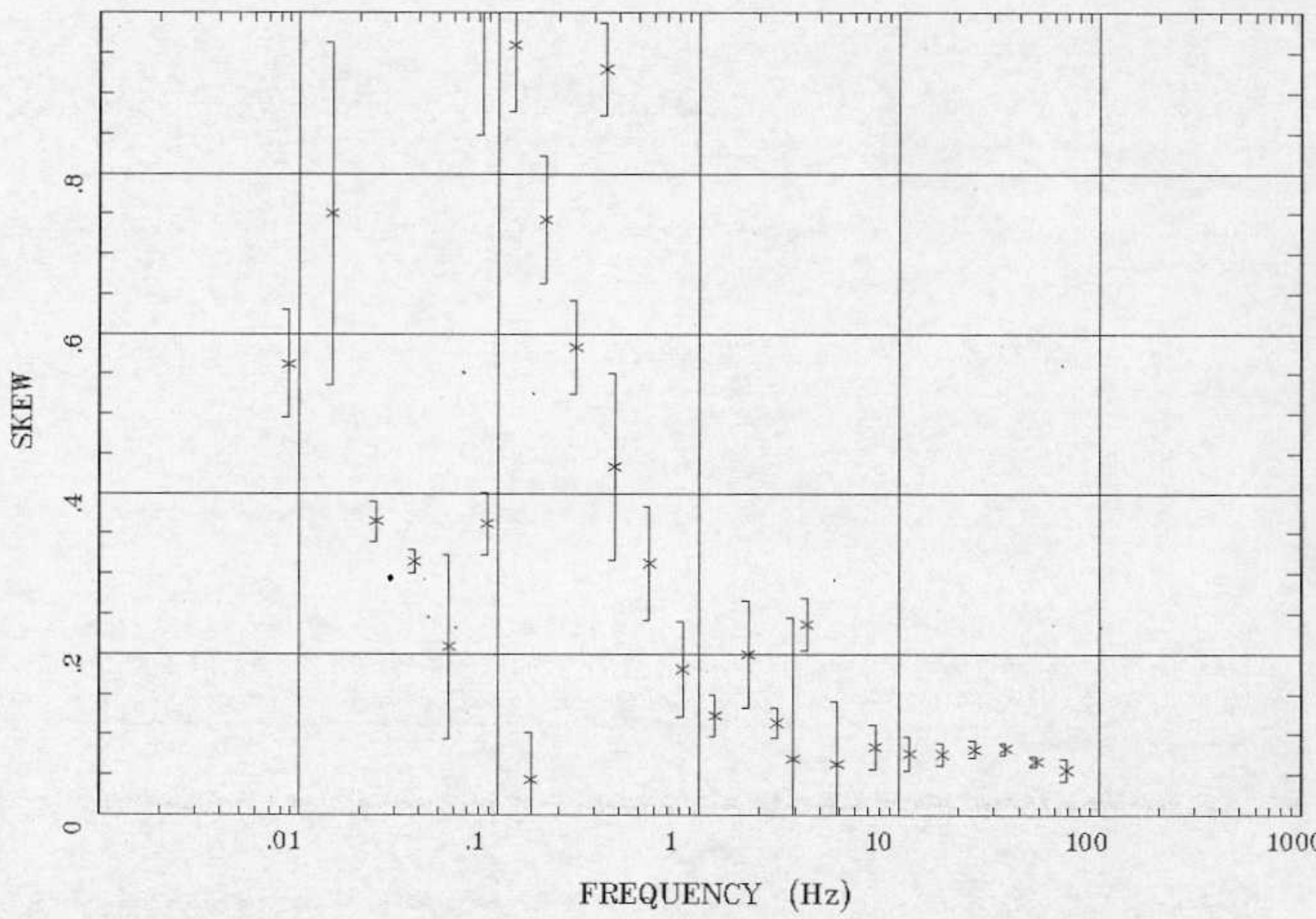

Client:

Remote: e-fld north $90 \mathrm{~m}$ Acquired: 15:5 Sep 07, 2003 Survey Ca:USGS
Rotation:

Filename: ap08n.avg

Channels: Ch6 Ch7 Ch3 Ch4 Ch5 Ch6 Ch7 Plotted: 14:27 Sep 24, 2003

$<$ EMI - ElectroMagnetic Instruments 
Wendover, NV 100kmap Station 8

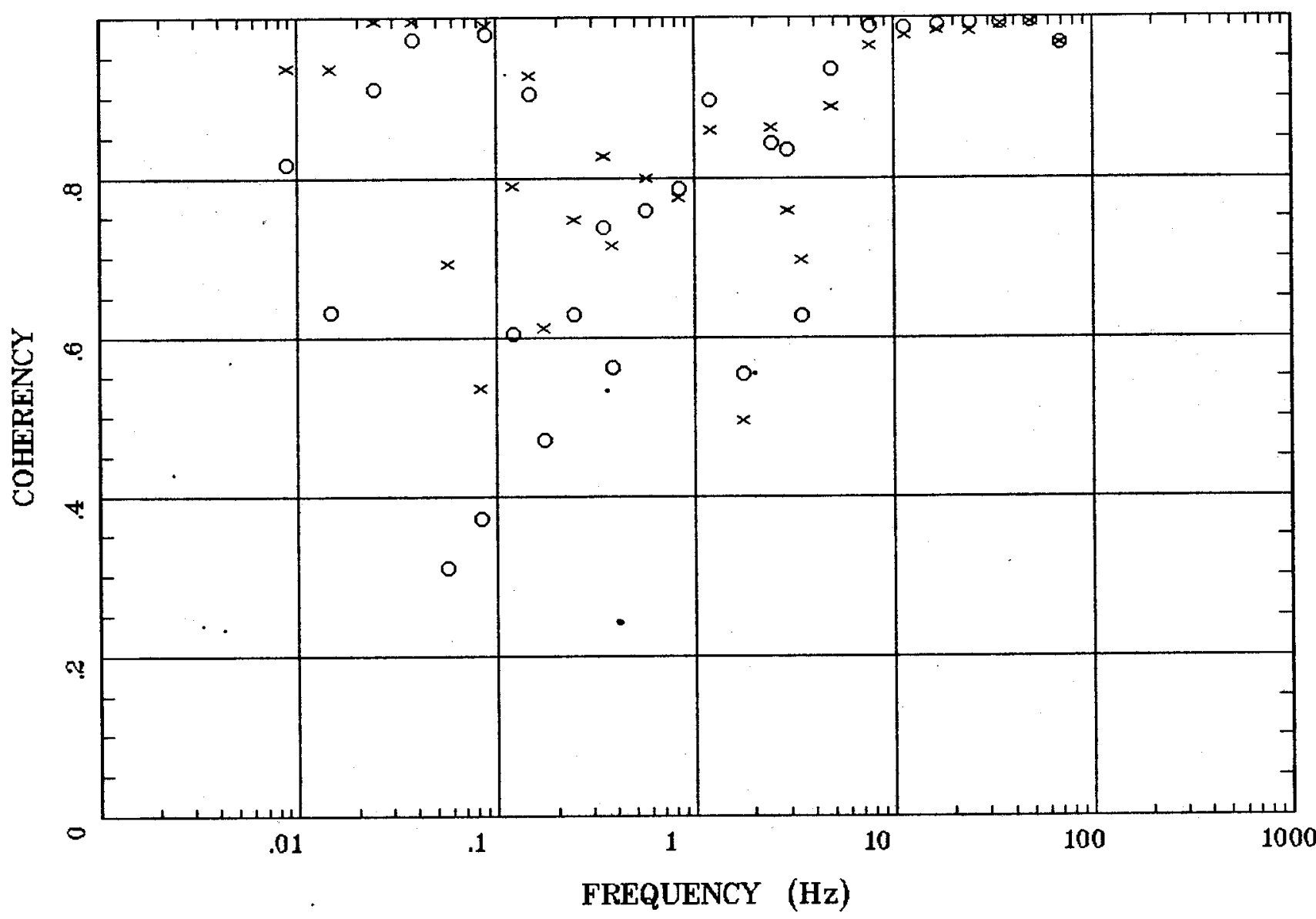

Client:

Remote: e-fld north $90 \mathrm{~m}$ Acquired: 15:5 Sep 07, 2003 Survey Co:USGS
Rotation:

Filename: ap08n.avg

Channels: Ch6 Ch7 Ch3 Ch4 Ch5 Ch6 Ch7 Plotted: 14:27 Sep 24, 2003

< EMI - ElectroMagnetic Instruments > 


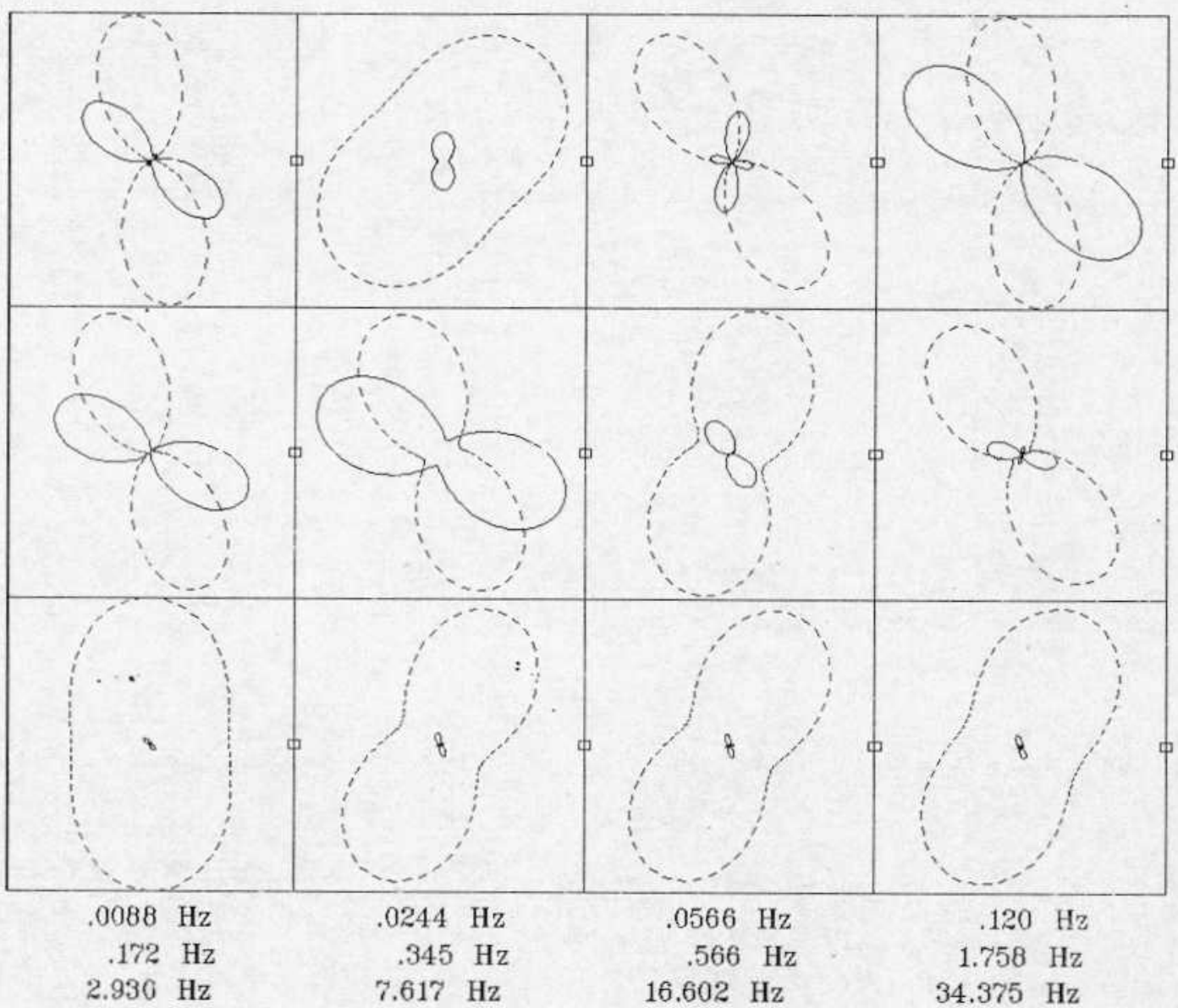

Client:

Remote: e-fld north $90 \mathrm{~m}$ Acquired: 15:5 Sep 07, 2003 Survey Co:USGS
Rotation:

Filename: ap08n.avg

Channels: Ch6 Ch 7 Ch3 Ch4 Ch5 Ch6 Ch7

Plotted: 14:27 Sep 24, 2003

$<$ EMI - ElectroMagnetic Instruments 


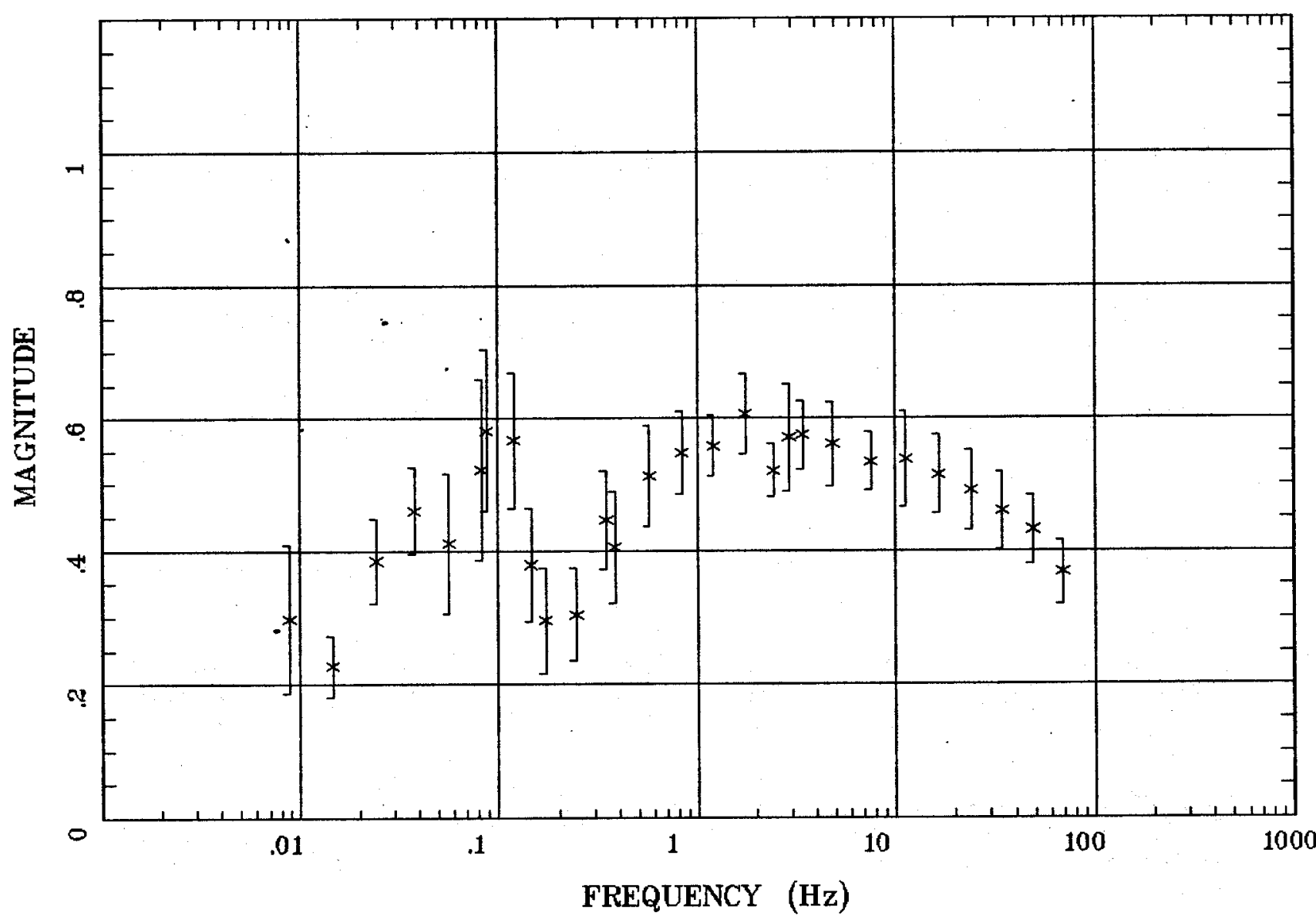

Client:

Remote: e-fld north $90 \mathrm{~m}$

Acquired: 15:5 Sep 07, 2003

Survey Co:USGS
Rotation:

Filename: ap08n.avg

Channels: Ch6 Ch7 Ch3 Ch4 Ch5 Ch6 Ch7

Plotted: 14:27 Sep 24, 2003

< EMI - ElectroMagnetic Instruments > 


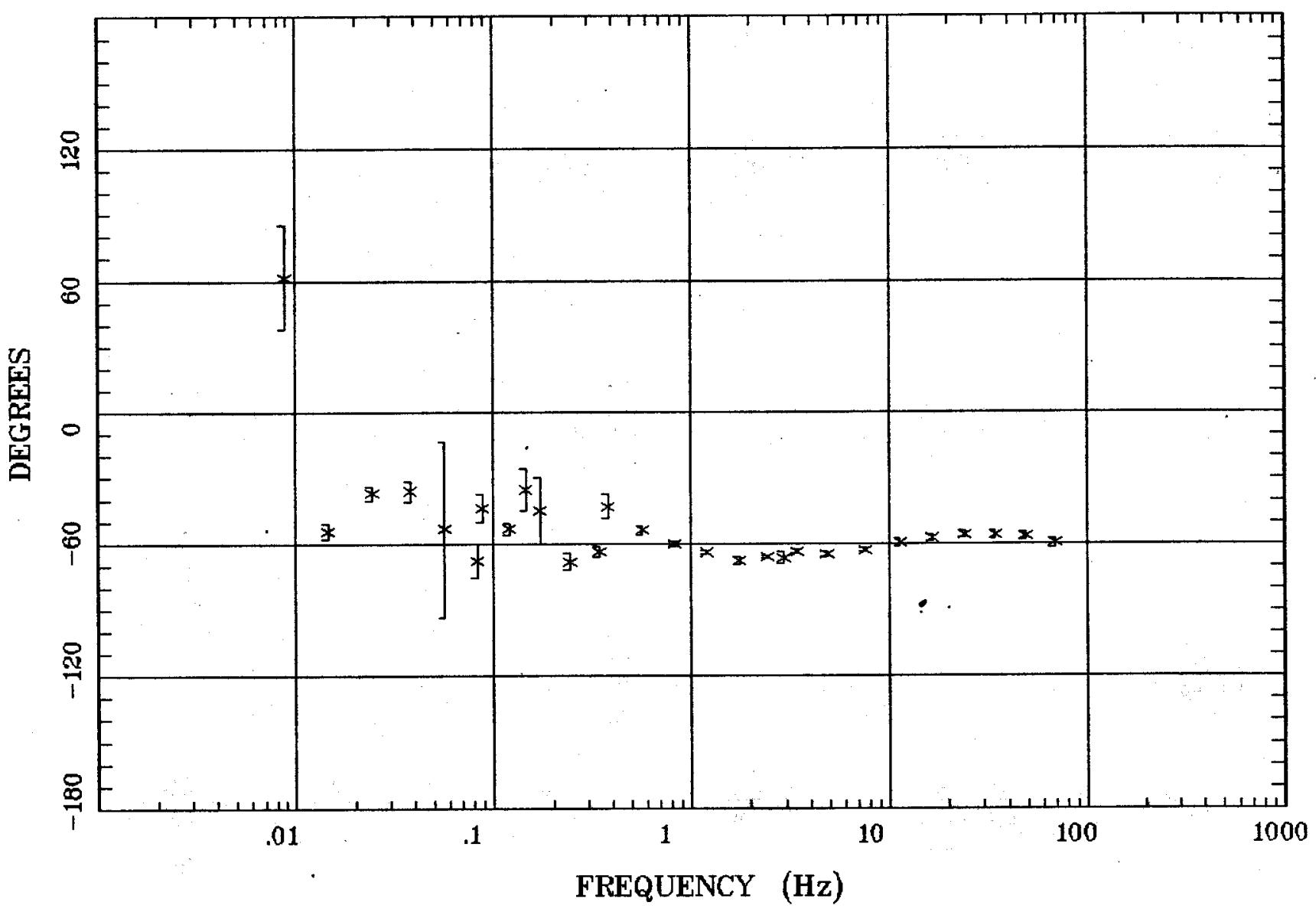

Client:

Remote: e-fld north $90 \mathrm{~m}$ Acquired: 15:5 Sep 07, 2003 Survey Co:USGS
Rotation:

Filename: ap08n.avg

Channels: Ch6 Ch7 Ch3 Ch4 Ch5 Ch6 Ch7

Plotted: 14:27 Sep 24, 2003

$<$ EMI - ElectroMagnetic Instruments 
Wendover, NV 100kmap Station 8

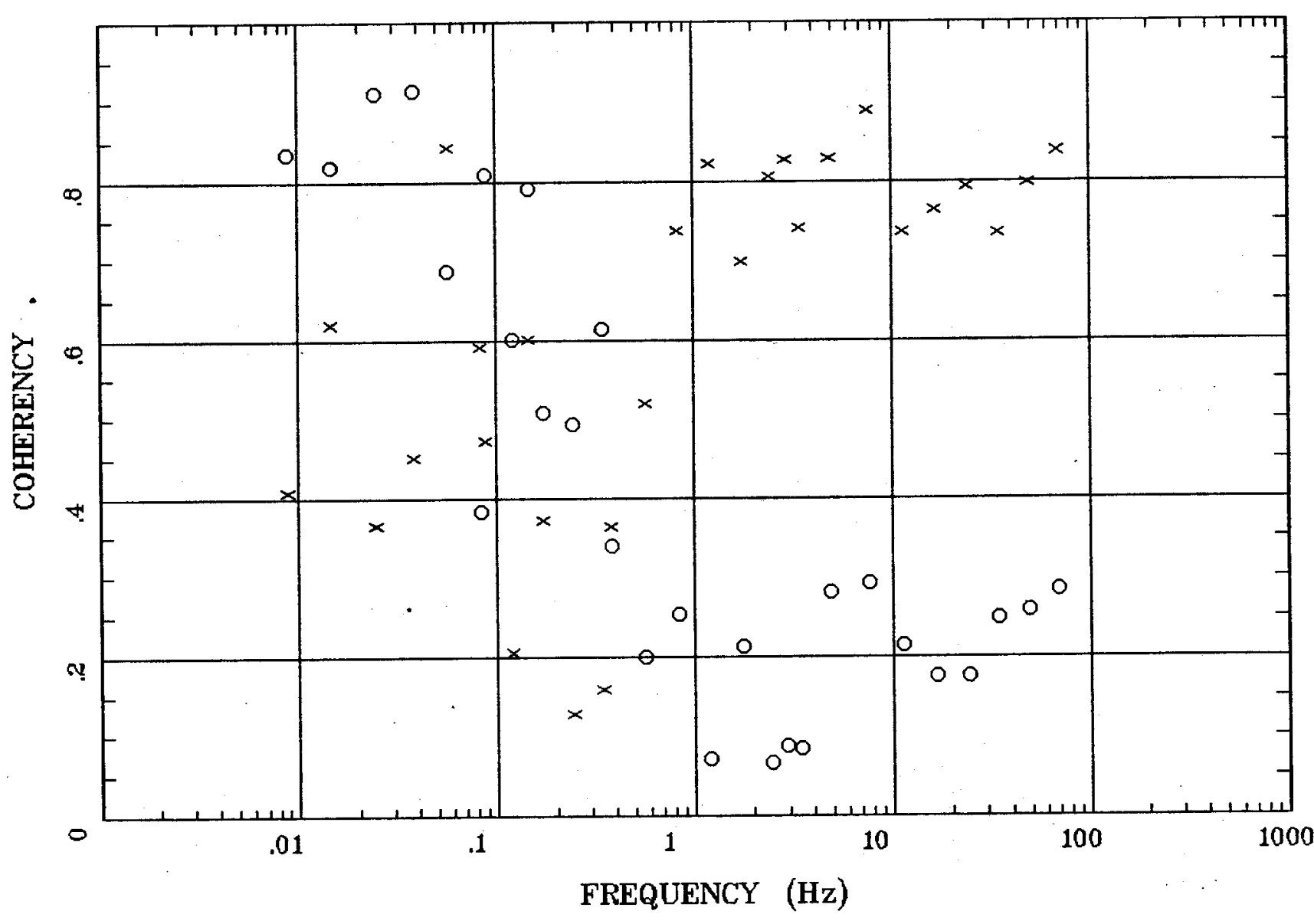

Client:

Remote: e-fld north $90 \mathrm{~m}$ Acquired: 15:5 Sep 07, 2003 Survey Co:USGS
Rotation:

Filename: ap08n.avg

Channels: $\mathrm{Ch} 6 \mathrm{Ch} 7 \mathrm{Ch} 3 \mathrm{Ch} 4 \mathrm{Ch} 5 \mathrm{Ch} 6 \mathrm{Ch} 7$ Plotted: 14:27 Sep 24, 2003

$<$ EMI - ElectroMagnetic Instruments 


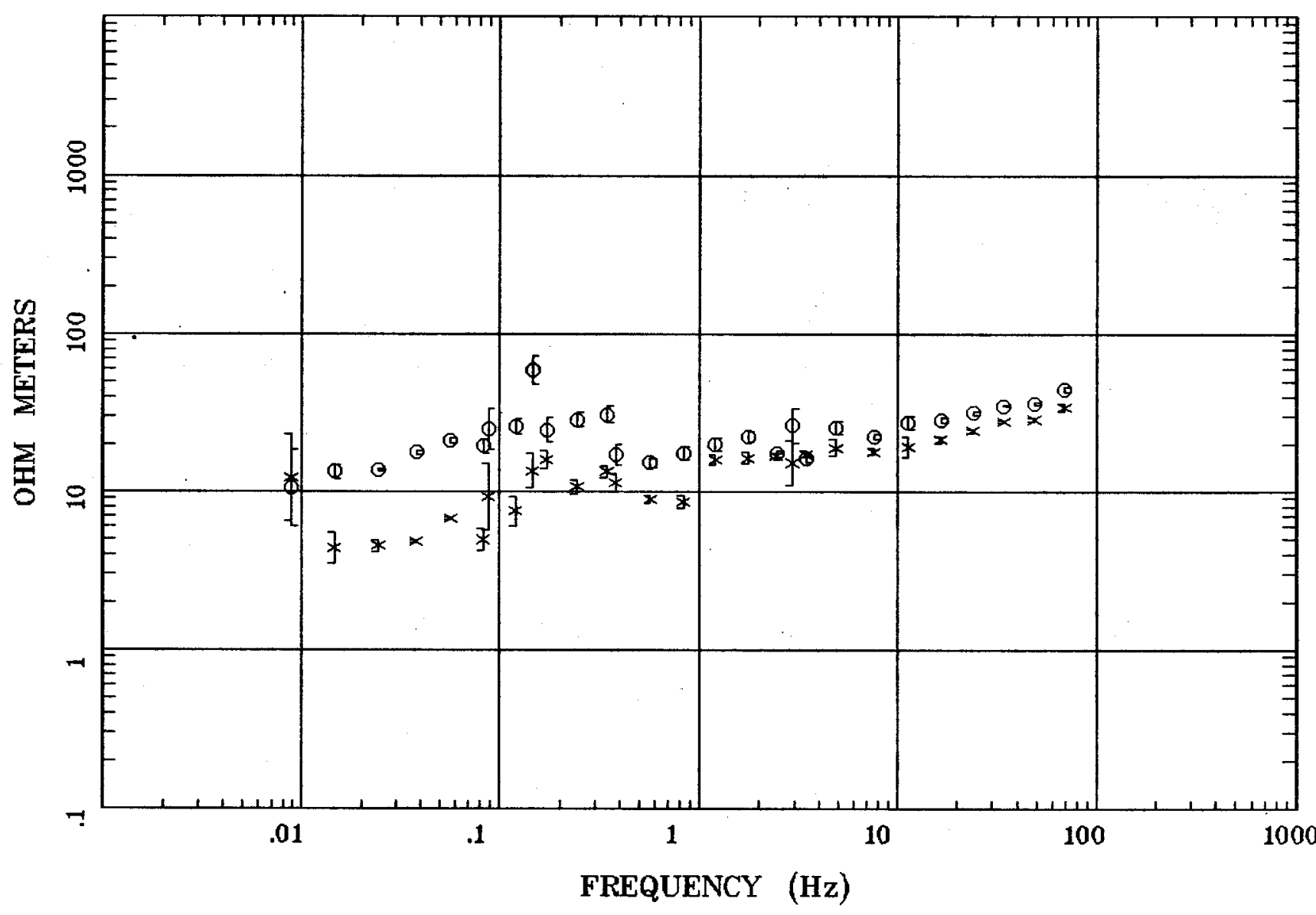

Client:

Remote: e-fld south $90 \mathrm{~m}$ Acquired: 10:0 Sep 08, 2003 Survey Co:USGS

\section{Rotation:}

Filename: ap09m.avg

Channels: Ch1 Ch2 Ch3 Ch4 Ch5 Ch6 Ch7 Plotted: 08:36 Sep 24, 2003

$<$ EMI - ElectroMagnetic Instruments 


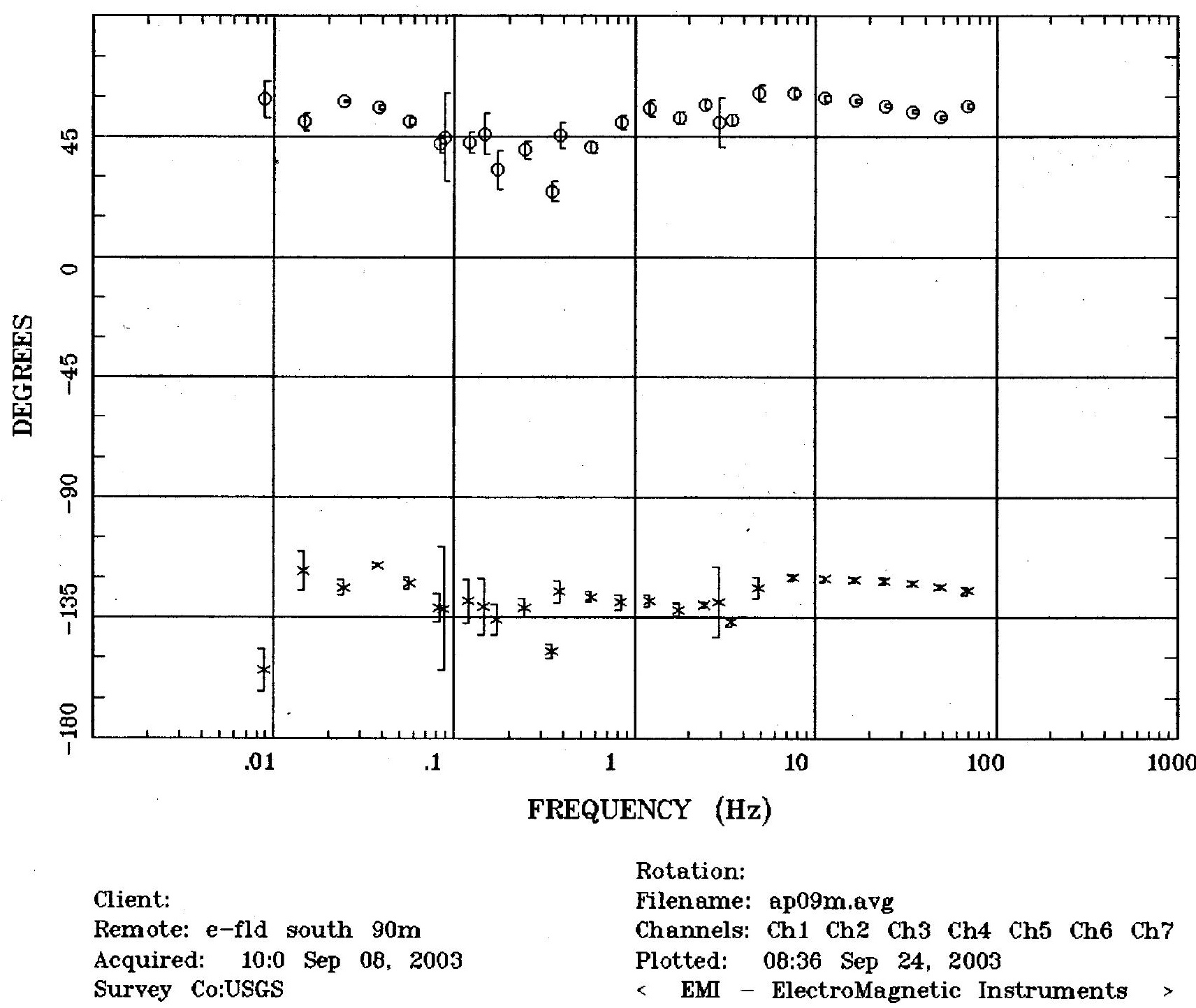




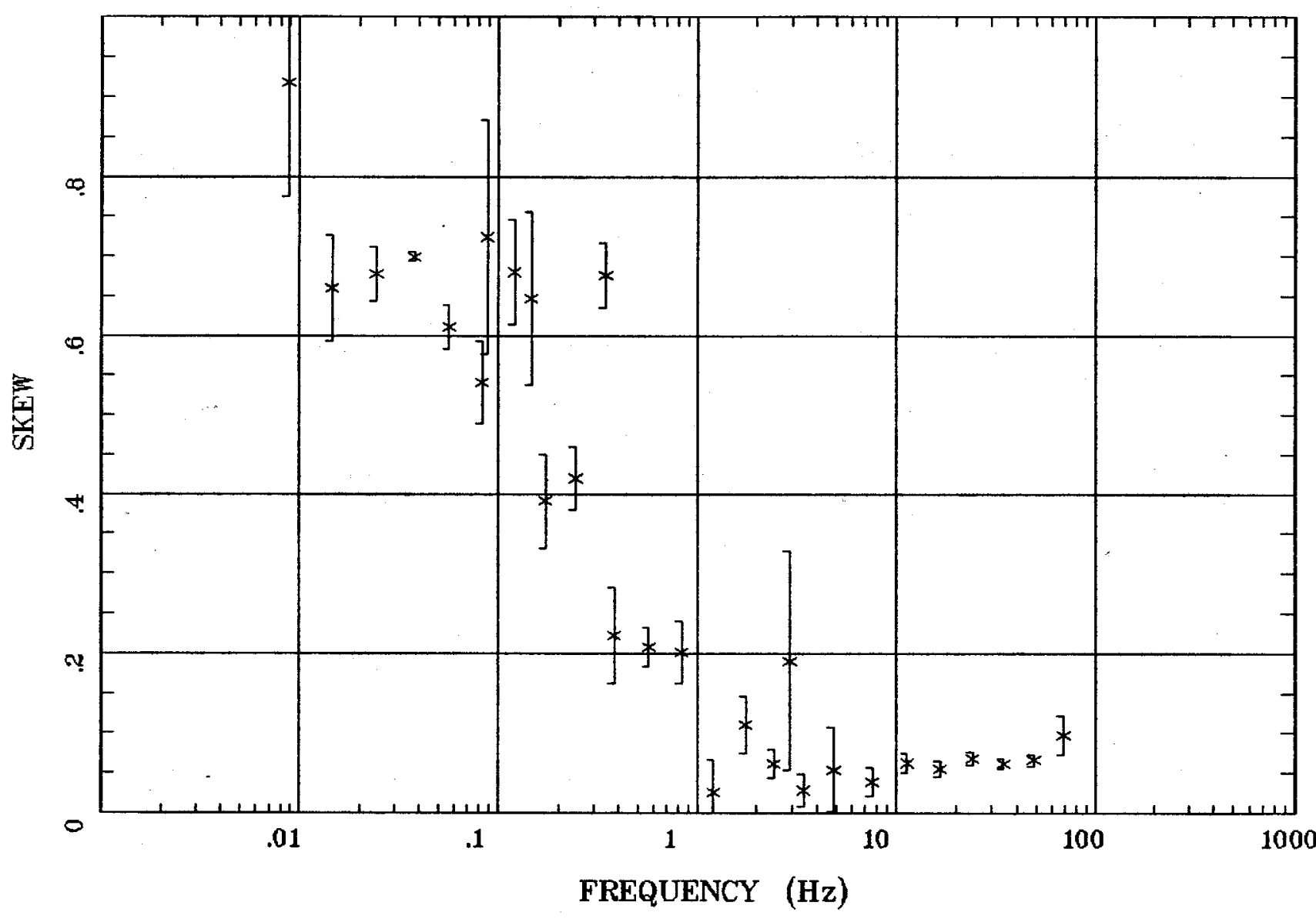

Client:

Remote: e-fld south $90 \mathrm{~m}$ Acquired: 10:0 Sep 08, 2003 Survey Co:USGS
Rotation:

Filename: ap09m.avg

Channels: Ch1 Ch2 Ch3 Ch4 Ch5 Ch6 Ch7 Plotted: 08:36 Sep 24, 2003

$<$ EMI - ElectroMagnetic Instruments > 


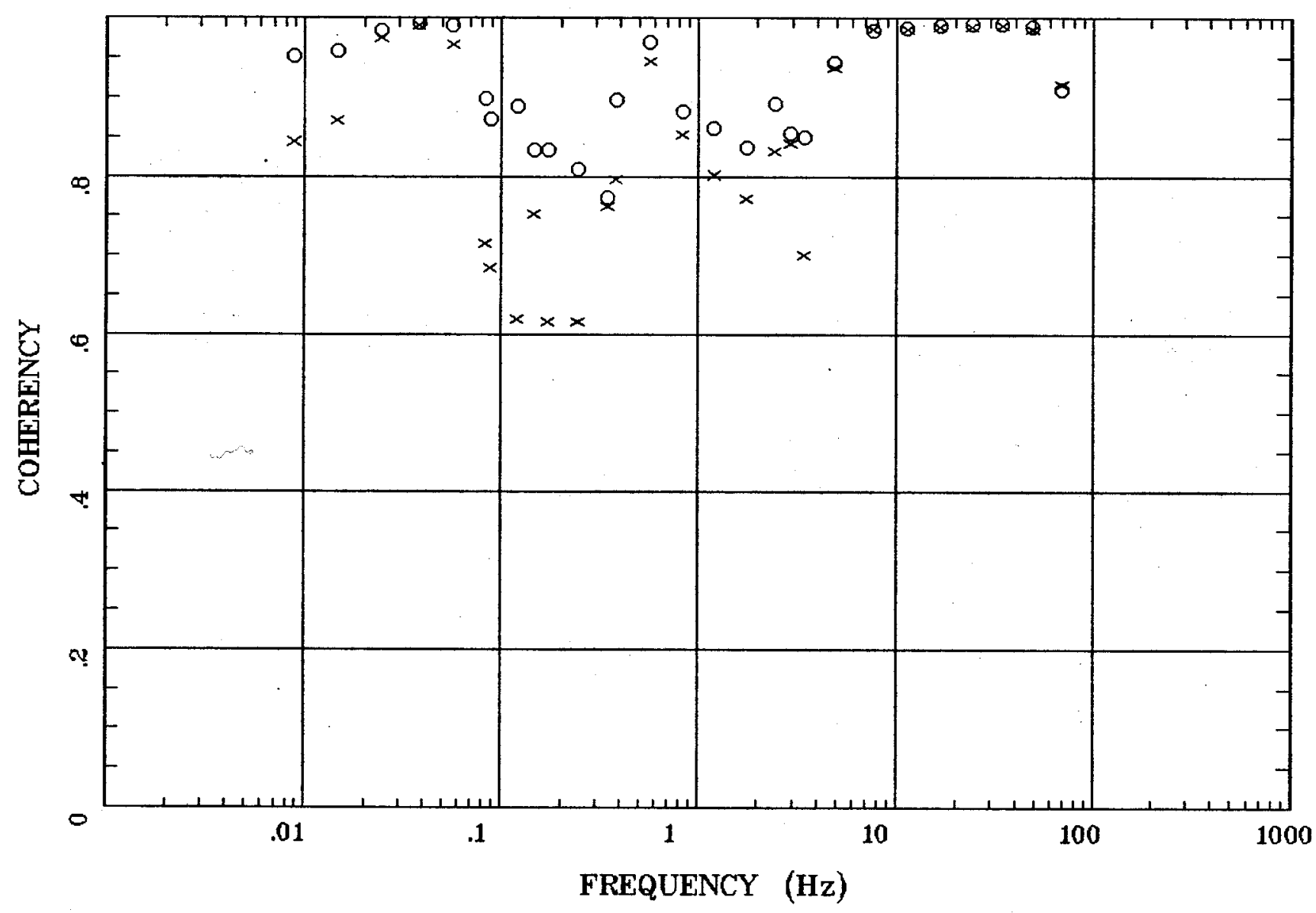

Client:

Remote: e-fld south $90 \mathrm{~m}$

Acquired: 10:0 Sep 08, 2003

Survey Co:USGS
Rotation:

Filenome: ap09m.avg

Channels: Ch1 Ch2 Ch3 Ch4 Ch5 Ch6 Ch7

Plotted: 08:36 Sep 24, 2003

< EMI - ElectroMagnetic Instruments 


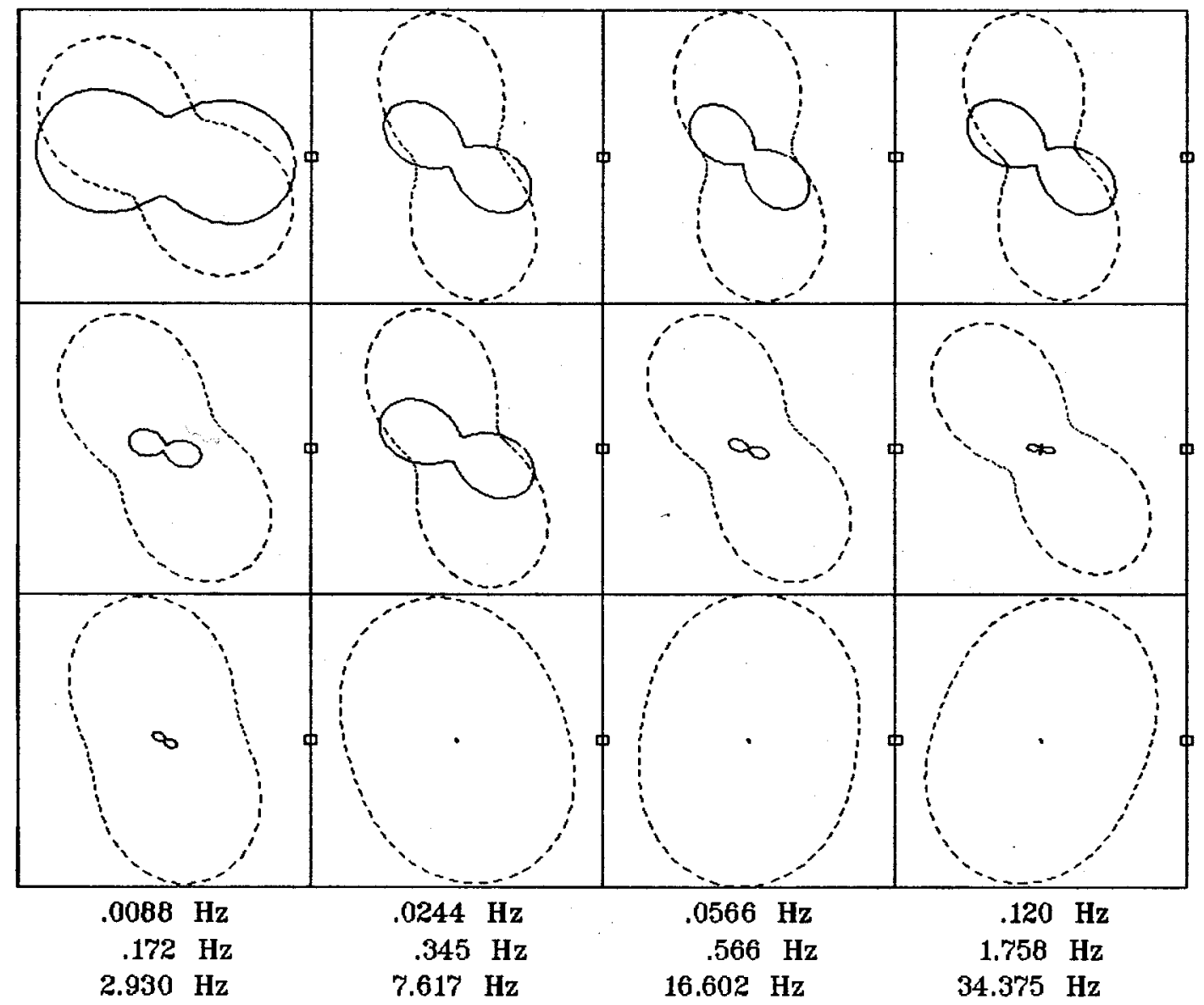

\section{Client:}

Remote: e-fld south $90 \mathrm{~m}$ Acquired: 10:0 Sep 08, 2003 Survey Co:USGS
Rotation:

Filename: ap09m.avg

Channels: Ch1 Ch2 Ch3 Ch4 Ch5 Ch6 Ch7

Plotted: 08:36 Sep 24, 2003

< EMI - ElectroMagnetic Instruments 


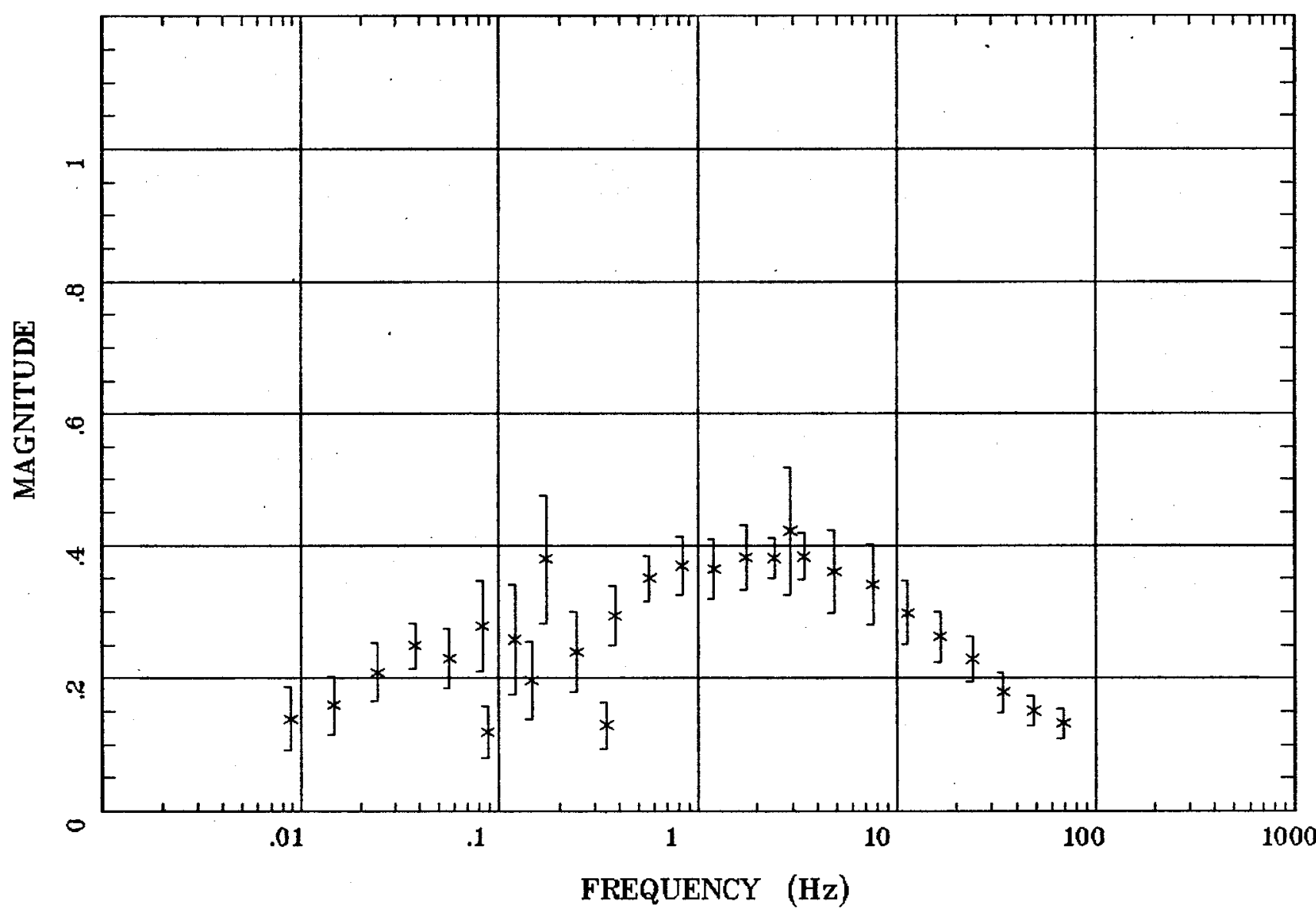

Client:

Remote: e-fld south $90 \mathrm{~m}$ Acquired: 10:0 Sep 08, 2003 Survey Co:USGS
Rotation:

Filename: ap09m.avg

Channels: Ch1 Ch2 Ch3 ch4 Ch5 Ch6 Ch7 Plotted: 08:36 Sep 24, 2003

$<$ EMI - ElectroMagnetic Instruments 


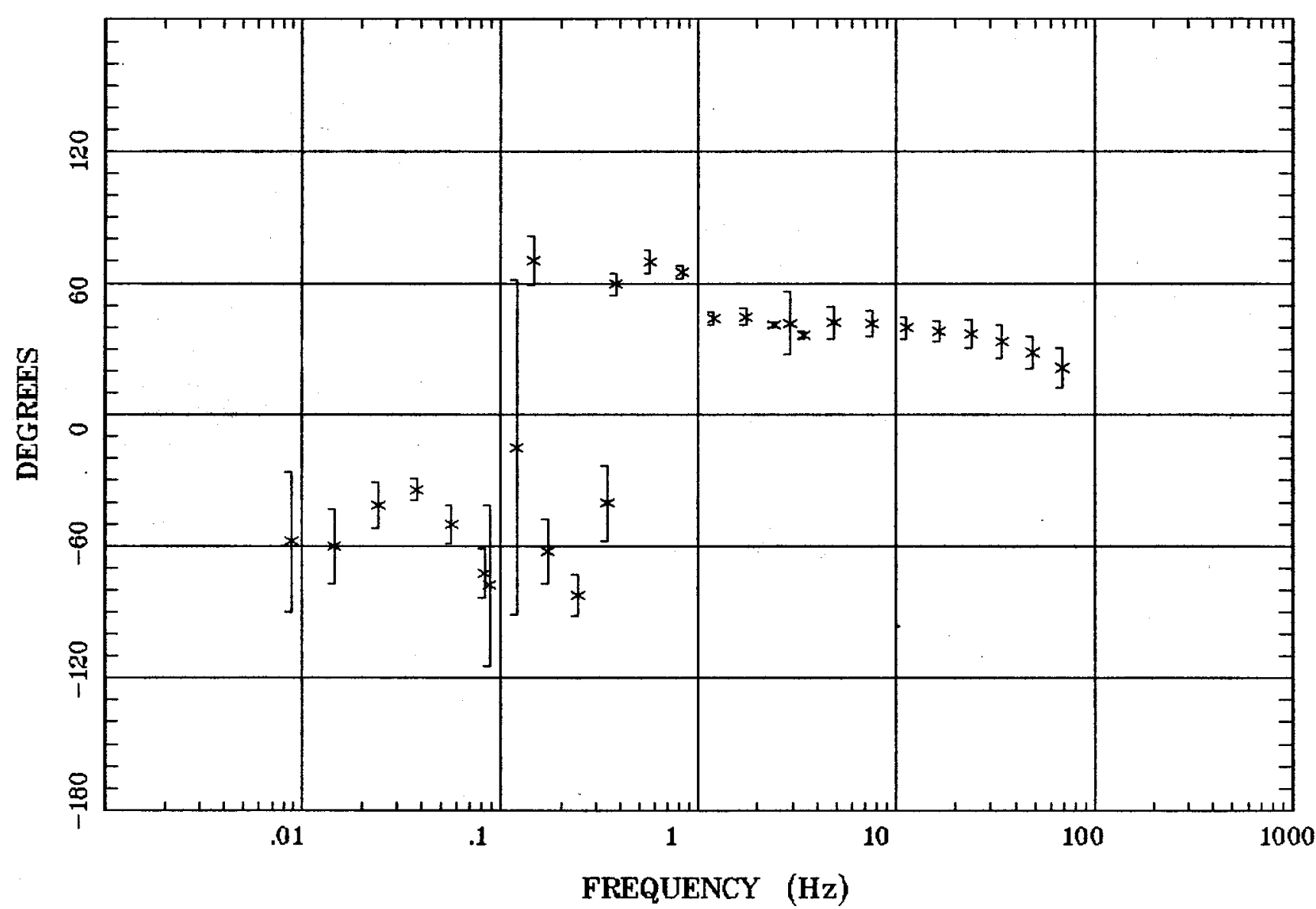

Client:

Remote: e-fld south $90 \mathrm{~m}$ Acquired: 10:0 Sep 08, 2003 Survey Co:USGS
Rotation:

Filename: ap09m.avg

Channels: Ch1 Ch2 Ch3 Ch4 Ch5 Ch6 Ch7 Plotted: 08:36 Sep 24, 2003

< EMI - ElectroMagnetic Instruments 
HzHx.x Coh HzHy.o

Wendover, NV 100kmap Station 9

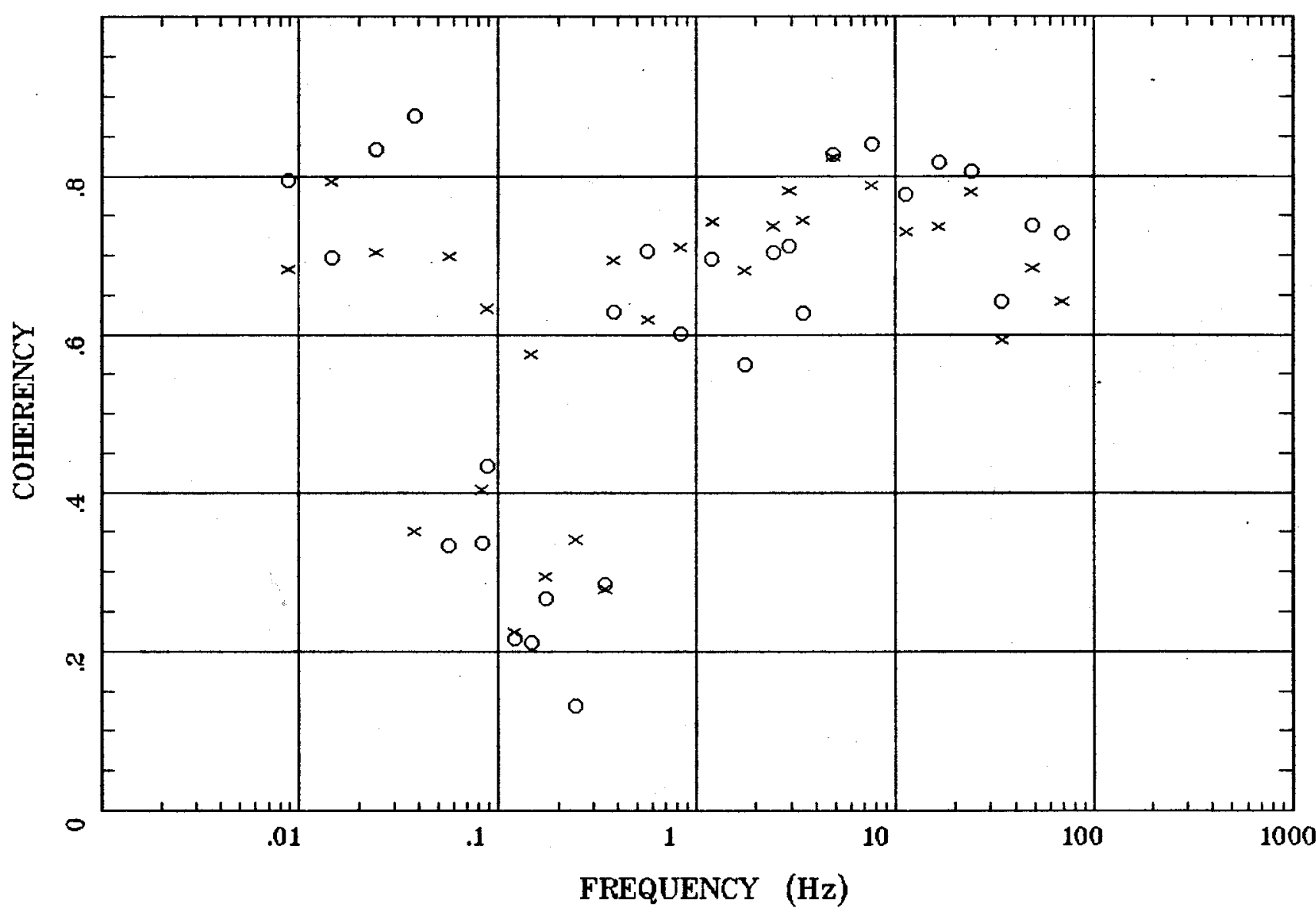

Client:

Remote: e-fld south $90 \mathrm{~m}$ Acquired: 10:0 Sep 08, 2003 Survey Co:USGS
Rotation:

Filename: ap09m.avg

Channels: Ch1 Ch2 Ch3 Ch4 Ch5 Ch6 Ch7

Plotted: 08:36 Sep 24, 2003

< EMI - ElectroMagnetic Instruments 


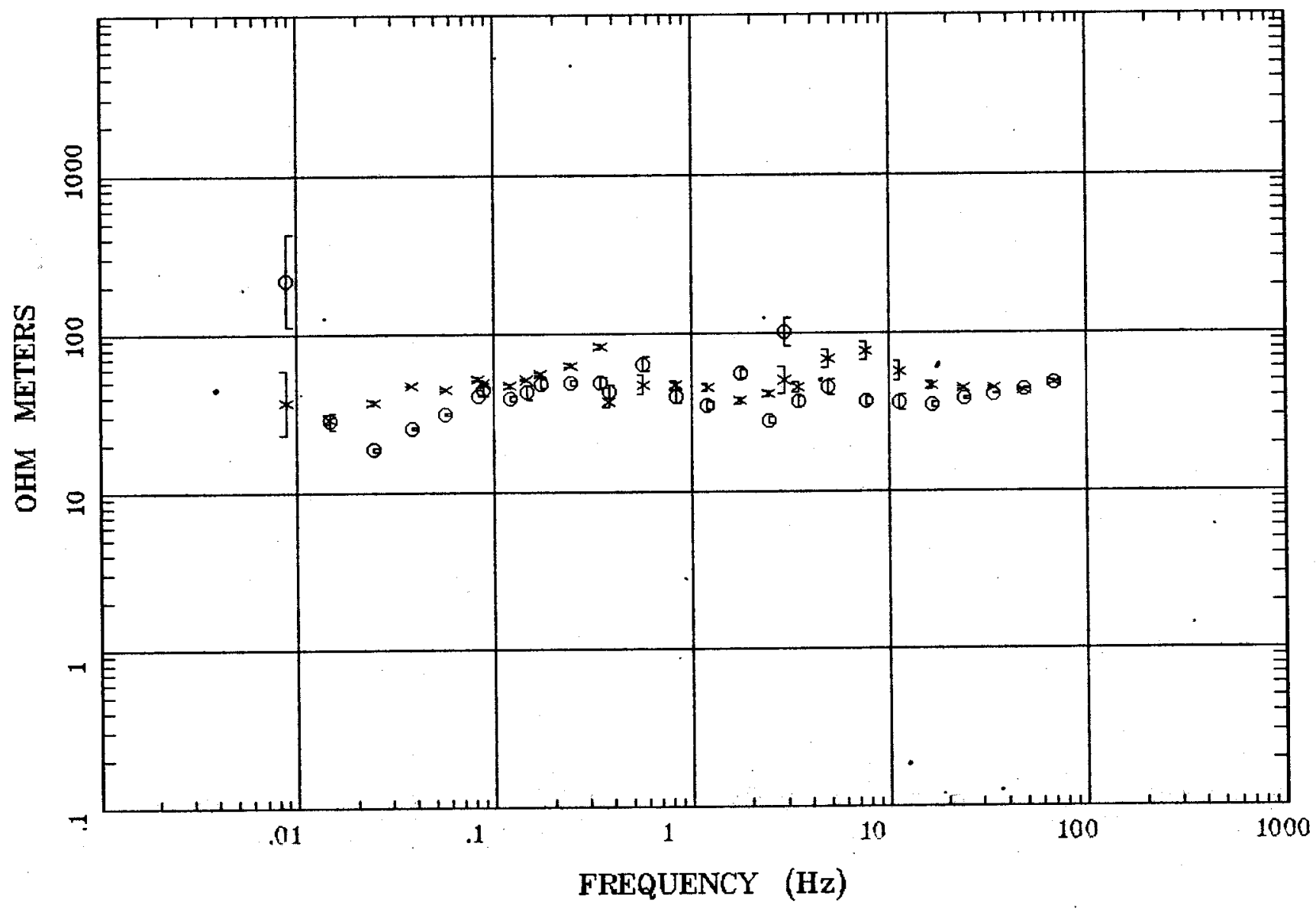

Client:

Remote: e-fld SE $35 \mathrm{~m}$

Acquired: 14:2 Sep 08, 2003 Survey Co:USGS
Rotation:

Filename: ap10m.avg

Channels: Ch1 Ch2 Ch3 Ch4 Ch5 Ch1 Ch2

Plotted: 14:28 Sep 24, 2003

< EMI - ElectroMagnetic Instruments > 


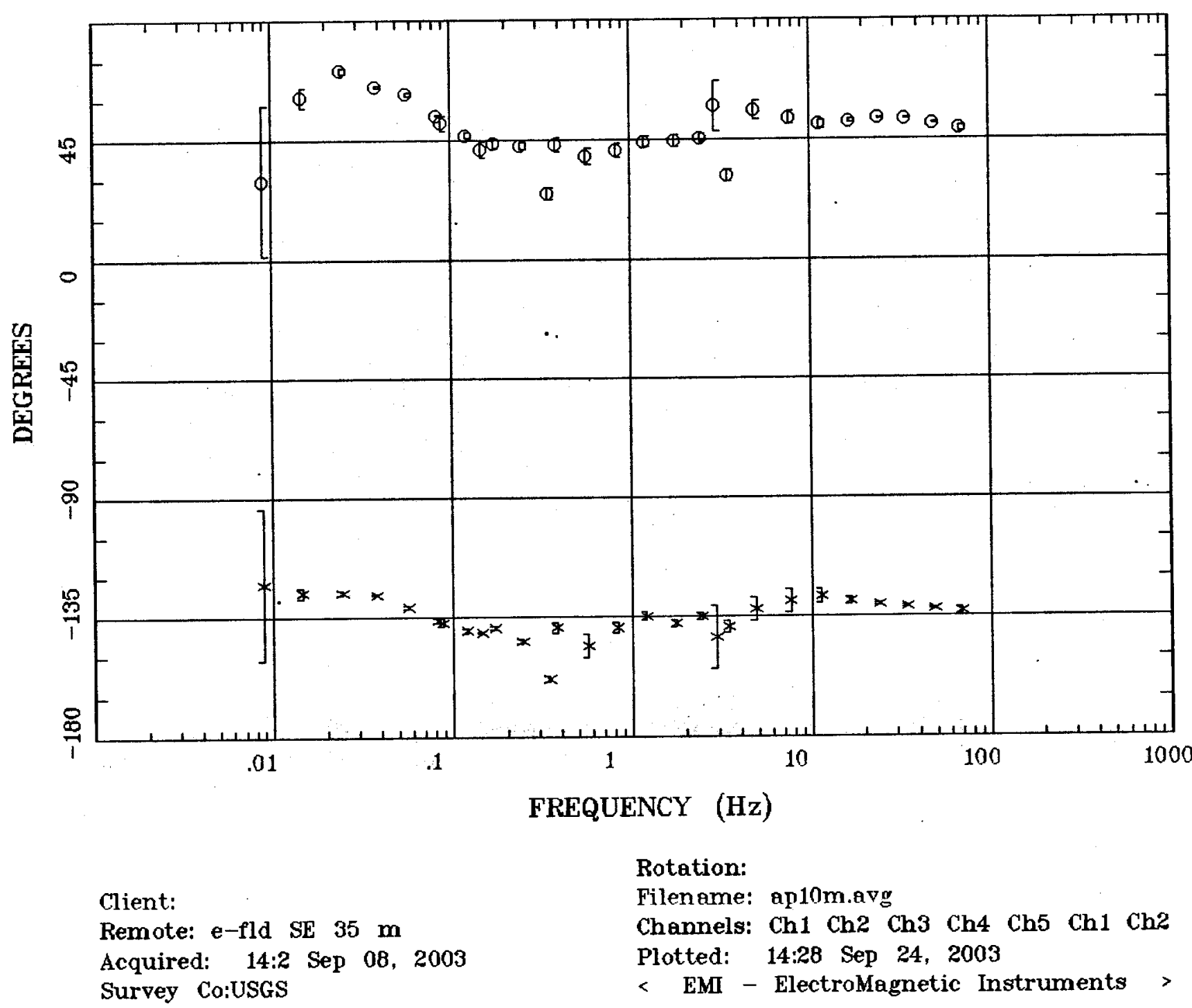




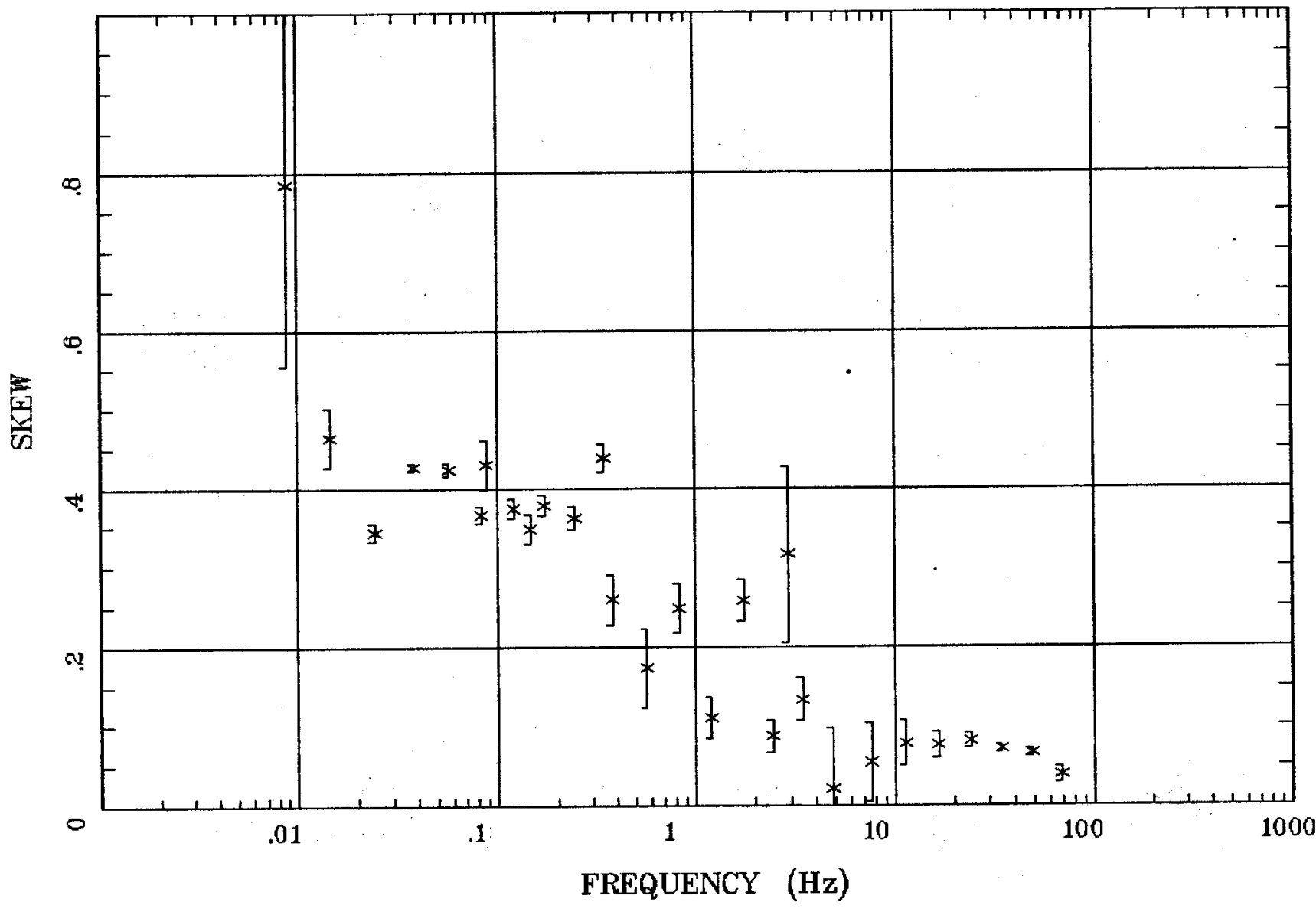

Client:

Remote: e-fld SE $35 \mathrm{~m}$

Acquired: 14:2 Sep 08, 2003 Survey Co:USGS
Rotation:

Filename: ap10m.avg

Channels: Ch1 Ch2 ch3 Ch4 Ch5 Ch1 Ch2 Plotted: 14:28 Sep 24, 2003

< EMI - ElectroMagnetic Instruments 
E MULT Coh.

Currie, NV $100 \mathrm{~K} \quad$ Station 10

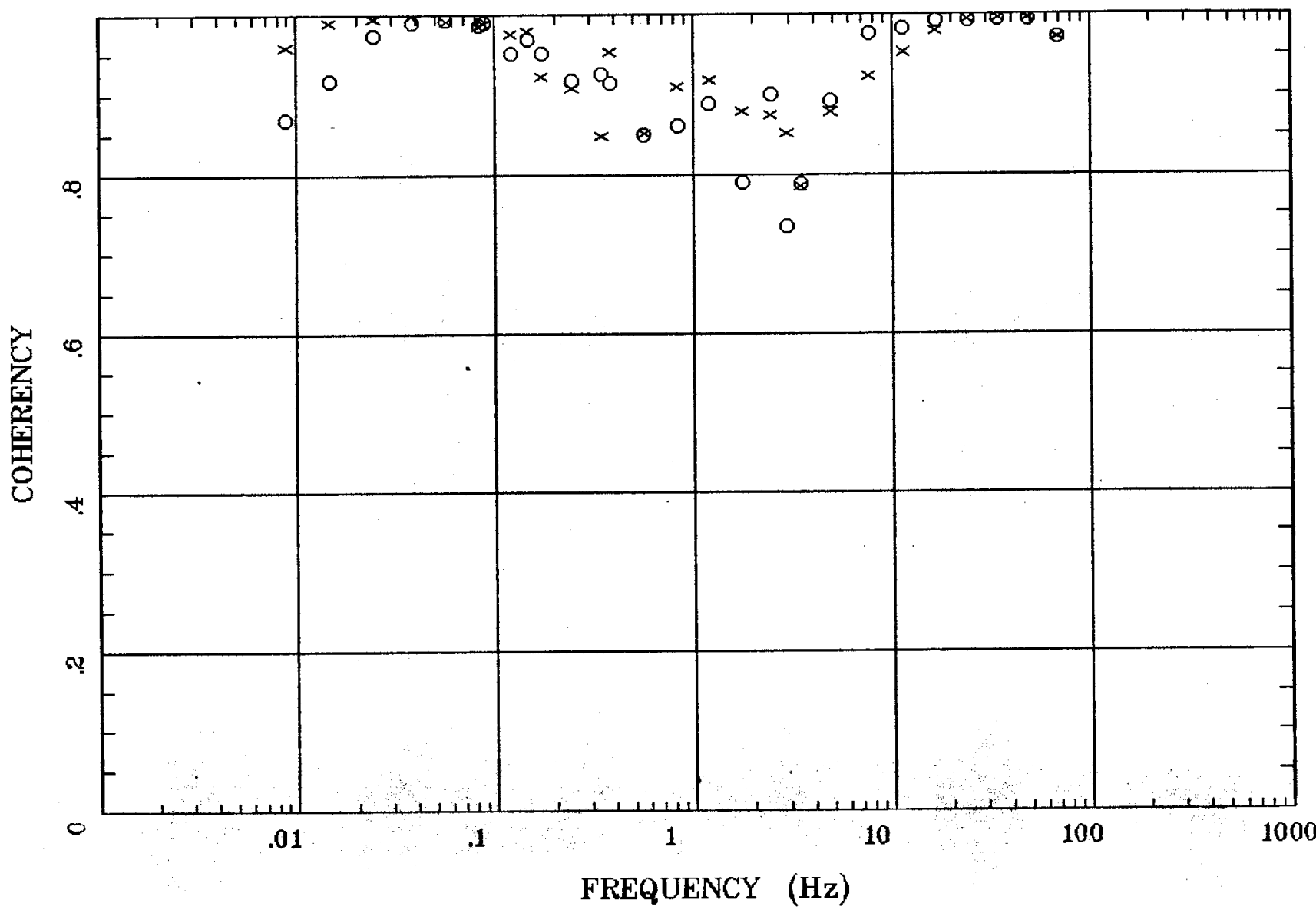

Client:

Remote: e-fld SE $35 \mathrm{~m}$

Acquired: 14:2 Sep 08, 2003

Survey Co:USGS
Rotation:

Filename: ap10m.avg

Channels: Ch1 Ch2 Ch3 Ch4 Ch5 Ch1 Ch2 Plotted: 14:28 Sep 24, 2003

< EMI - ElectroMagnetic Instruments 


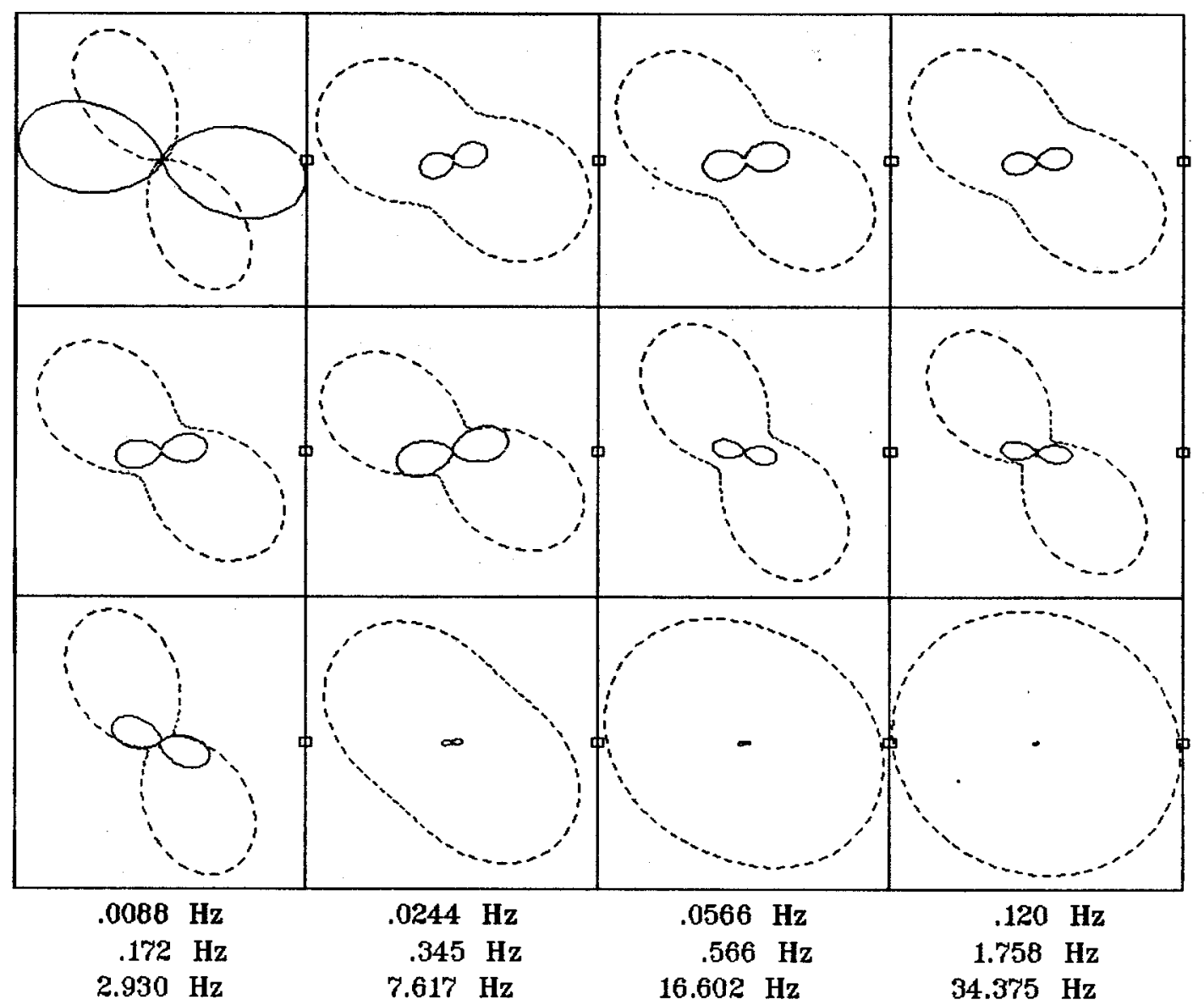

Client:

Remote: e-fld SE $35 \mathrm{~m}$

Acquired: 14:2 Sep 08, 2003 Survey Co:USGS
Rotation:

Filename: ap10m.avg

Channels: Ch1 Ch2 Ch3 Ch4 Ch5 Ch6 Ch7 Plotted: 08:38 Sep 24, 2003

< EMI - ElectroMagnetic Instruments > 


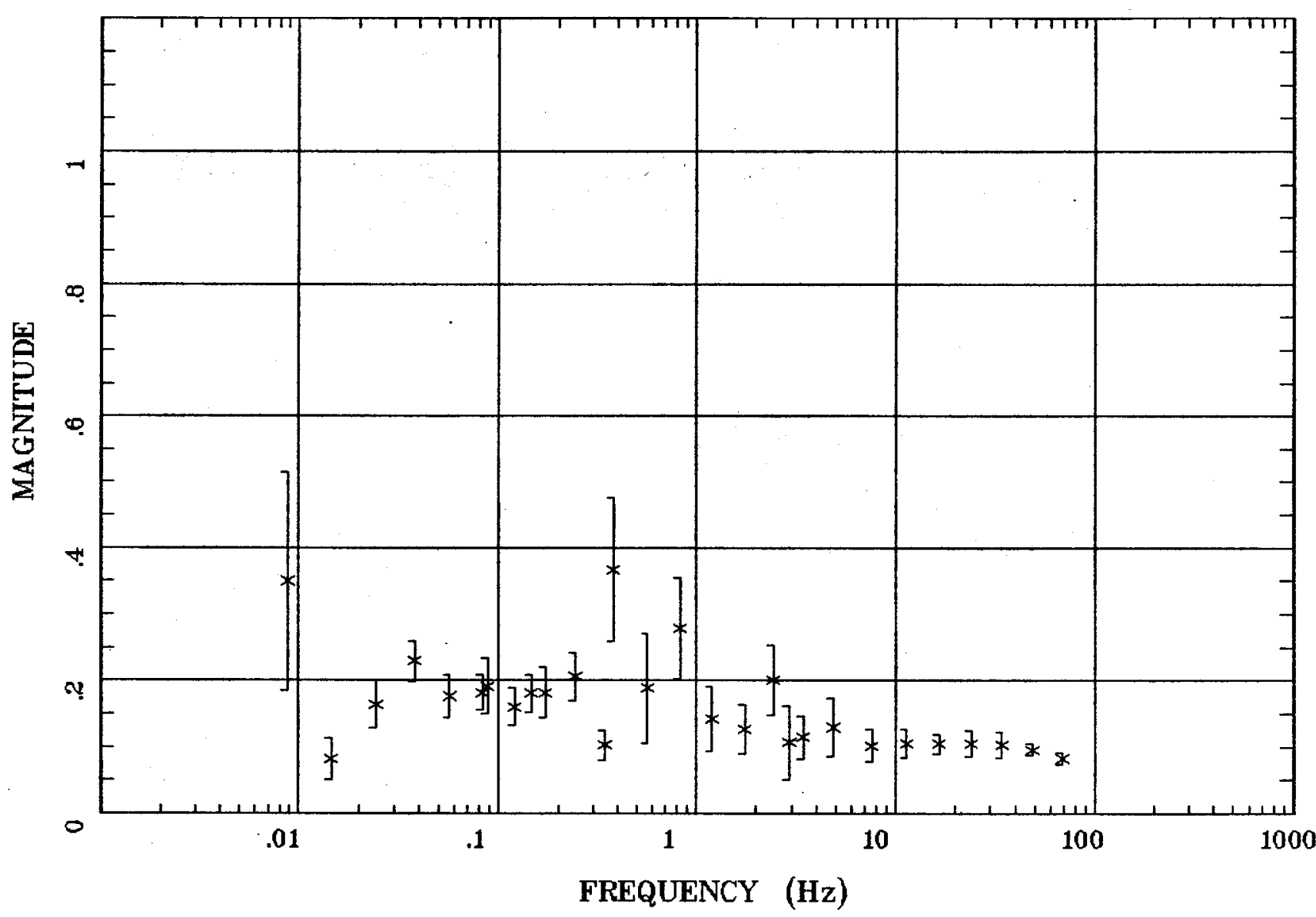

Client:

Remote: e-fld SE $35 \mathrm{~m}$

Acquired: 14:2 Sep 08, 2003

Survey Co:USGS
Rotation:

Filename: ap10m.avg

Channels: Ch1 Ch2 Ch3 Ch4 Ch5 Ch6 Ch7

Plotted: 08:39 Sep 24, 2003

$<$ EMI - ElectroMagnetic Instruments > 


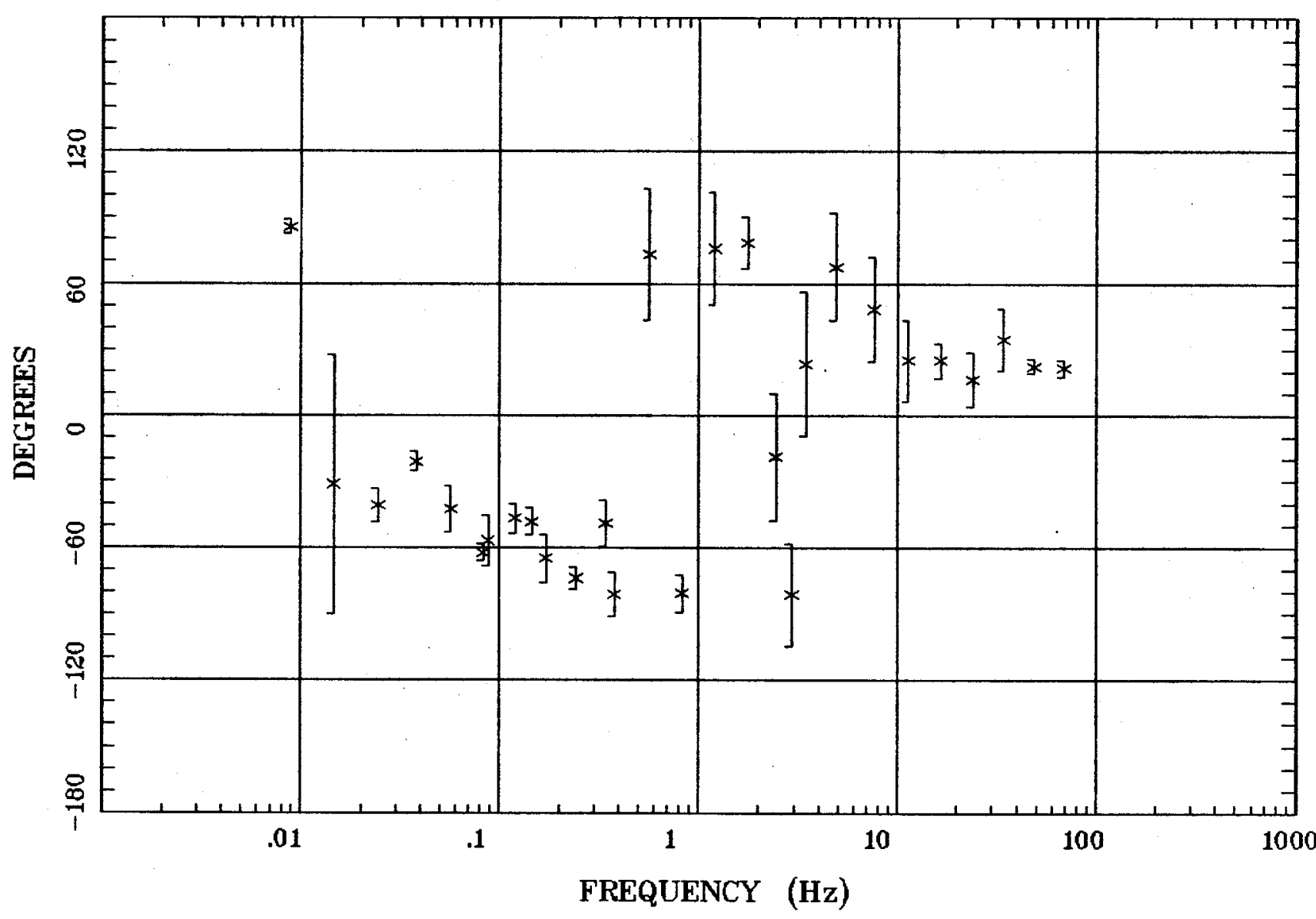

Client:

Remote: e-fld SE $35 \mathrm{~m}$

Acquired: 14:2 Sep 08, 2003

Survey Co:USGS
Rotation:

Filename: ap10m.avg

Channels: Ch1 Ch2 Ch3 Ch4 Ch5 Ch6 Ch7

Plotted: 08:39 Sep 24, 2003

$<$ EMI - ElectroMagnetic Instruments 


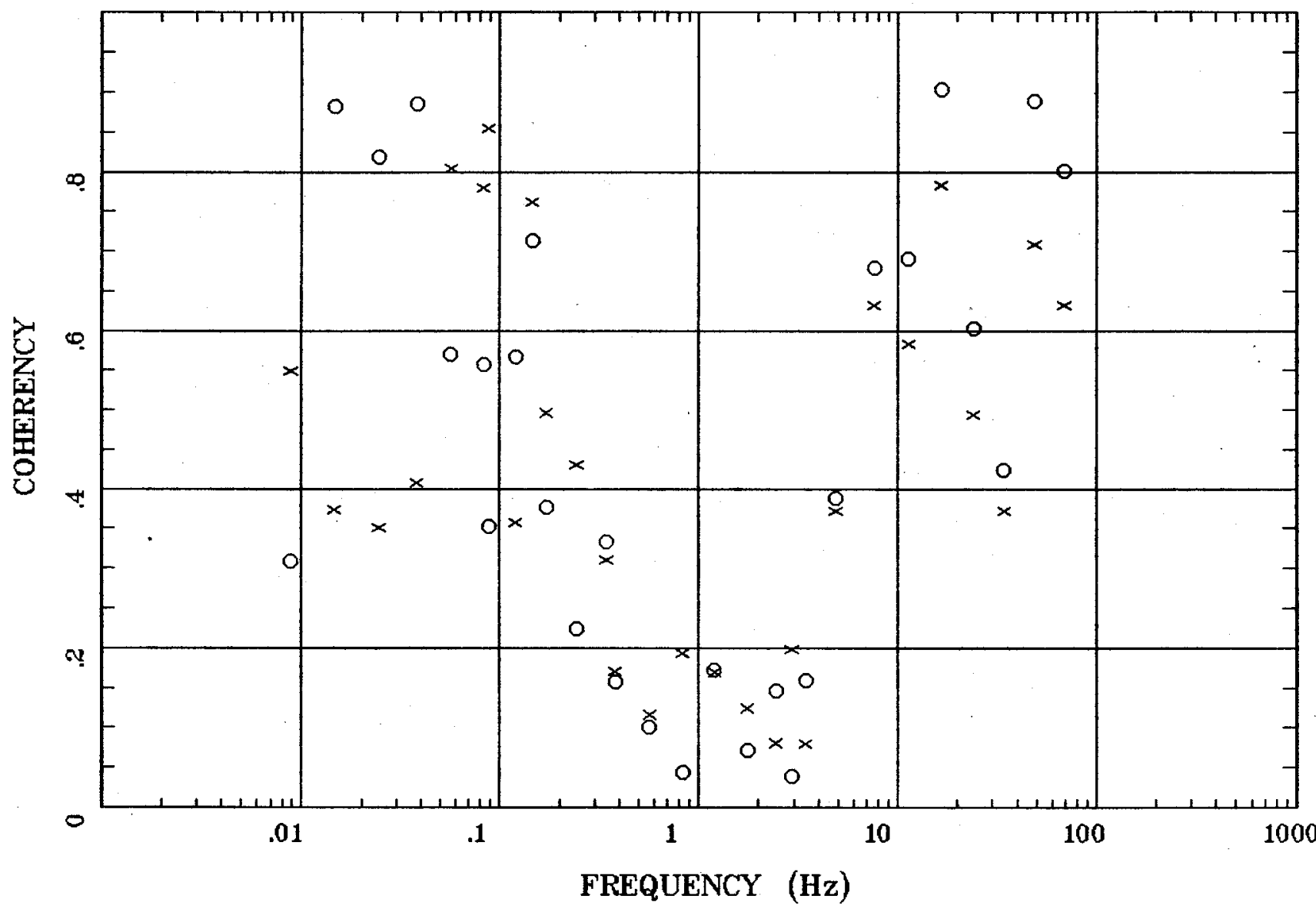

Client:

Remote: e-fld SE $35 \mathrm{~m}$

Acquired: 14:2 $\operatorname{Sep} 08,2003$ Survey Co:USGS
Rotation:

Filename: ap $10 \mathrm{~m}$.avg

Channels: Ch1 Ch2 Ch3 Ch4 Ch5 Ch6 Chr Plotted: $\quad 08: 39$ Sep 24, 2003

$<$ EMI - ElectroMagnetic Instruments 


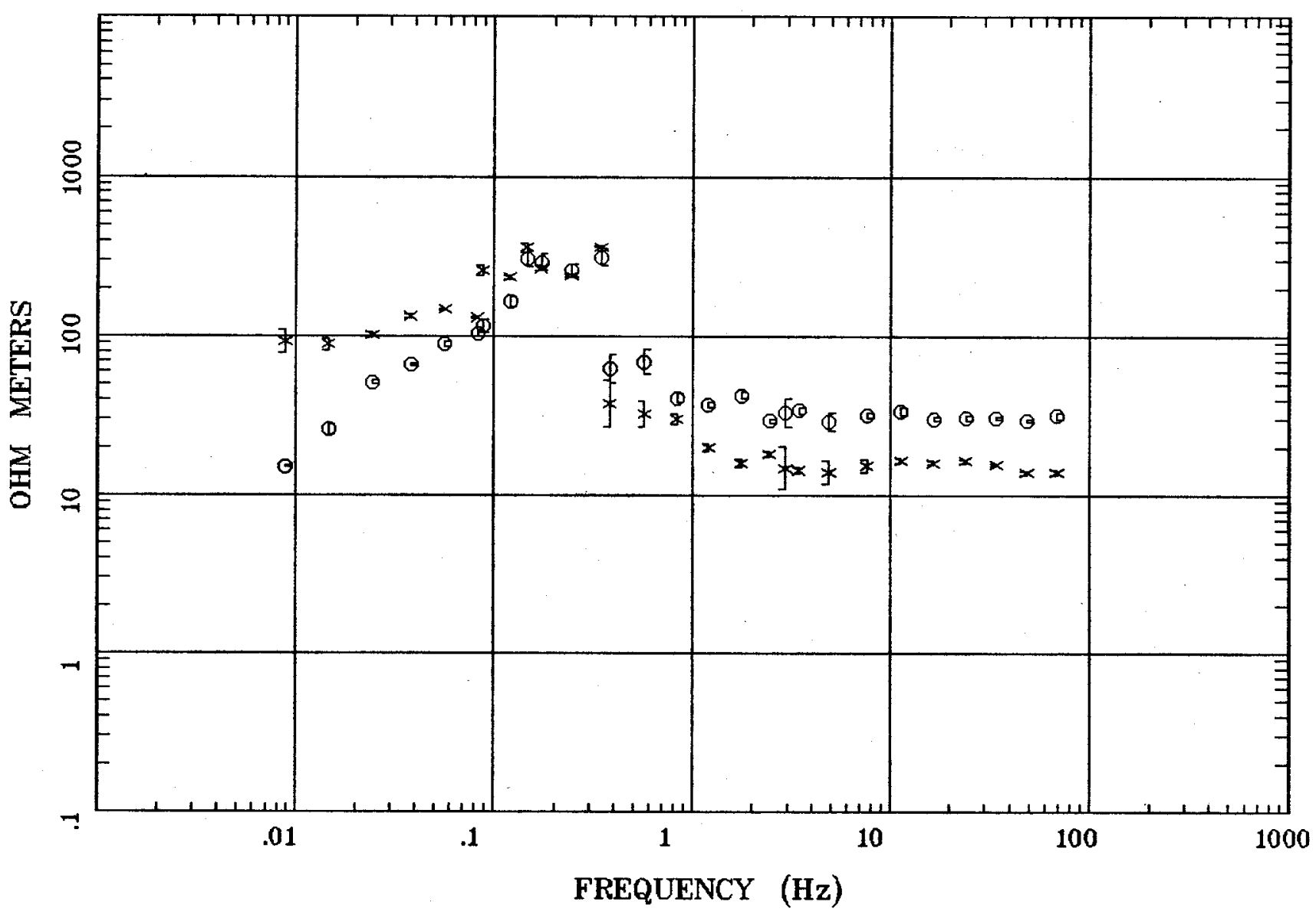

Client:

Remate: e-fld south $90 \mathrm{~m}$ Acquired: 11:0 Sep 12, 2003 Survey Co:USGS
Rotation:

Filename: ap11n.avg Channels: Ch1 Ch2 Ch3 Ch4 Ch5 Ch6 Ch7 Plotted: 08:46 Sep 24, 2003

$<$ EMI - ElectroMagnetic Instruments 


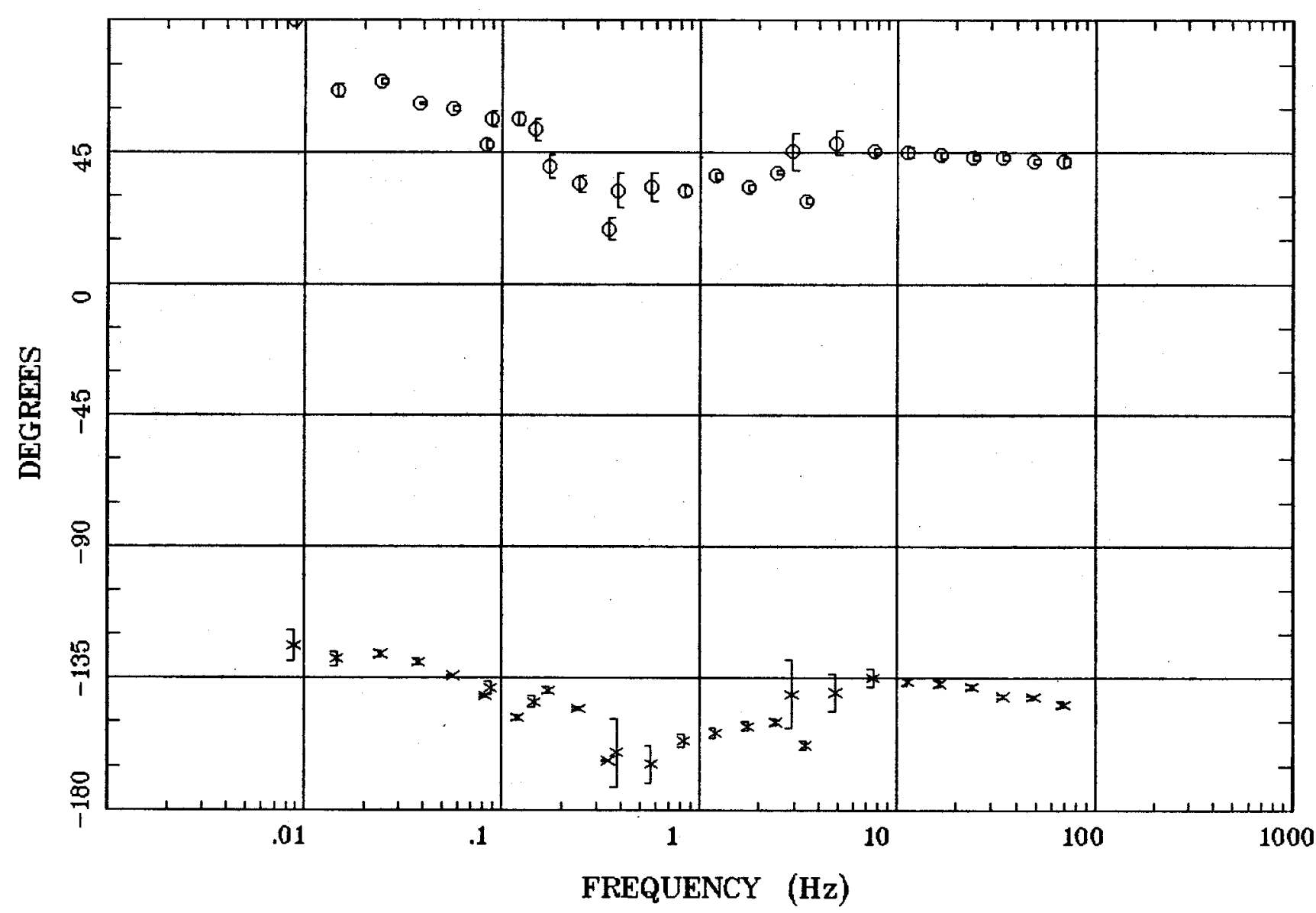

Client:

Remote: e-fld south $90 \mathrm{~m}$ Acquired: 11:0 Sep 12, 2003 Survey Co:USGS
Rotation:

Filename: ap11n.avg

Channels: Ch1 Ch2 Ch3 Ch4 Ch5 Ch6 Ch7 Plotted: 08:47 Sep 24, 2003

$<$ EMI - ElectroMagnetic Instruments 


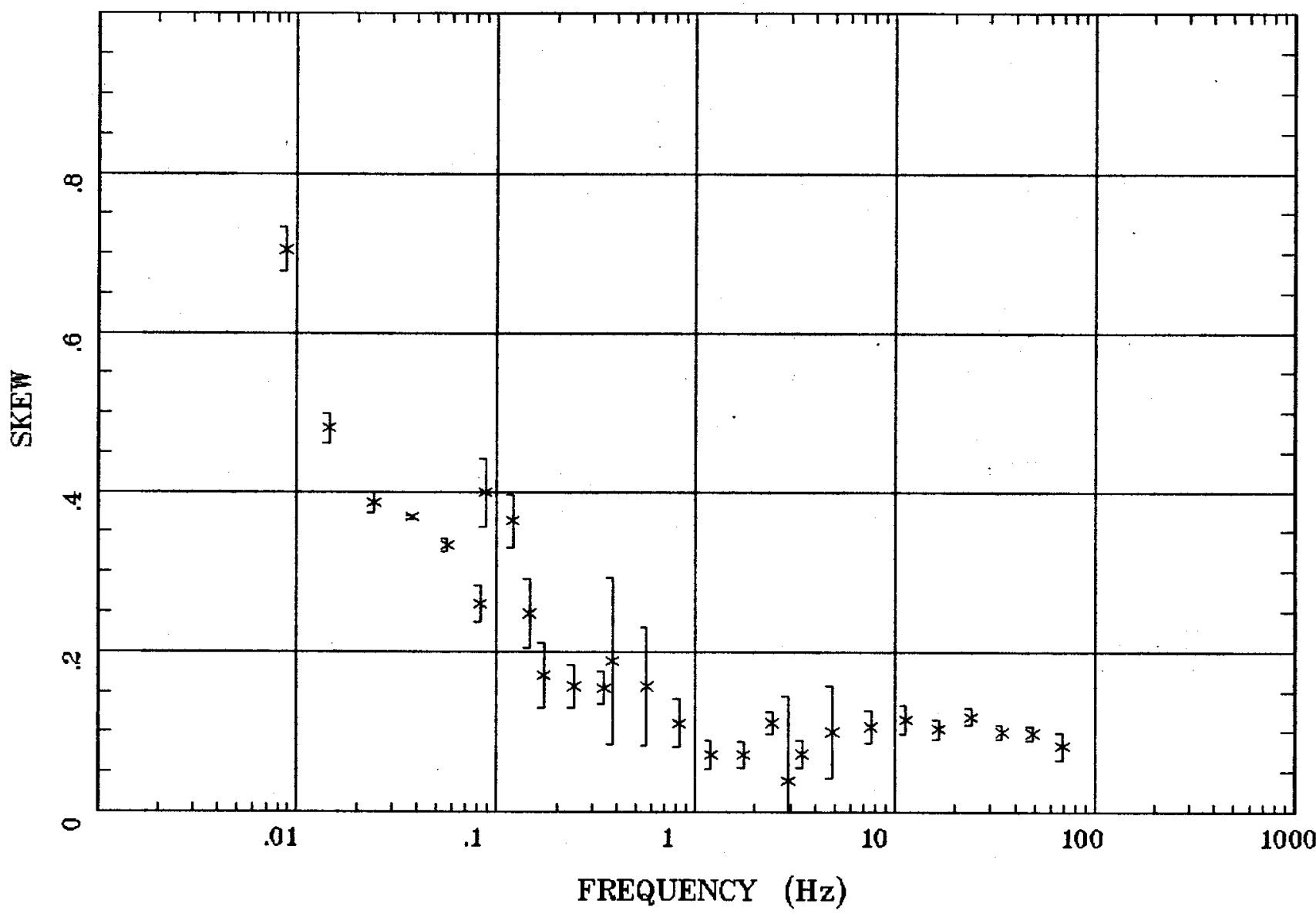

Client:

Remote: e-fld south $90 \mathrm{~m}$ Acquired: 11:0 Sep 12, 2003 Survey Co:USGS
Rotation:

Filename: ap11n.avg

Channels: Ch1 Ch2 Ch3 Ch4 Ch5 Ch6 Ch7 Plotted: 08:47 Sep 24, 2003

< EMI - ElectroMagnetic Instruments 
E MULT Coh.

Currie, NV $100 \mathrm{~K}$

Station 11

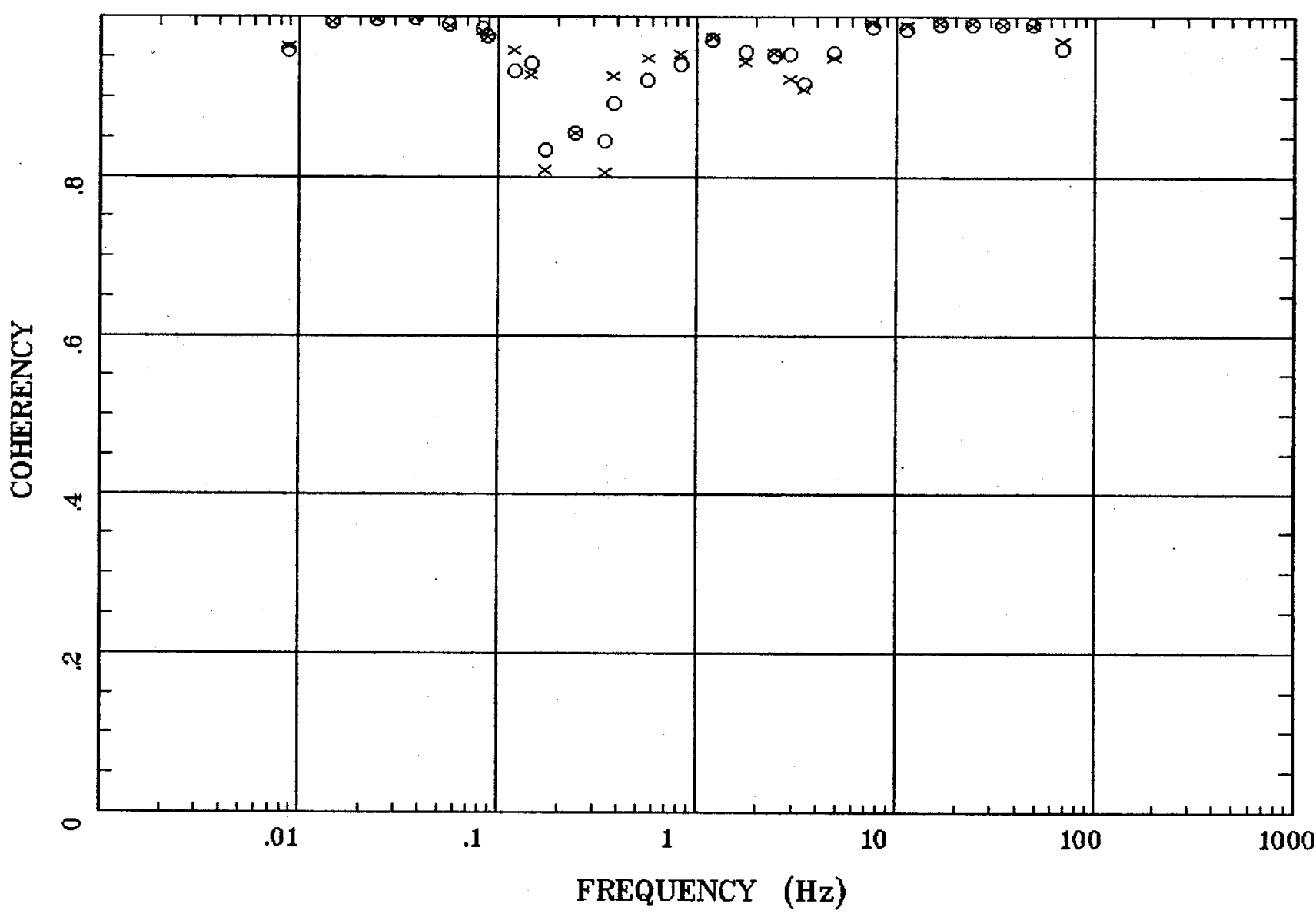

Client:

Remote: e-fld south $90 \mathrm{~m}$

Acquired: 11:0 Sep 12, 2003

Survey Co:USGS
Rotation:

Filename: ap11n.avg

Channels: Ch1 Ch2 Ch3 Ch4 Ch5 Ch6 Ch7

Plotted: 08:47 Sep 24, 2003

$<$ EMI - ElectroMagnetic Instruments 


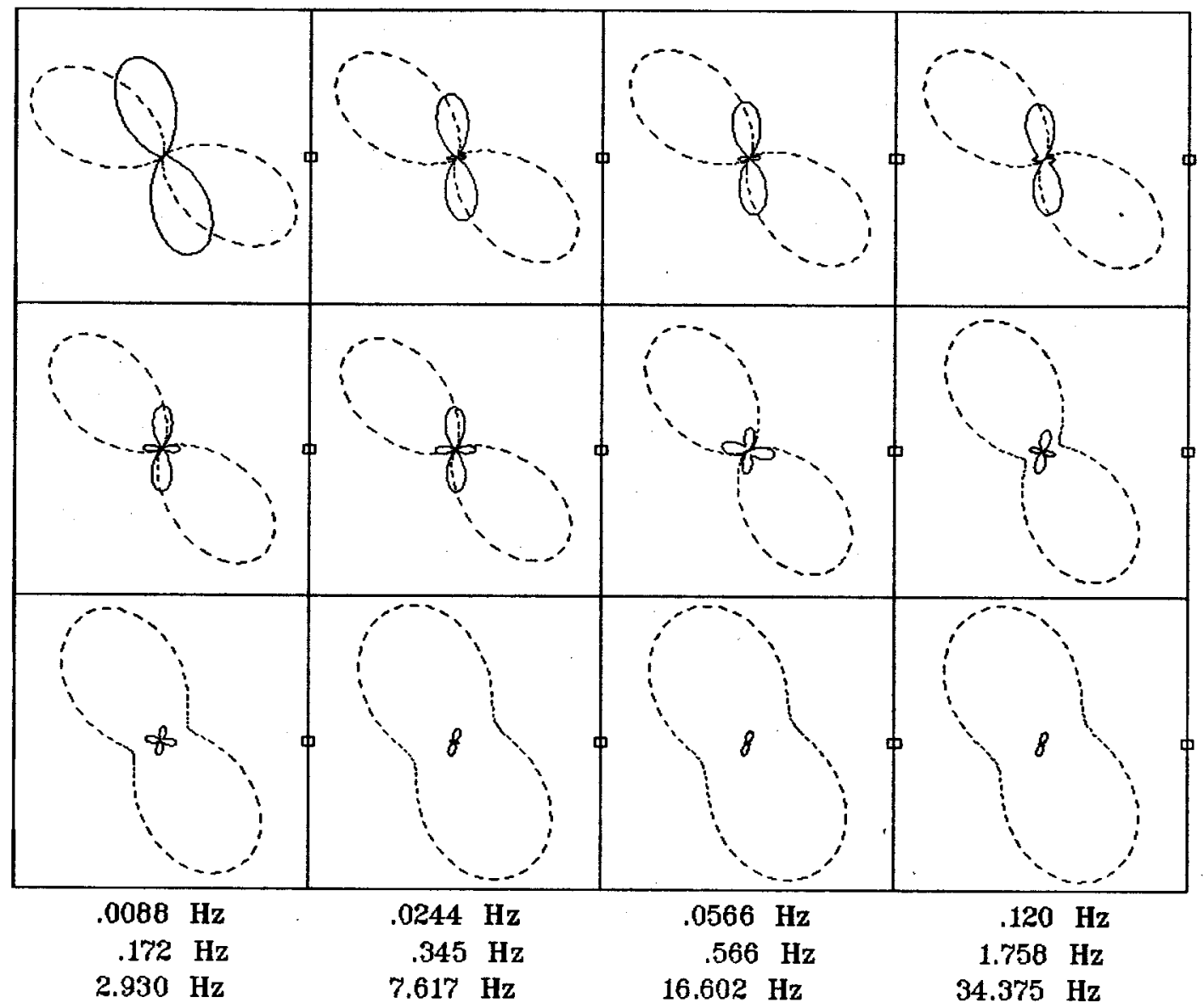

Client:

Remote: e-fld south $90 \mathrm{~m}$ Acquired: 11:0 Sep 12, 2003 Survey Co:USGS
Rotation:

Filename: ap11n.avg

Channels: Ch1 Ch2 Ch3 Ch4 Ch5 Ch6 Ch7 Plotted: 08:47 Sep 24, 2003

< EMI - ElectroMagnetic Instruments 


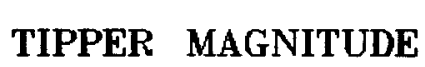

Currie, NV $100 \mathrm{~K} \quad$ Station 11

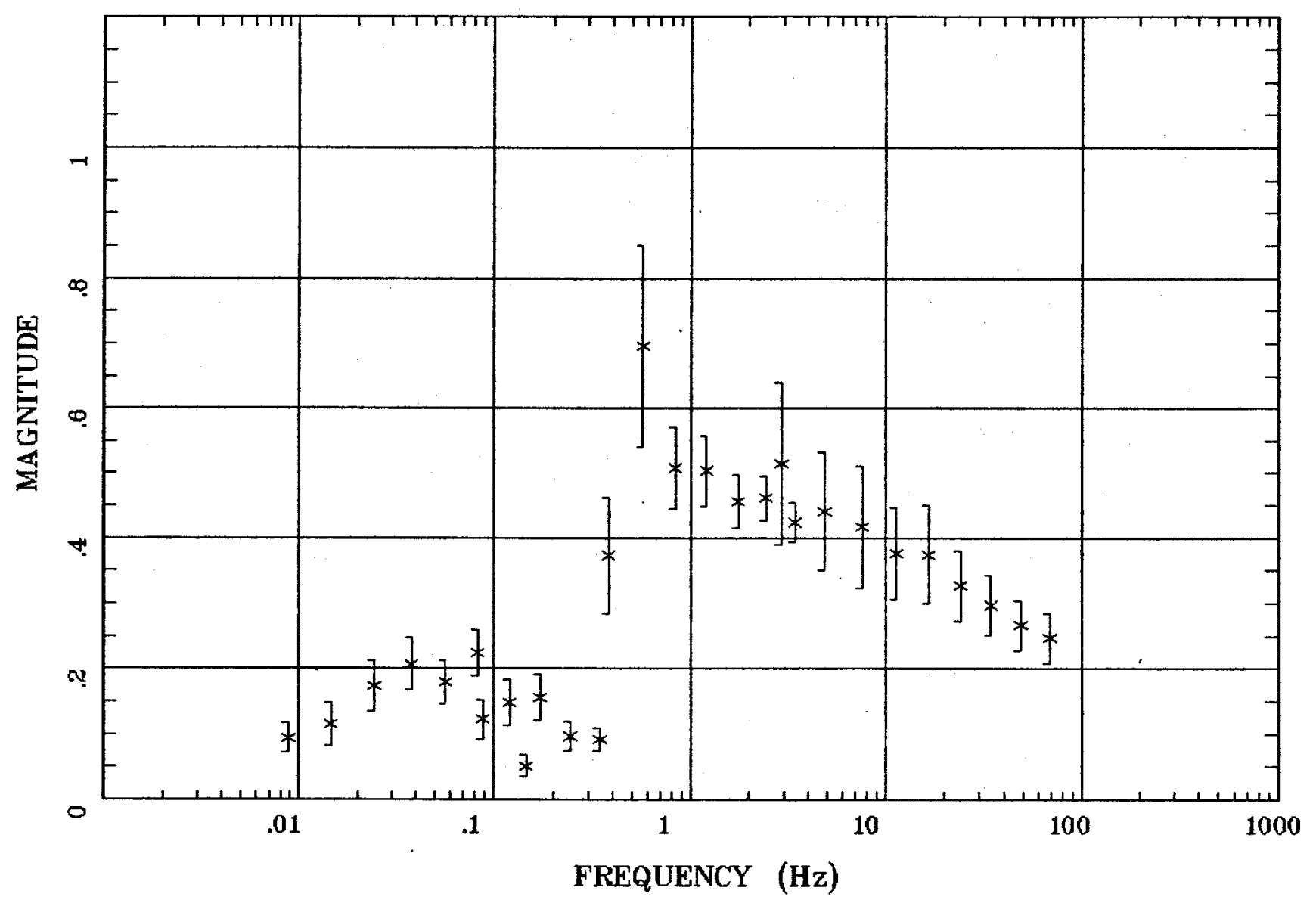

Client:

Remote: e-fld south $90 \mathrm{~m}$

Acquired: 11:0 Sep 12, 2003

Rotation:

Filename: ap11n.avg

Channels: Ch1 Ch2 Ch3 Ch4 Ch5 Ch6 Ch7

Survey Co:USGS

Plotted: 08:47 Sep 24, 2003

< EMI - ElectroMagnetic Instruments 


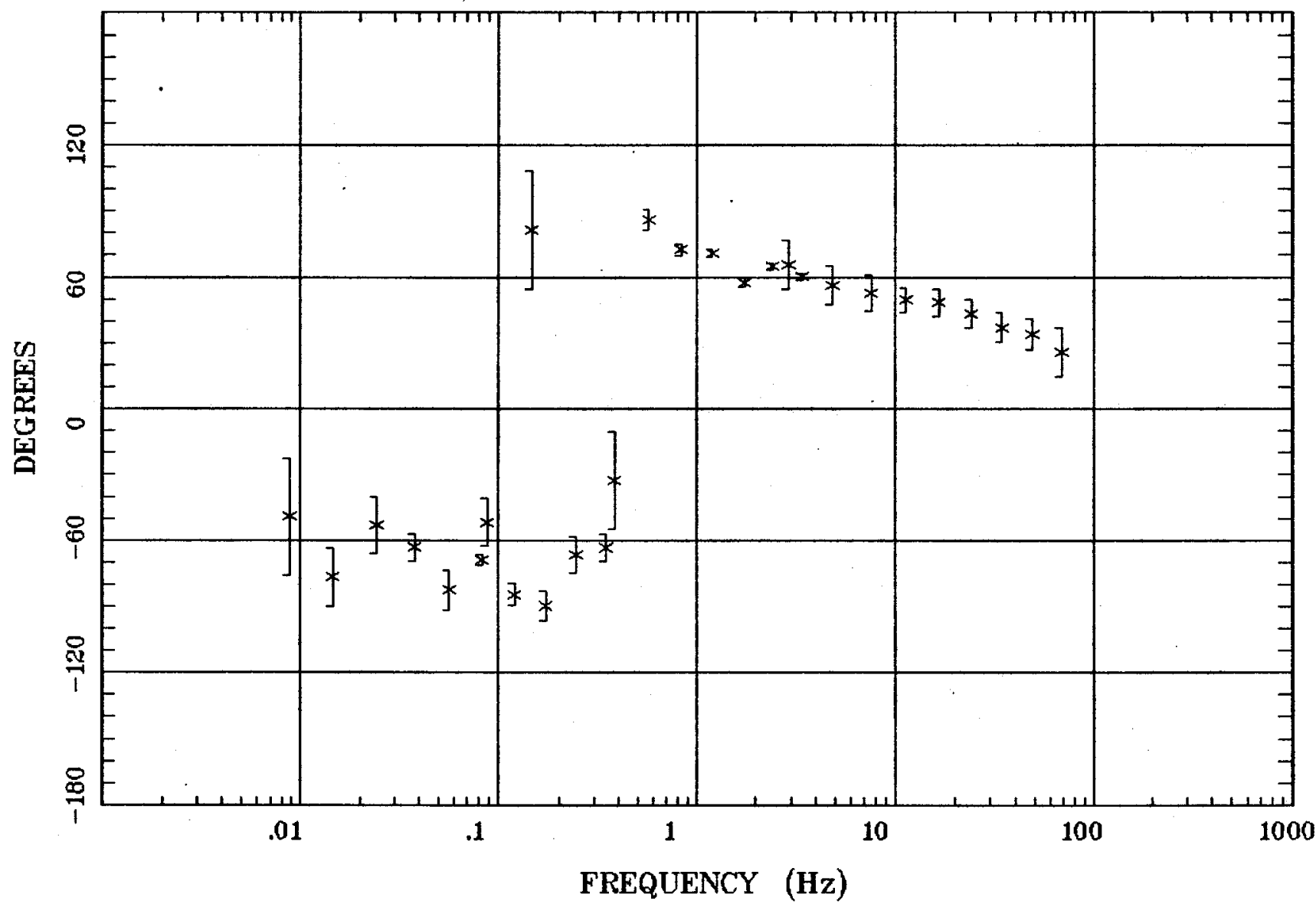

Client:

Remote: e-fld south $90 \mathrm{~m}$ Acquired: 11:0 Sep 12, 2003 Survey Co:USGS
Rotation:

Filename: ap11n.avg

Channels: Ch1 Ch2 Ch3 Ch4 Ch5 Ch6 Ch7 Plotted: 08:47 Sep 24, 2003

$<$ EMI - ElectroMagnetic Instruments 


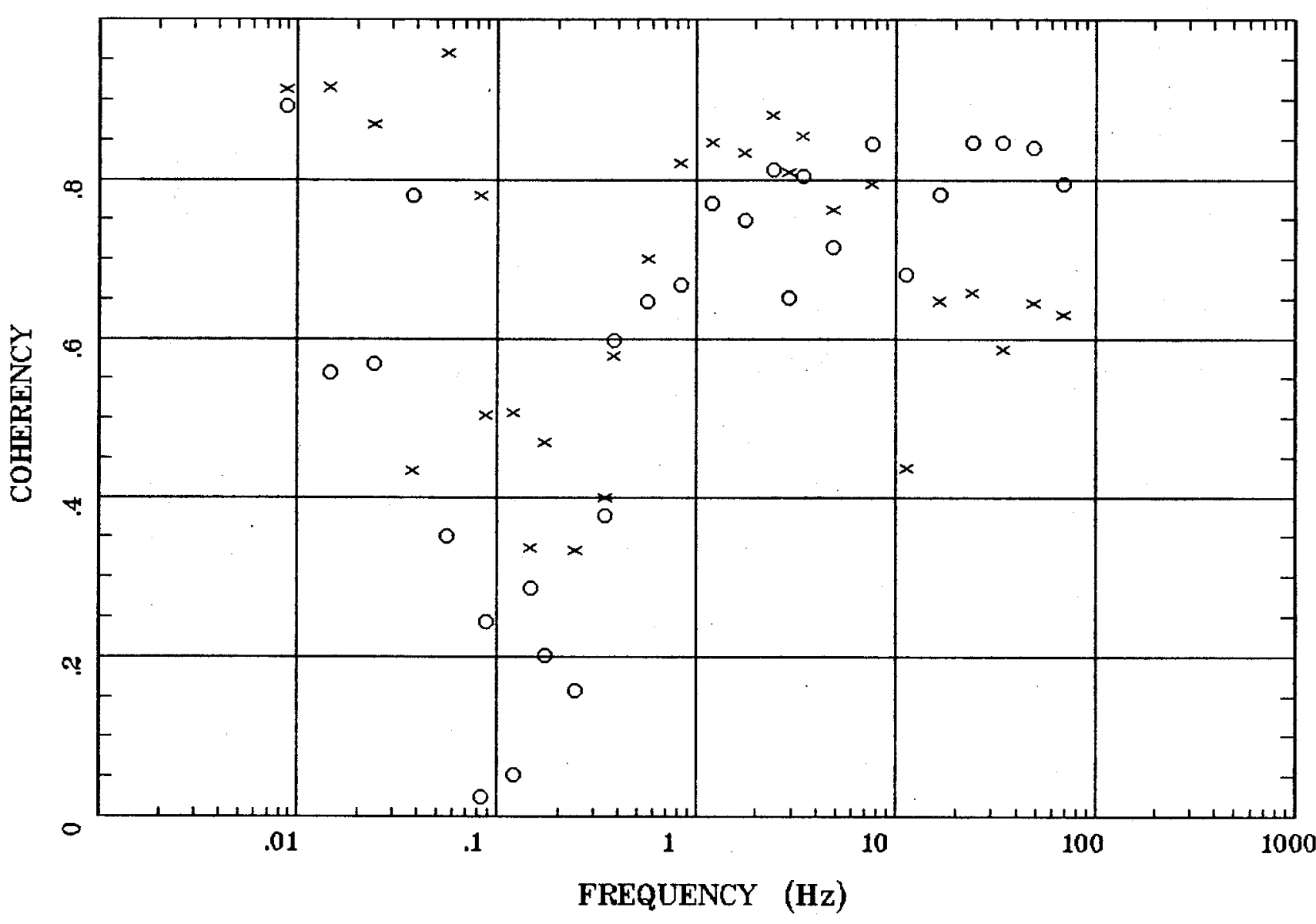

Client:

Remote: e-fld south $90 \mathrm{~m}$ Acquired: 11:0 Sep 12, 2003 Survey Co:USGS
Rotation:

Filename: ap11n.avg

Channels: Ch1 Ch2 Ch3 ch4 Ch5 Ch6 Ch7 Plotted: 08:47 Sep 24, 2003

< EMI - ElectroMagnetic Instruments 


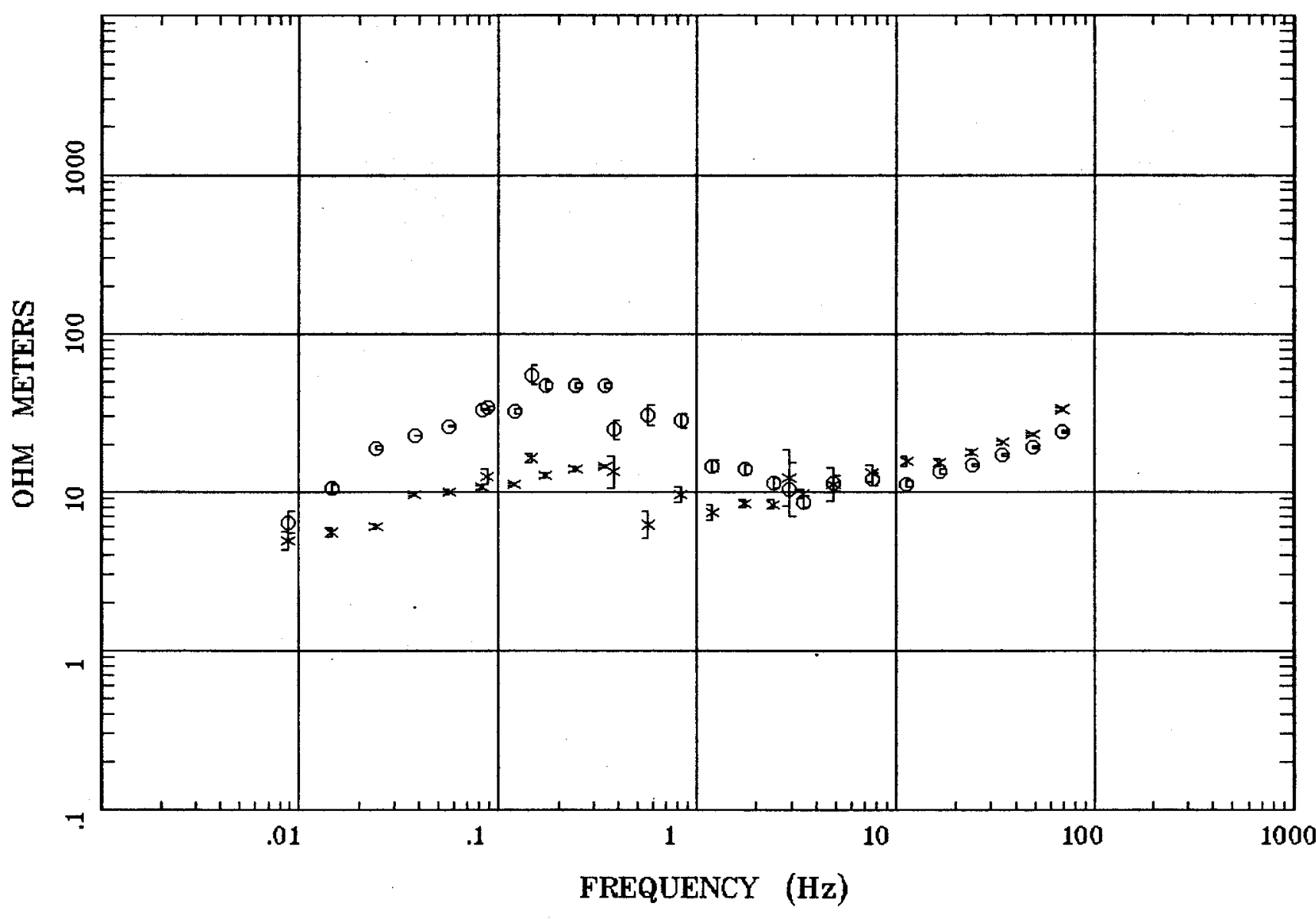

Client:

Remote: e-fld east $90 \mathrm{~m}$ Acquired: 10:3 Sep 09, 2003 Survey Ca:USGS
Rotation:

Filename: ap12m.avg

Channels: Ch1 Ch2 Ch3 Ch4 Ch5 Ch6 Ch7 Plotted: 08:47 Sep 24, 2003

$<$ EMI - ElectroMagnetic Instruments 


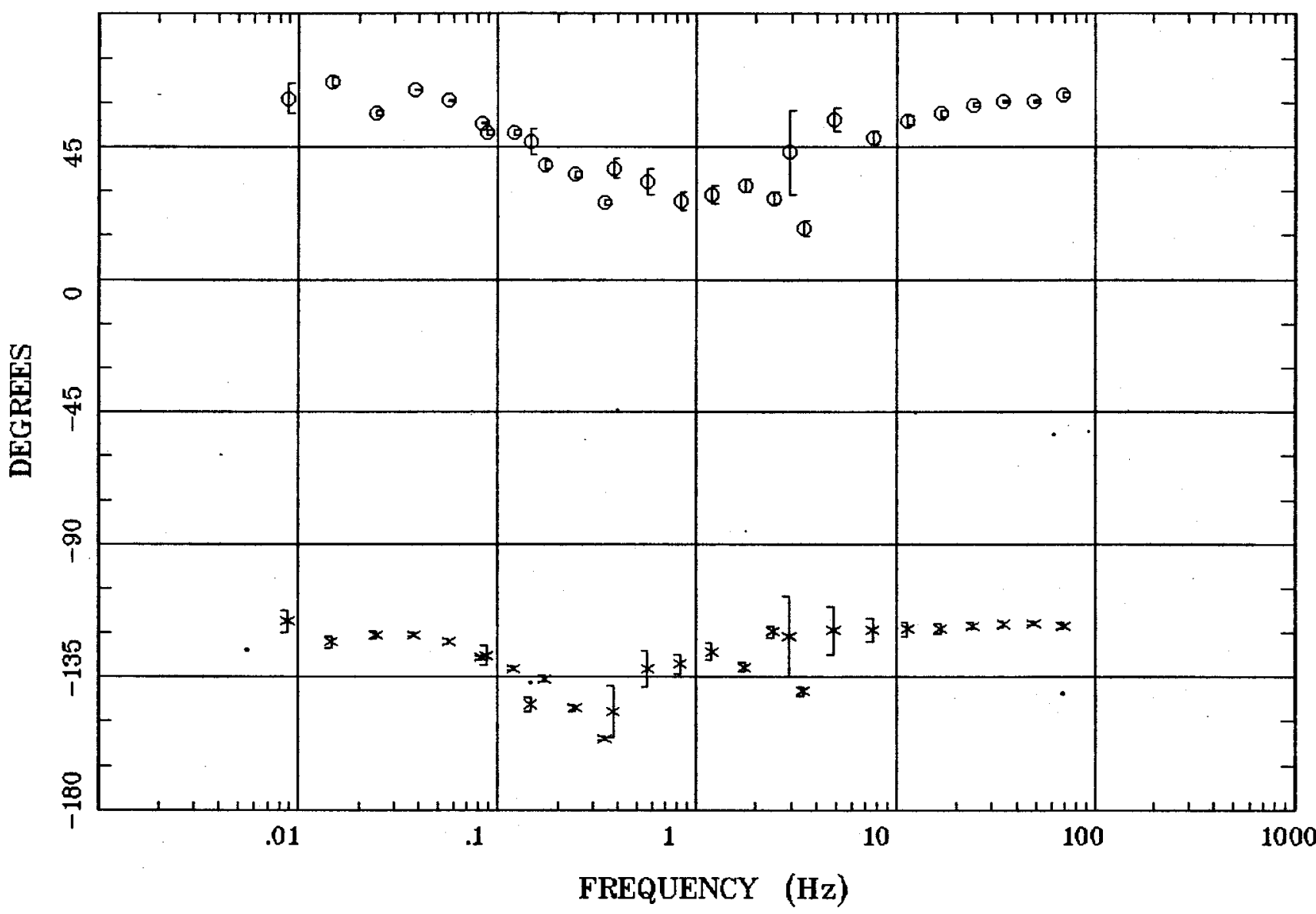

Client:

Remote: e-fld east $90 \mathrm{~m}$ Acquired: 10:3 Sep 09, 2003 Survey co:USGS
Rotation:

Filename: ap12m.avg

Channels: Ch1 Ch2 Ch3 Ch4 Ch5 Ch6 Ch7 Plotted: 08:47 Sep 24, 2003

< EMI - ElectroMagnetic Instruments 


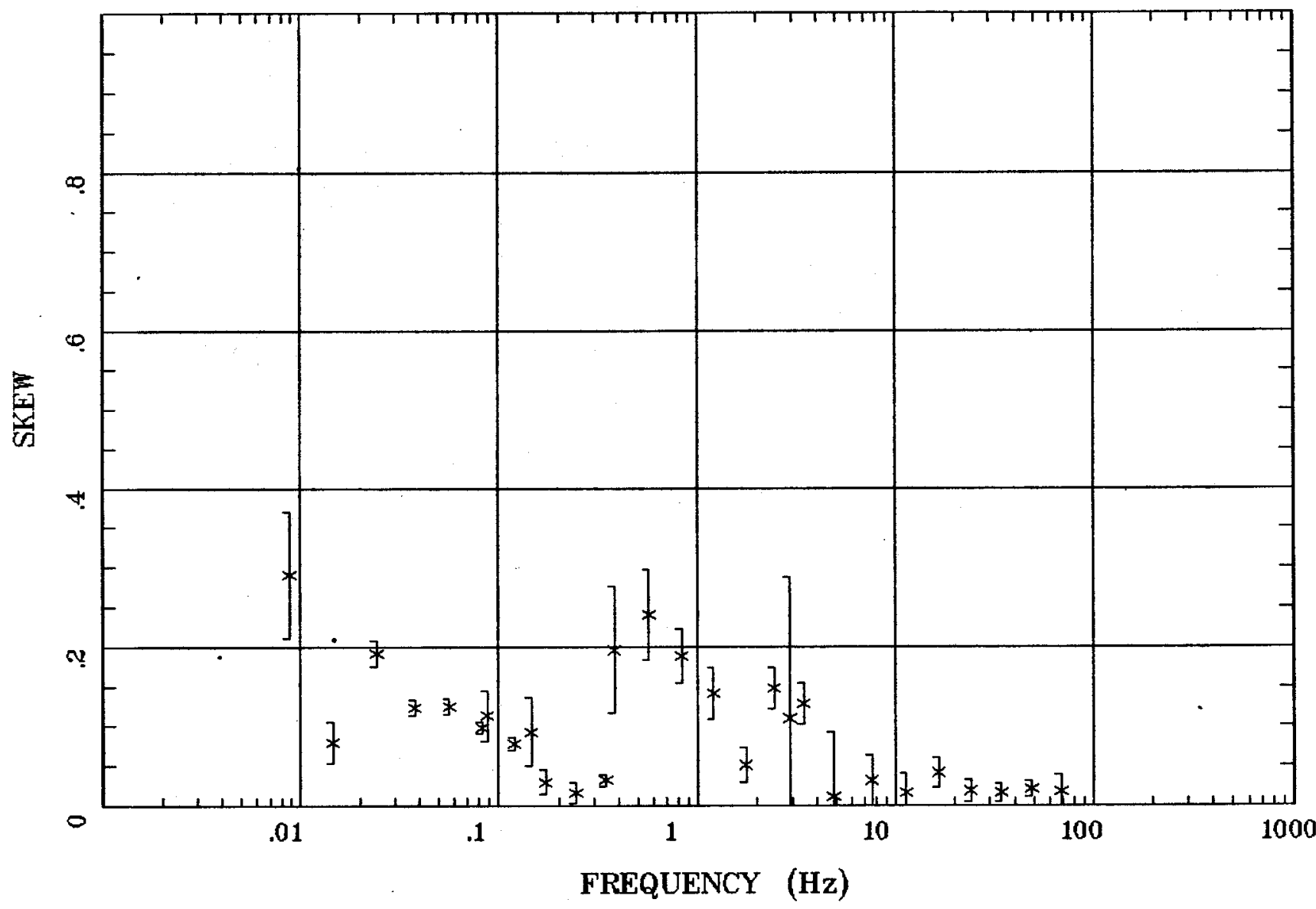

Client:

Remote: e-fld east $90 \mathrm{~m}$ Acquired: 10:3 Sep 09, 2003 Survey Co:USGS
Rotation:

Filename: ap12m.avg

Channels: Ch1 Ch2 Ch3 Ch4 Ch5 Ch6 Ch7 Platted: 08:47 Sep 24, 2003

$<$ EMI - ElectroMagnetic Instruments 


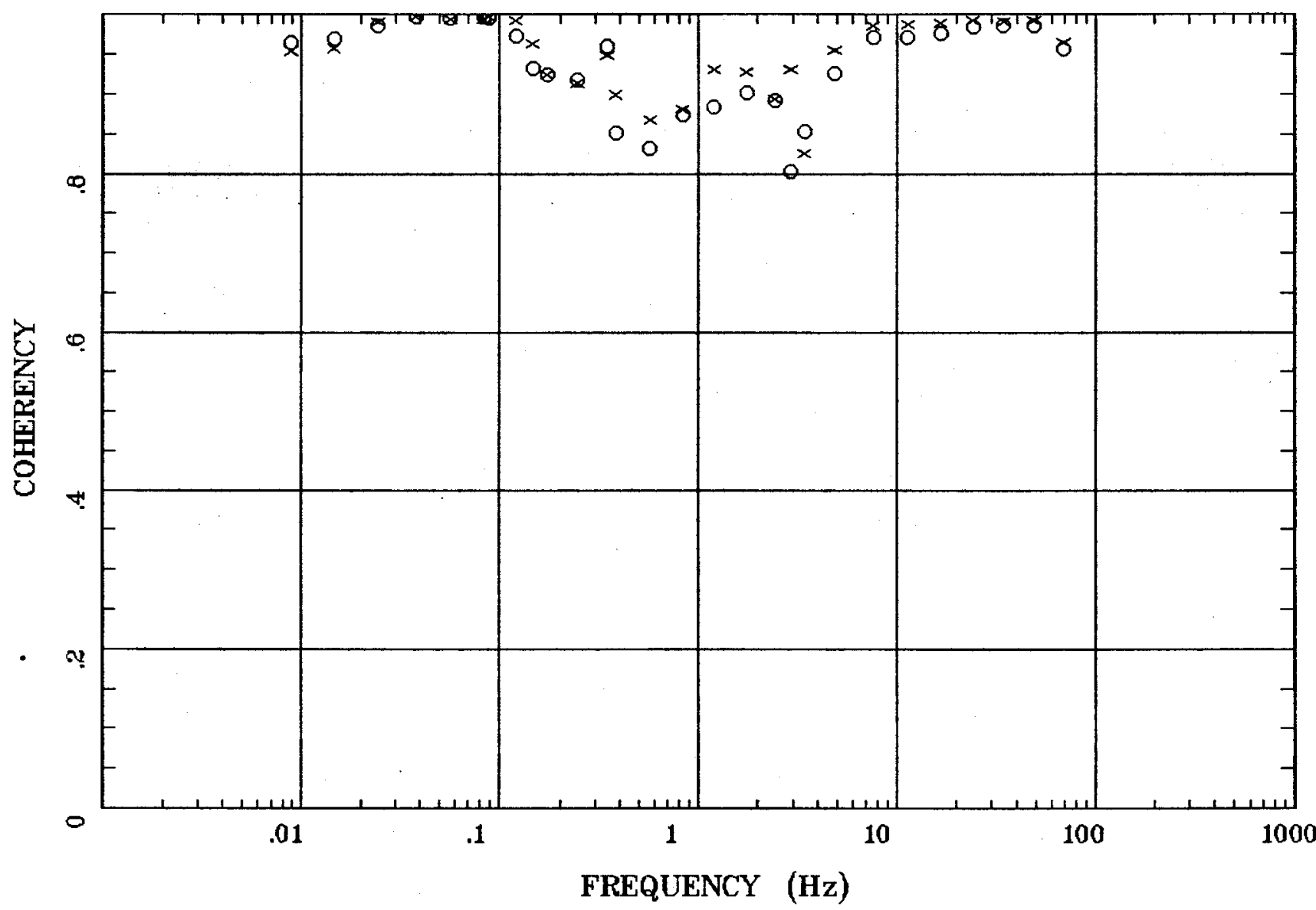

Client:

Remote: e-fld east $90 \mathrm{~m}$ Acquired: 10:3 Sep 09, 2003 Survey Co:USGS
Rotation:

Filename: ap12m.avg

Channels: Ch1 Ch2 Ch3 ch4 Ch5 Ch6 Ch7

Plotted: 08:48 Sep 24, 2003

$<$ EMI - ElectroMagnetic Instruments 


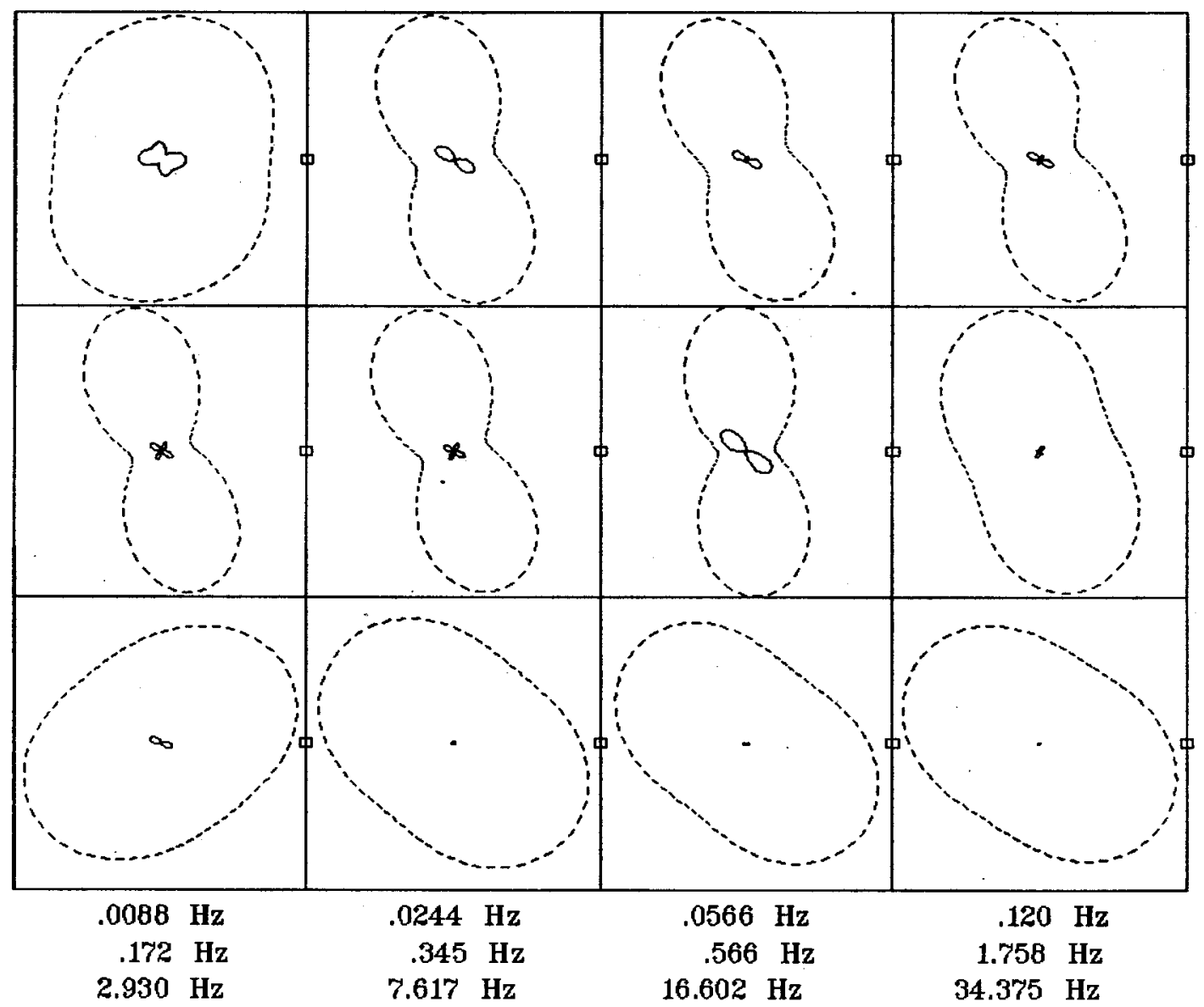

Rotation:

Client:

Remote: e-fld east $90 \mathrm{~m}$ Acquired: 10:3 Sep 09, 2003 Survey Ca:USGS
Filename: ap12m.avg

Channels: Ch1 Ch2 Ch3 Ch4 Ch5 Ch6 Ch7 Plotted: 08:48 Sep 24, 2003

< EMI - ElectroMagnetic Instruments > 


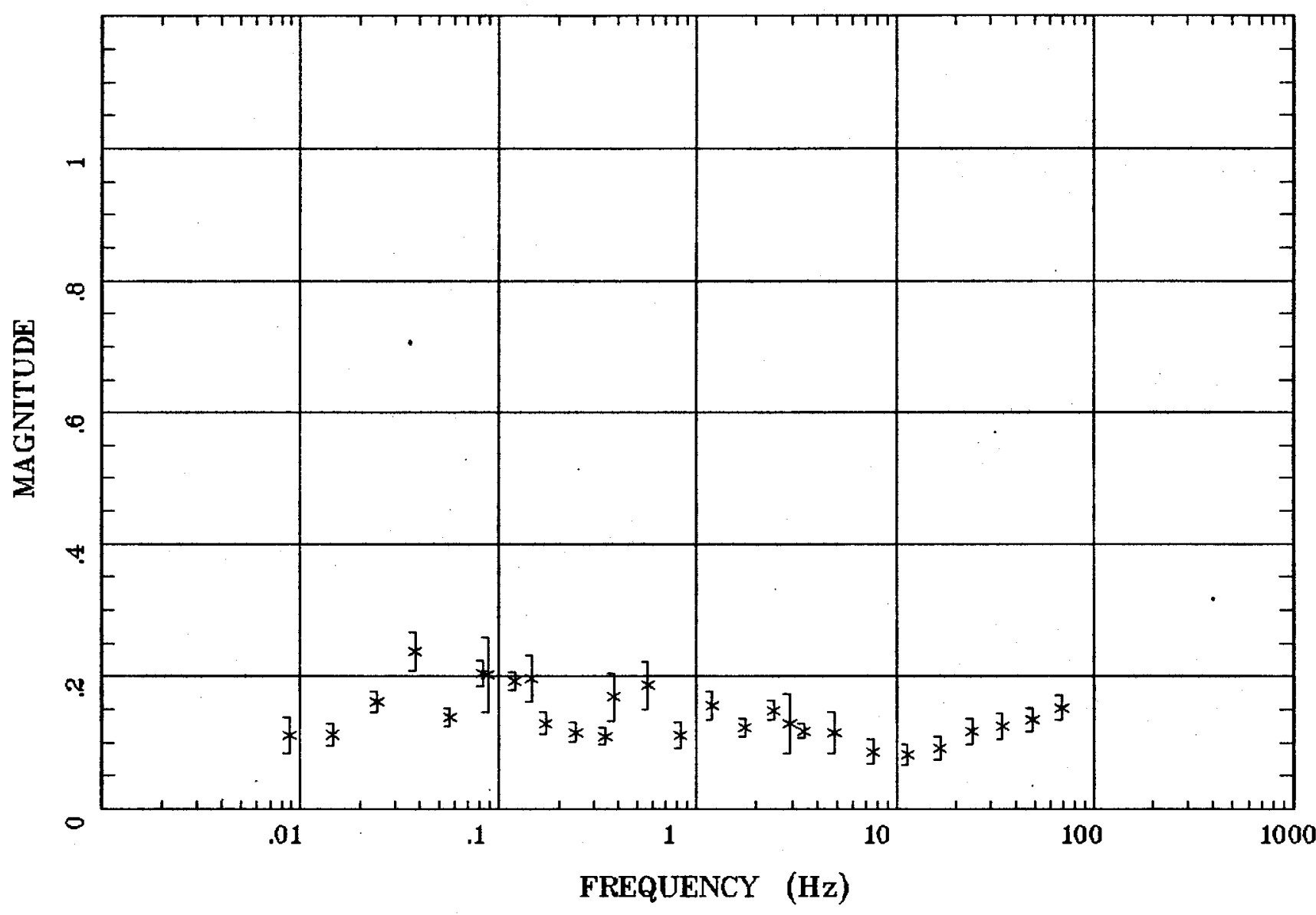

Client:

Remote: e-fld east $90 \mathrm{~m}$ Acquired: 10:3 Sep 09, 2003 Survey Co:USGS
Ratation:

Filename: ap12m.avg

Channels: Ch1 Ch2 Ch3 Ch4 Ch5 Ch6 Ch7 Plotted: 08:48 Sep 24, 2003

< EMI - ElectroMagnetic Instruments 


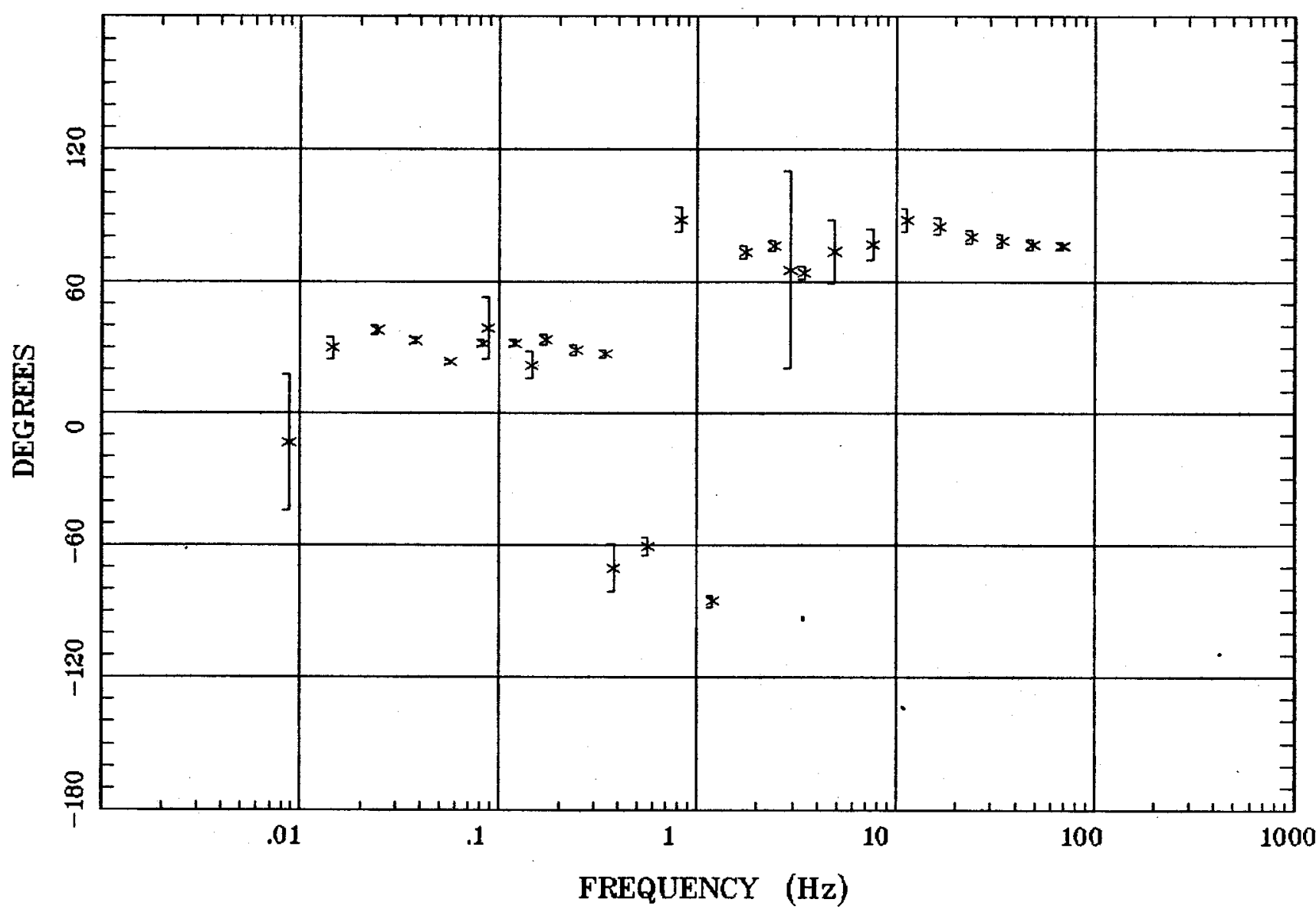

Client:

Remote: e-fld east $90 \mathrm{~m}$ Acquired: 10:3 Sep 09, 2003 Survey Co:USGS
Rotation:

Filename: ap12m.avg

Channels: Ch1 Ch2 Ch3 Ch4 Ch5 Ch6 Ch7 Plotted: 08:48 Sep 24, 2003

$<$ EMI - ElectroMagnetic Instruments 


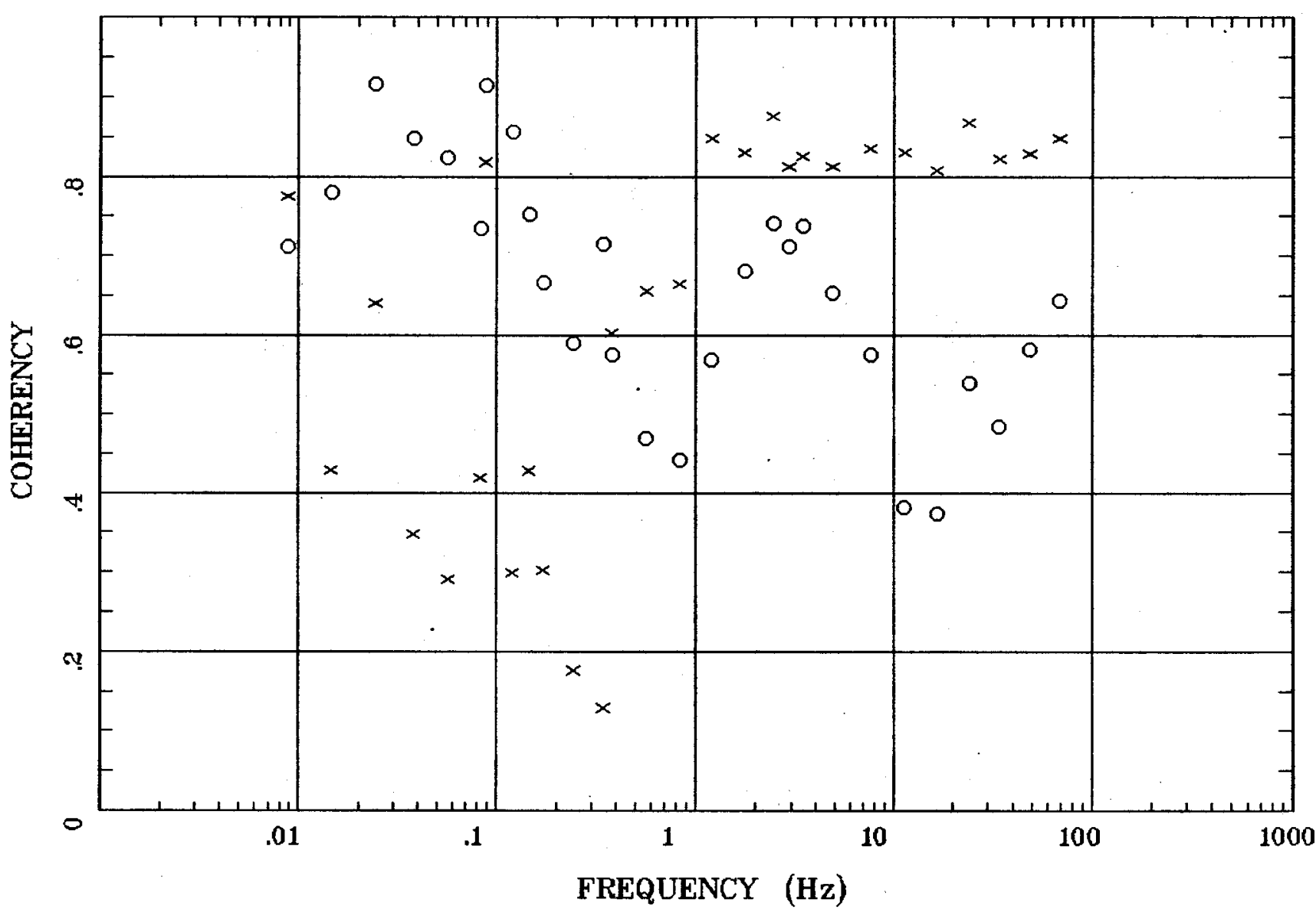

Client:

Remote: e-fld east $90 \mathrm{~m}$ Acquired: 10:3 Sep 09, 2003 Survey Co:USGS
Rotation:

Filename: ap12m.avg

Channels: Ch1 Ch2 Ch3 Ch4 Ch5 Ch6 Ch7 Plotted: 08:48 Sep 24, 2003

< EMI - ElectroMagnetic Instruments > 


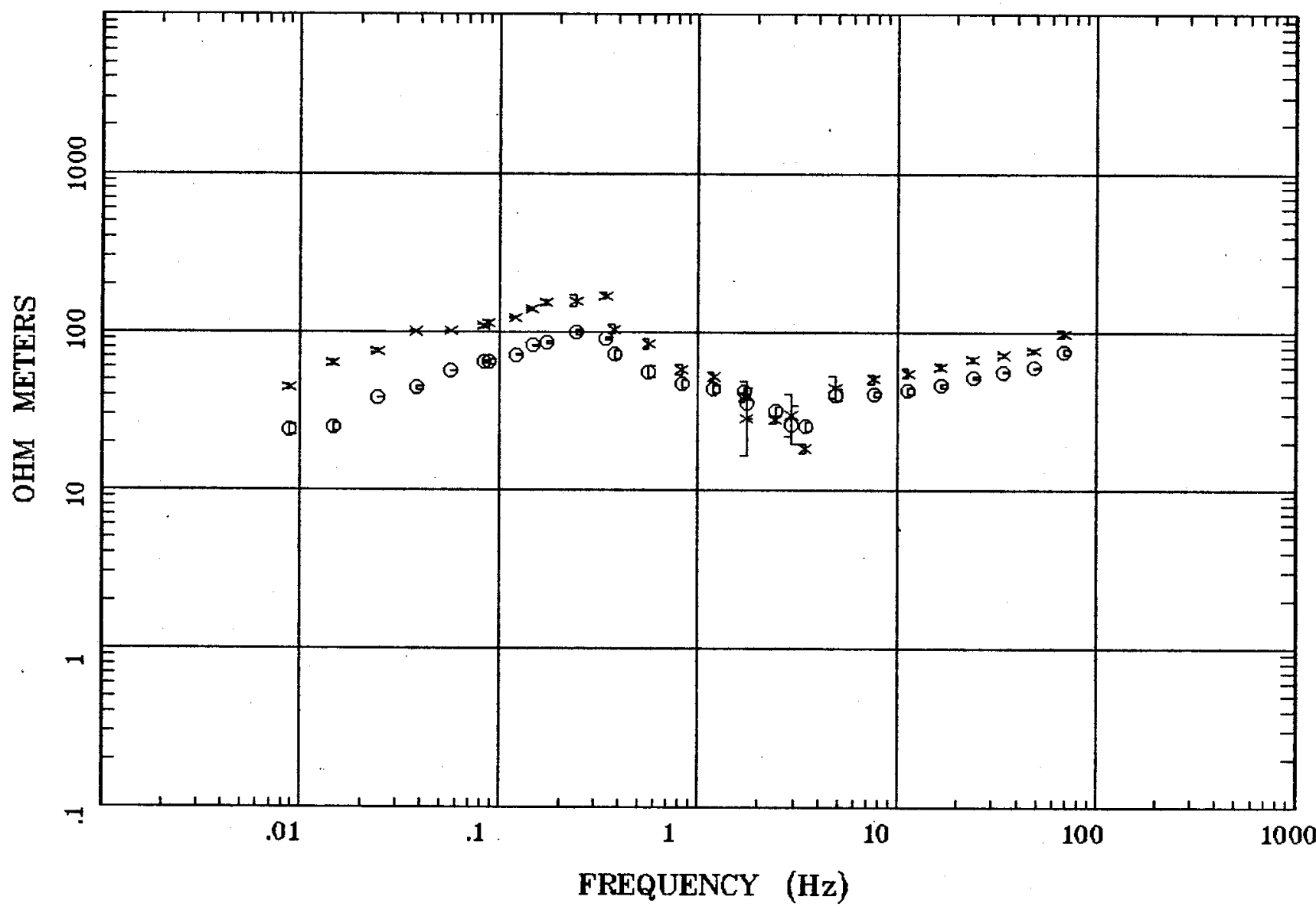

Client:

Remote: e-fld NE $90 \mathrm{~m}$

Acquired: 14:1 Sep 09, 2003 Survey Co:USGS
Rotation:

Filename: ap13mall.avg

Channels: Ch1 Ch2 Ch3 ch4 Ch5 Ch6 Ch7 Plotted: 08:49 Sep 24, 2003

$<$ EMI - ElectroMagnetic Instruments 


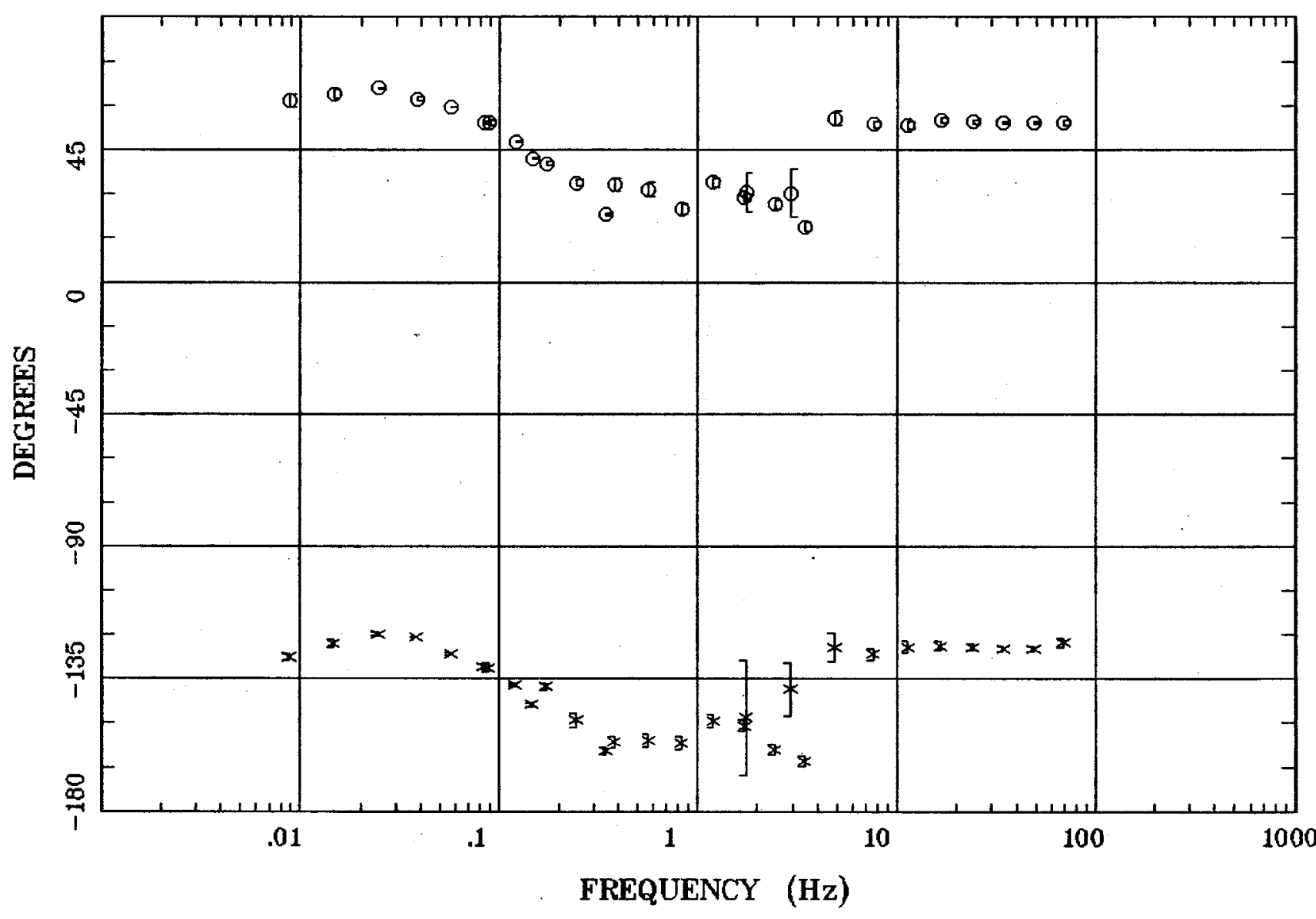

Client:

Remote: e-fld NE $90 \mathrm{~m}$ Acquired: 14:1 Sep 09, 2003 Survey Co:USGS
Rotation:

Filename: ap13mall.avg

Channels: Ch1 Ch2 Ch3 Ch4 Ch5 Ch6 Ch7 Plotted: 08:49 Sep 24, 2003

$<$ EMI - ElectroMagnetic Instruments 
Currie, NV $100 \mathrm{~K} \quad$ Station 13

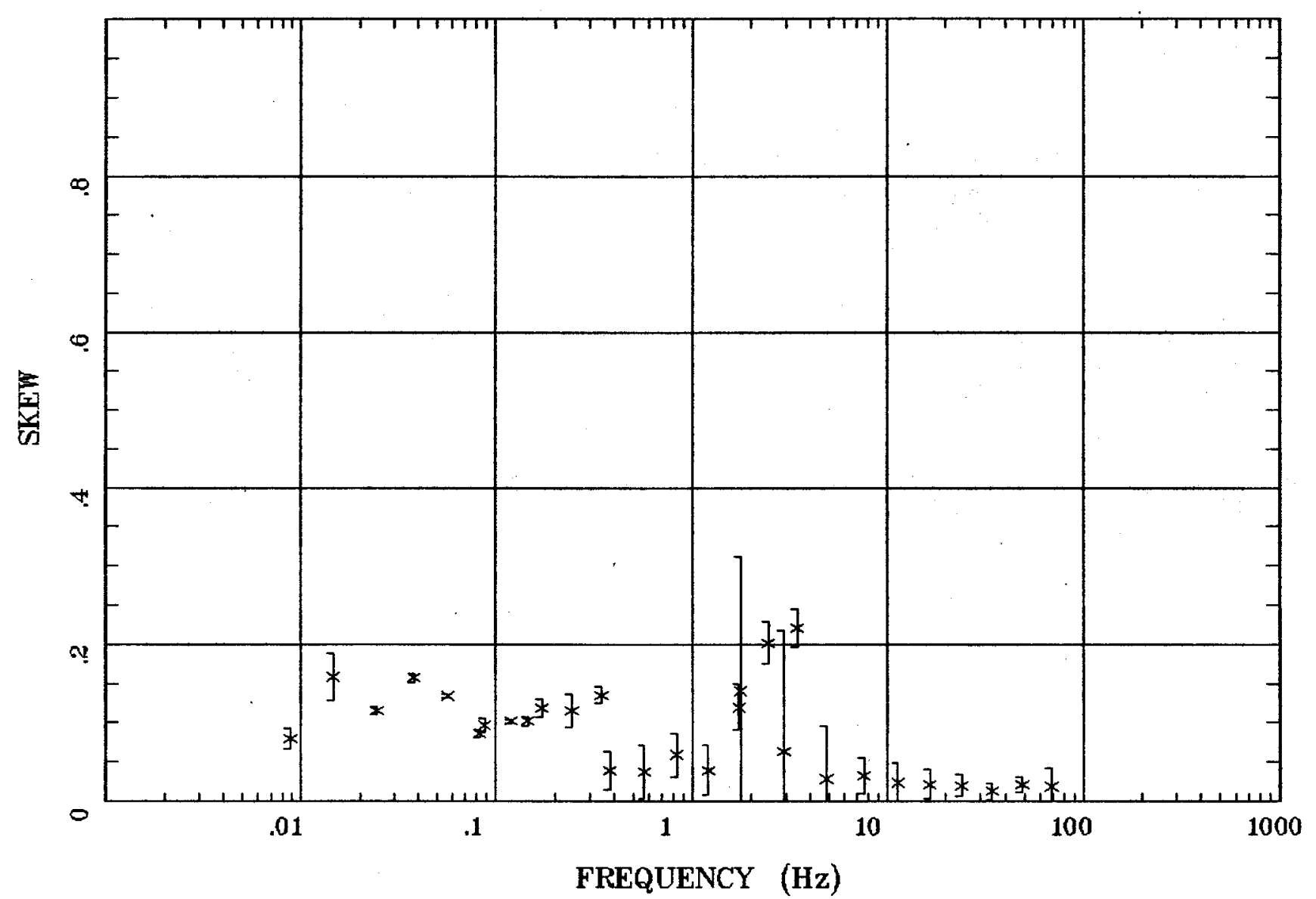

Client:

Remote: e-fld NE $90 \mathrm{~m}$

Acquired: 14:1 Sep 09, 2003

Survey Co:USGS
Rotation:

Filename: ap13mall.avg

Channels: Ch1 Ch2 Ch3 Ch4 Ch5 Ch6 Ch7

Plotted: 08:49 Sep 24, 2003

< EMI - ElectroMagnetic Instruments 


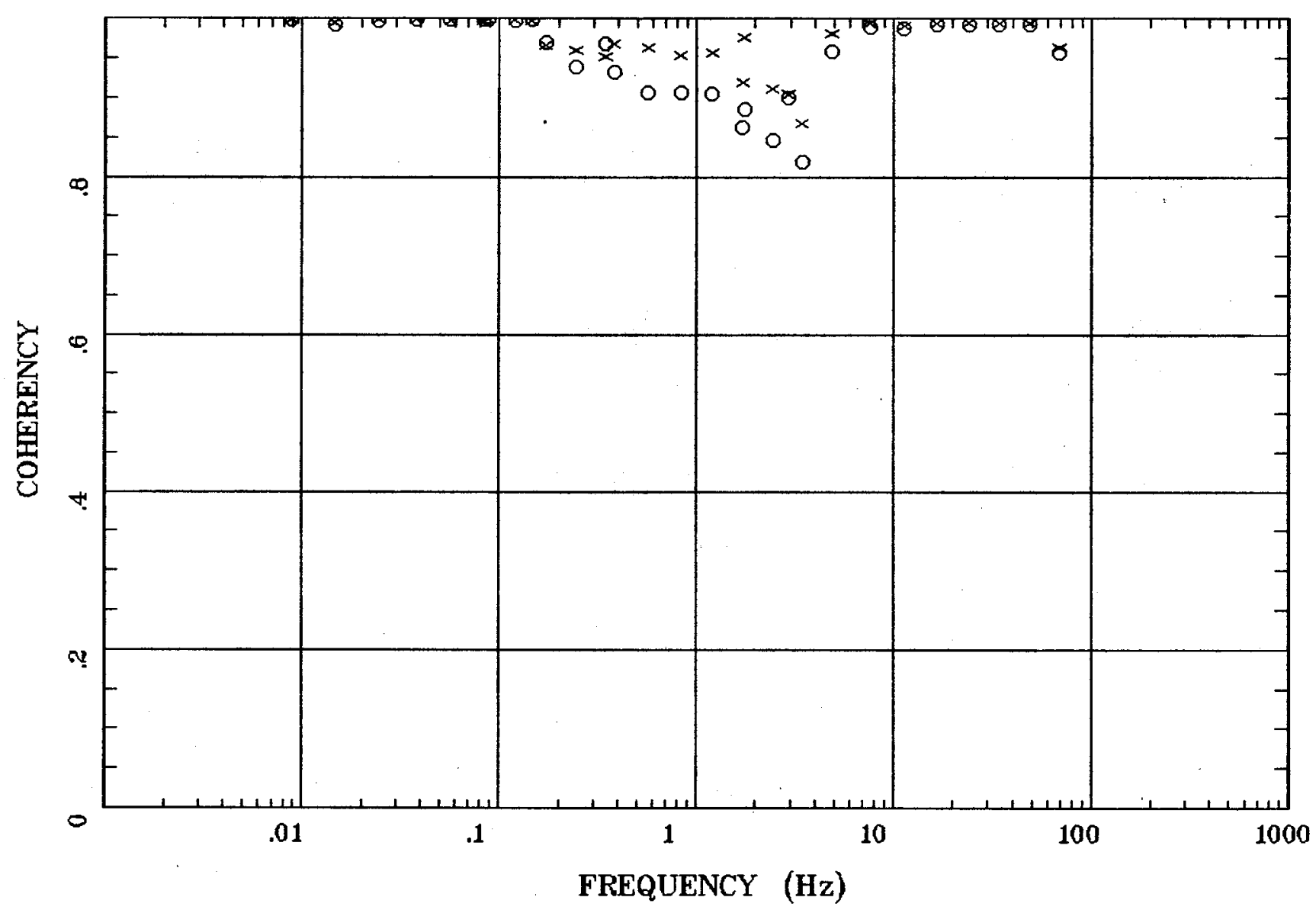

Client:

Remote: e-fld NE $90 \mathrm{~m}$

Acquired: 14:1 Sep 09, 2003

Survey Co:USGS
Rotation:

Filename: ap13mall.avg

Channels: Ch1 Ch2 Ch3 Ch4 Ch5 Ch6 Ch7

Plotted: 08:49 Sep 24, 2003

< EMI - ElectroMagnetic Instruments 


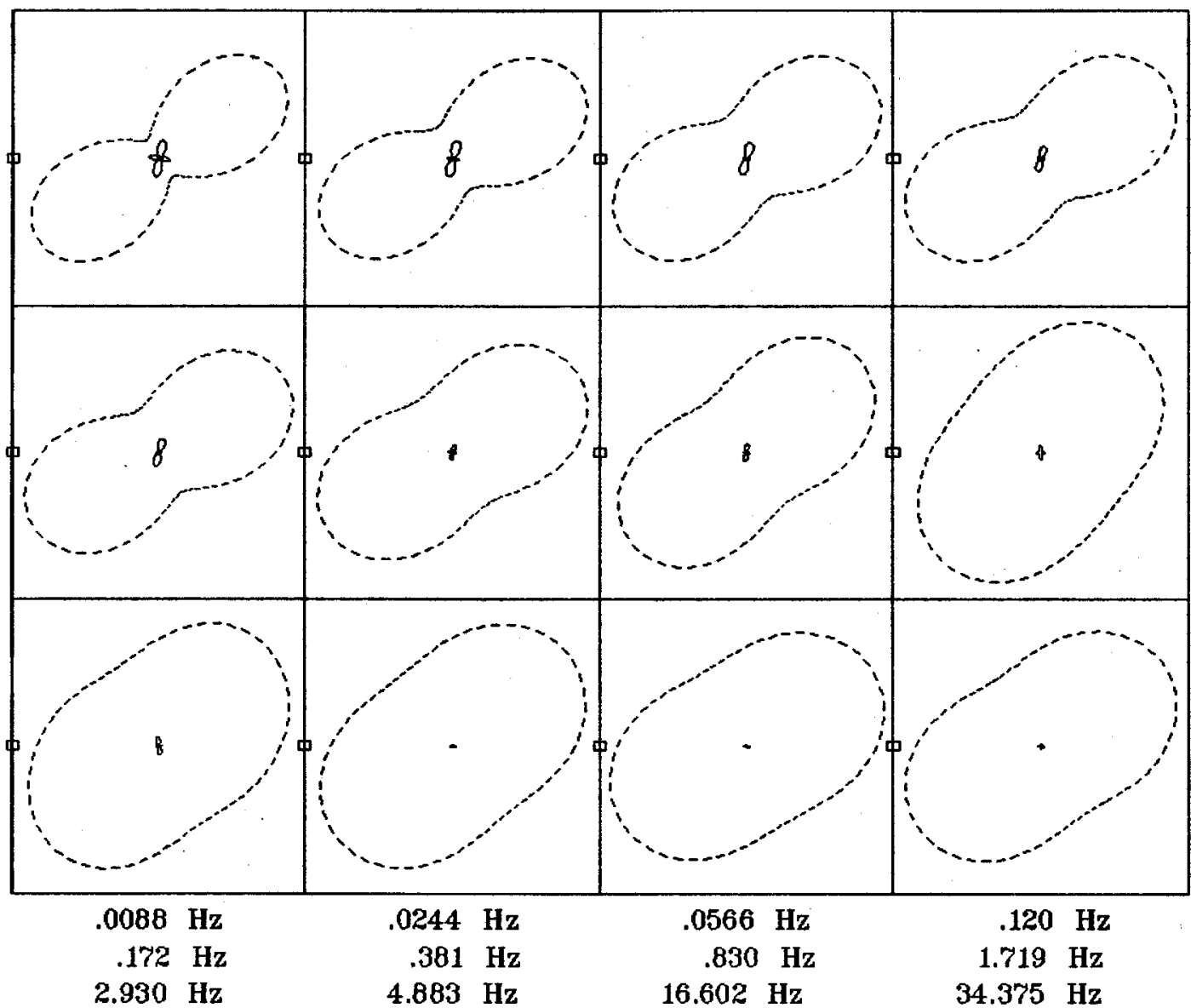

Client:

Remote: e-fld NE $90 \mathrm{~m}$

Acquired: 14:1 Sep 09, 2003 Survey Co:USGS
Rotation:

Filename: ap13mall.avg Channels: Ch1 Ch2 Ch3 Ch4 Ch5 Ch6 Ch7 Plotted: 08:49 Sep 24, 2003

< EMI - ElectroMagnetic Instruments 


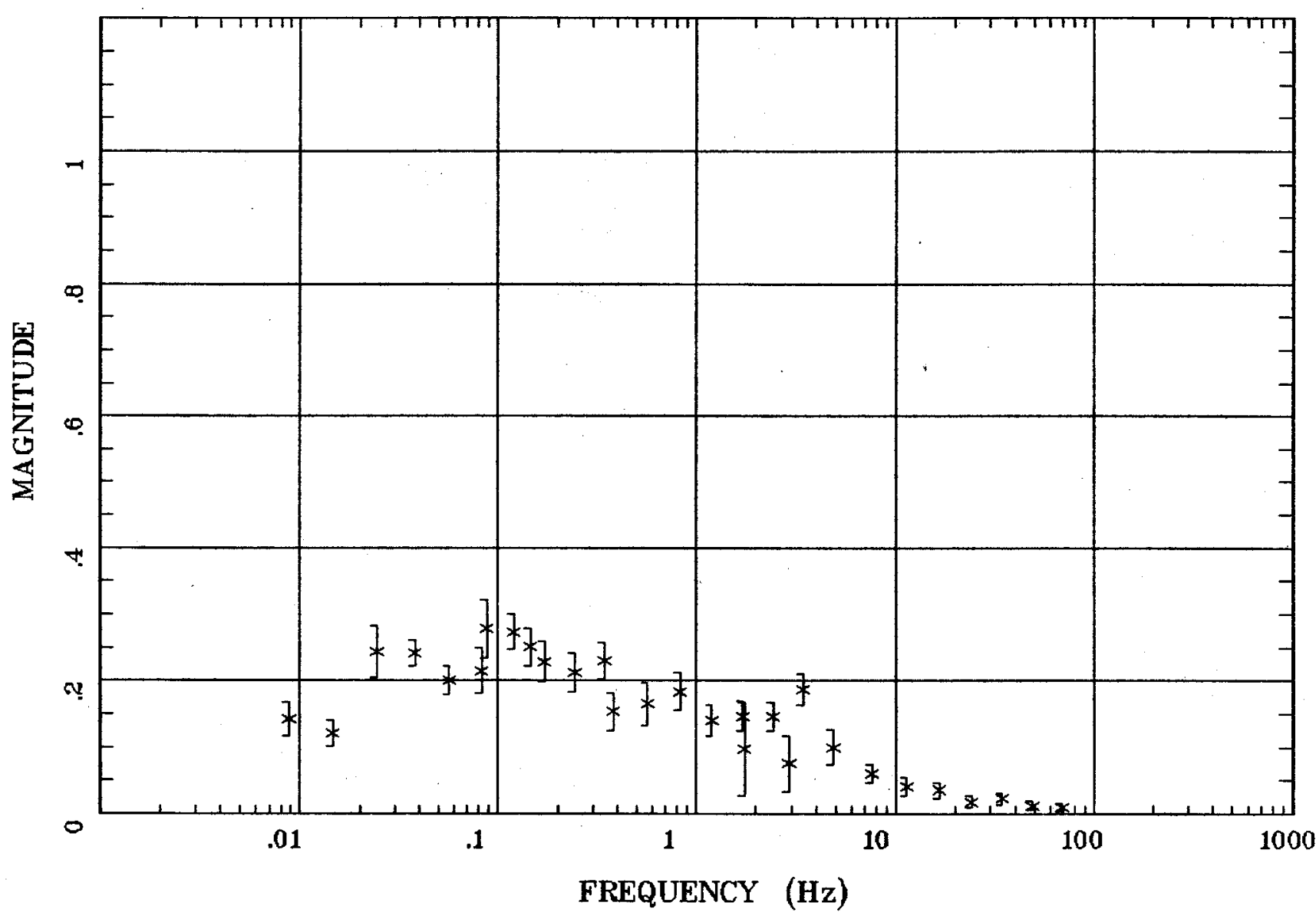

Client:

Remote: e-fld NE $90 \mathrm{~m}$

Acquired: 14:1 Sep 09, 2003

Survey Co:USGS
Rotation:

Filename: ap13mall.avg

Channels: Ch1 Ch2 Ch3 Ch4 Ch5 Ch6 Ch7 Plotted: 08:49 Sep 24, 2003

$<$ EMI - ElectroMagnetic Instruments 


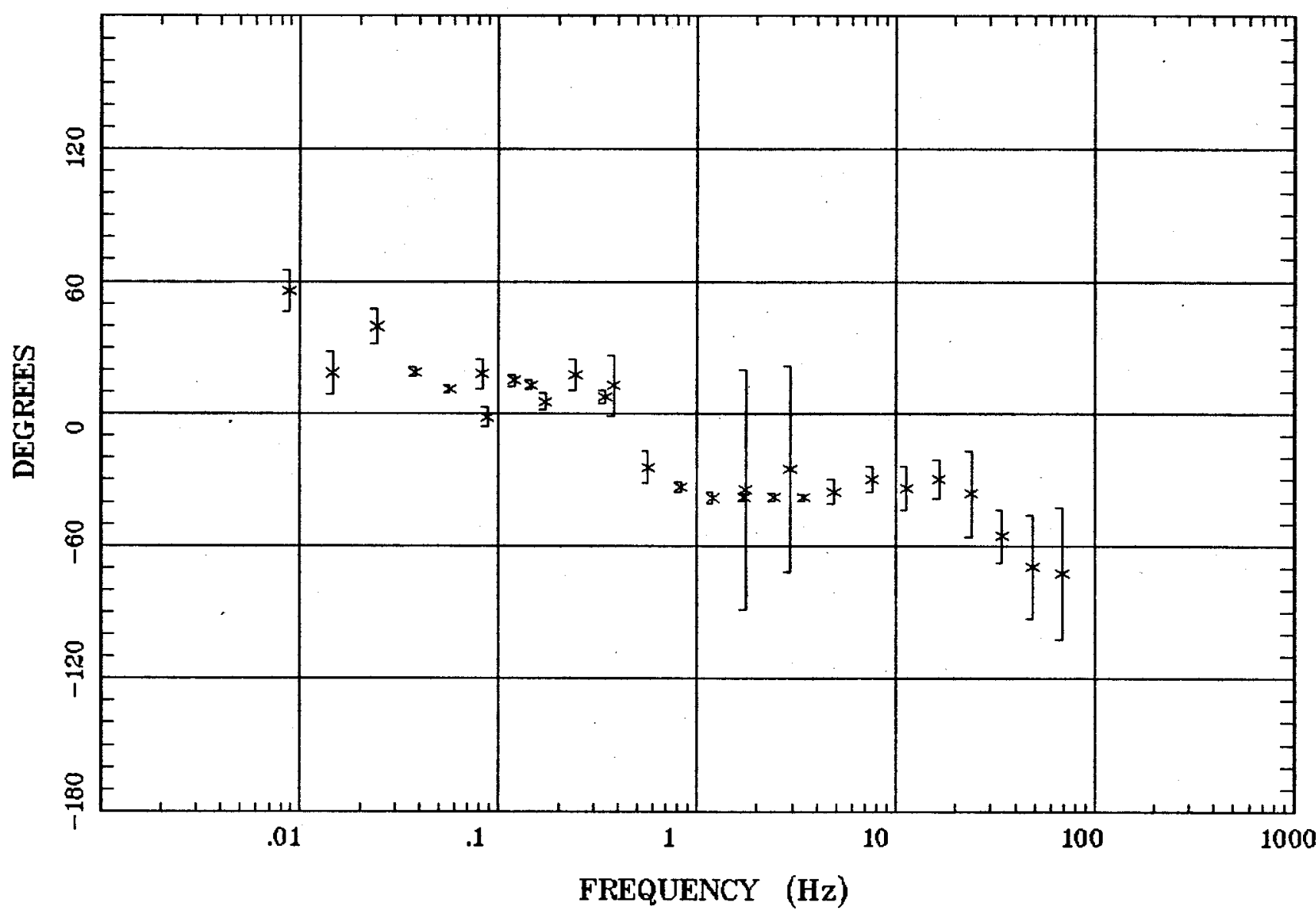

Client:

Remote: e-fld NE $90 \mathrm{~m}$

Acquired: 14:1 Sep 09, 2003

Survey Co:USGS
Rotation:

Filename: ap13mall.avg

Channels: Ch1 Ch2 Ch3 Ch4 Ch5 Ch6 Ch7

Plotted: 08:49 Sep 24, 2003

< EMI - ElectroMagnetic Instruments 
HzHx.x Coh HzHy.o

Currie, NV $100 \mathrm{~K}$

Station 13

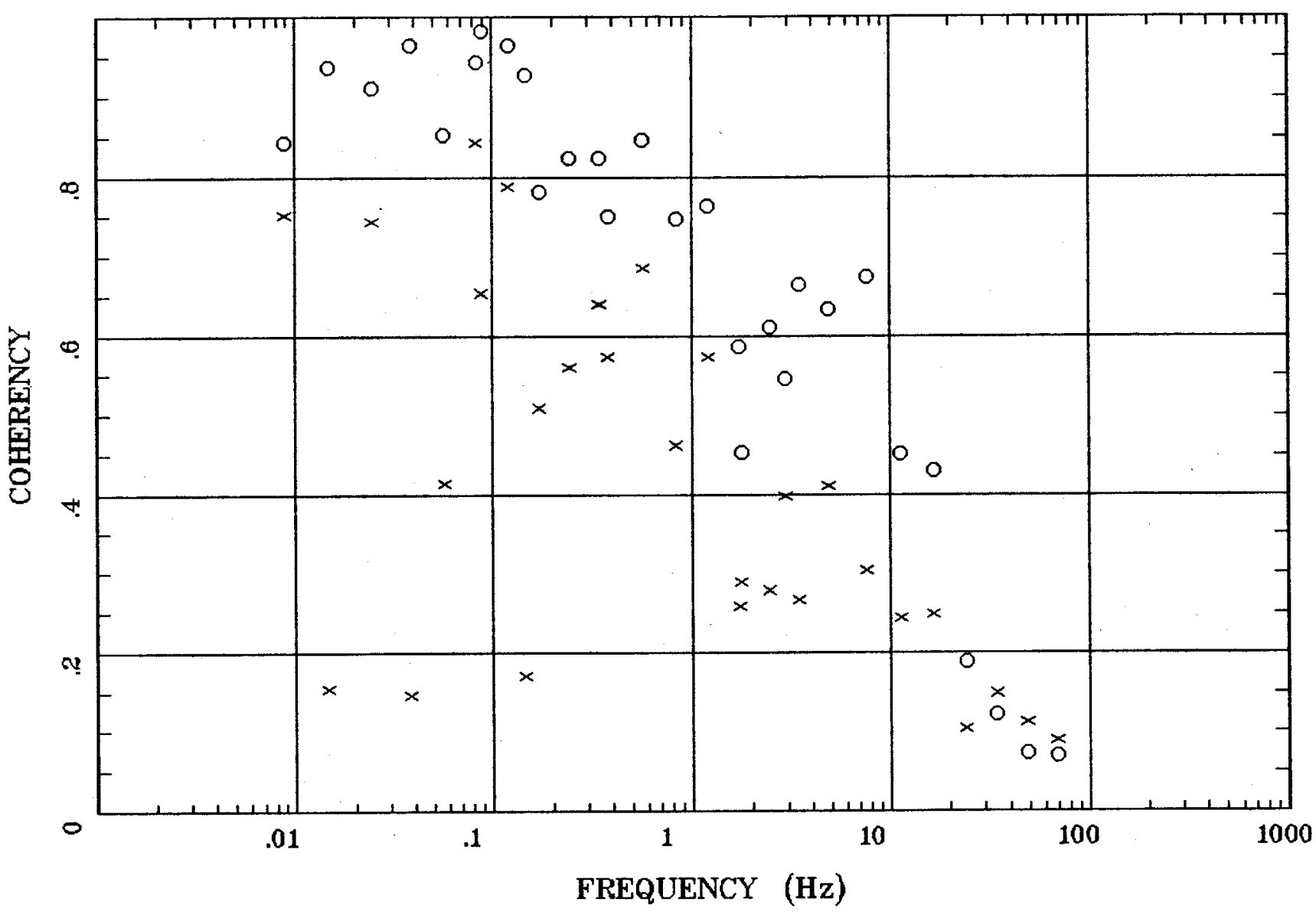

Client:

Remote: e-fld NE $90 \mathrm{~m}$

Acquired: $14: 1$ Sep 09, 2003 Survey Co:USGS
Rotation:

Filename: ap13mall.avg

Channels: Ch1 Ch2 Ch3 Ch4 Ch5 Ch6 Ch7 Plotted: 08:49 Sep 24, 2003

$<$ EMI - ElectroMagnetic Instruments 


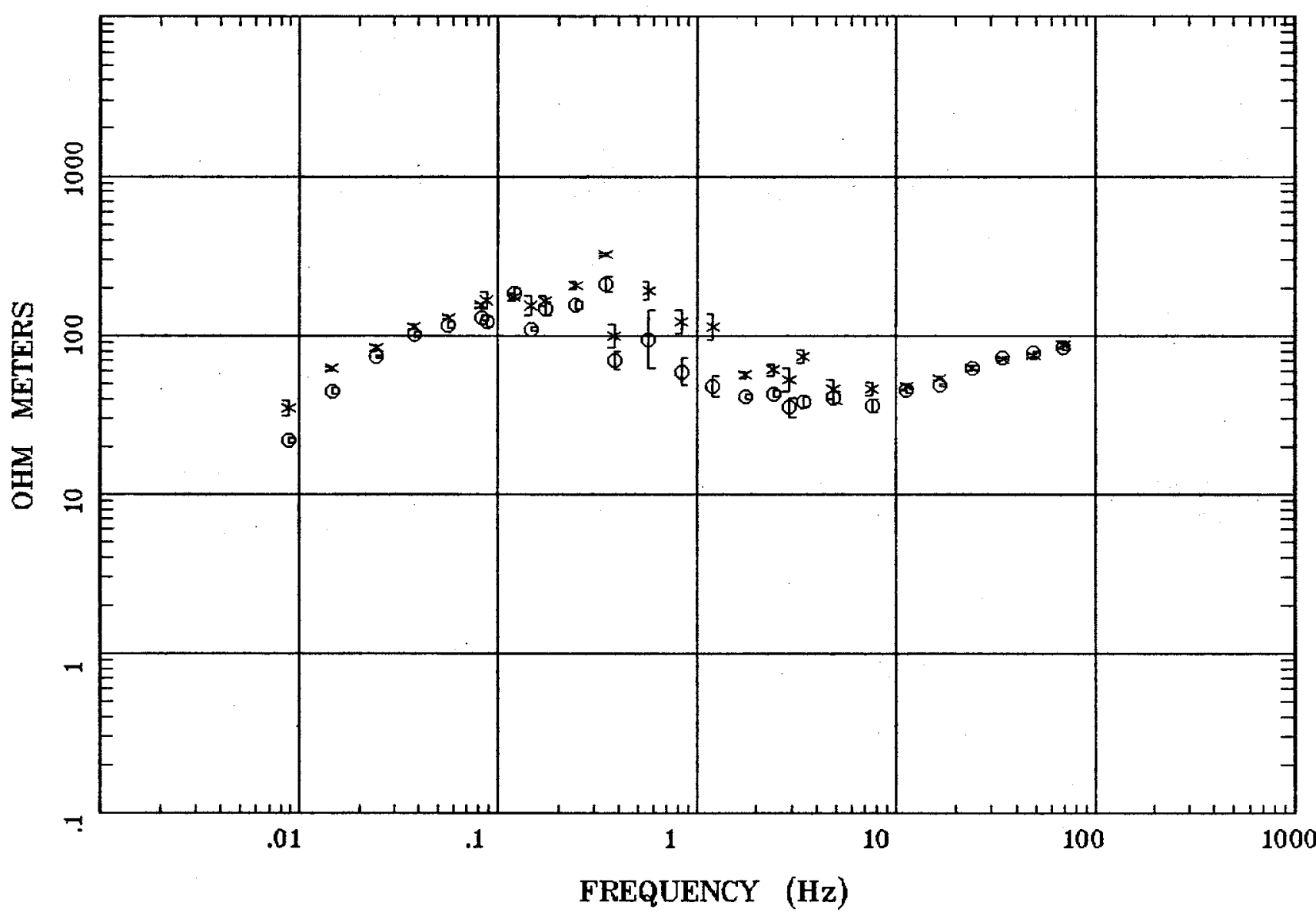

Client:

Remote: e-fld SE $90 \mathrm{~m}$

Acquired: 10:5 Sep 10, 2003

Survey Co:USGS
Rotation:

Filename: ap14mall.avg

Channels: Ch1 Ch2 Ch3 Ch4 Ch5 Ch6 Ch7 Plotted: 08:49 Sep 24, 2003

< EMI - ElectroMagnetic Instruments 
Kern Mts., NV 100k Station 14

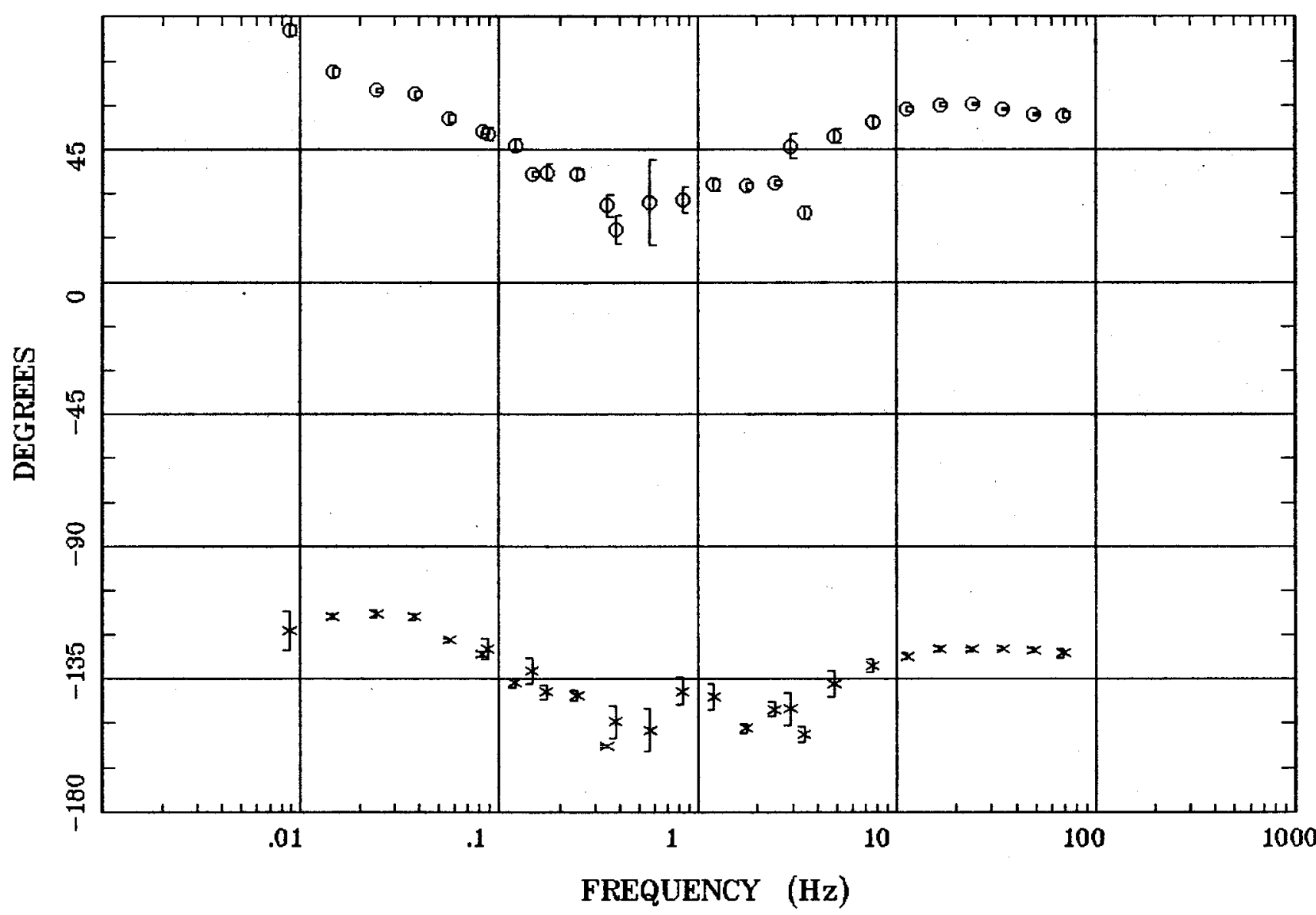

Client:

Remote: e-fld SE $90 \mathrm{~m}$

Acquired: 10:5 Sep 10, 2003

Survey Co:USGS
Rotation:

Filename: ap14mall.avg

Channels: Ch1 Ch2 Ch3 Ch4 Ch5 Ch6 Ch7

Plotted: 08:50 Sep 24, 2003

$<$ EMI - ElectroMagnetic Instruments 


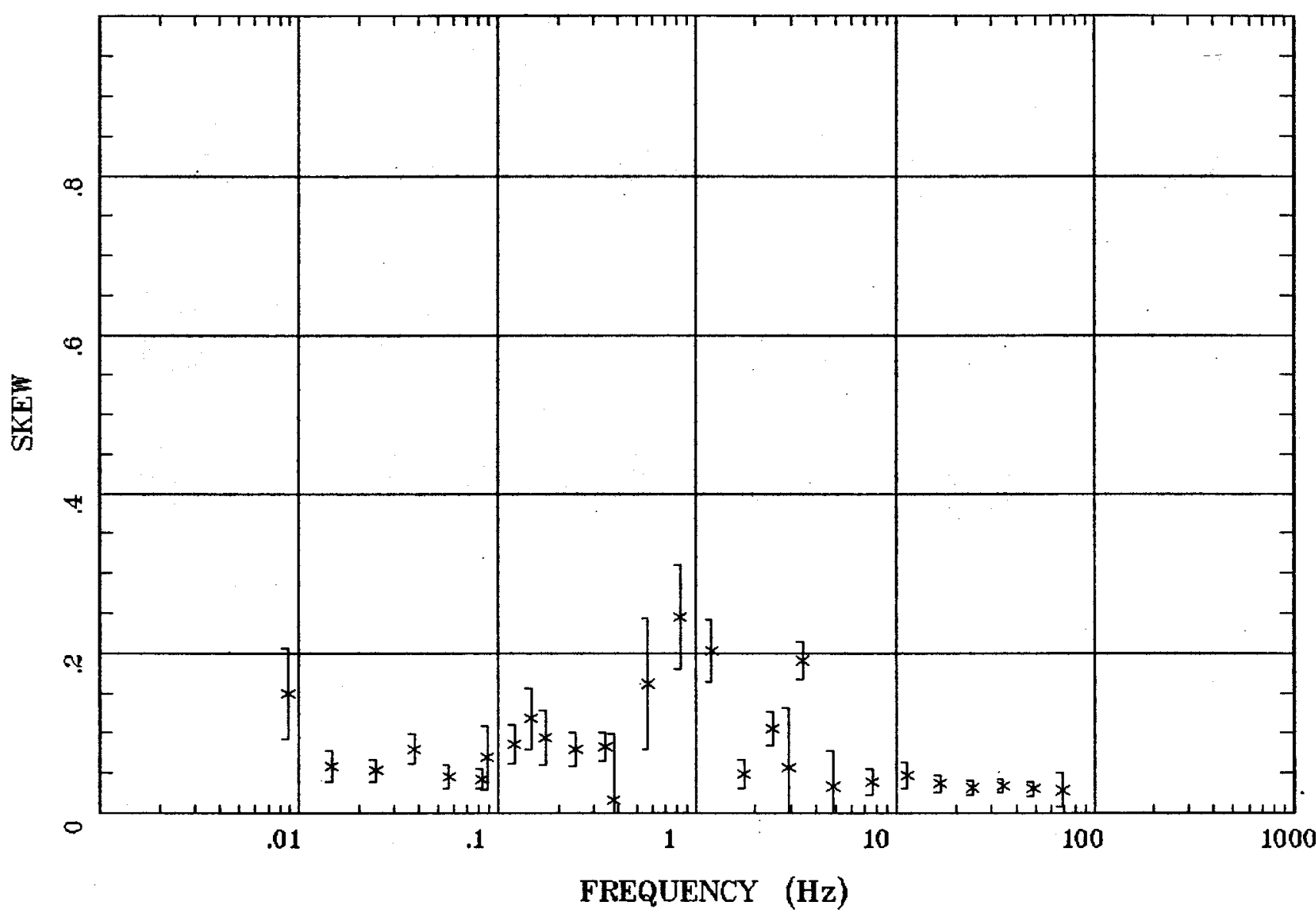

Client:

Remote: e-fld SE $90 \mathrm{~m}$

Acquired: 10:5 Sep 10, 2003

Survey Co:USGS
Rotation:

Filename: ap14mall.avg

Channels: Ch1 Ch2 Ch3 Ch4 Ch5 Ch6 Ch7

Plotted: 08:50 Sep 24, 2003

$<$ EMI - ElectroMagnetic Instruments > 
E MULT Coh.

Kern Mts., NV 100k Station 14

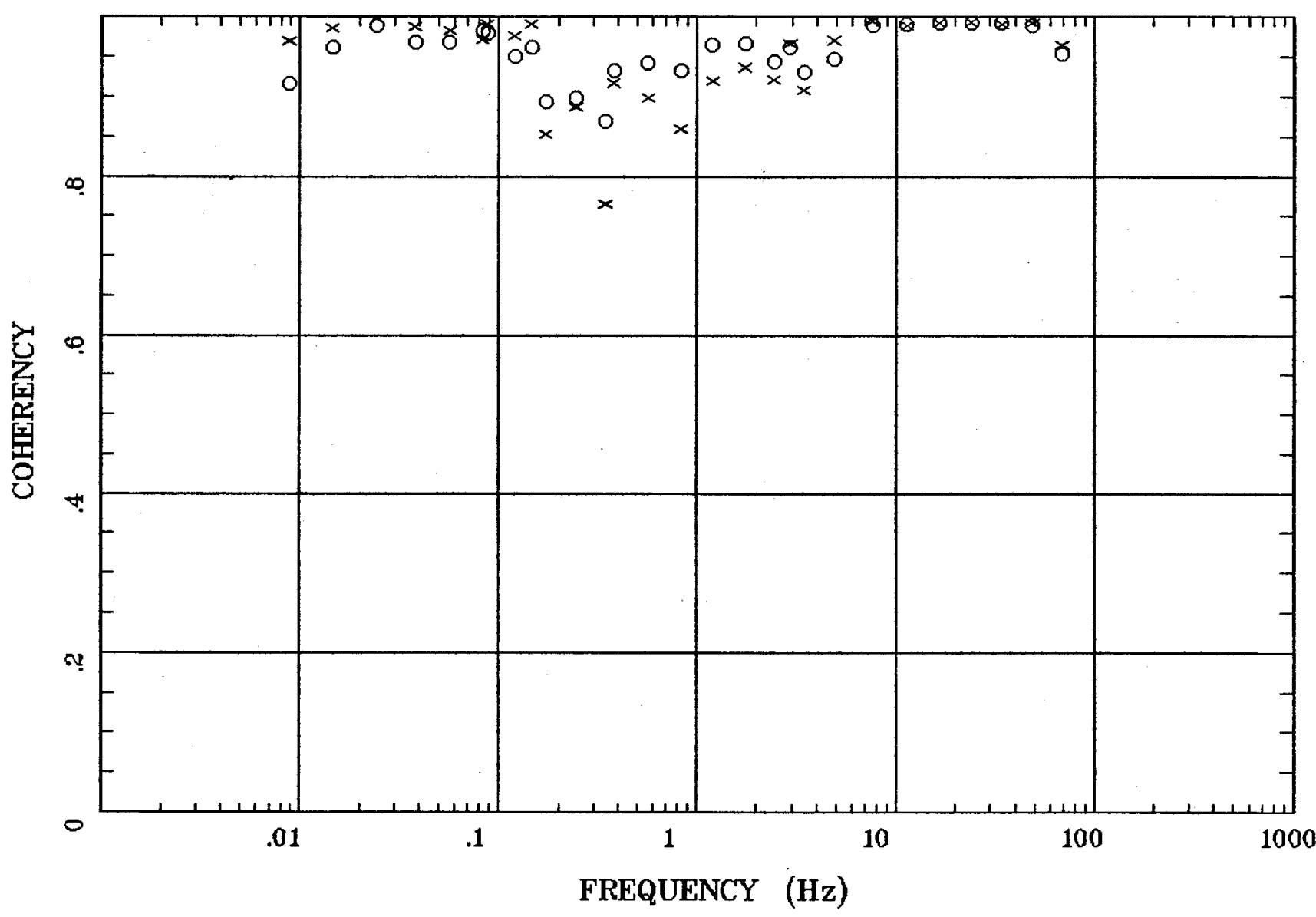

Client:

Remote: e-fld SE $90 \mathrm{~m}$

Acquired: 10:5 Sep 10, 2003

Survey Co:USGS
Rotation:

Filename: ap14mall.avg

Channels: Ch1 Ch2 Ch3 Ch4 Ch5 Ch6 Ch7

Plotted: 08:50 Sep 24, 2003

<EMI - ElectroMagnetic Instruments > 


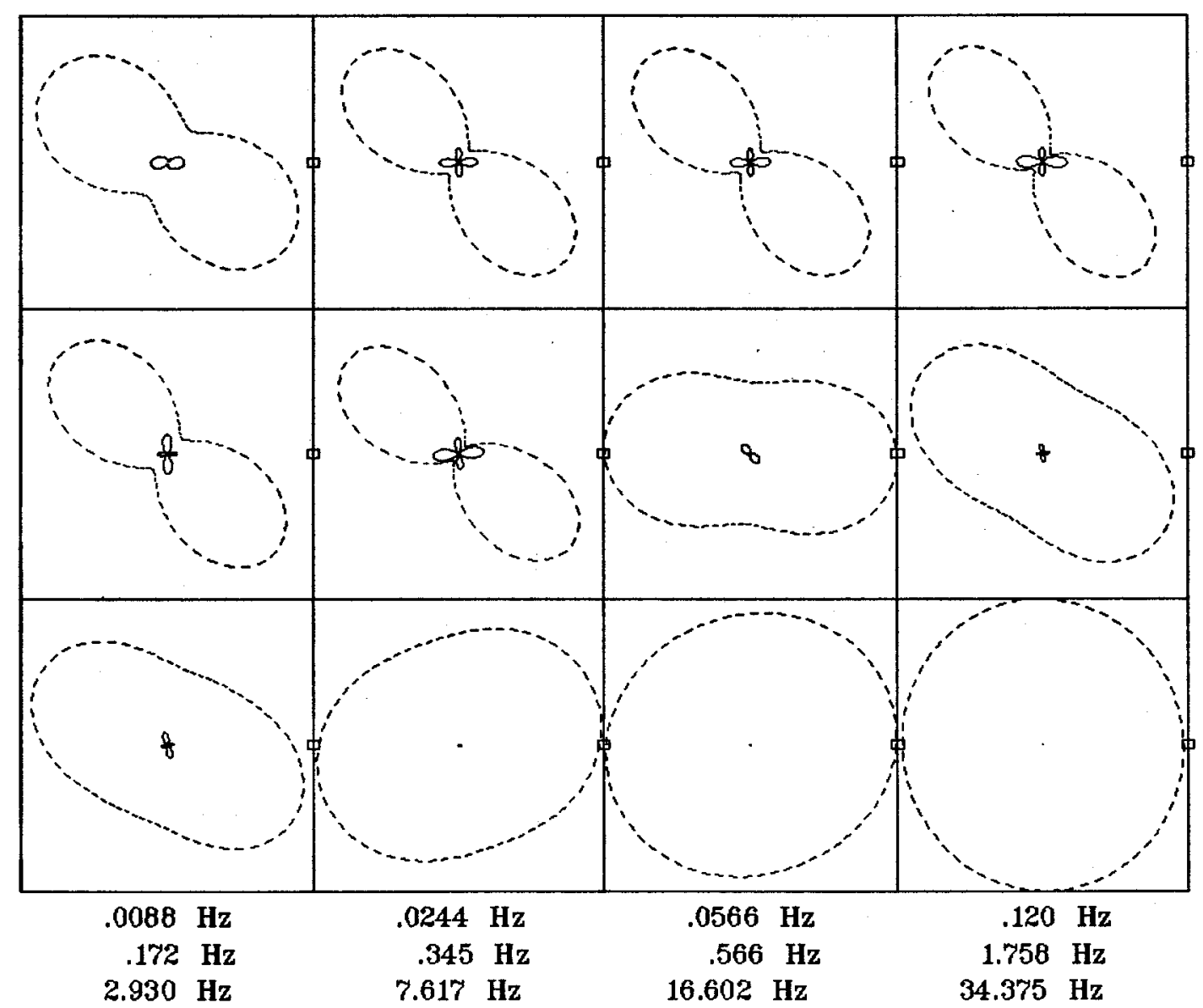

\section{Client:}

Remote: e-fld SE $90 \mathrm{~m}$ Acquired: 10:5 Sep 10, 2003 Survey Co:USGS

\section{Rotation:}

Filename: ap14mall.avg

Channels: Ch1 Ch2 Ch3 Ch4 Ch5 Ch6 Ch7

Plotted: 08:50 Sep 24, 2003

$<$ EMI - ElectroMagnetic Instruments $>$ 


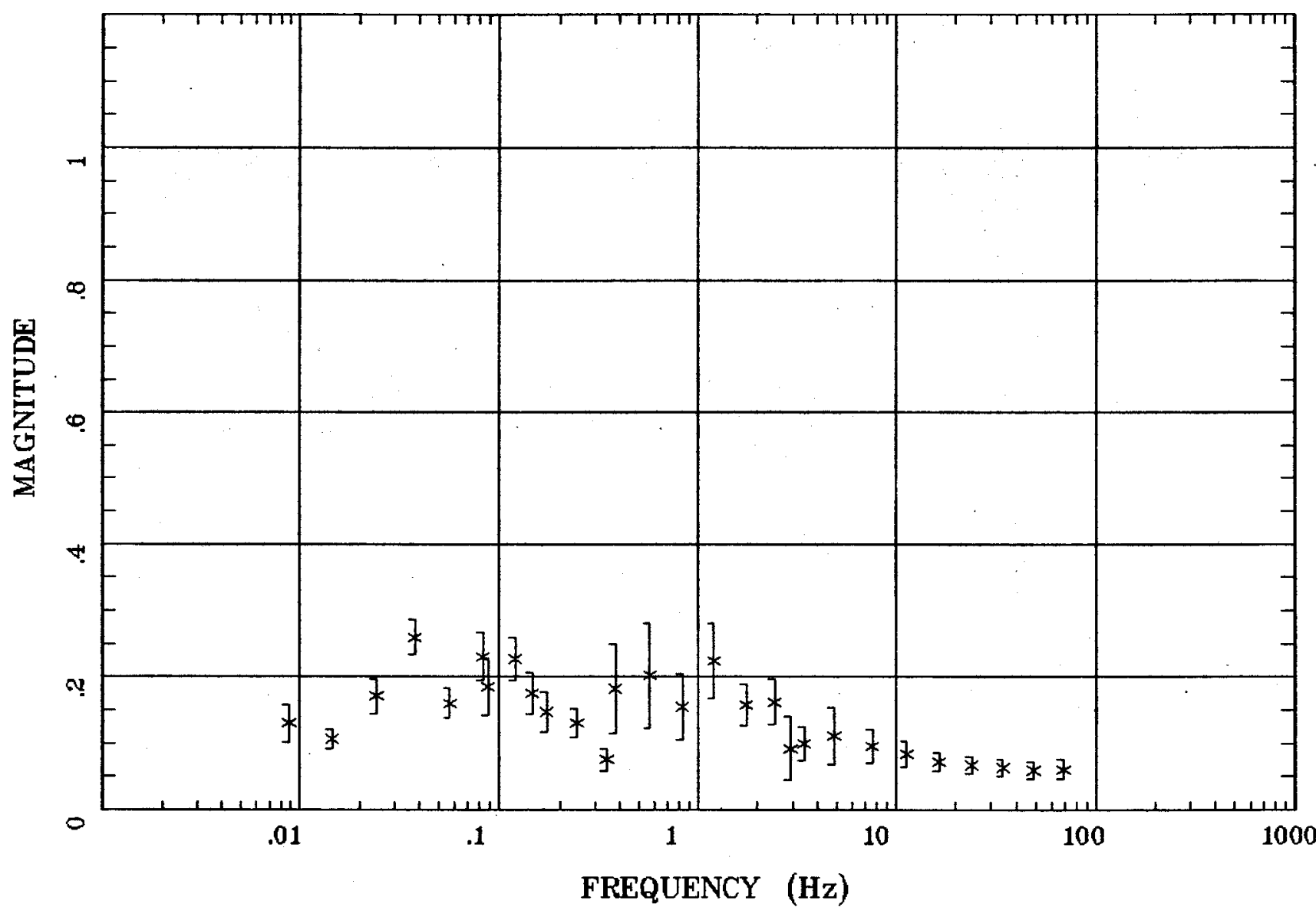

Client:

Remote: e-fld SE $90 \mathrm{~m}$

Acquired: 10:5 Sep 10, 2003

Survey Co:USGS
Rotation:

Filename: ap14mall.avg

Channels: Ch1 Ch2 Ch3 Ch4 Ch5 Ch6 Ch7

Plotted: 08:50 Sep 24, 2003

$<$ EMI - ElectroMagnetic Instruments 


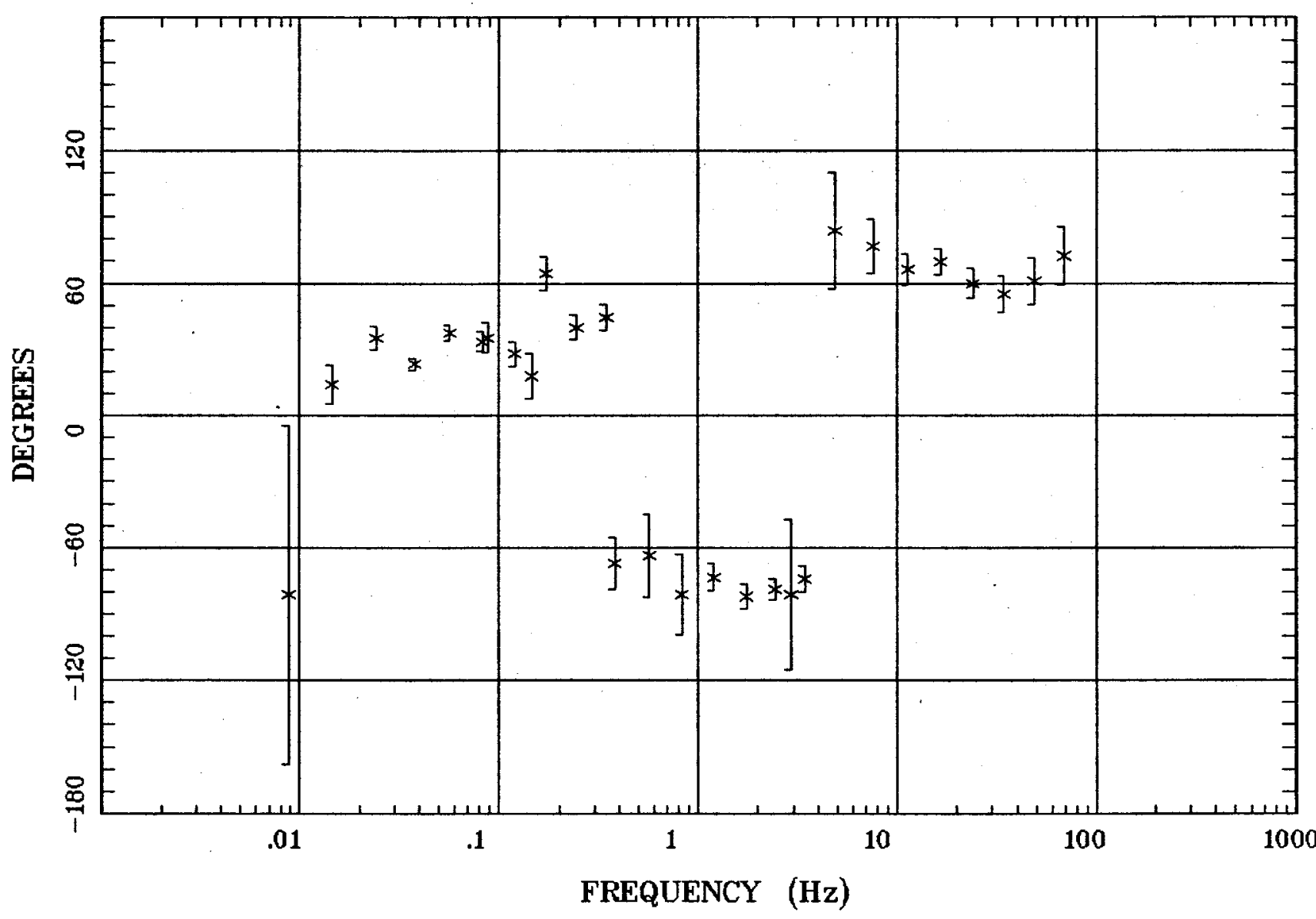

Client:

Remote: e-fld SE $90 \mathrm{~m}$

Acquired: 10:5 Sep 10, 2003 Survey Co:USGS
Rotation:

Filename: ap14mall.avg

Channels: Ch1 Ch2 Ch3 Ch4 Ch5 Ch6 Ch7 Plotted: 08:50 Sep 24, 2003

$<$ EMI - ElectroMagnetic Instruments 
HzHx.x Coh HzHy.o

Kern Mts., NV 100k Station 14

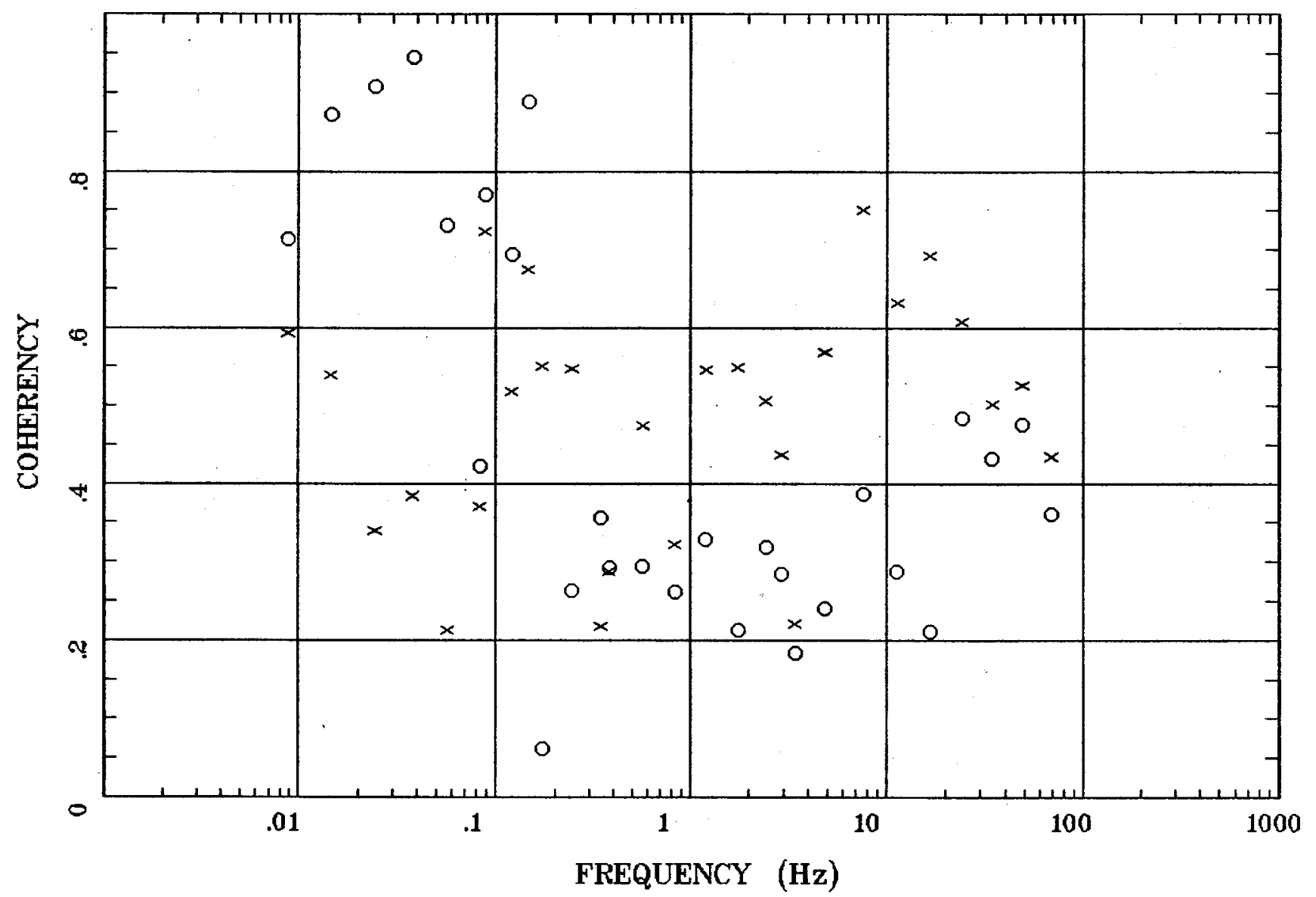

Client:

Remote: e-fld SE $90 \mathrm{~m}$ Acquired: 10:5 Sep 10, 2003 Survey Co:USGS
Rotation:

Filename: ap14mall.avg

Channels: Ch1 Ch2 Ch3 Ch4 Ch5 Ch6 Ch7 Plotted: 08:50 Sep 24, 2003

< EMI - ElectroMagnetic Instruments . 


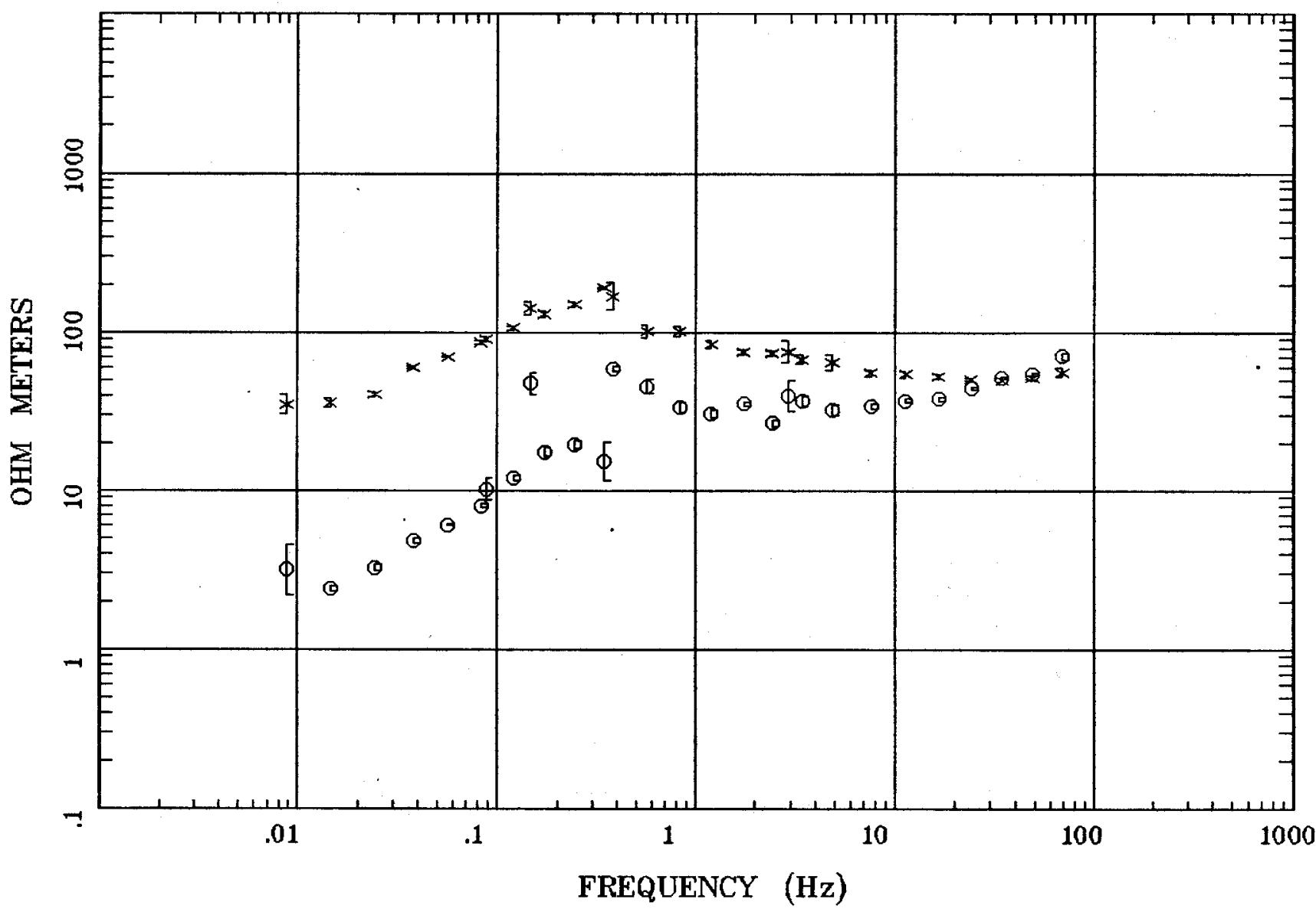

Client:

Remote: e-fld E $90 \mathrm{~m}$

Acquired: 14:2 Sep 10, 2003 Survey Co:USGS
Rotation:

Filename: ap $15 \mathrm{~m} . \mathrm{avg}$

Channels: Ch6 Ch7 Ch3 Ch4 Ch5 Ch6 Ch7 Plotted: 14:29 Sep 24, 2003

< EMI - ElectroMagnetic Instruments 


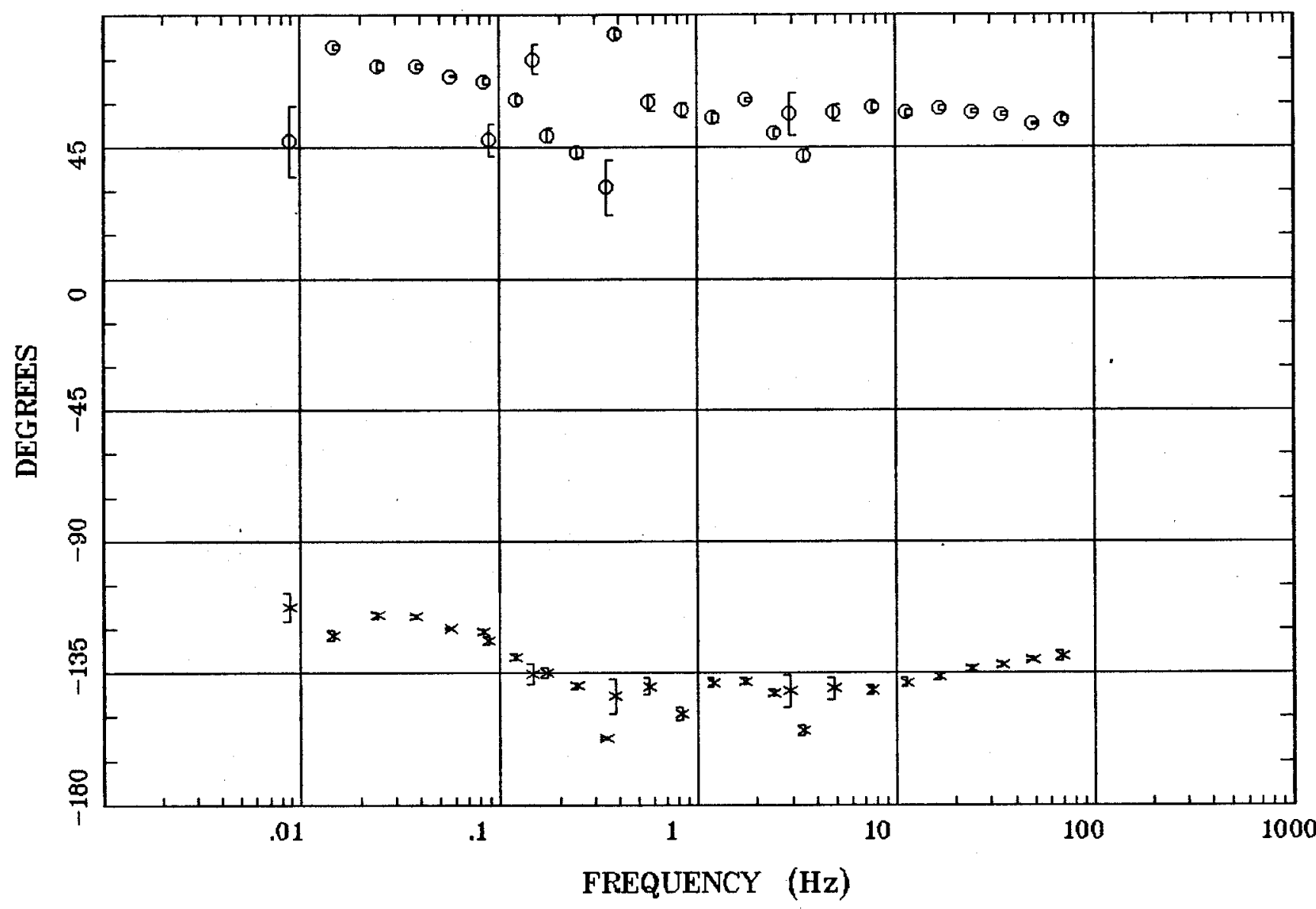

Client:

Remote: e-fld E $90 \mathrm{~m}$

Acquired: 14:2 Sep 10, 2003

Survey Co:USGS
Rotation:

Filename: ap $15 \mathrm{~m}$.avg

Channels: Ch6 Ch' Ch3 Ch4 Ch5 Ch6 Ch7

Platted: 14:29 Sep 24, 2003

$<$ EMI - ElectroMagnetic Instruments > 


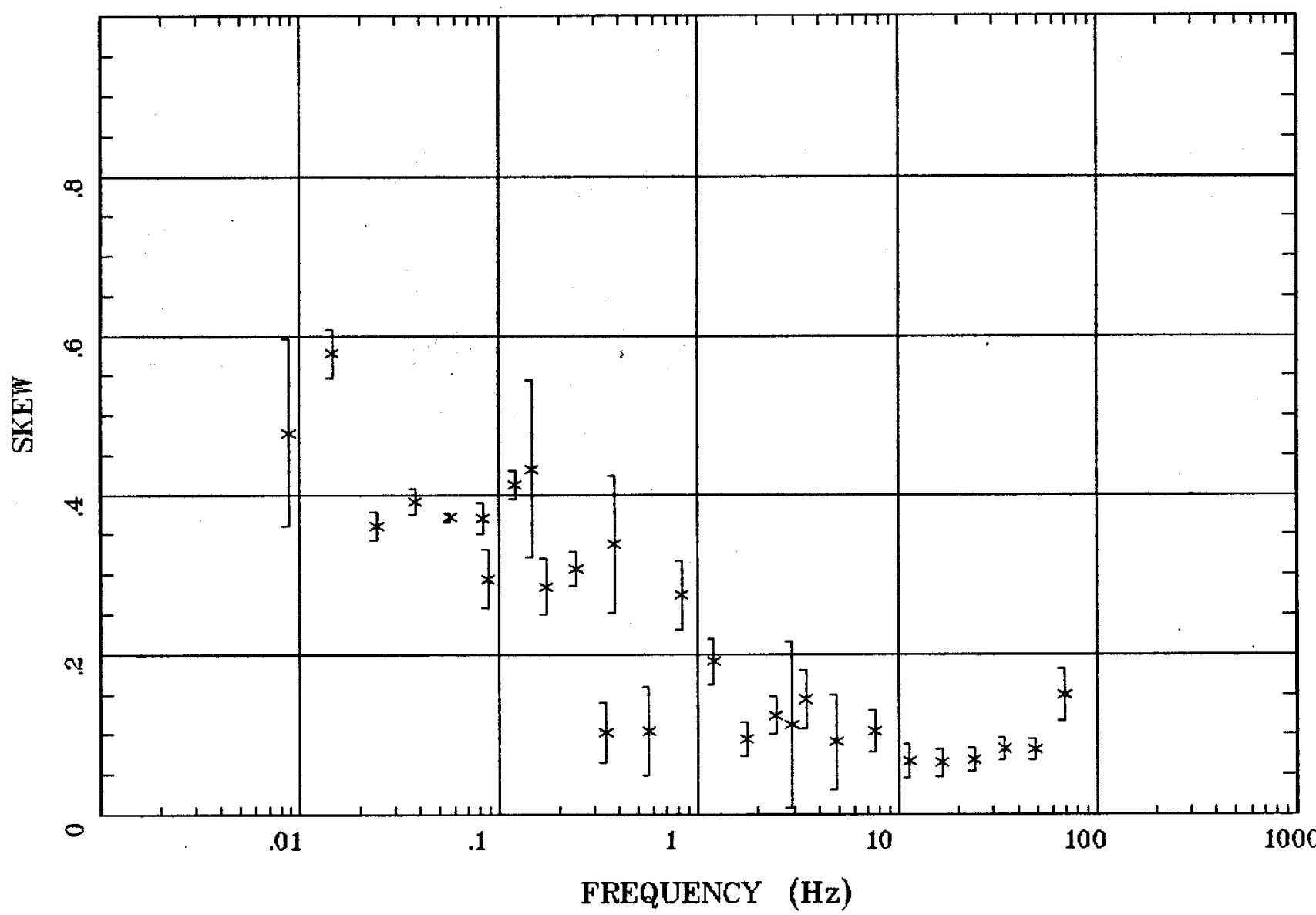

Client:

Remote: e-fld E 90 m

Acquired: 14:2 Sep 10, 2003 Survey Co:USGS
Rotation:

Filename: ap15m.avg

Channels: Ch6 Ch7 Ch3 ch4 Ch5 Ch6 Ch7 Plotted: 14:29 Sep 24, 2003

$<$ EMI - ElectroMagnetic Instruments 
E MULT Coh.

Kern Mts., NV 100k Station 15

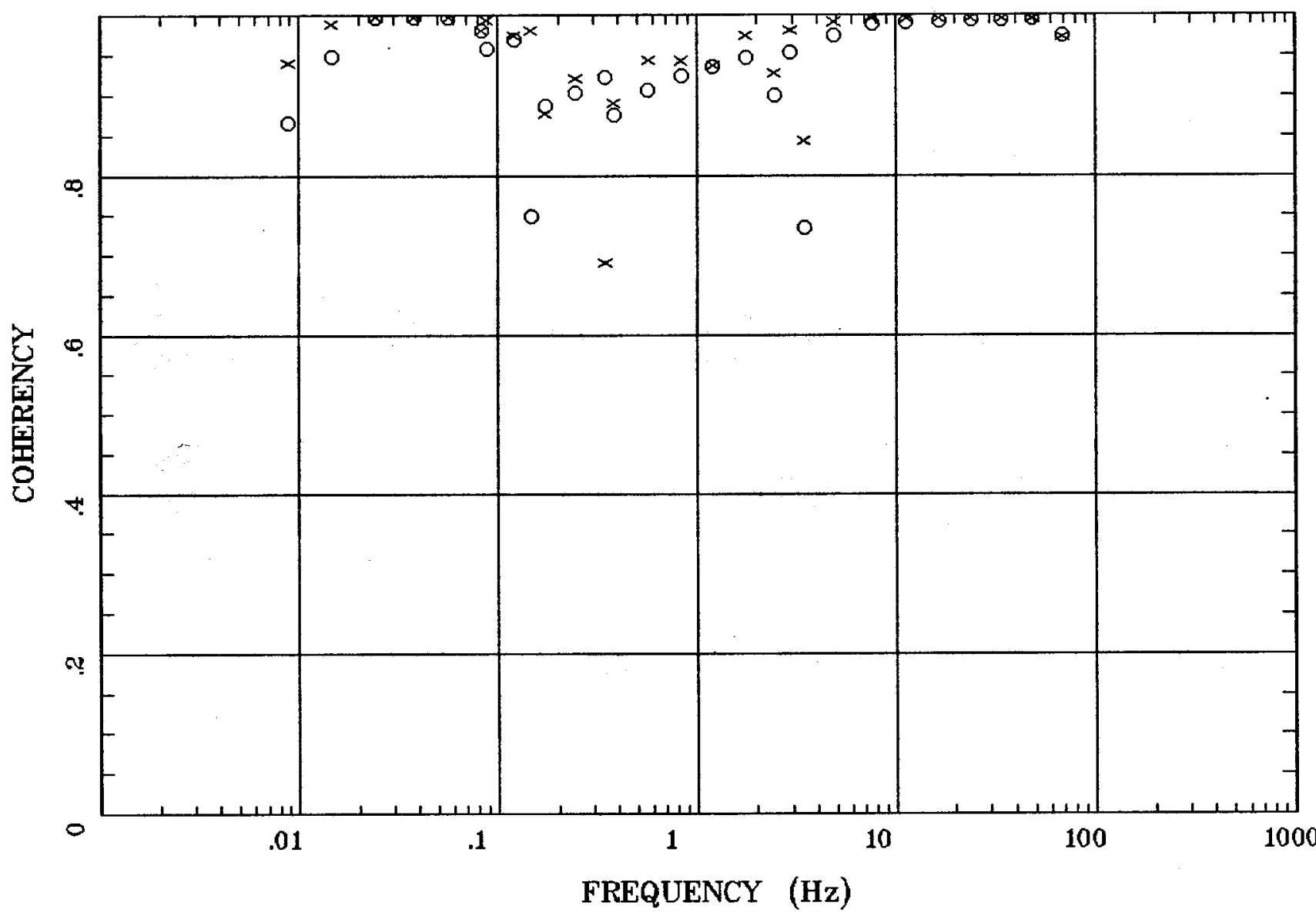

Client:

Remote: e-fld E $90 \mathrm{~m}$

Acquired: 14:2 Sep 10, 2003

Survey Co:USGS
Rotation:

Filename: ap15m.avg

Channels: Ch6 Ch7 Ch3 Ch4 Ch5 Ch6 Ch7

Plotted: 14:29 Sep 24, 2003

< EMI - ElectroMagnetic Instruments 


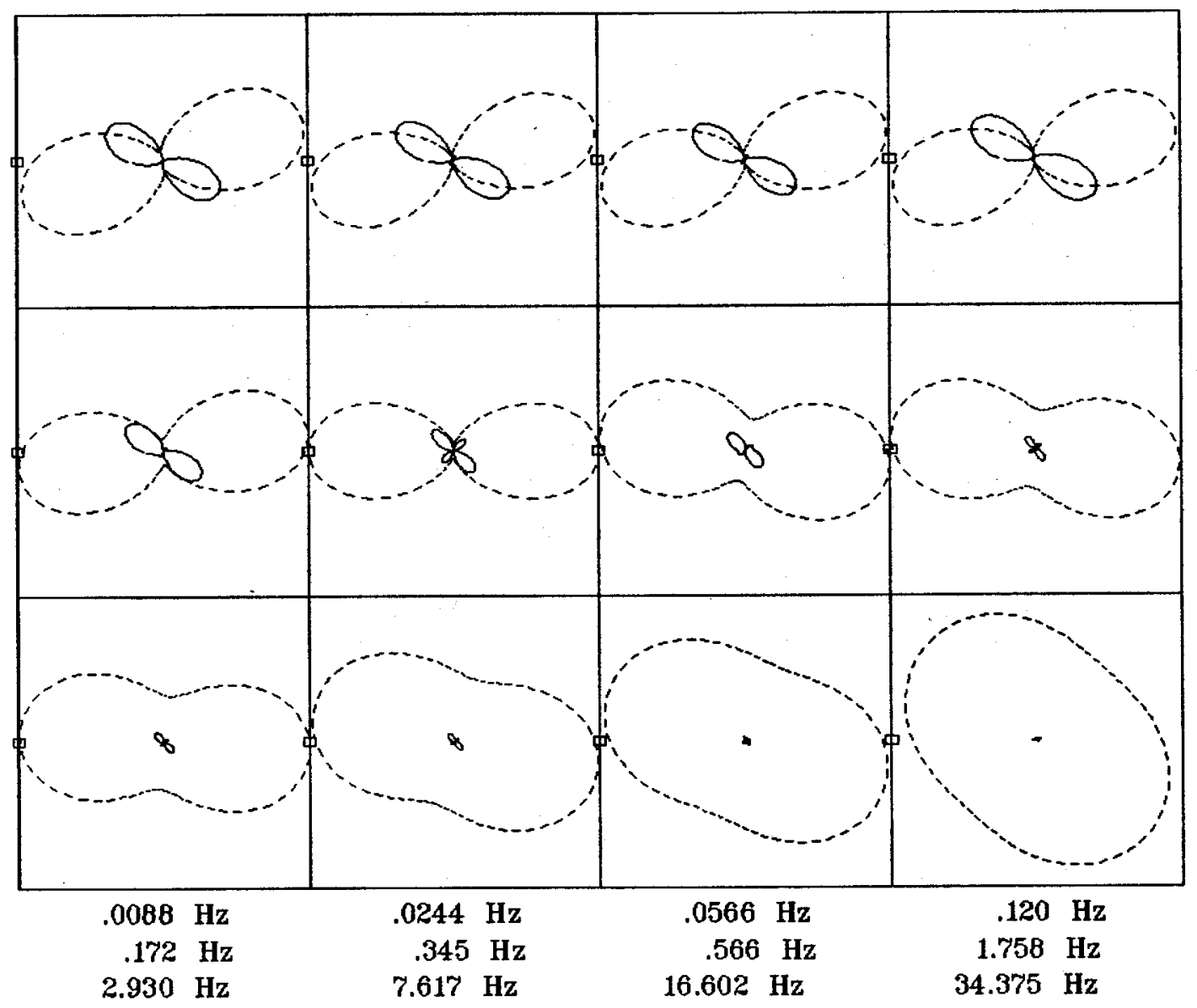

Rotation:

Client:

Remote: e-fld E $90 \mathrm{~m}$

Acquired: 14:2 Sep 10, 2003 Survey Co:USGS
Filename: ap $15 \mathrm{~m}$.avg

Channels: Ch1 Ch2 Ch3 Ch4 Ch5 Ch6 Ch7 Plotted: 08:51 Sep 24, 2003

< EMI - ElectroMagnetic Instruments > 


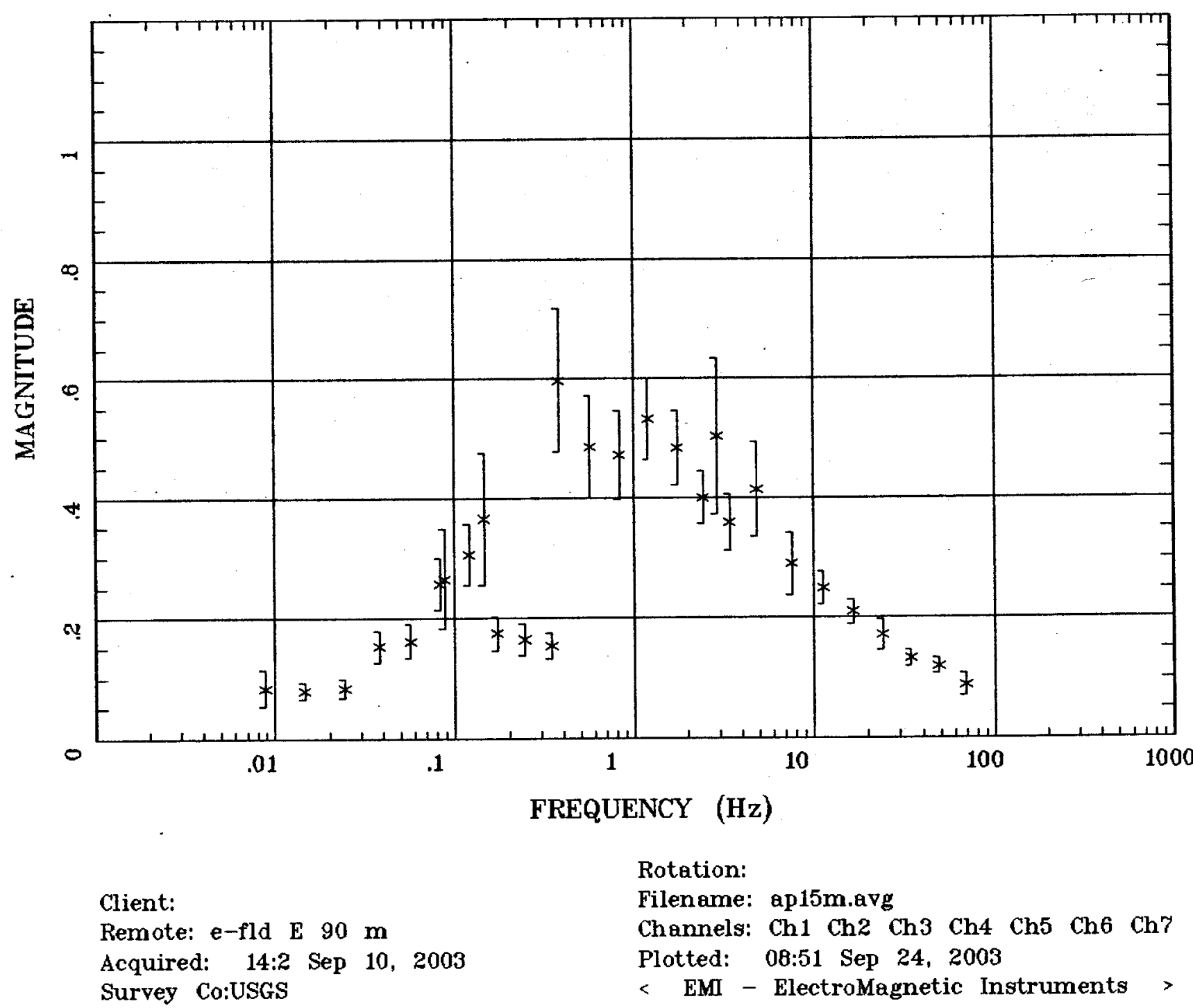




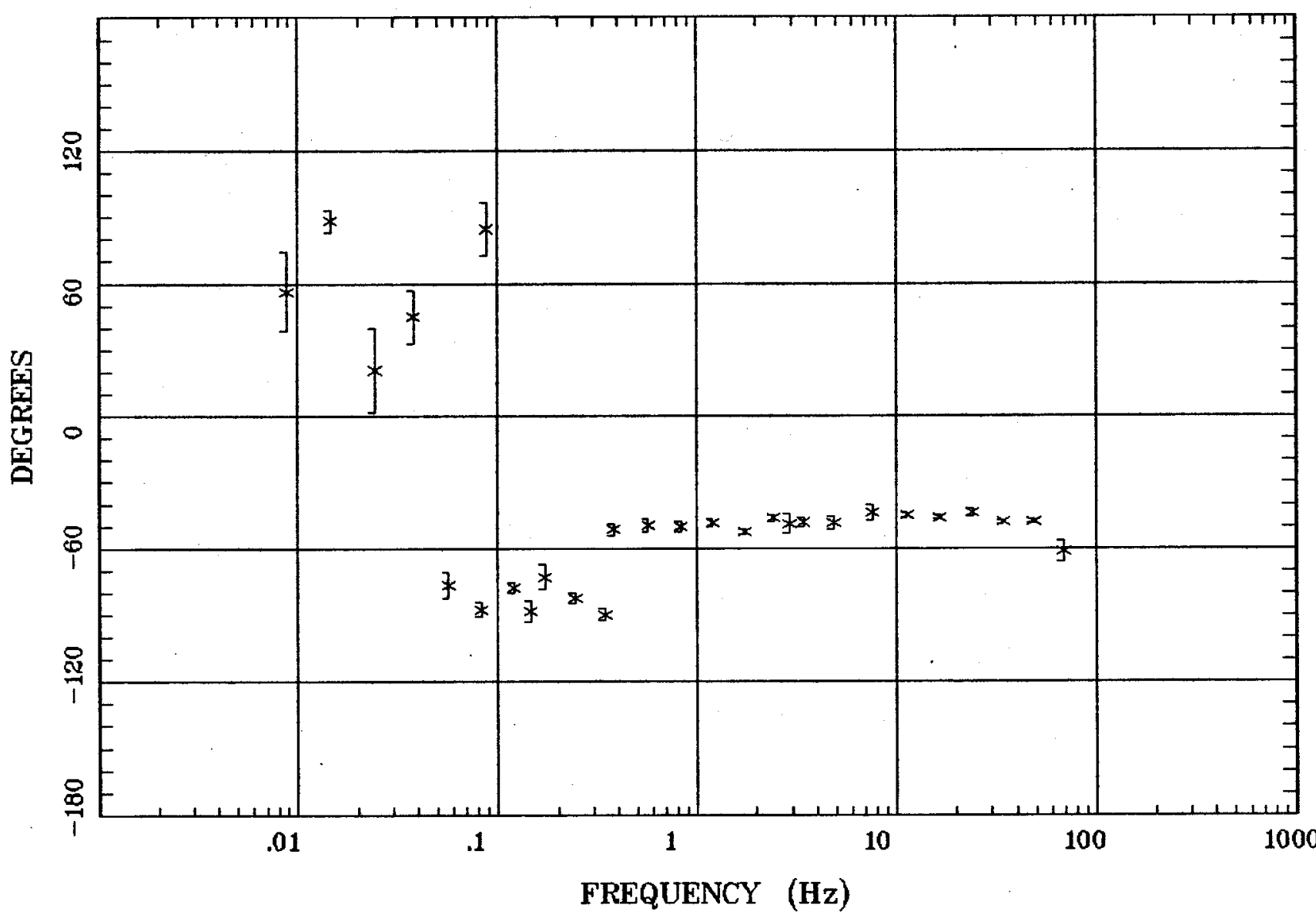

Client:

Remote: e-fld E $90 \mathrm{~m}$

Acquired: $14: 2$ Sep 10, 2003 Survey Co:USGS
Rotation:

Filename: ap $15 \mathrm{~m} . \mathrm{avg}$

Channels: Ch1 Ch2 Ch3 Ch4 Ch5 Ch6 Ch7 Plotted: 08:51 Sep 24, 2003

$<$ EMI - ElectroMagnetic Instruments 


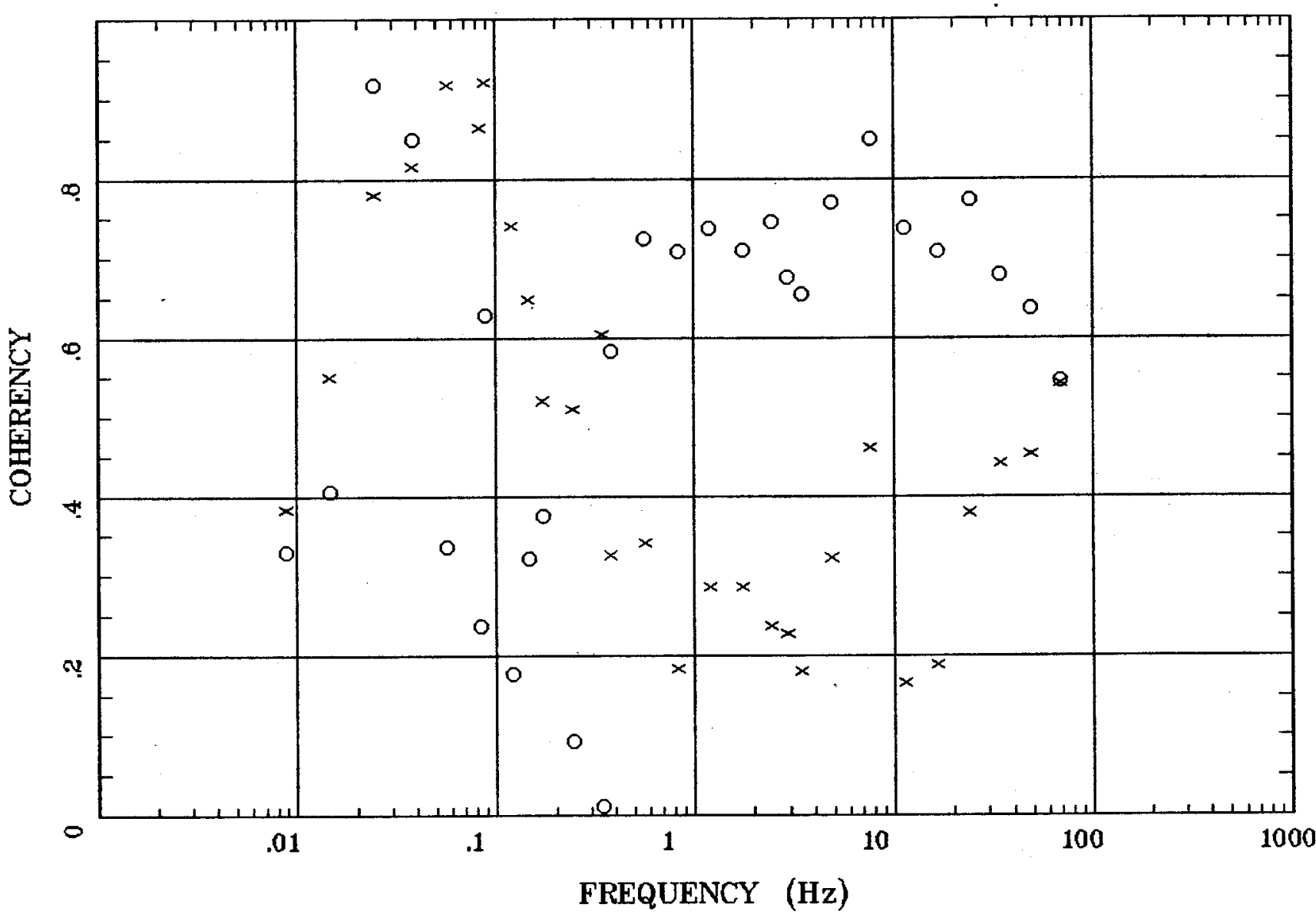

Client:

Remote: e-fld E $90 \mathrm{~m}$ Acquired: 14:2 Sep 10, 2003 Survey Co:USGS
Rotation:

Filename: ap15m.avg

Channels: Ch1 Ch2 Ch3 Ch4 Ch5 Ch6 Ch7 Plotted: 08:51 Sep 24, 2003

< EMI - ElectroMagnetic Instruments > 
Kern Mts., NV 100k Station 16

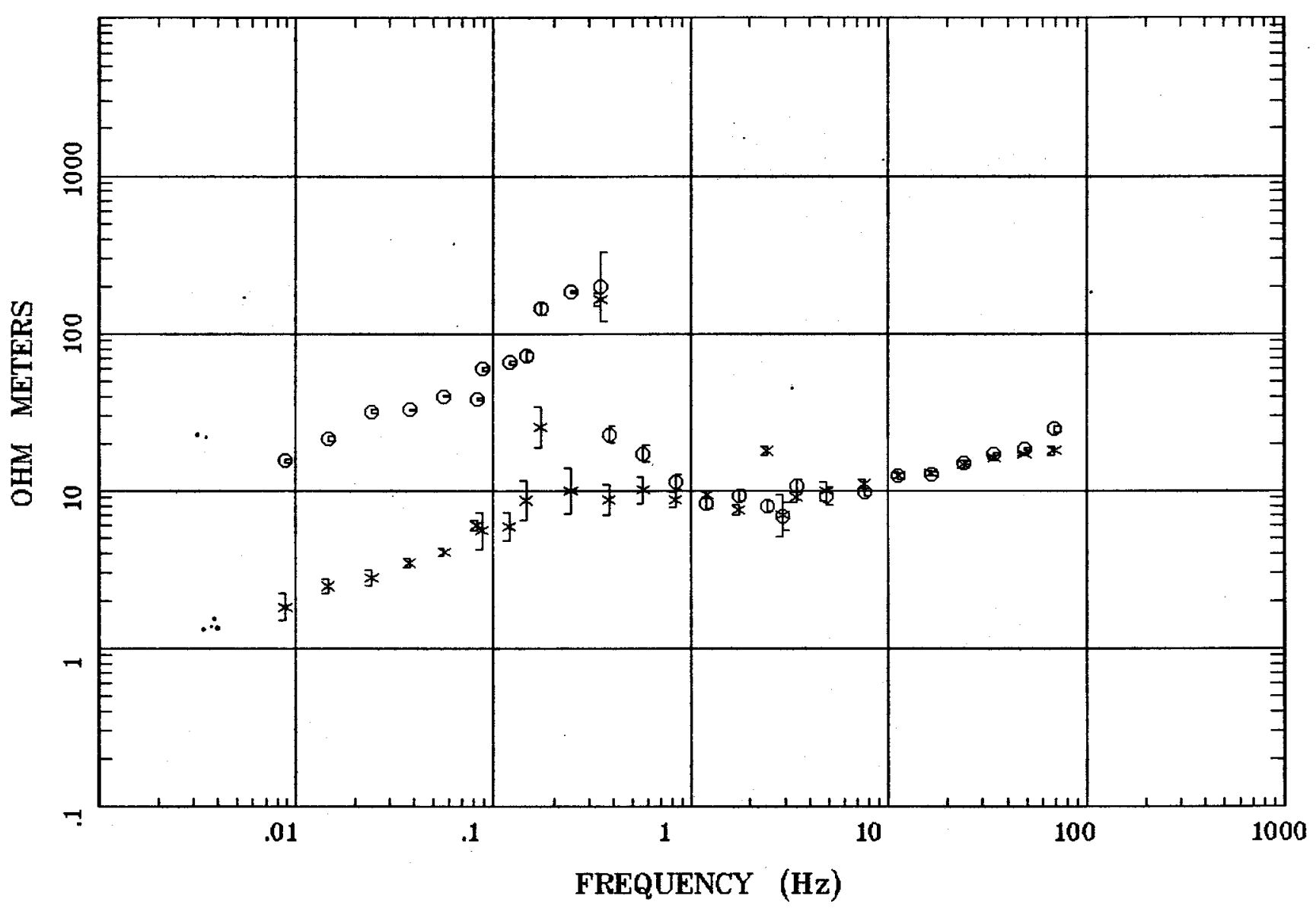

Client:

Remote: e-fld N $90 \mathrm{~m}$

Acquired: 10:4 Sep 11, 2003

Survey Co:USGS

Rotation:

Filename: ap16m.avg

Channels: Ch1 Ch2 Ch3 Ch4 Ch5 Ch6 Ch7

Plotted: 14:16 Sep 24, 2003

< EMI - ElectroMagnetic Instruments > 


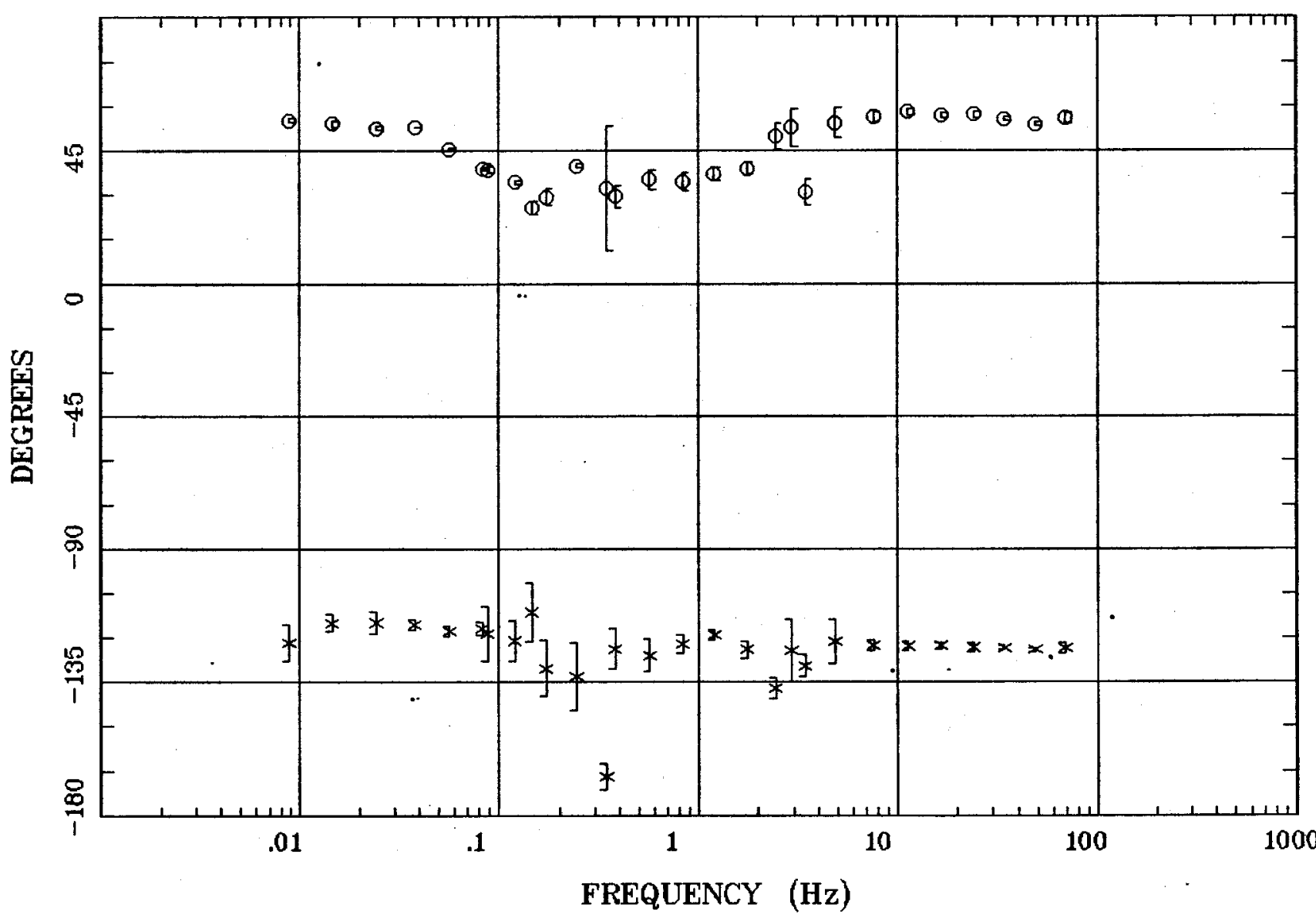

Client:

Remote: e-fld N $90 \mathrm{~m}$

Acquired: 10:4 Sep 11, 2003 Survey Co:USGS
Rotation:

Filename: ap16m.avg

Channels: Ch1 Ch2 Ch3 Ch4 Ch5 Ch6 Chry Platted: 14:16 Sep 24, 2003

< EMI - ElectroMagnetic Instruments 


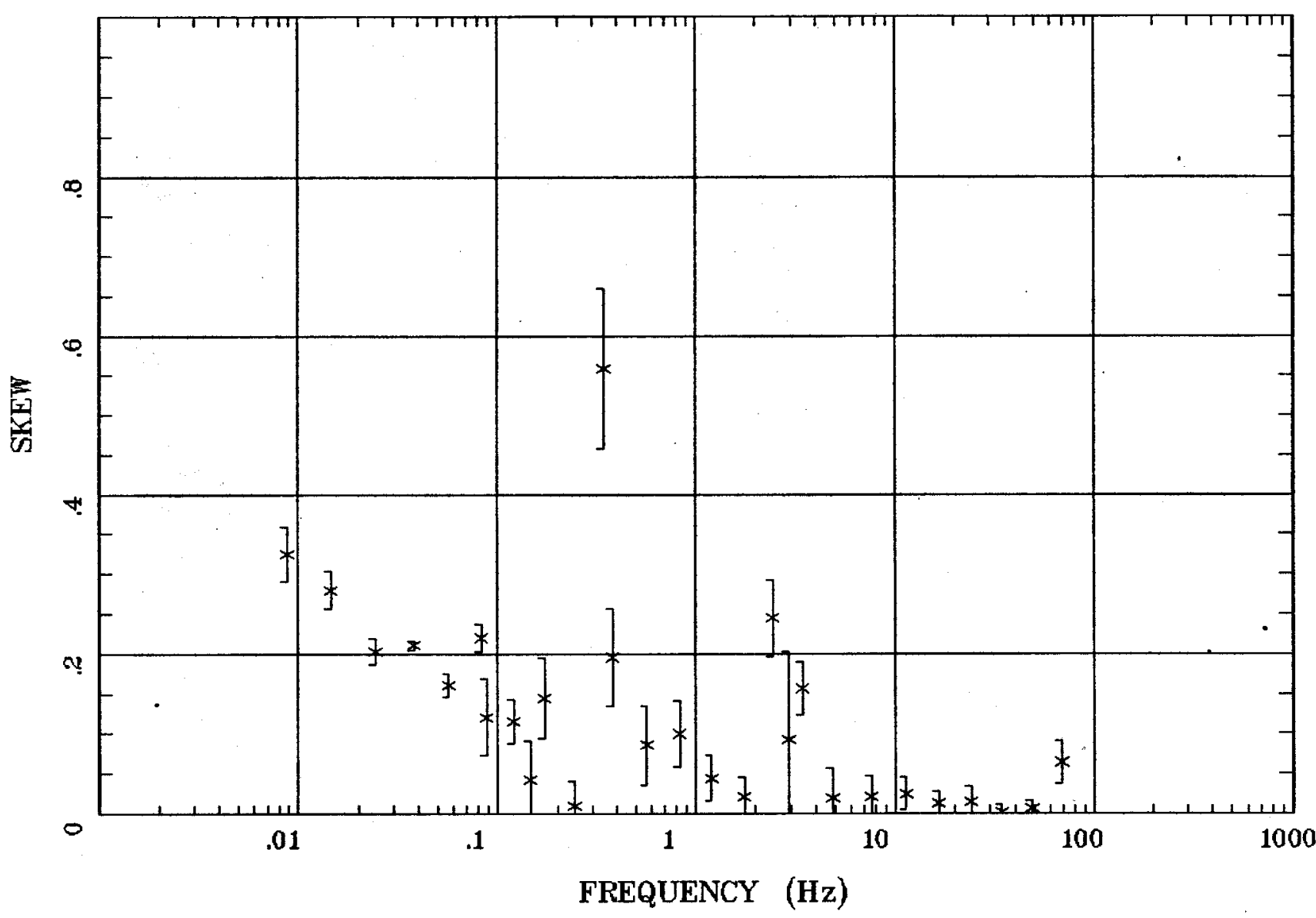

Client:

Remote: e-fld N $90 \mathrm{~m}$ Acquired: $10: 4$ Sep 11, 2003 Survey Co:USGS
Rotation:

Filename: ap16m.avg

Channels: Ch1 Ch2 Ch3 Ch4 Ch5 Ch6 Ch7 Plotted: $14: 16$ Sep 24, 2003

$<$ EMI - ElectroMagnetic Instruments 
E MULT Coh.

Kern Mts., NV 100k Station 16

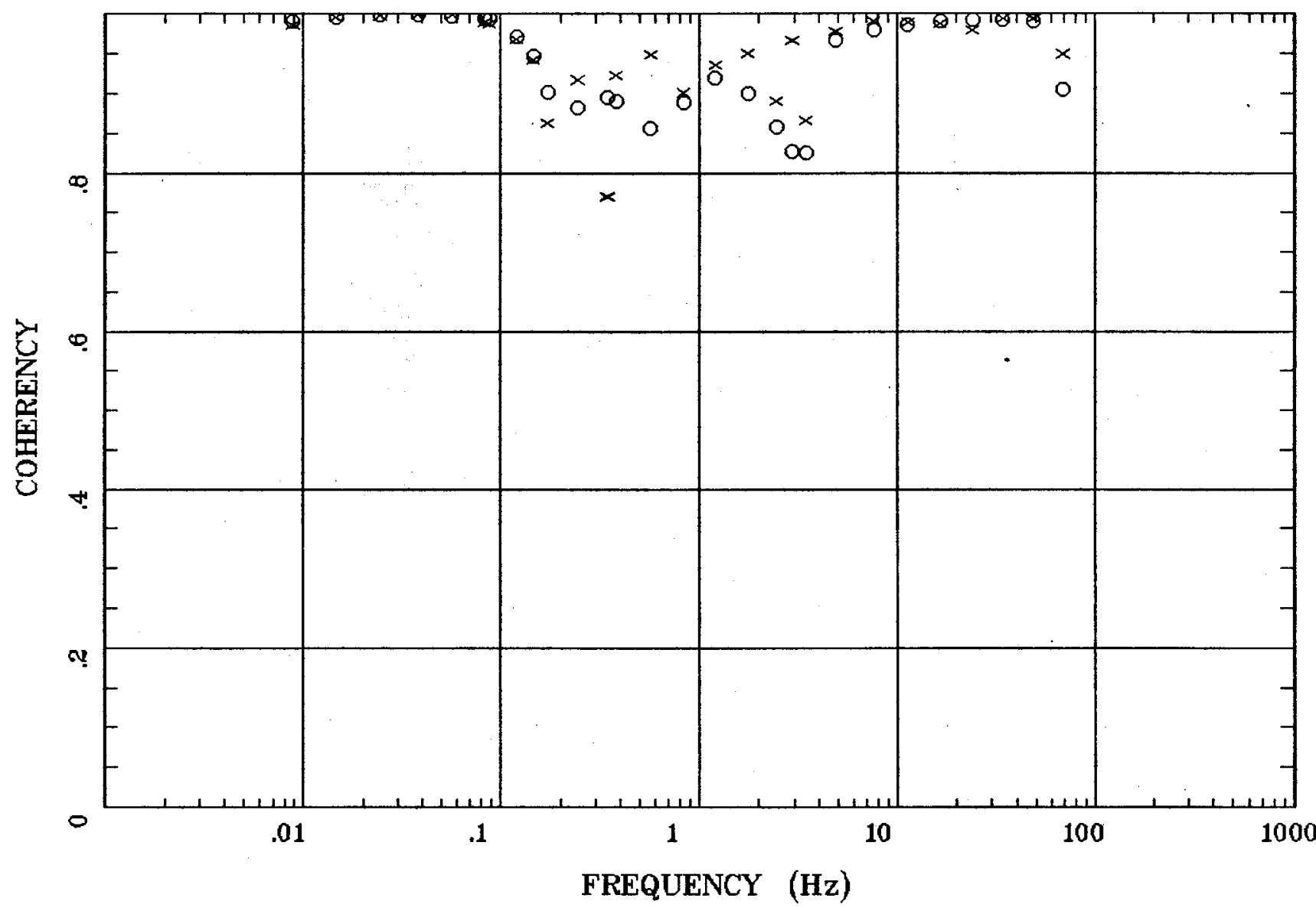

Client:

Remote: e-fld N $90 \mathrm{~m}$ Acquired: $10: 4$ Sep 11, 2003 Survey Co:USGS
Rotation:

Filename: ap16m.avg

Channels: Ch1 Ch2 Ch3 Ch4 Ch5 Ch6 Chr

Plotted: 14:16 Sep 24, 2003

$<$ EMI - ElectroMagnetic Instruments 


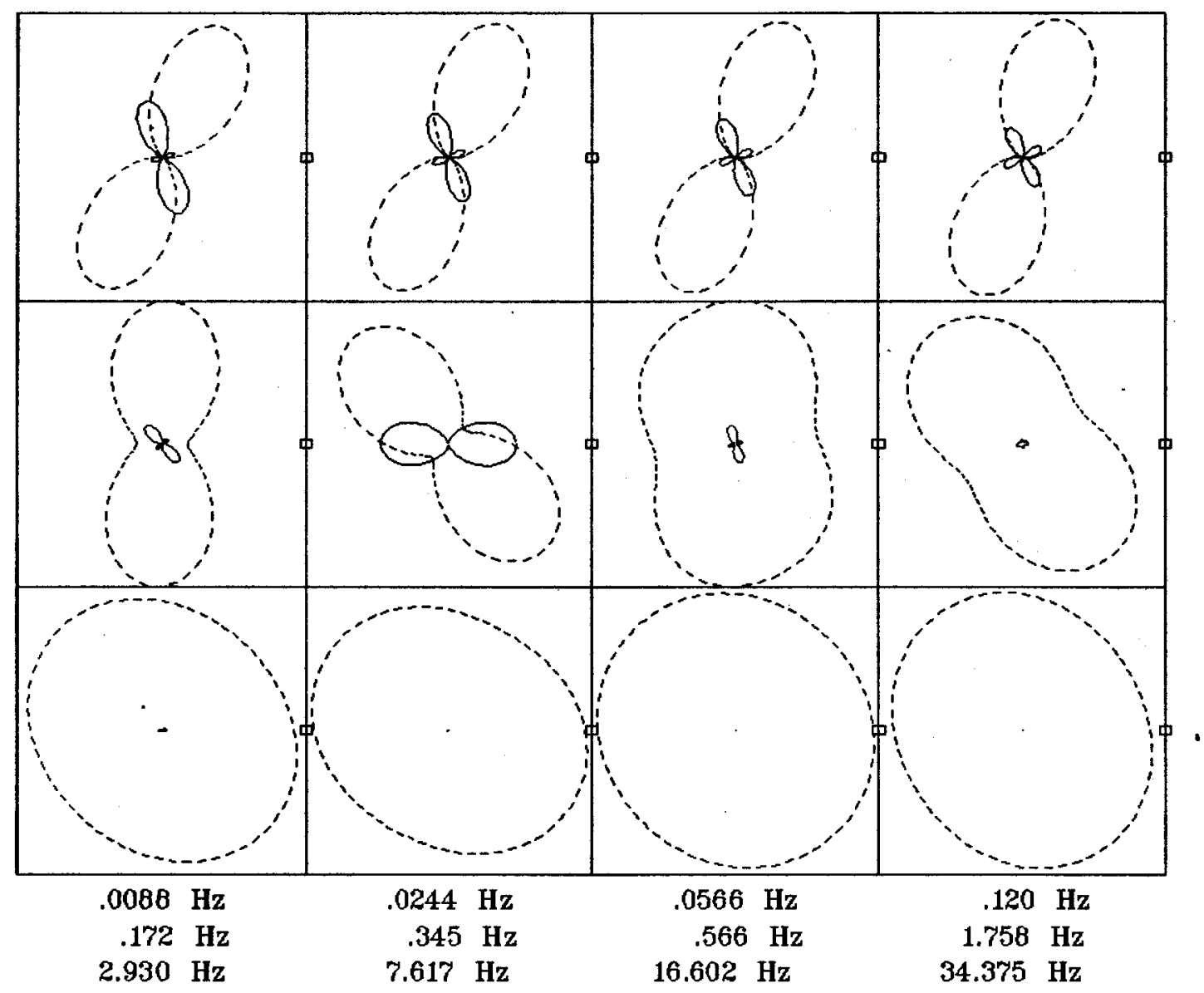

Rotation:

Client:

Remote: e-fld N $90 \mathrm{~m}$

Acquired: 10:4 Sep 11, 2003 Survey Co:USGS
Filename: ap16m.avg

Channels: Ch1 Ch2 Ch3 Ch4 Ch5 Ch6 Ch7 Plotted: 14:16 Sep 24, 2003

$<$ EMI - ElectroMagnetic Instruments 


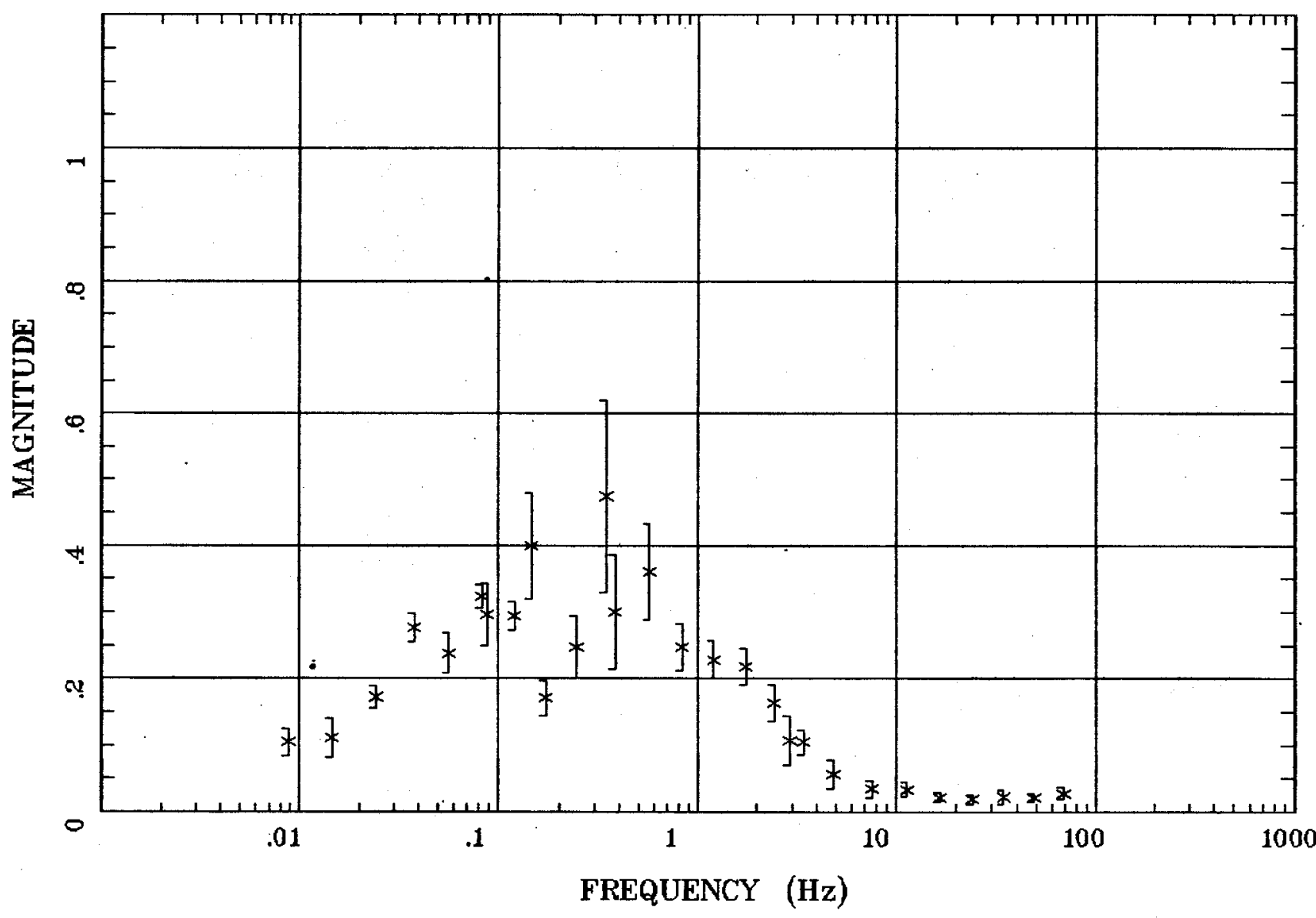

Client:

Remote: e-fld N $90 \mathrm{~m}$

Acquired: 10:4 Sep 11, 2003 Survey Co:USGS

\section{Rotation:}

Filename: ap16m.avg

Channels: Ch1 Ch2 Ch3 Ch4 Ch5 Ch6 Ch7 Plotted: 14:16 Sep 24, 2003

$<$ EMI - ElectroMagnetic Instruments 


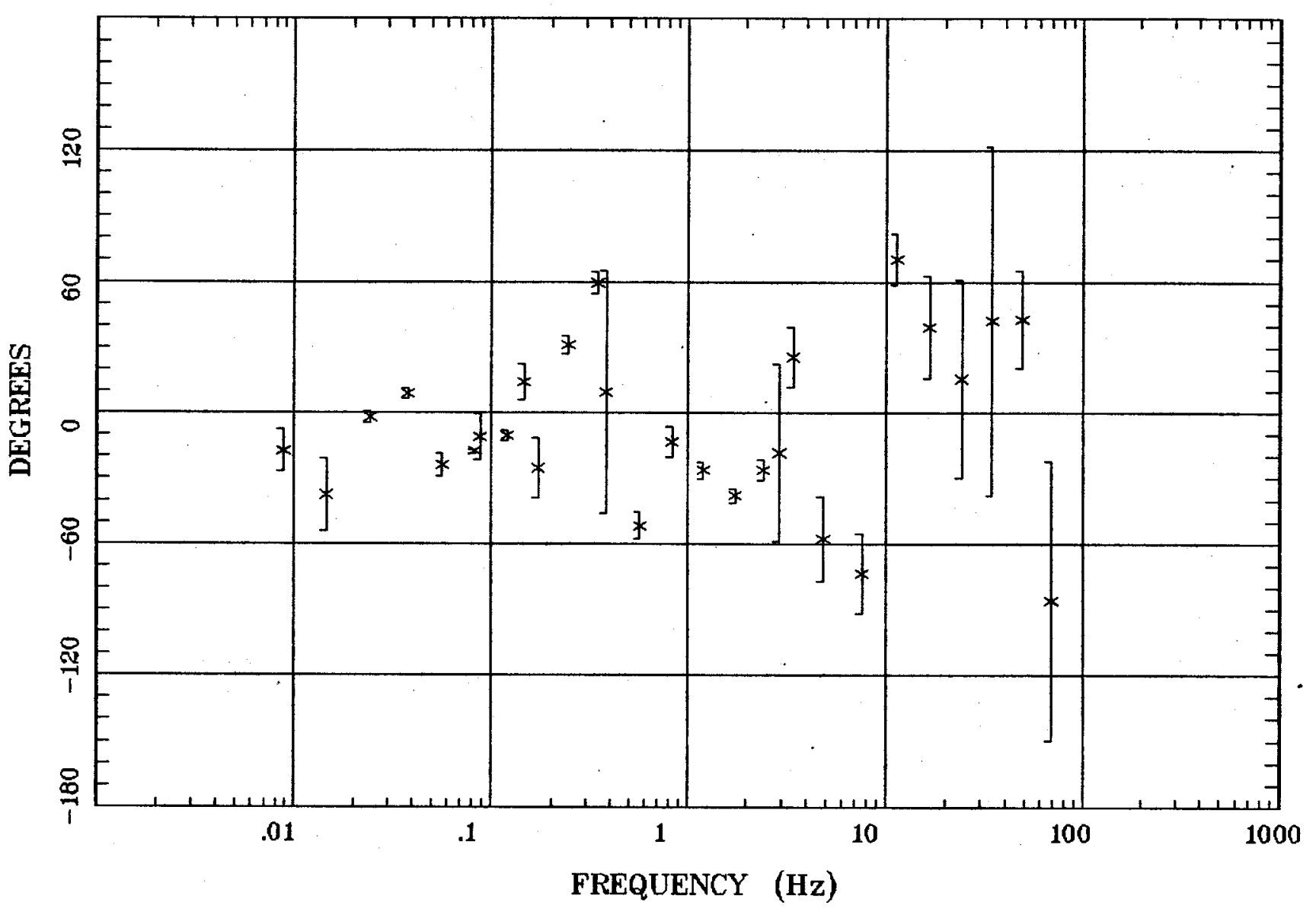

Client:

Remote: e-fld N $90 \mathrm{~m}$

Acquired: 10:4 Sep 11, 2003

Survey Co:USGS
Rotation:

Filename: ap16m.avg

Channels: Ch1 Ch2 Ch3 Ch4 Ch5 Ch6 Ch7

Plotted: 14:16 Sep 24, 2003

$<$ EMI - ElectroMagnetic Instruments 


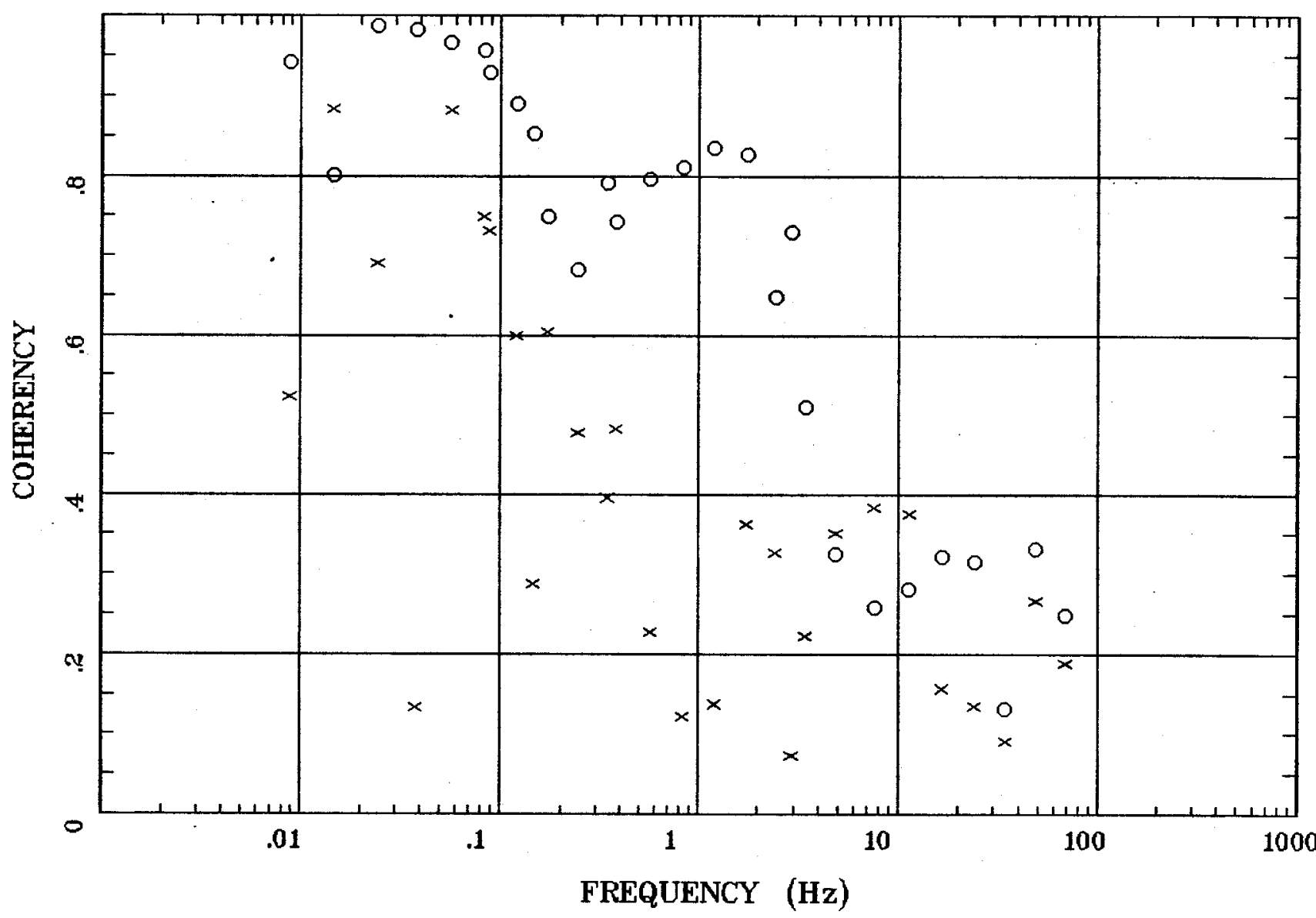

Client:

Remote: e-fld N $90 \mathrm{~m}$

Acquired: 10:4 Sep 11, 2003 Survey Co:USGS
Rotation:

Filename: ap $16 \mathrm{~m} \cdot \mathrm{avg}$

Channels: Ch1 Ch2 Ch3 Ch4 Ch5 Ch6 Ch7

Plotted: 14:16 Sep 24, 2003

$<$ EMI - ElectroMagnetic Instruments 
Kern Mts., NV 100k Station 17

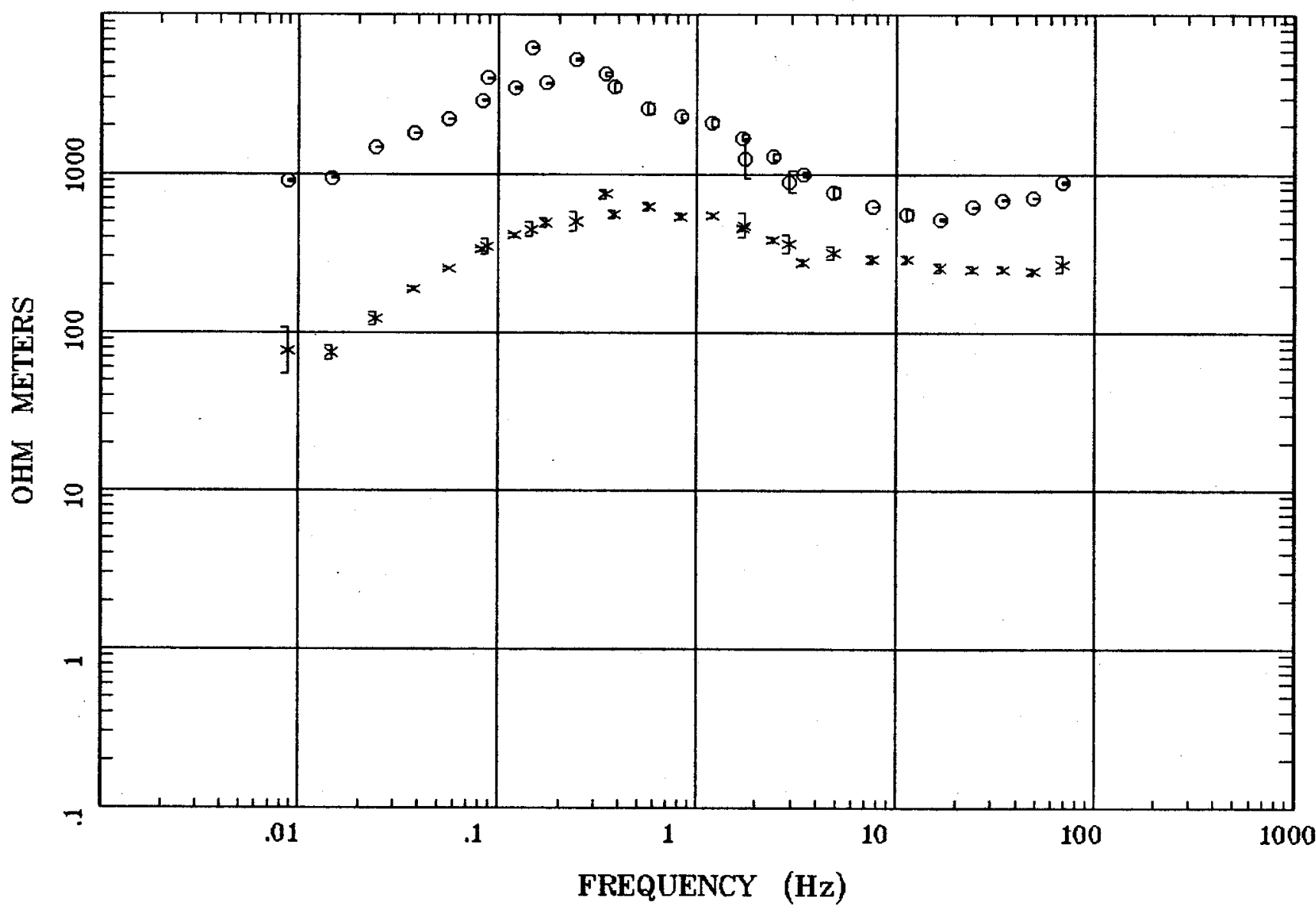

Client:

Remote: e-fld Fest $90 \mathrm{~m}$ Acquired: 14:1 Sep 11, 2003 Survey Co:USGS
Rotation:

Filename: ap17mall.avg

Channels: Ch1 Ch2 Ch3 Ch4 Ch5 Ch6 Ch7 Plotted: 11:19 Sep 24, 2003

< EMI - ElectroMagnetic Instruments > 
Kern Mts., NV 100k Station 17

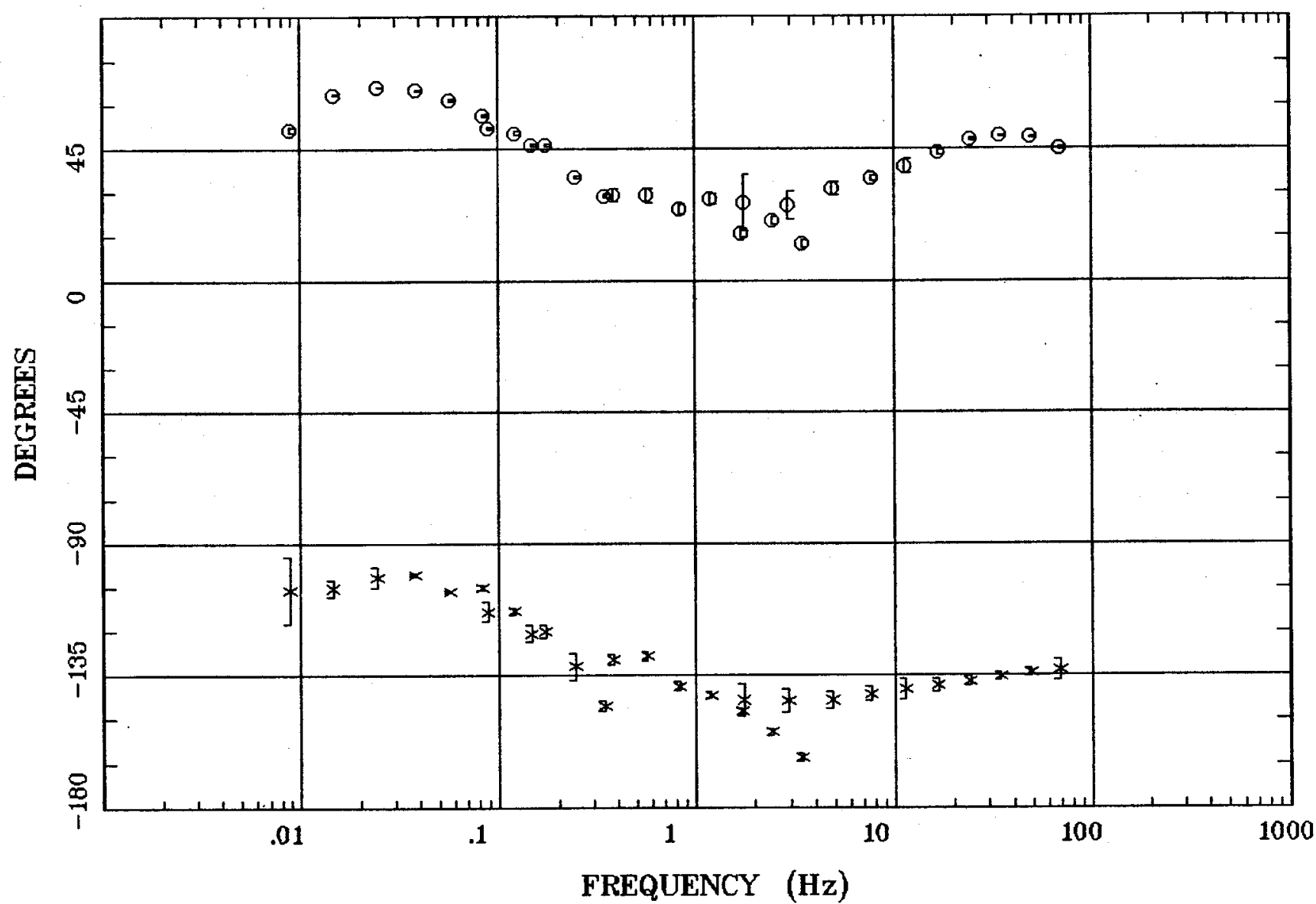

Client:

Remote: e-fld West $90 \mathrm{~m}$

Acquired: 14:1 Sep 11, 2003 Survey Co:USGS
Rotation:

Filename: ap17mall.avg

Channels: Ch1 Ch2 Ch3 Ch4 Ch5 Ch6 Ch7 Plotted: 11:19 Sep 24, 2003

< EMI - ElectroMagnetic Instruments 
Kern Mts., NV 100k Station 17

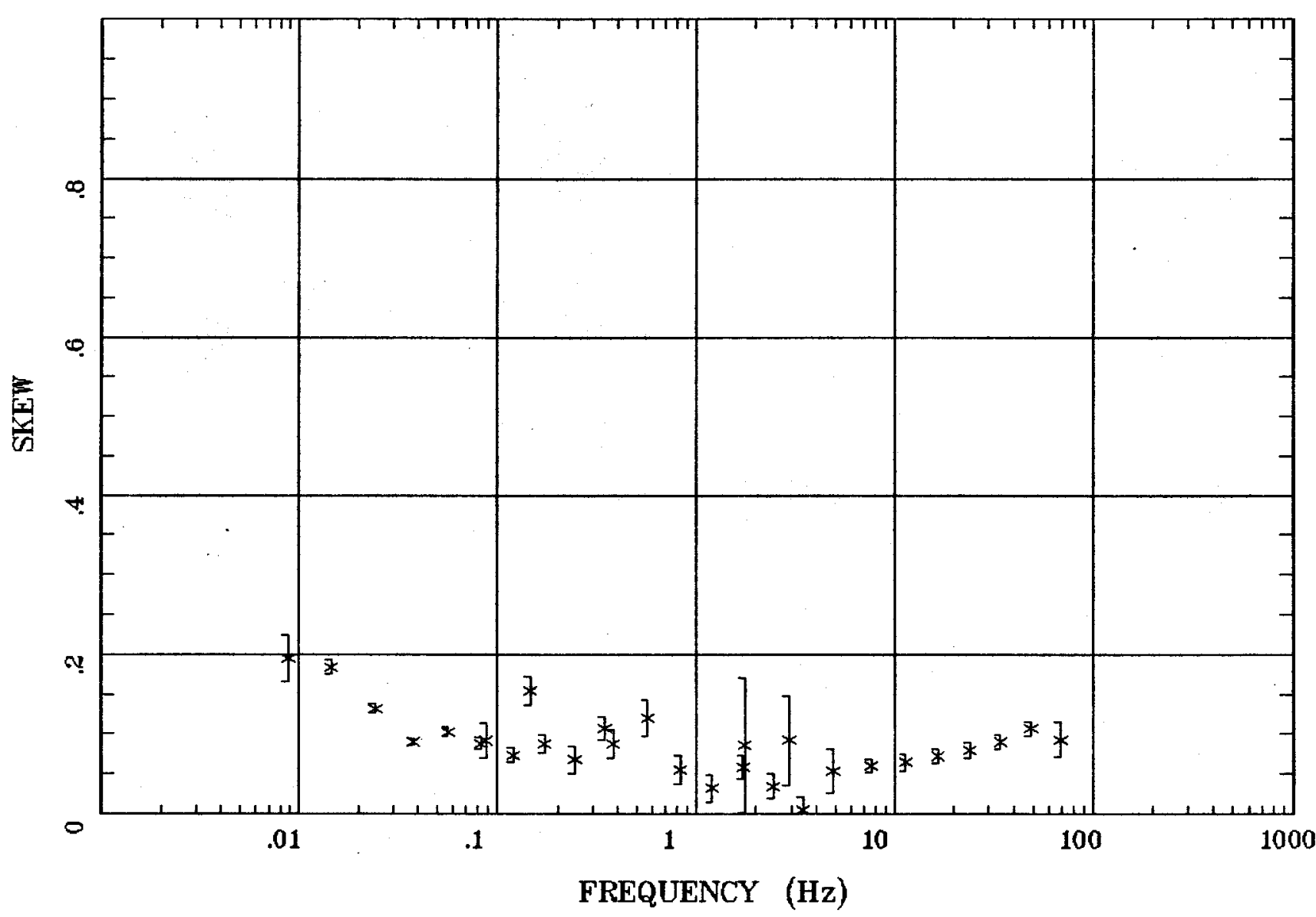

Client:

Remote: e-fld West $90 \mathrm{~m}$

Acquired: 14:1 Sep 11, 2003

Survey Co:USGS
Rotation:

Filename: ap17mall.avg

Channels: Ch1 Ch2 Ch3 Ch4 Ch5 Ch6 Ch7

Plotted: 11:19 Sep 24, 2003

$<$ EMI - ElectroMagnetic Instruments 


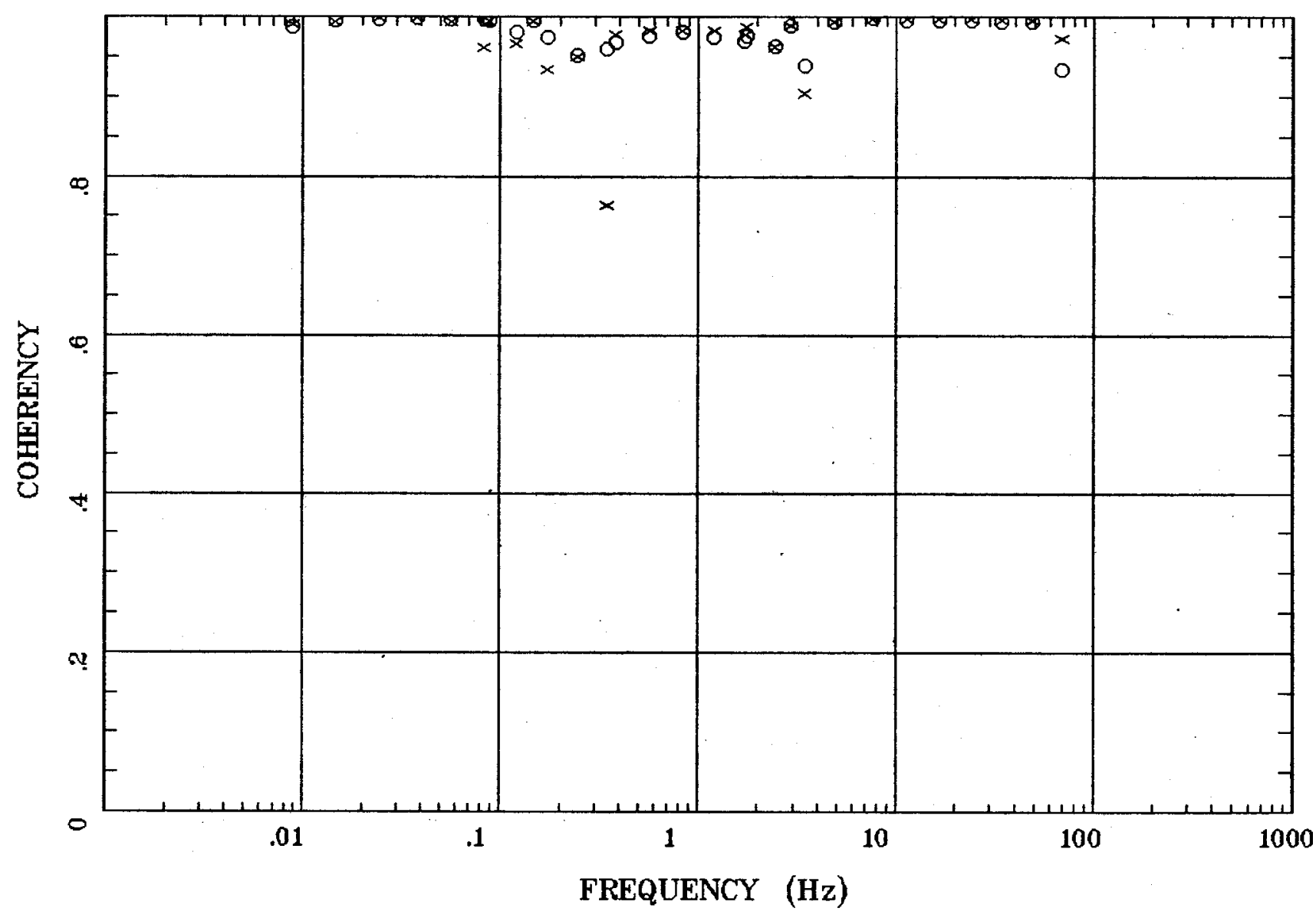

Client:

Remote: e-fld West $90 \mathrm{~m}$

Acquired: $14: 1$ Sep 11, 2003 Survey Co:USGS
Rotation:

Filename: ap17mall.avg

Channels: Ch1 Ch2 Ch3 Ch4 Ch5 Ch6 Ch7 Plotted: 11:19 Sep 24, 2003

< EMI - ElectroMagnetic Instruments 


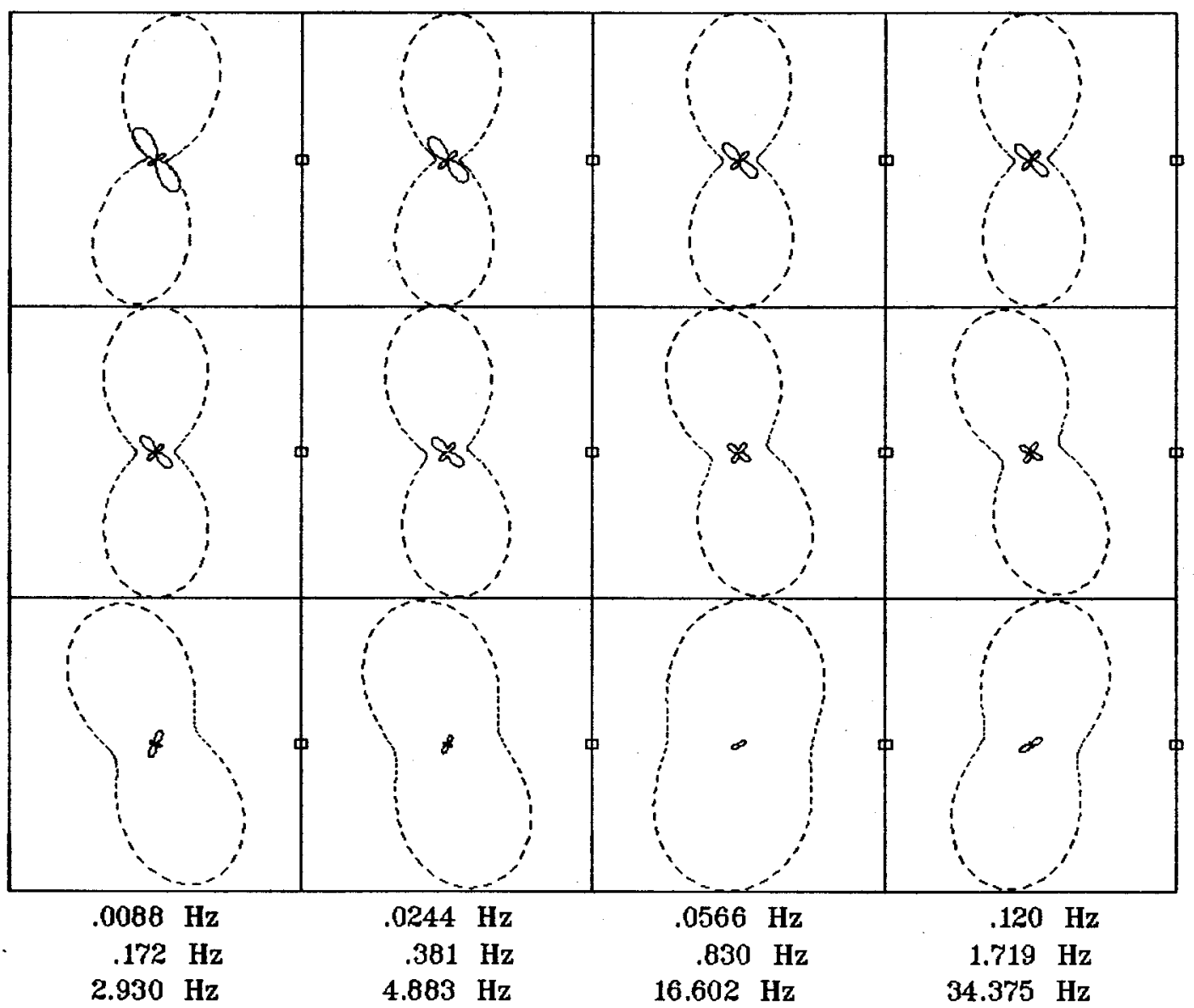

Client:

Remote: e-fld Fest $90 \mathrm{~m}$

Acquired: 14:1 Sep 11, 2003

Survey Co:USGS
Rotation:

Filename: ap17mall.avg

Channels: Ch1 Ch2 Ch3 Ch4 Ch5 Ch6 Ch7

Plotted: 11:19 Sep 24, 2003

$<$ EMI - ElectroMagnetic Instruments > 


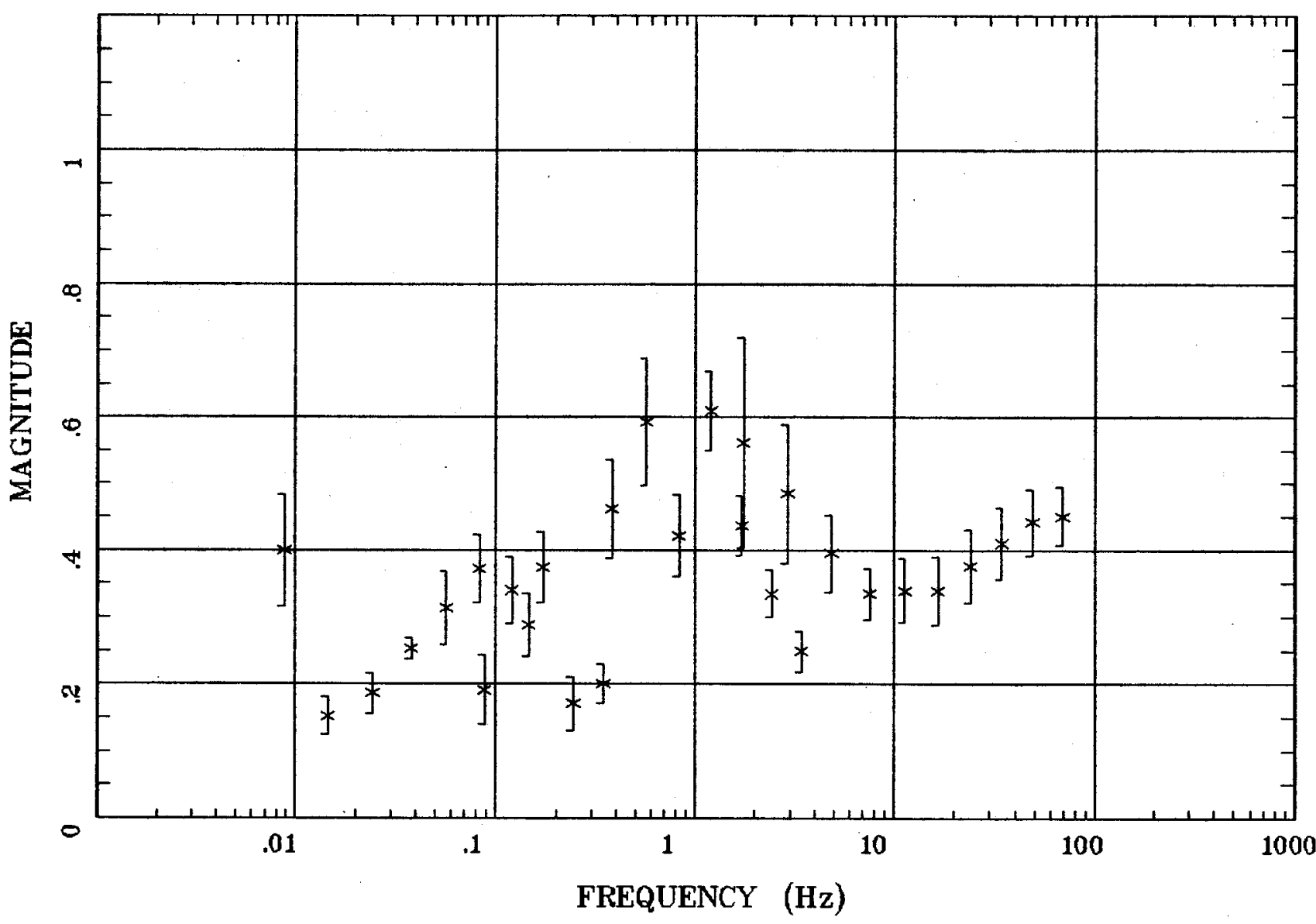

Client:

Remote: e-fld Fest $90 \mathrm{~m}$

Acquired: 14:1 Sep 11, 2003 Survey Co:USGS
Rotation:

Filename: ap17mall.avg

Channels: Ch1 Ch2 Ch3 Ch4 Ch5 Ch6 Ch7 Plotted: 11:19 Sep 24, 2003

$<$ EMI - ElectroMagnetic Instruments > 


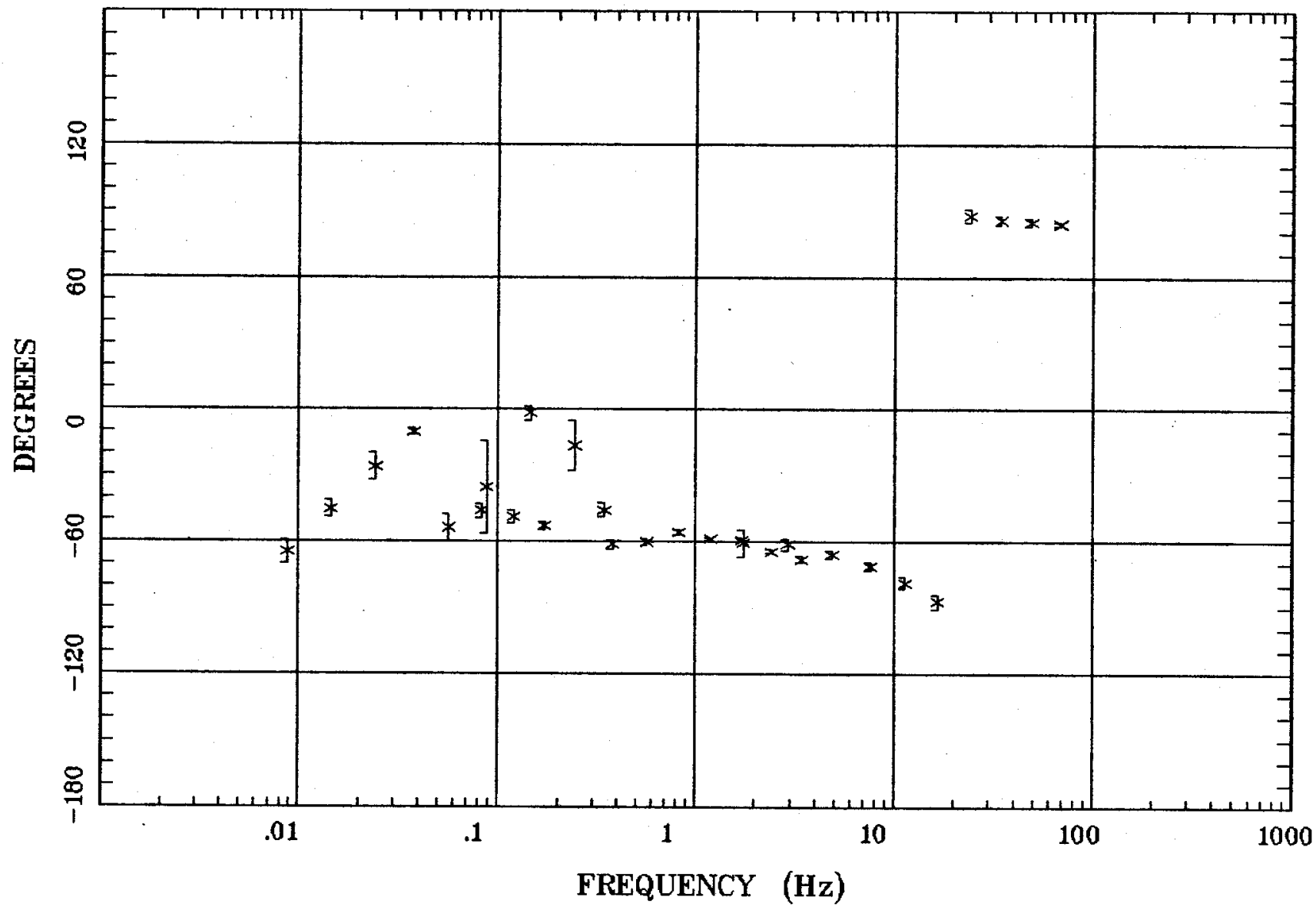

Client:

Remote: e-fld West $90 \mathrm{~m}$

Acquired: 14:1 Sep 11, 2003

Survey Co:USGS
Rotation:

Filename: ap17mall.avg

Channels: Ch1 Ch2 Ch3 Ch4 Ch5 Ch6 Ch7

Plotted: 11:19 Sep 24, 2003

$<$ EMI - ElectroMagnetic Instruments 
HzHx.x Coh HzHy.o

Kern Mts., NV 100k Station 17

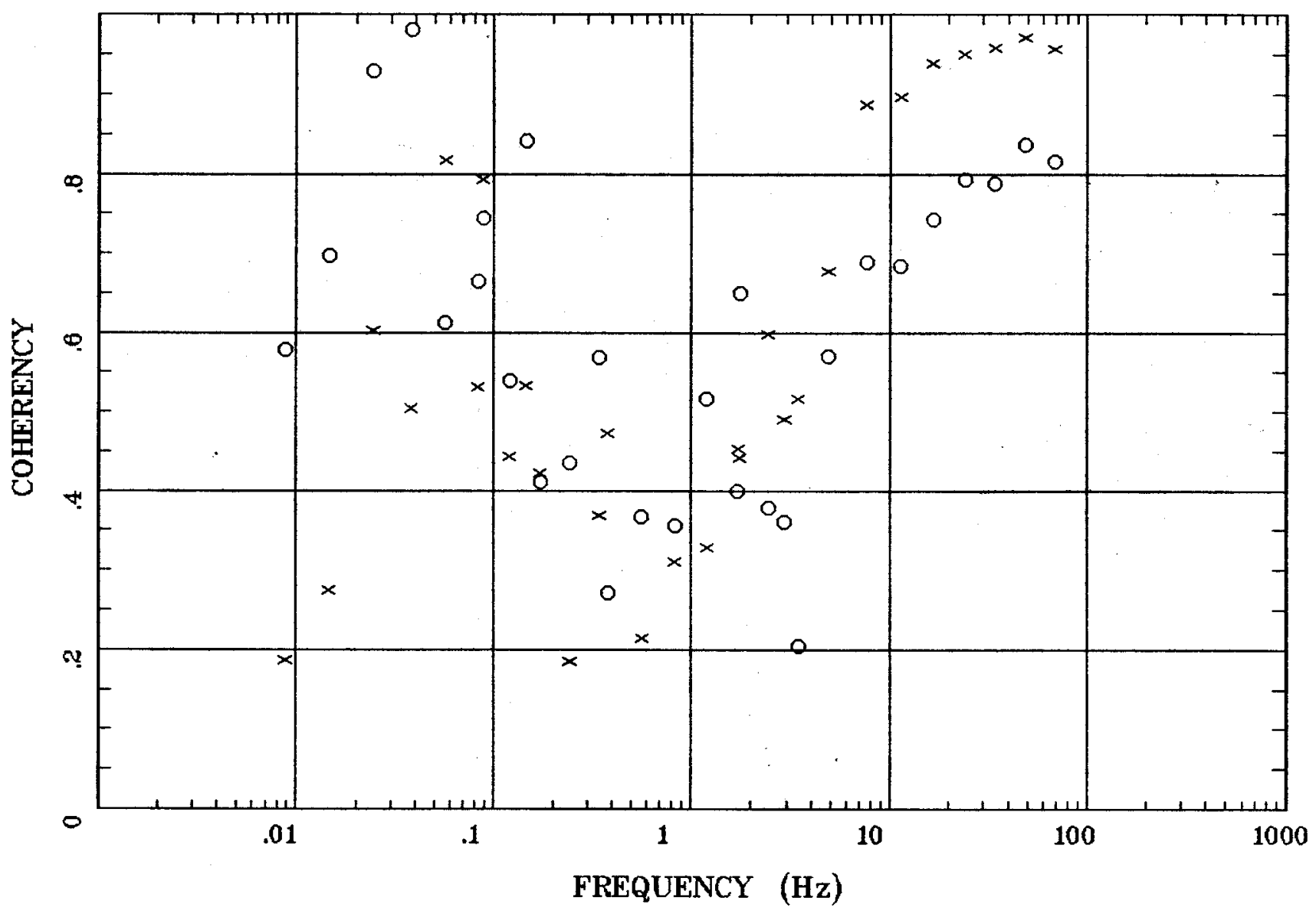

Client:

Remote: e-fld Fest $90 \mathrm{~m}$ Acquired: 14:1 Sep 11, 2003 Survey Co:USGS
Rotation:

Filename: ap17mall.avg

Channels: Ch1 Ch2 Ch3 Ch4 Ch5 Ch6 Ch7

Plotted: 11:19 Sep 24, 2003

< EMI - ElectroMagnetic Instruments 


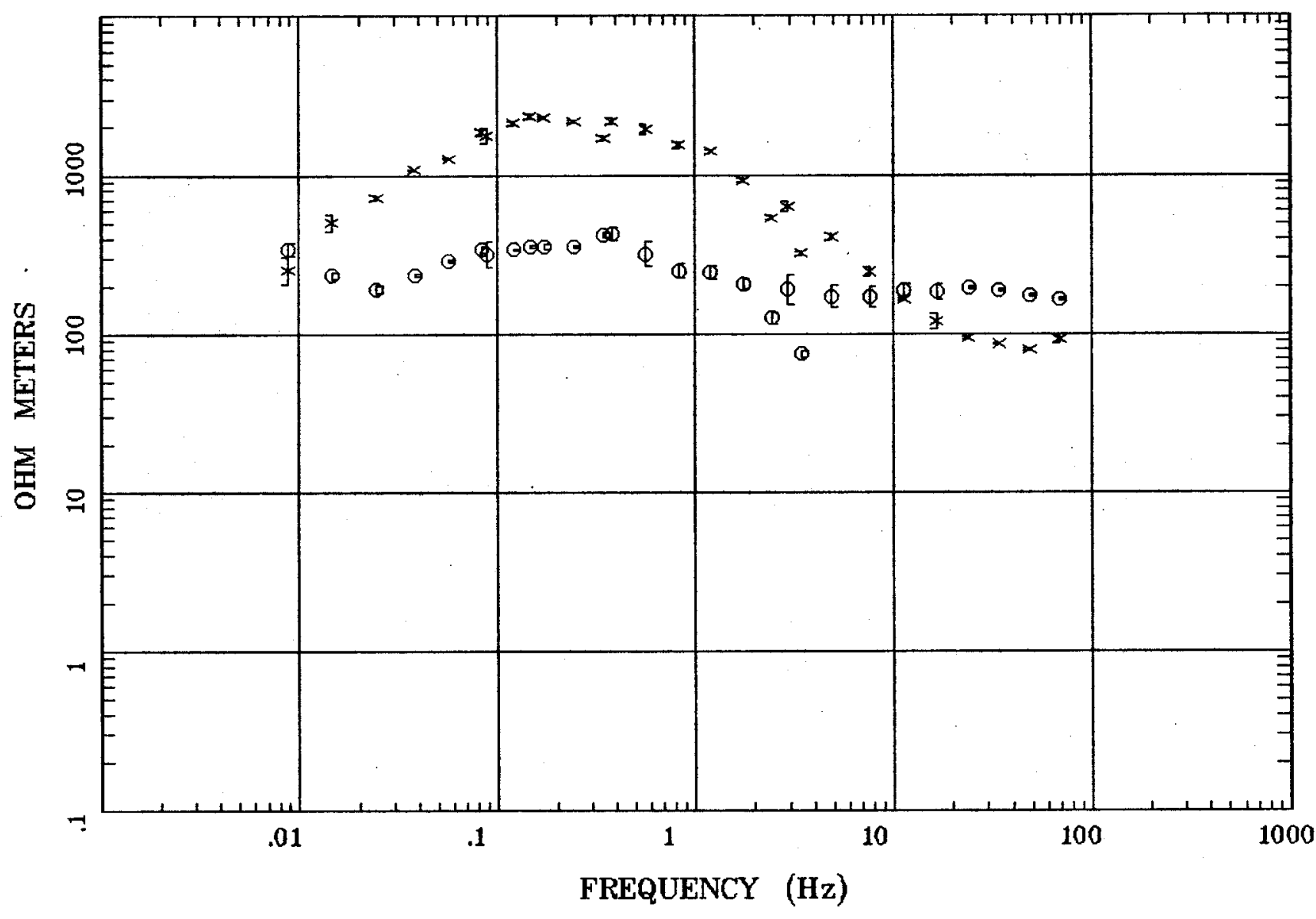

Client:

Remote: e-fld North $90 \mathrm{~m}$ Acquired: 10:4 Sep 16, 2003 Survey Co:USGS
Rotation:

Filename: ap18m.avg

Channels: Ch1 Ch2 Ch3 Ch4 Ch5 Ch6 Ch7 Plotted: 11:20 Sep 24, 2003

< EMI - ElectroMagnetic Instruments 
Kern Mts., NV 100k Station 18

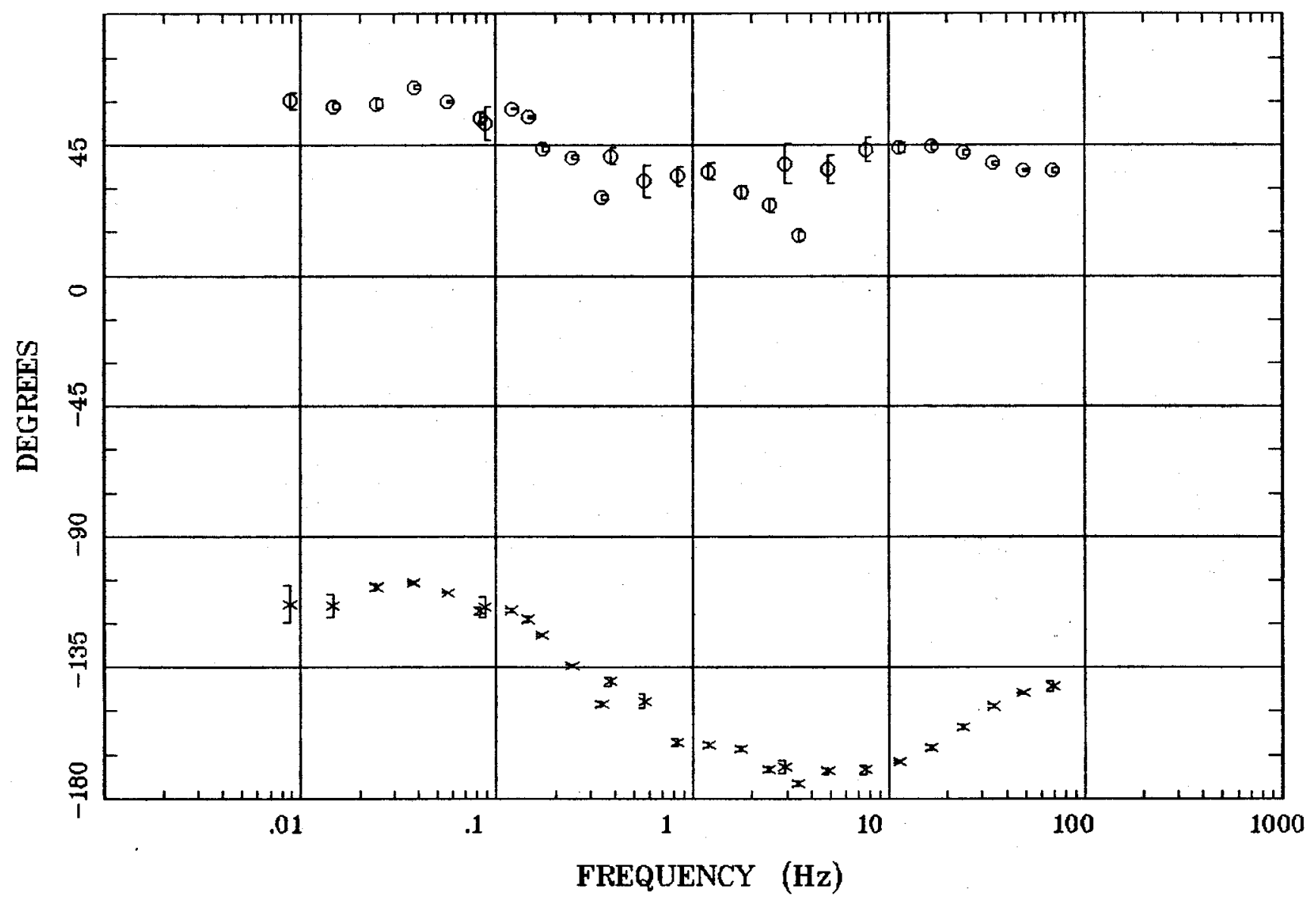

Client:

Remote: e-fld North $90 \mathrm{~m}$ Acquired: 10:4 Sep 16, 2003 Survey Co:USGS
Rotation:

Filename: ap $18 \mathrm{~m} . \mathrm{avg}$

Channels: Ch1 Ch2 Ch3 Ch4 Ch5 Ch6 Ch7

Plotted: 11:20 Sep 24, 2003

< EMI - ElectroMagnetic Instruments 
Kern Mts., NV $100 \mathrm{k}$

Station 18

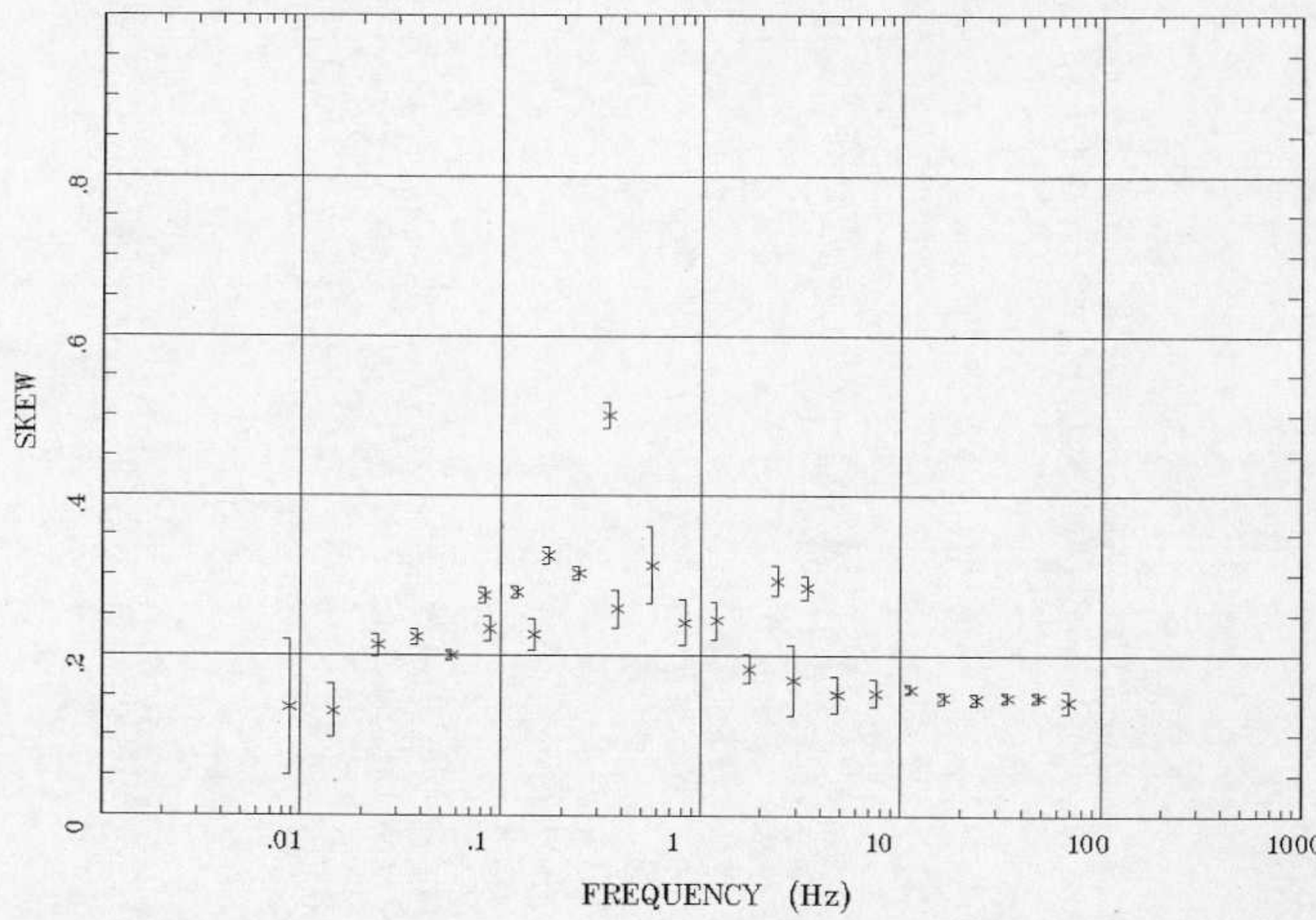

Client:

Remote: e-fld North $90 \mathrm{~m}$ Acquired: 10:4 Sep 16, 2003

Survey Co:USGS
Rotation:

Filename: ap18m.avg

Channels: Ch1 Ch2 Ch3 Ch4 Ch5 Ch6 Ch7 Plotted: 11:20 Sep 24, 2003

$<$ EMI - ElectroMagnetic Instruments 
E MULT Coh.

Kern Mts., NV 100k Station 18

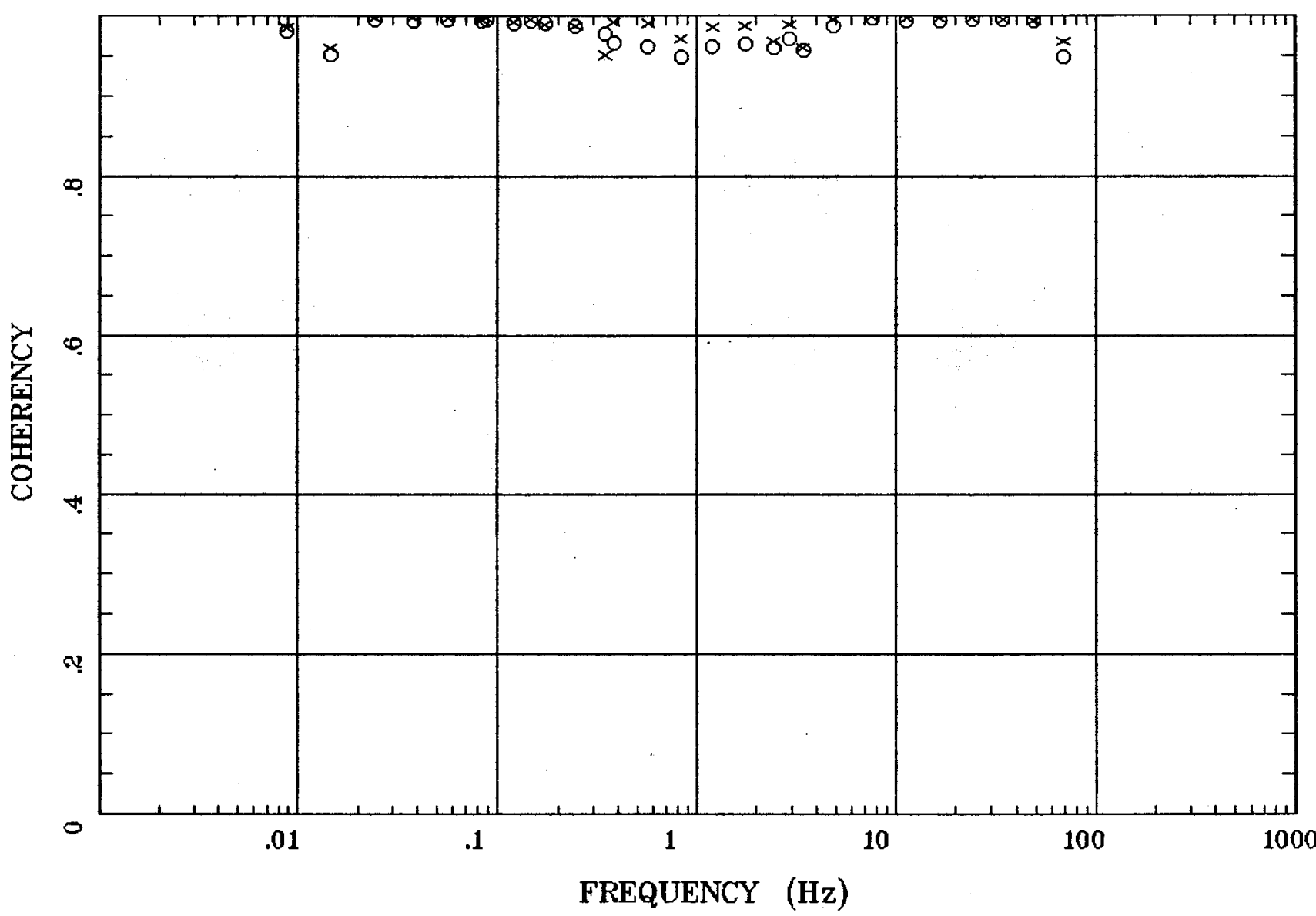

Client:

Remote: e-fld North $90 \mathrm{~m}$ Acquired: 10:4 Sep 16, 2003 Survey Co:USGS
Rotation:

Filename: ap18m.avg

Channels: Ch1 Ch2 Ch3 Ch4 Ch5 Ch6 Ch7 Plotted: 11:20 Sep 24, 2003

$<$ EMI - ElectroMagnetic Instruments 


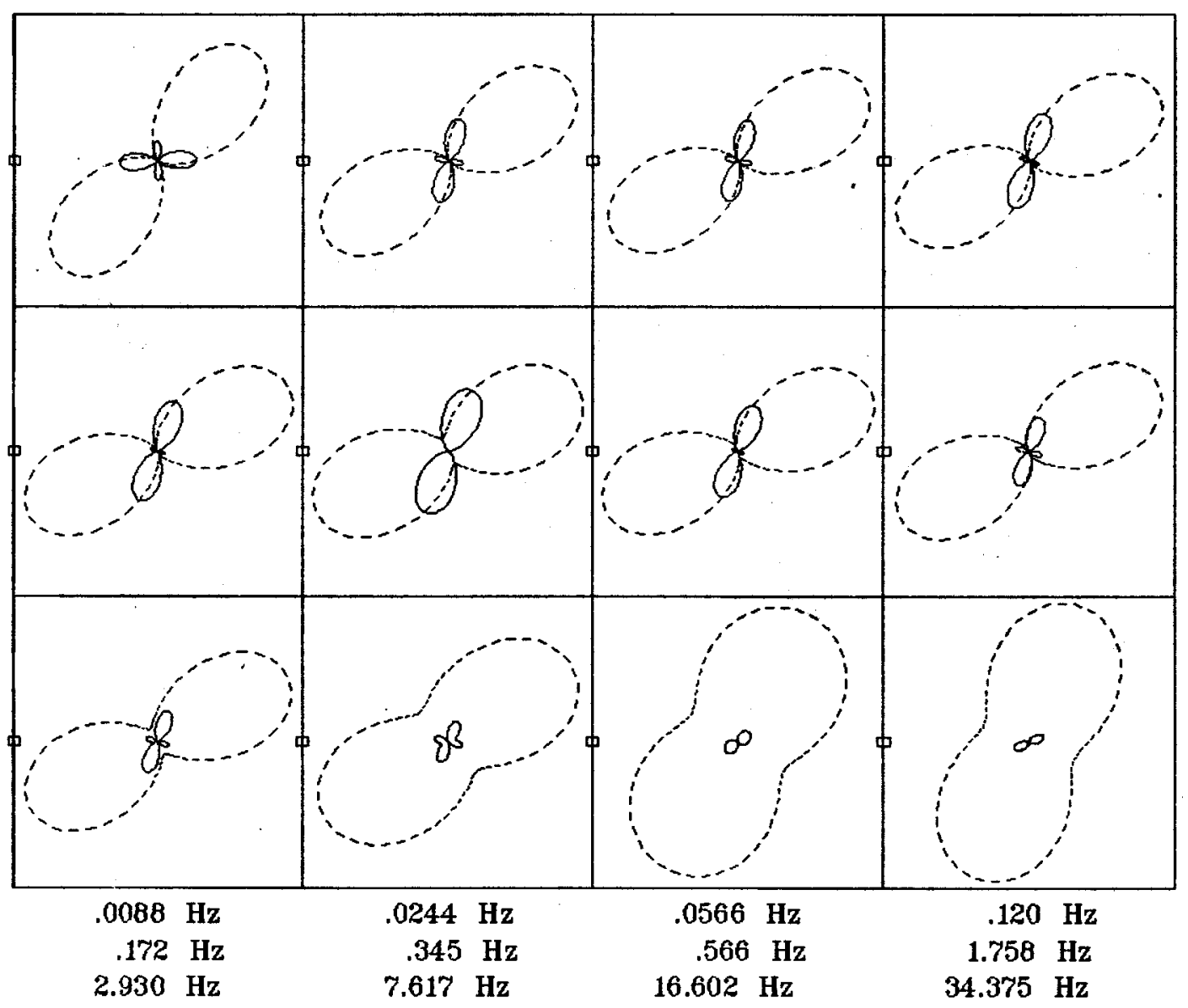

Rotation:

Client:

Remote: e-fld North $90 \mathrm{~m}$ Acquired: 10:4 Sep 16, 2003 Survey Co:USGS
Filename: ap18m.avg

Channels: Ch1 Ch2 Ch3 Ch4 Ch5 Ch6 Ch7 Plotted: 11:20 Sep 24, 2003

< EMI - ElectroMagnetic Instruments > 


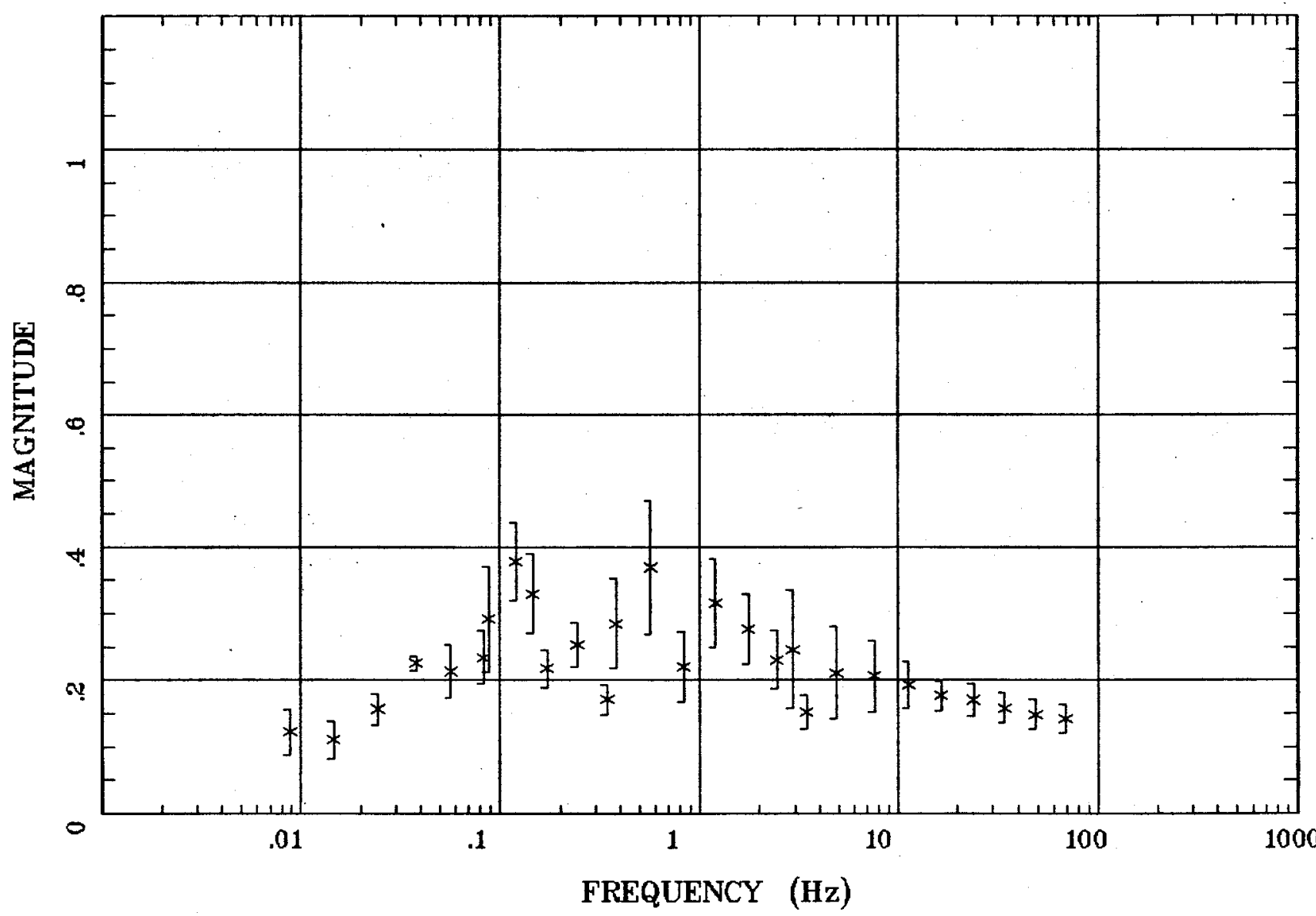

Client:

Remote: e-fld North $90 \mathrm{~m}$ Acquired: 10:4 Sep 16, 2003 Survey Co:USGS
Rotation:

Filename: ap18m.avg

Channels: Ch1 Ch2 Ch3 ch4 Ch5 Ch6 Ch7 Plotted: 11:20 Sep 24, 2003

$<$ EMI - ElectroMagnetic Instruments 


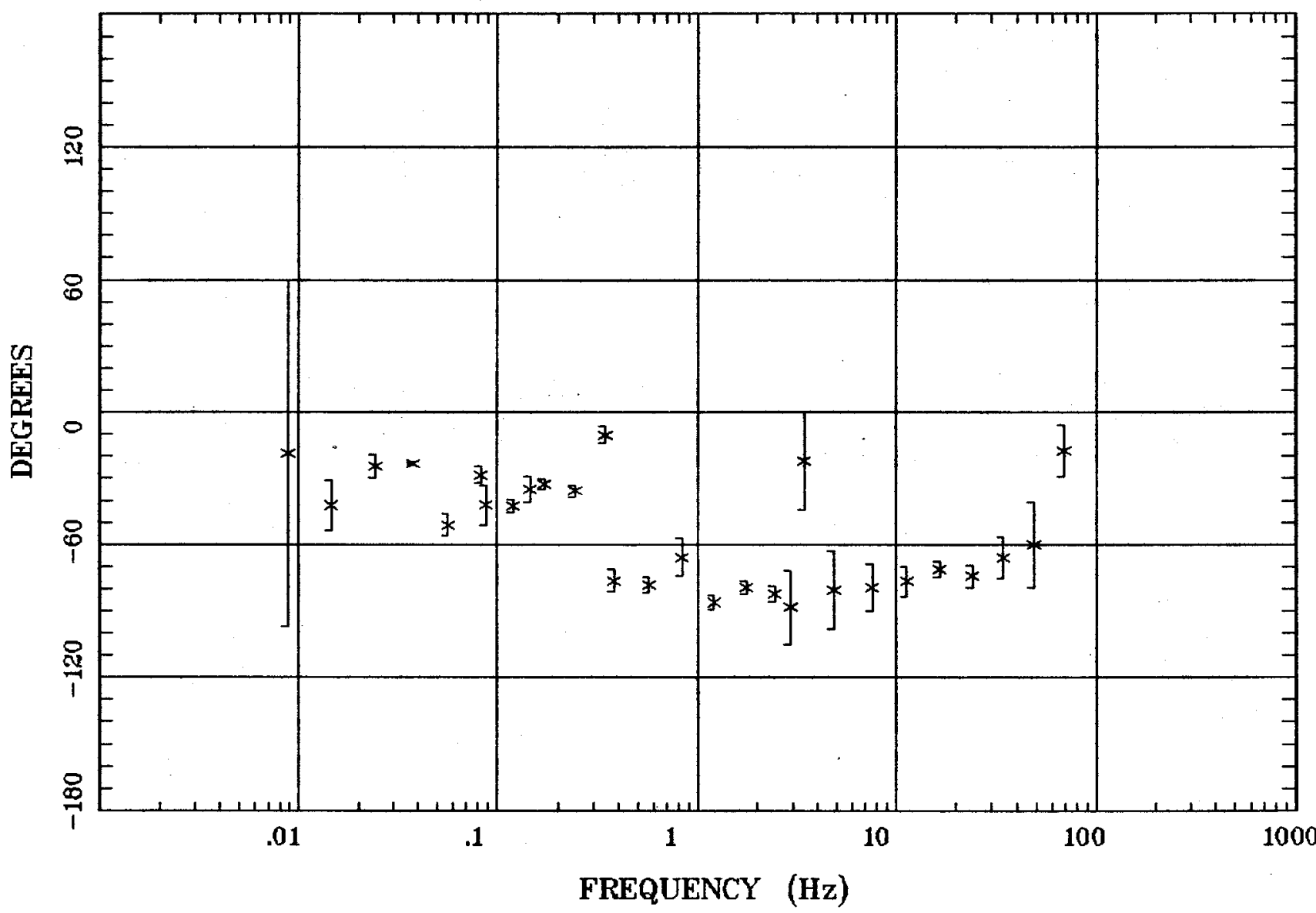

Client:

Remote: e-fld North $90 \mathrm{~m}$ Acquired: 10:4 Sep 16, 2003 Survey Co:USGS

\section{Rotation:}

Filename: ap18m.avg

Channels: Ch1 Ch2 Ch3 ch4 Ch5 Ch6 Ch7 Plotted: 11:20 Sep 24, 2003

$<$ EMI - ElectroMagnetic Instruments 


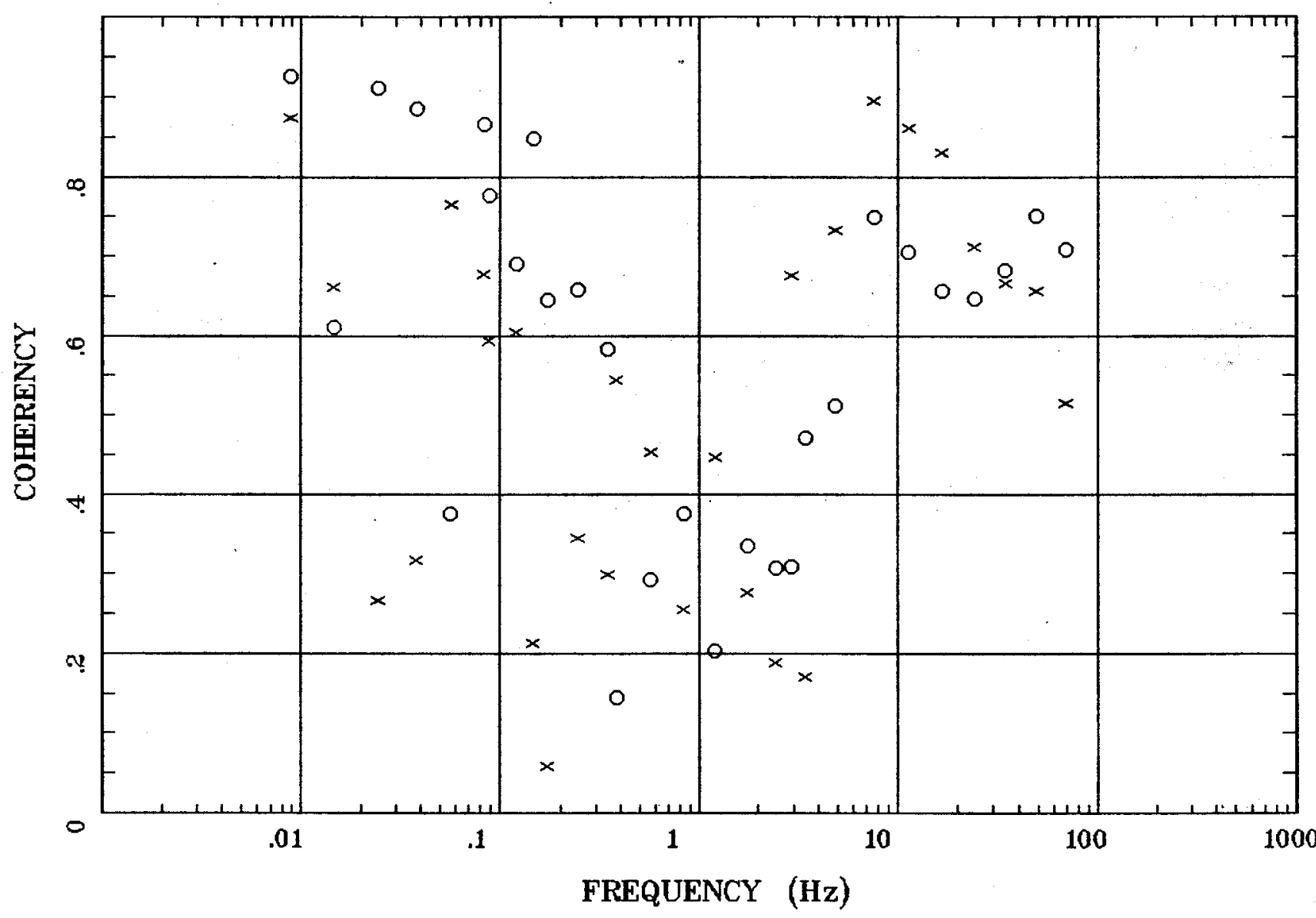

Client:

Remote: e-fld North $90 \mathrm{~m}$

Acquired: 10:4 Sep 16, 2003 Survey Co:USGS
Rotation:

Filename: ap18m.avg

Channels: Ch1 Ch2 Ch3 Ch4 Ch5 Ch6 Ch7 Plotted: 11:20 Sep 24, 2003

< EMI - ElectroMagnetic Instruments 


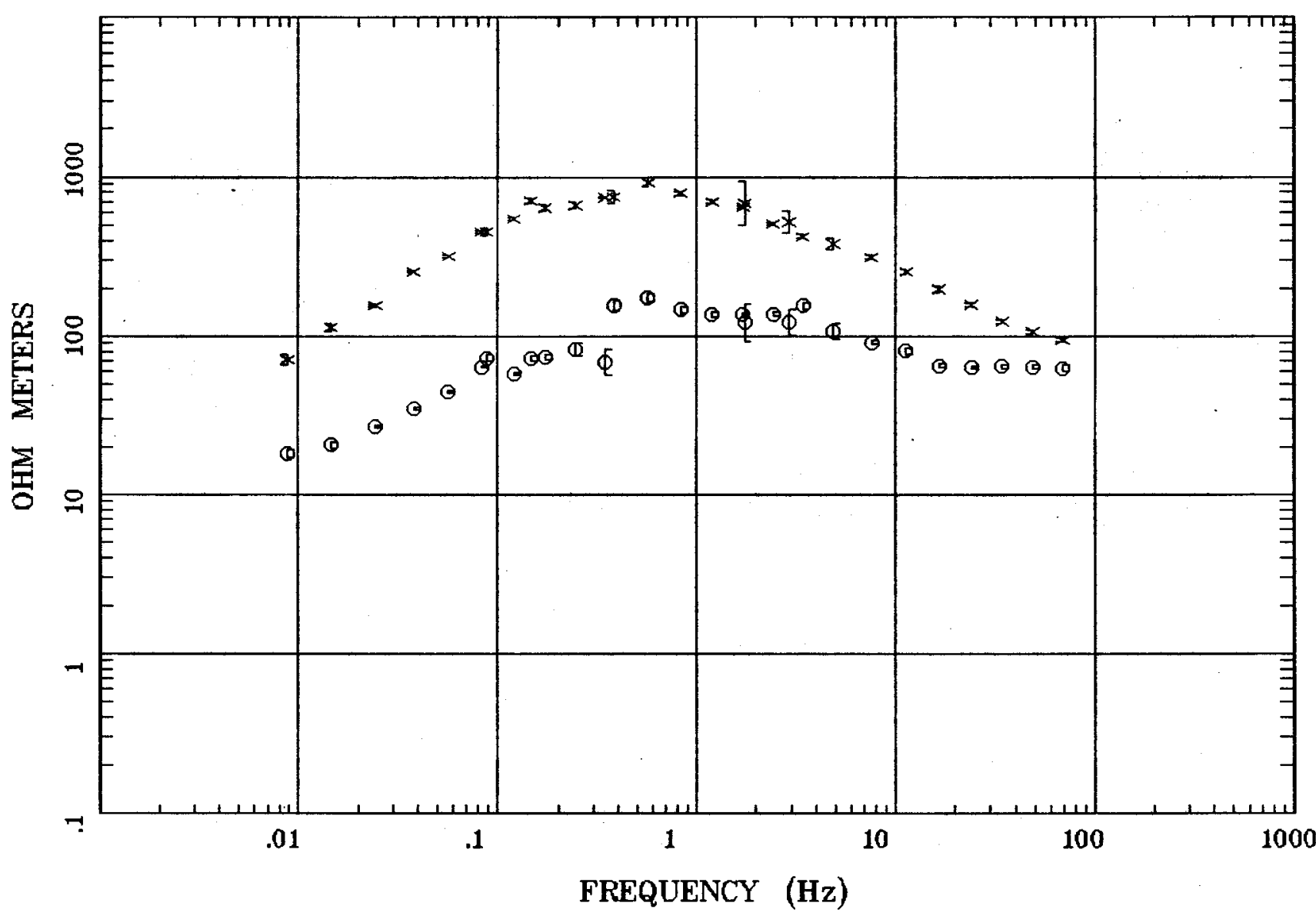

Client:

Remote: e-fld South $90 \mathrm{~m}$ Acquired: 14:2 Sep 16, 2003 Survey Co:USGS
Rotation:

Filename: ap19mall.avg Channels: Ch1 Ch2 Ch3 Ch4 Ch5 Ch6 Ch7 Platted: 11:20 Sep 24, 2003

$<$ EMI - ElectroMagnetic Instruments 
Kern Mts., NV $100 \mathrm{k} \quad$ Station 19

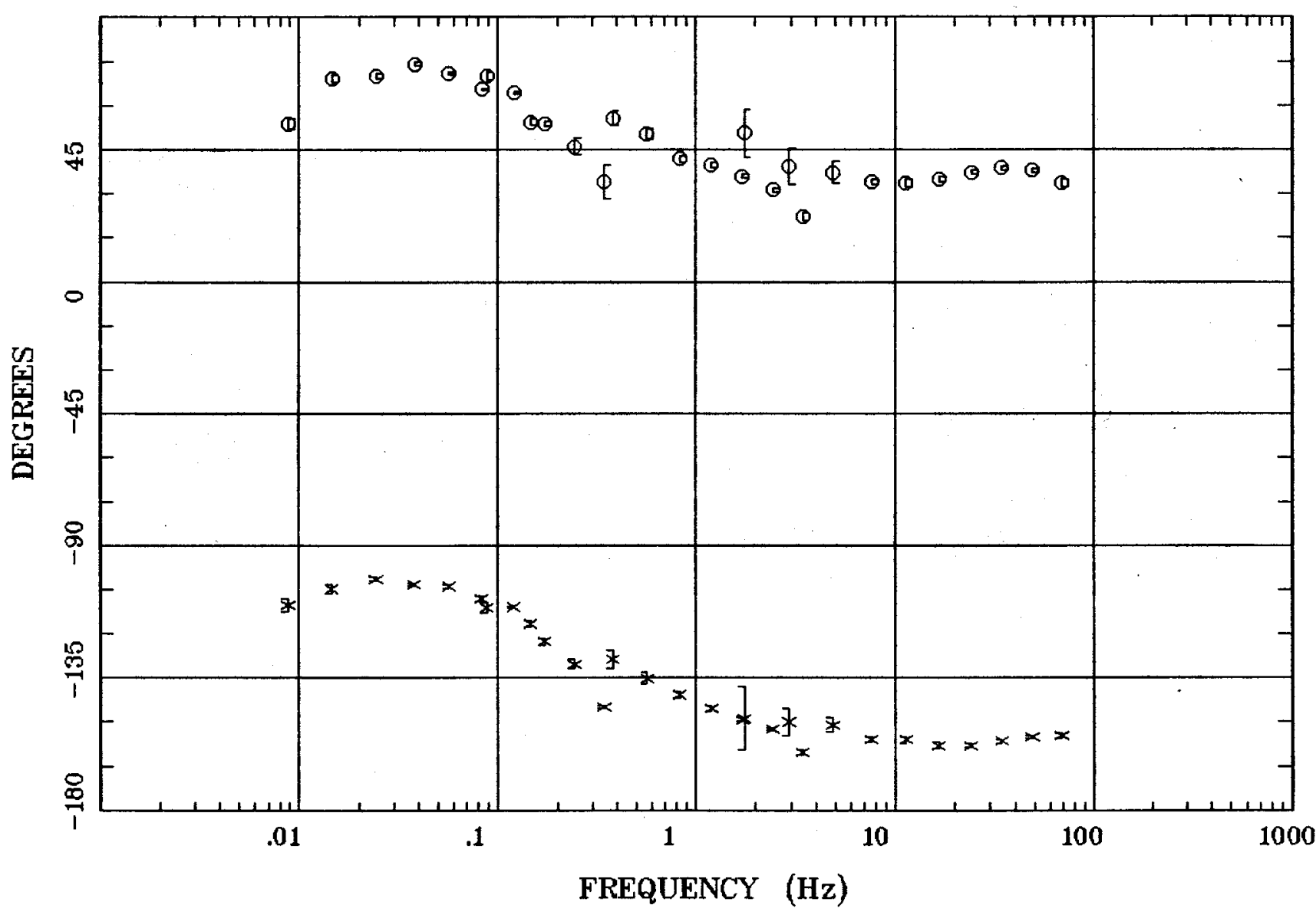

Client:

Remote: e-fld South $90 \mathrm{~m}$ Acquired: 14:2 Sep 16, 2003 Survey Co:USGS
Rotation:

Filename: ap19mall.avg Channels: Ch1 Ch2 Ch3 Ch4 Ch5 Ch6 Ch7 Plotted: 11:20 Sep 24, 2003

$<$ EMI - ElectroMagnetic Instruments 


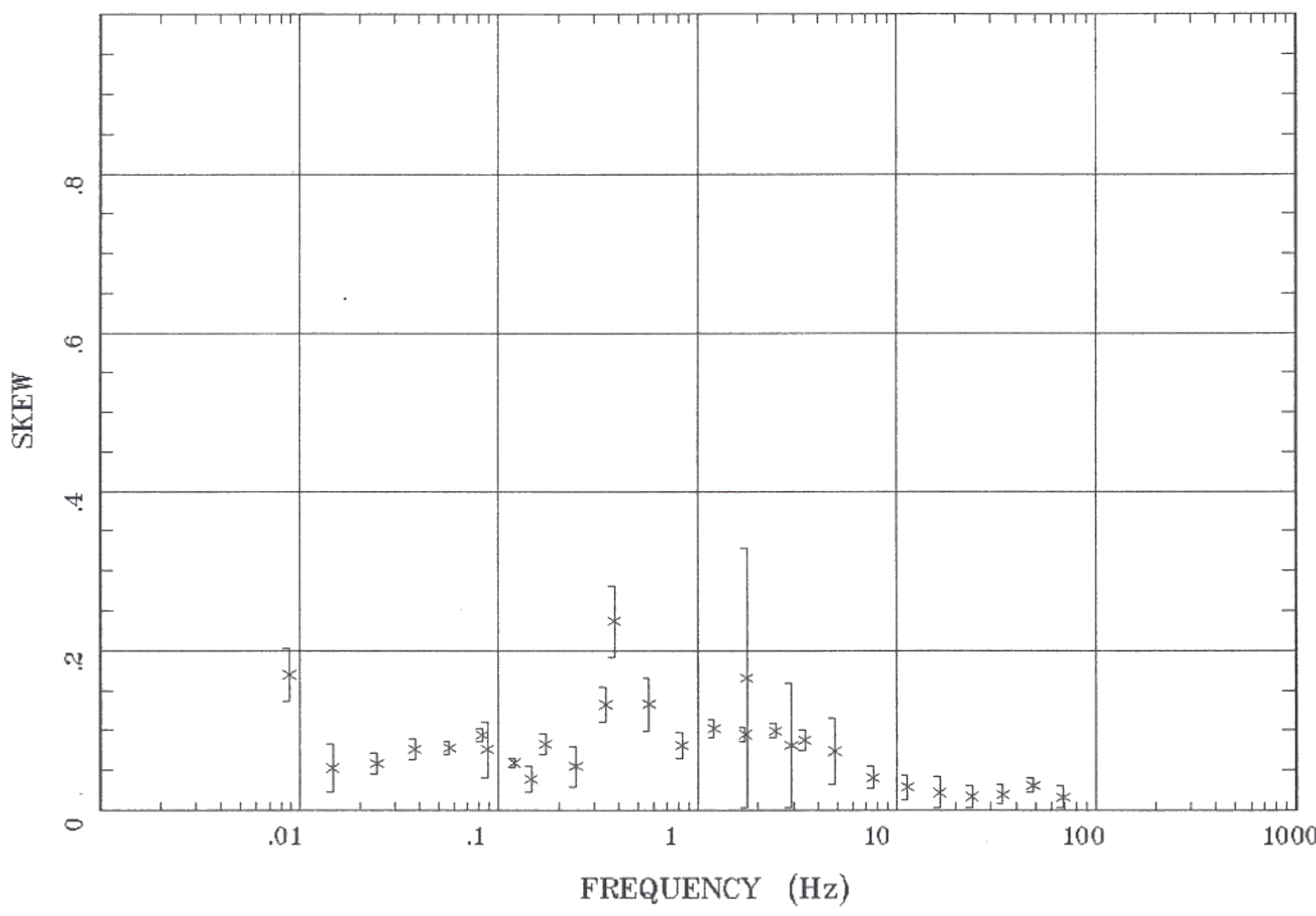

Client:

Remote: e-fld South 90m Acquired: 14:2 Sep 16, 2003 Survey Co:USGS

\section{Rotation:}

Filename: ap19mall.avg

Channels: Ch1 Ch2 Ch3 ch4 ch5 ch6 Ch7 Plotted: 11:20 Sep 24, 2003

$<$ EMI - ElectroMagnetic Instruments 
E MULT Coh.

Kern Mts., NV 100k Station 19

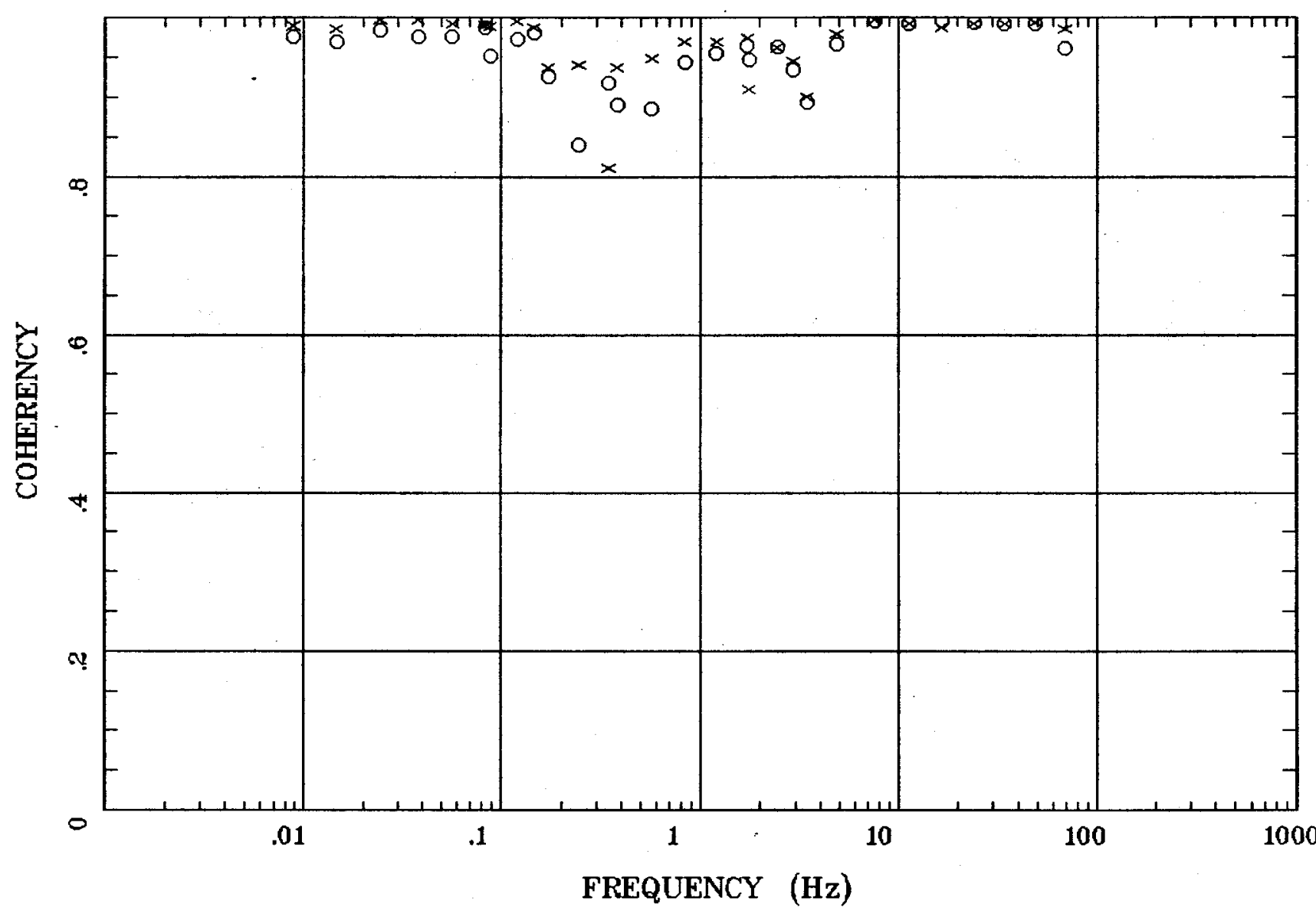

Client:

Remote: e-fld South $90 \mathrm{~m}$

Acquired: 14:2 Sep 16, 2003 Survey Co:USGS
Rotation:

Filename: ap19mall.avg

Channels: Ch1 Ch2 Ch3 Ch4 Ch5 Ch6 Ch7

Plotted: 11:21 Sep 24, 2003

$<$ EMI - ElectroMagnetic Instruments 


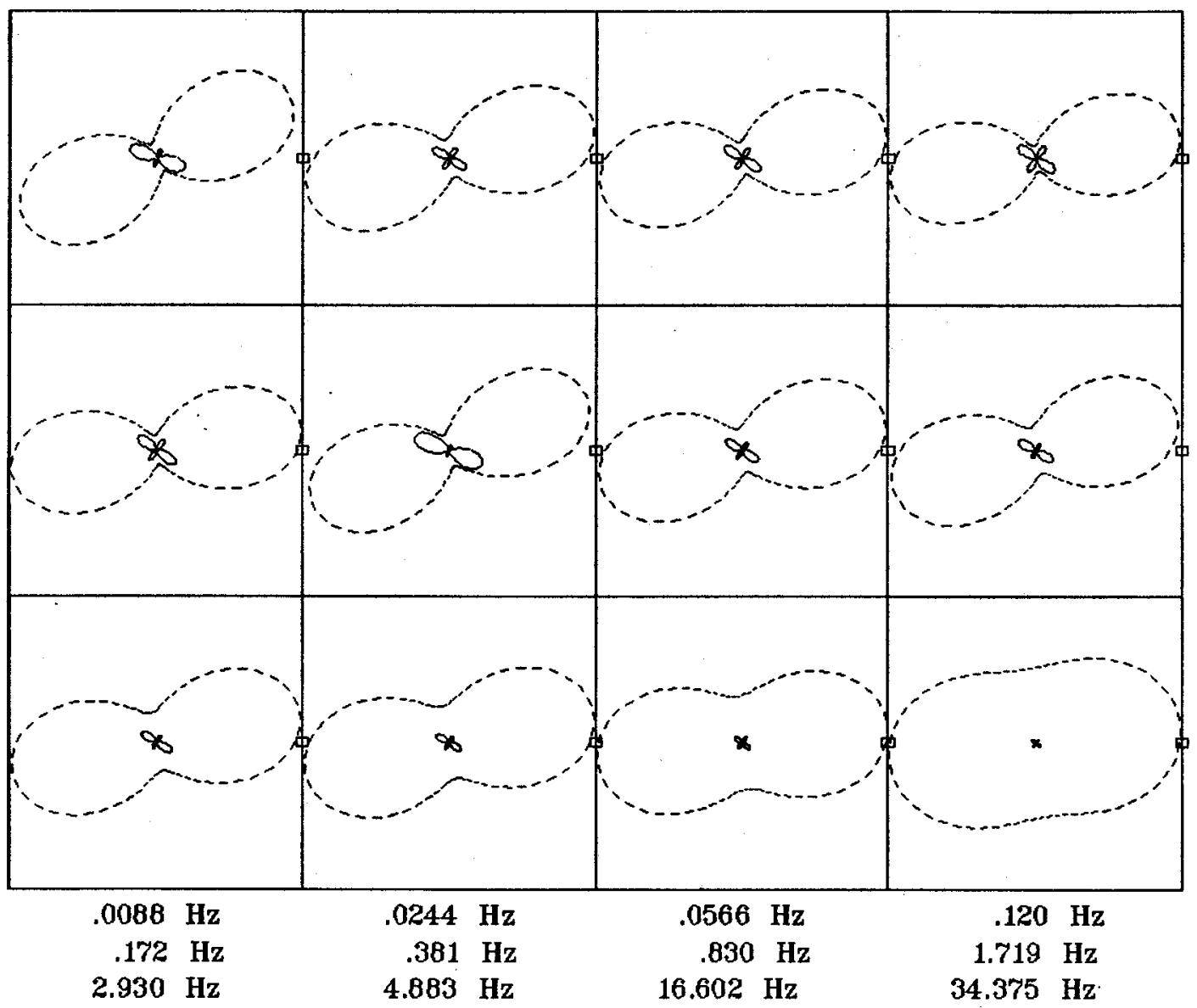

Client:

Remote: e-fld South $90 \mathrm{~m}$ Acquired: 14:2 Sep 16, 2003 Survey Co:USGS
Rotation:

Filename: ap19mall.avg

Channels: Ch1 Ch2 Ch3 ch4 Ch5 Ch6 Chr Plotted: 11:21 Sep 24, 2003

< EMI - ElectroMagnetic Instruments > 


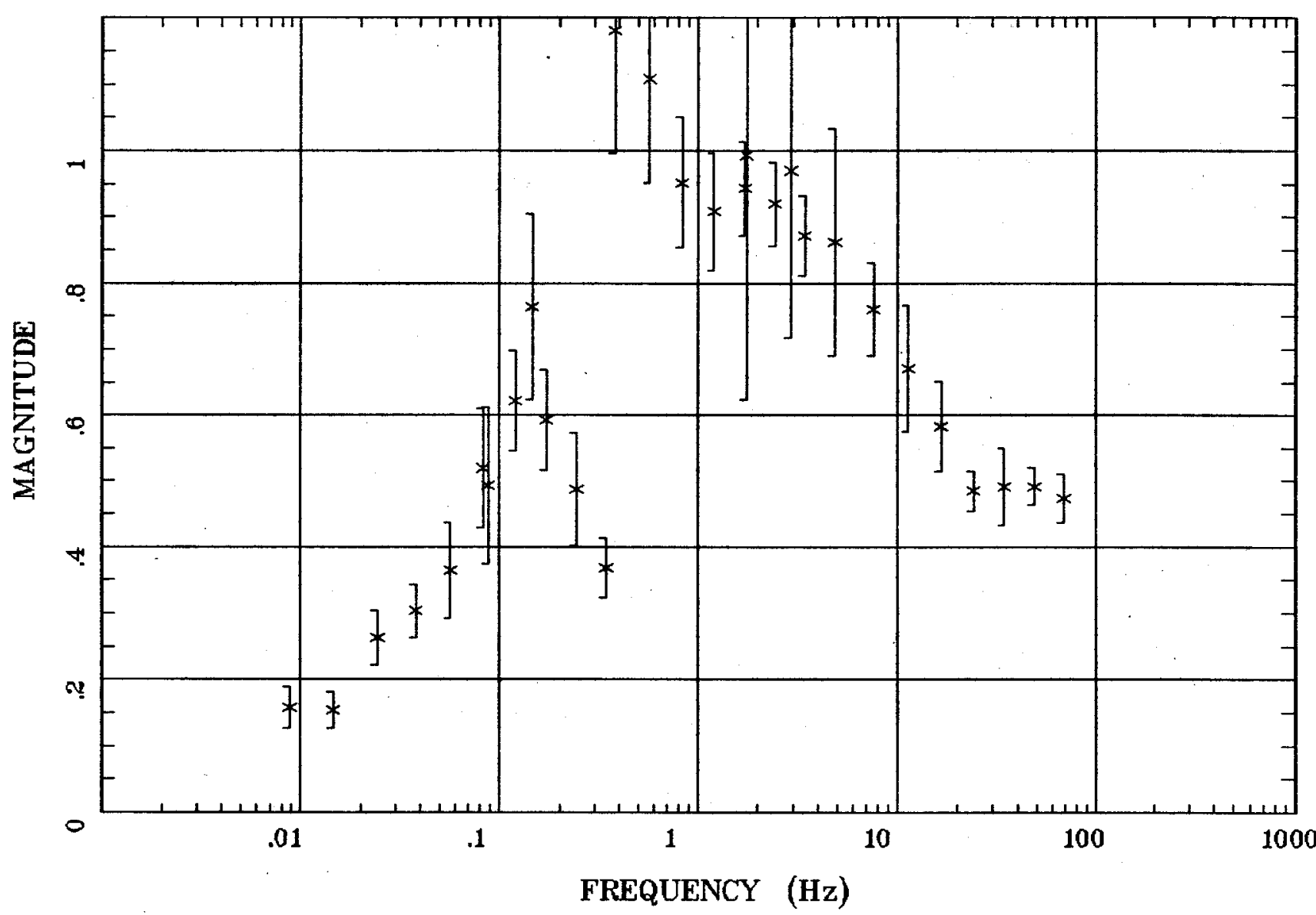

Client:

Remote: e-fld South $90 \mathrm{~m}$ Acquired: 14:2 Sep 16, 2003 Survey Co:USGS

\section{Rotation:}

Filename: ap19mall.avg

Channels: Ch1 Ch2 Ch3 Ch4 Ch5 Ch6 Ch7 Plotted: 11:21 Sep 24, 2003

$<$ EMI - ElectroMagnetic Instruments 


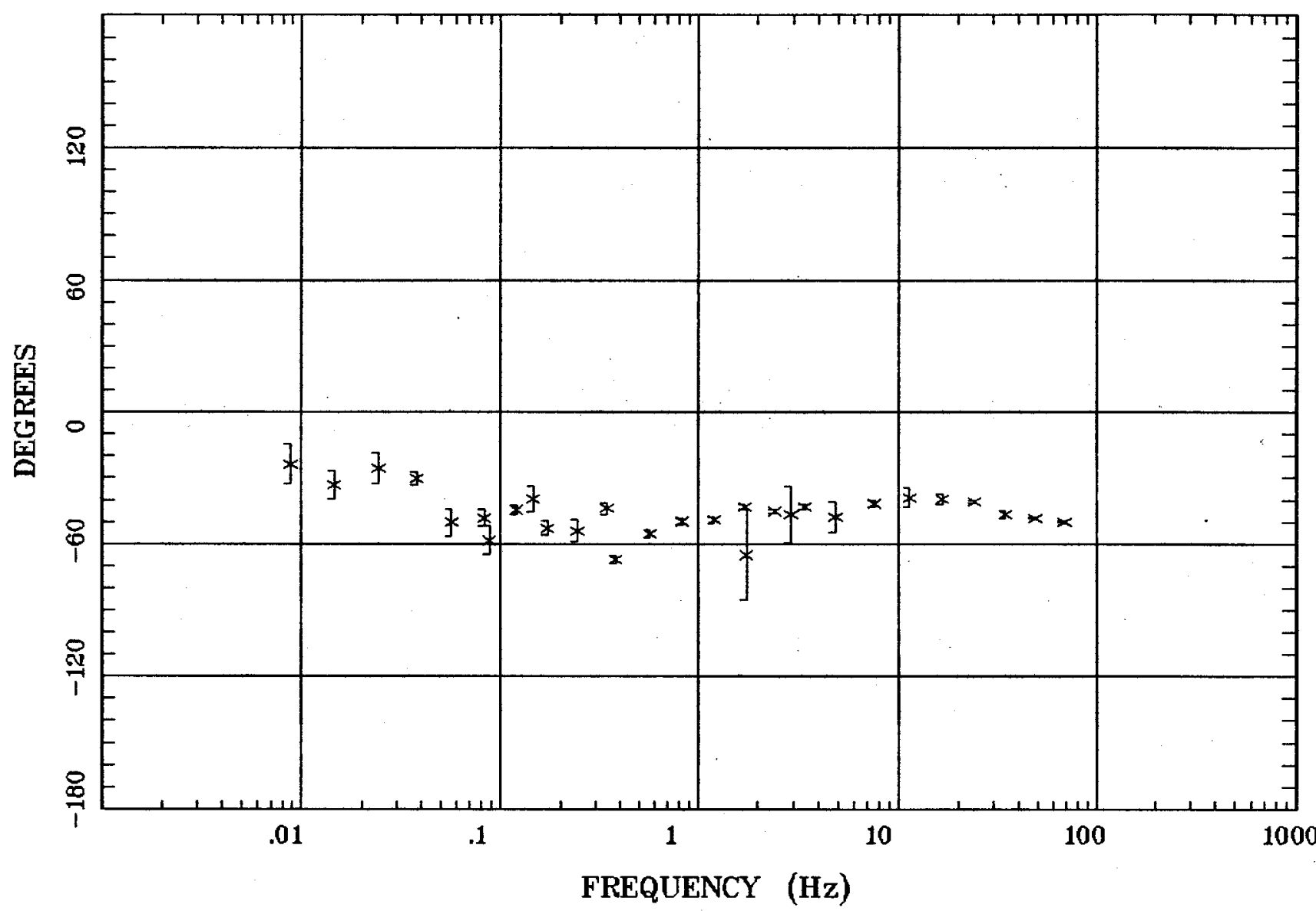

Client:

Remote: e-fld South $90 \mathrm{~m}$

Acquired: 14:2 Sep 16, 2003 Survey Co:USGS
Rotation:

Filename: ap19mall.avg

Channels: Ch1 Ch2 Ch3 Ch4 Ch5 Ch6 Ch7

Plotted: 11:21 Sep 24, 2003

< EMI - ElectroMagnetic Instruments > 


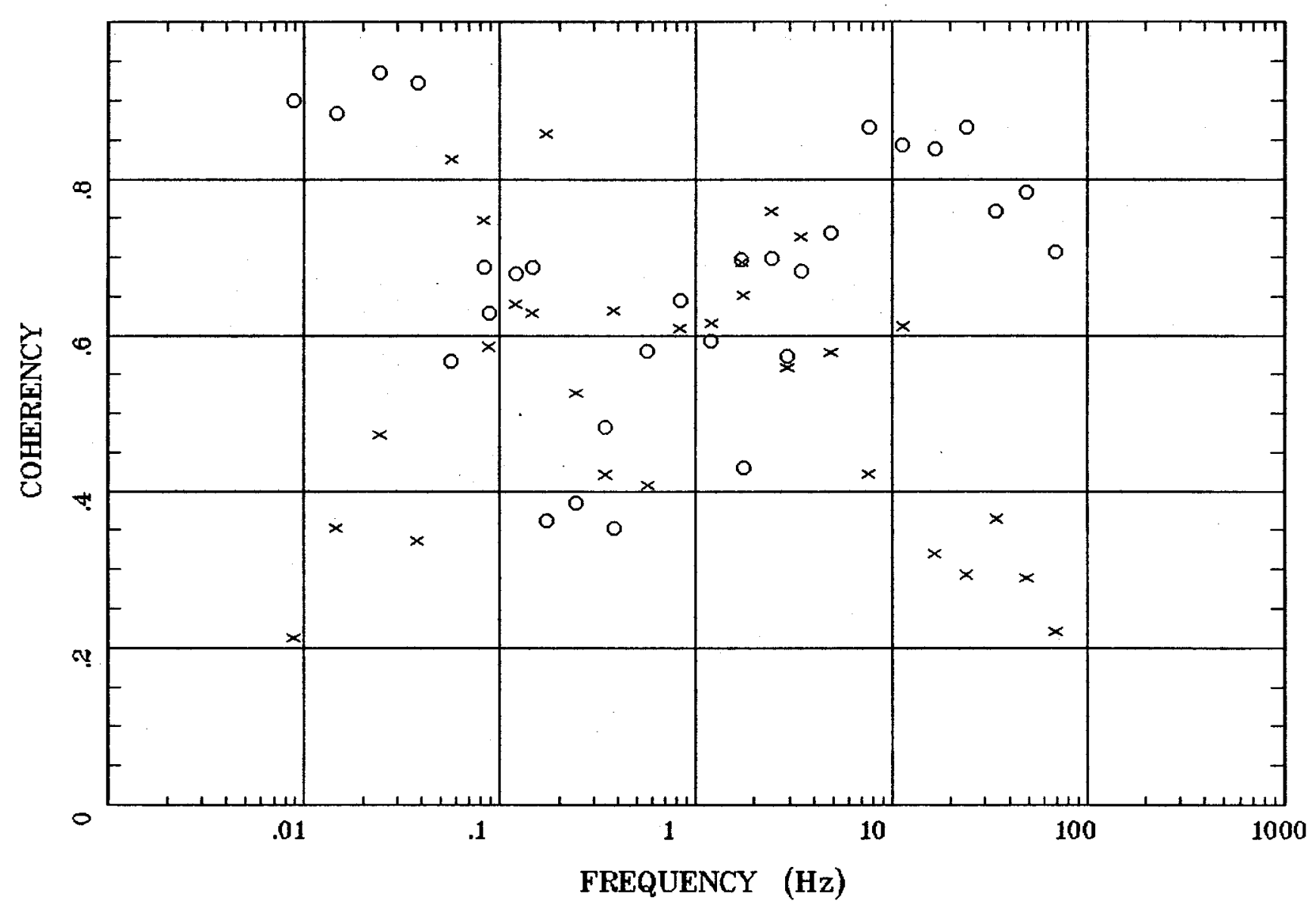

Client:

Remote: e-fld South $90 \mathrm{~m}$ Acquired: $14: 2 \operatorname{Sep} 16,2003$ Survey Co:USGS
Rotation:

Filename: ap19mall.avg

Channels: Ch1 Ch2 Ch3 Ch4 Ch5 Ch6 Ch7

Plotted: 11:21 Sep 24, 2003

< EMI - ElectroMagnetic Instruments 


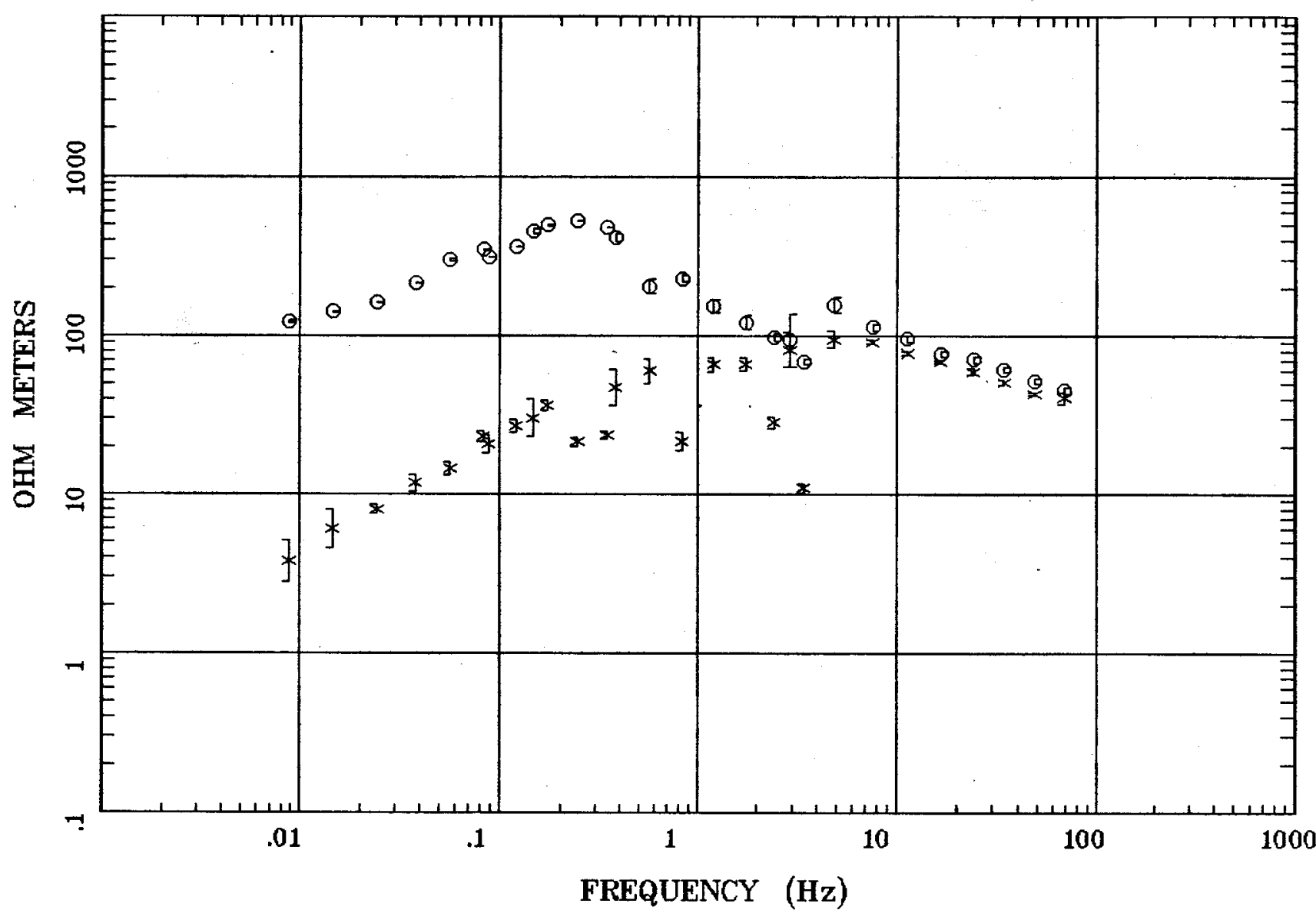

Client:

Remote: e-fld E $90 \mathrm{~m}$

Acquired: 10:4 Sep 17, 2003

Survey Co:USGS
Rotation:

Filename: ap20m.avg

Channels: Ch1 Ch2 Ch3 Ch4 Ch5 Ch6 Ch7

Plotted: 11:21 Sep 24, 2003

$<$ EMI - ElectroMagnetic Instruments 


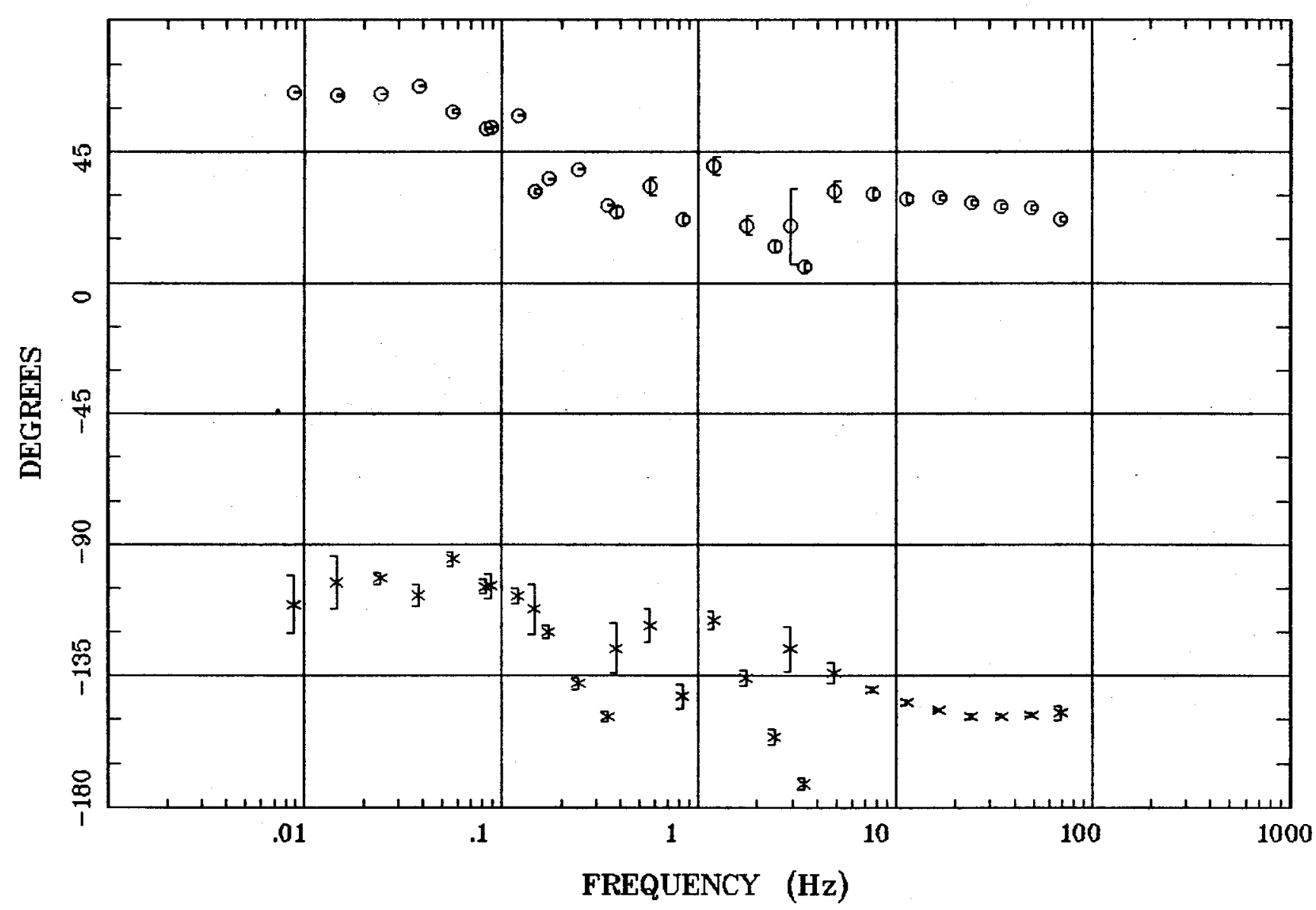

Client:

Remote: e-fld E $90 \mathrm{~m}$

Acquired: 10:4 Sep 17, 2003 Survey Co:USGS
Rotation:

Filename: ap20m.avg

Channels: Ch1 Ch2 Ch3 Ch4 Ch5 Ch6 Chr

Plotted: 11:21 Sep 24, 2003

<EMI - ElectroMagnetic Instruments > 


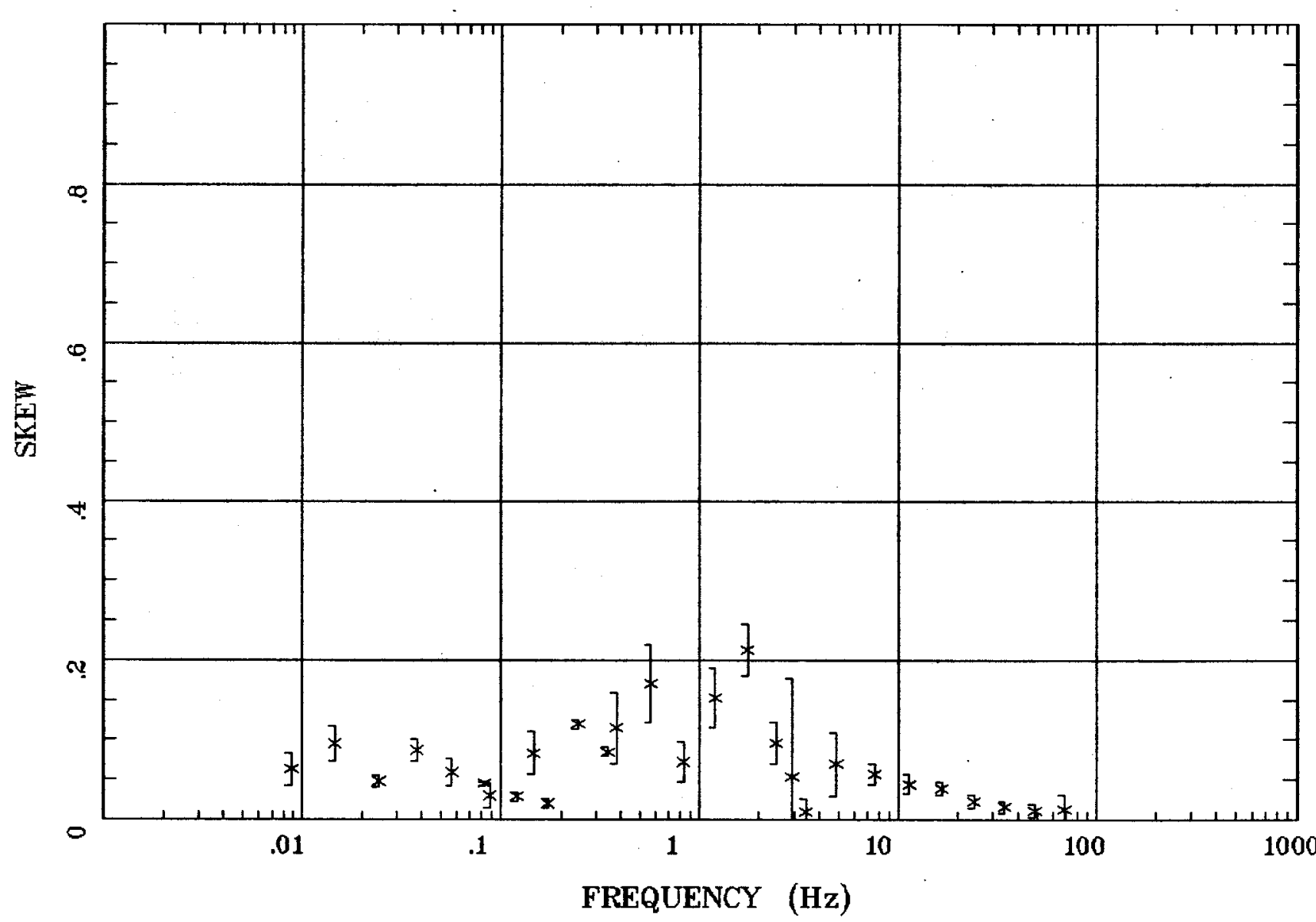

Client:

Remote: e-fld E $90 \mathrm{~m}$ Acquired: 10:4 Sep 17, 2003 Survey Co:USGS
Rotation:

Filename: aprom.avg

Channels: Ch1 Ch2 Ch3 Ch4 Ch5 Ch6 Ch7 Plotted: 11:21 Sep 24, 2003

$<$ EMI - ElectroMagnetic Instruments 
E MULT Coh.

Ely, NV 100k

Station 20

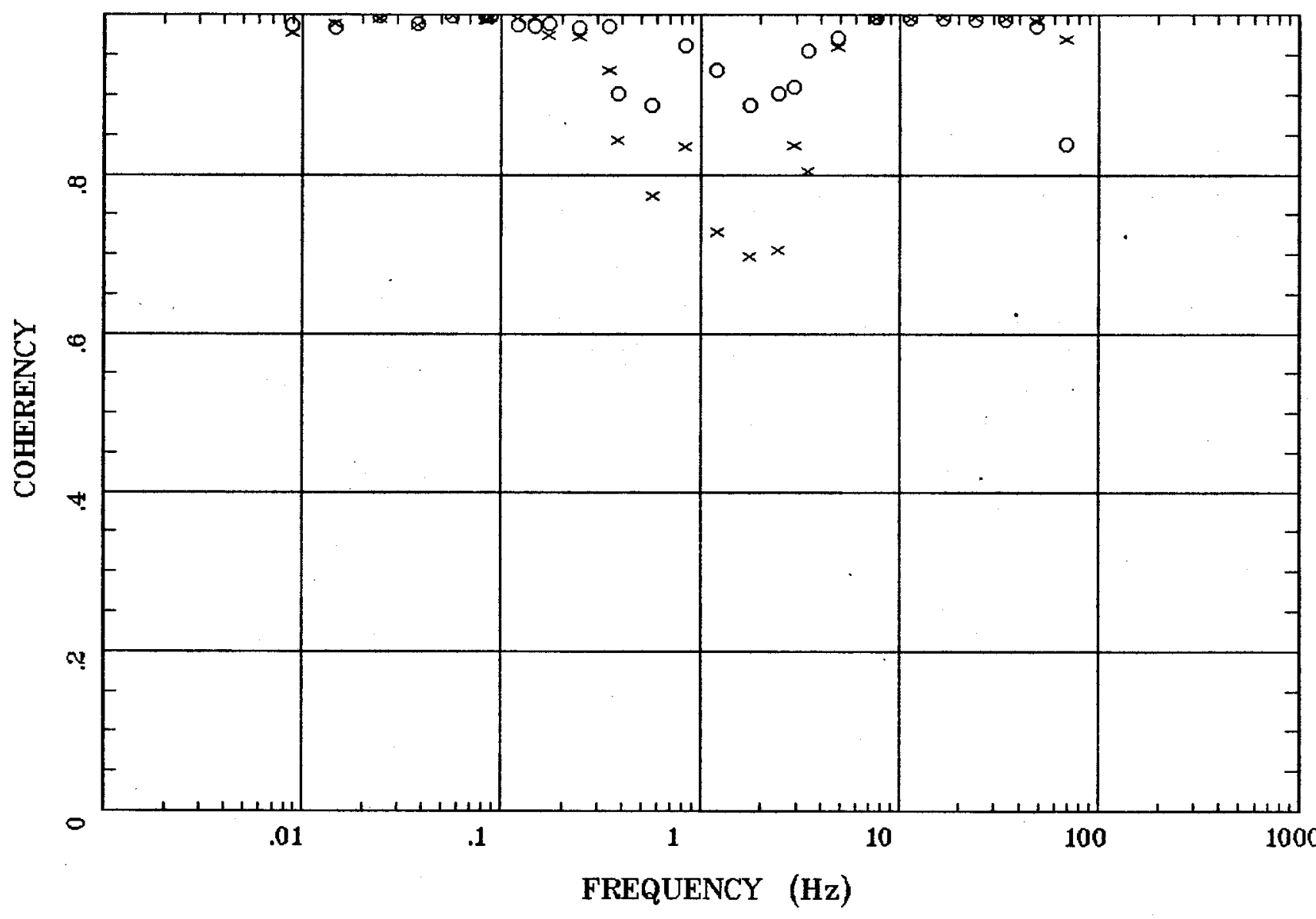

Client:

Remote: e-fld E $90 \mathrm{~m}$

Acquired: 10:4 Sep 17, 2003

Survey Co:USGS
Rotation:

Filename: ap20m.avg

Channels: Ch1 Ch2 Ch3 Ch4 Ch5 Ch6 Ch7

Plotted: 11:21 Sep 24, 2003

< EMI - ElectroMagnetic Instruments 


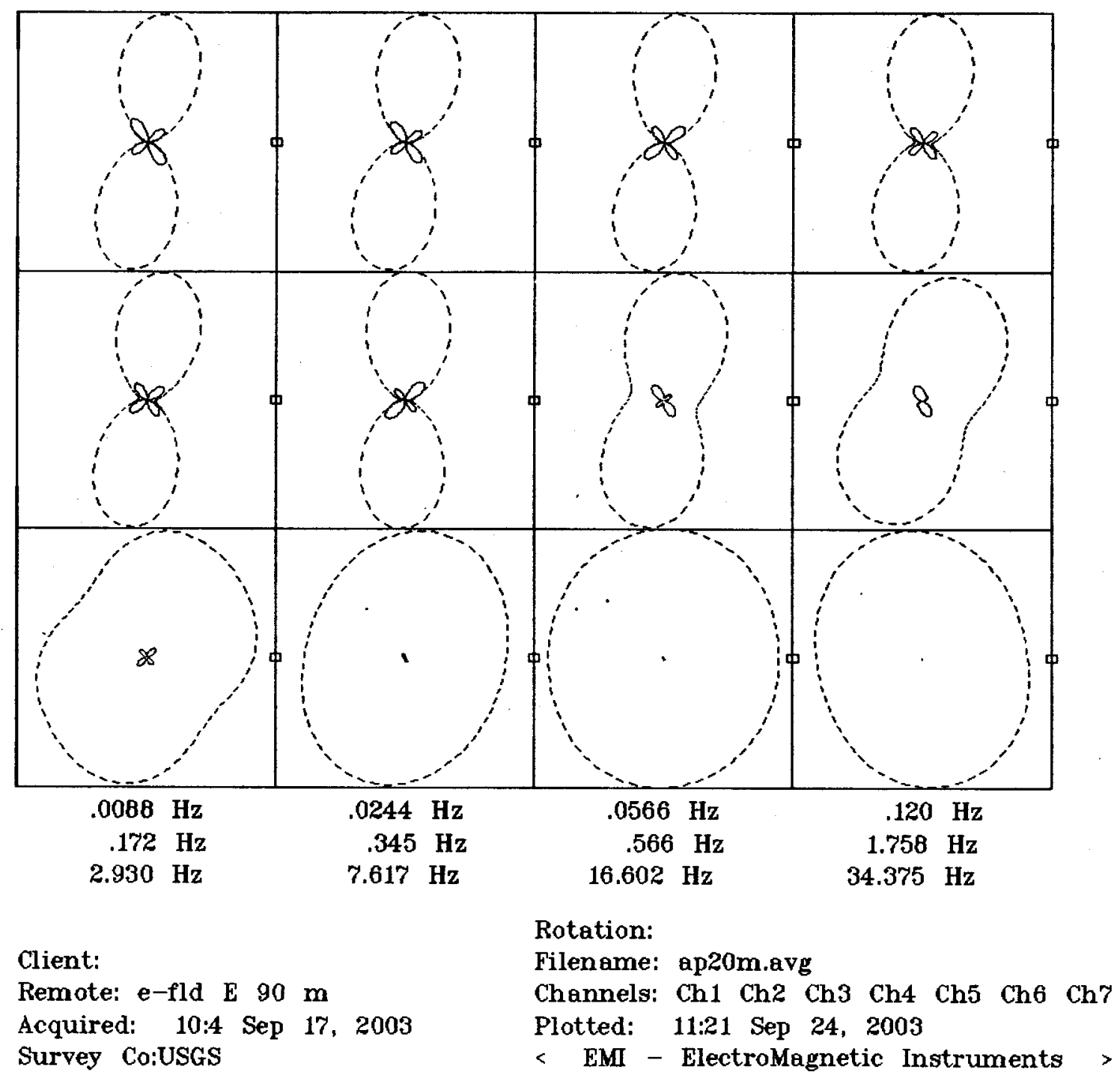




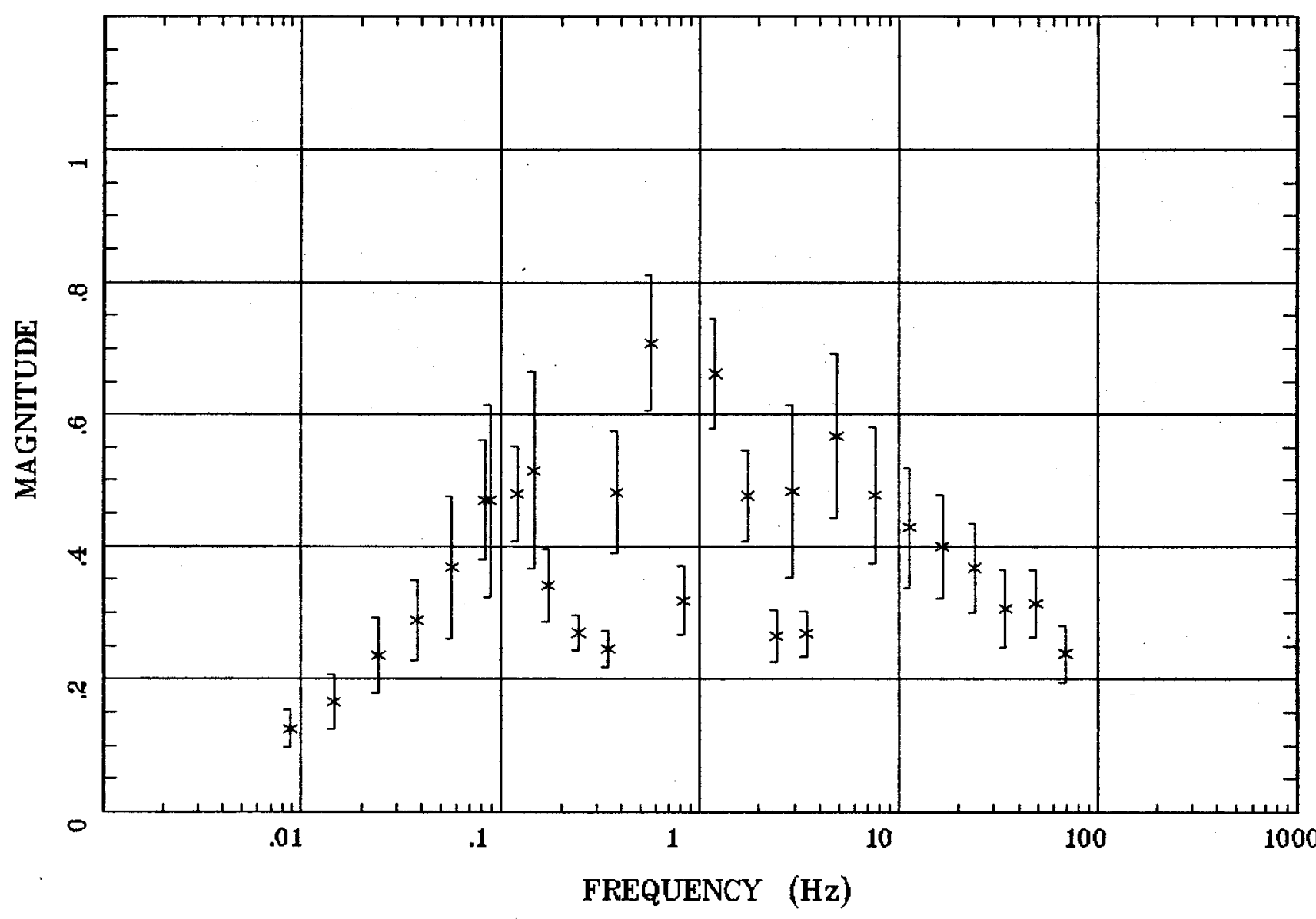

Client:

Remote: e-fld E $90 \mathrm{~m}$

Acquired: 10:4 Sep 17, 2003

Survey Co:USGS
Rotation:

Filename: ap20m.avg

Channels: Ch1 Ch2 Ch3 Ch4 Ch5 Ch6 Ch7

Plotted: 11:21 Sep 24, 2003

$<$ EMI - ElectroMagnetic Instruments 


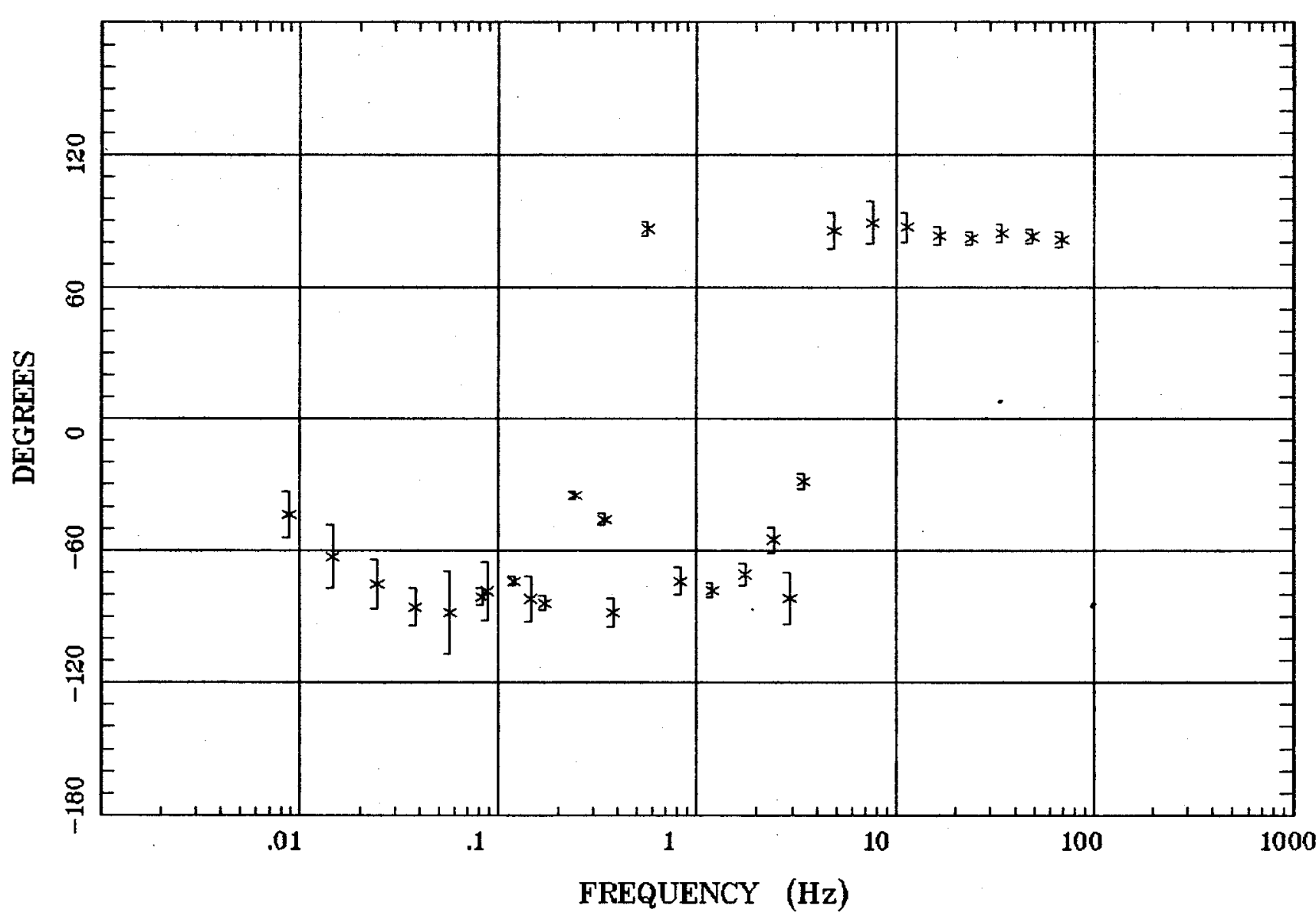

Client:

Remote: e-fld E $90 \mathrm{~m}$

Acquired: 10:4 Sep 17, 2003

Survey Co:USGS
Rotation:

Filename: ap20m.avg

Channels: Ch1 Ch2 Ch3 Ch4 Ch5 Ch6 Ch7 Plotted: 11:21 Sep 24, 2003

$<$ EMI - ElectroMagnetic Instruments > 


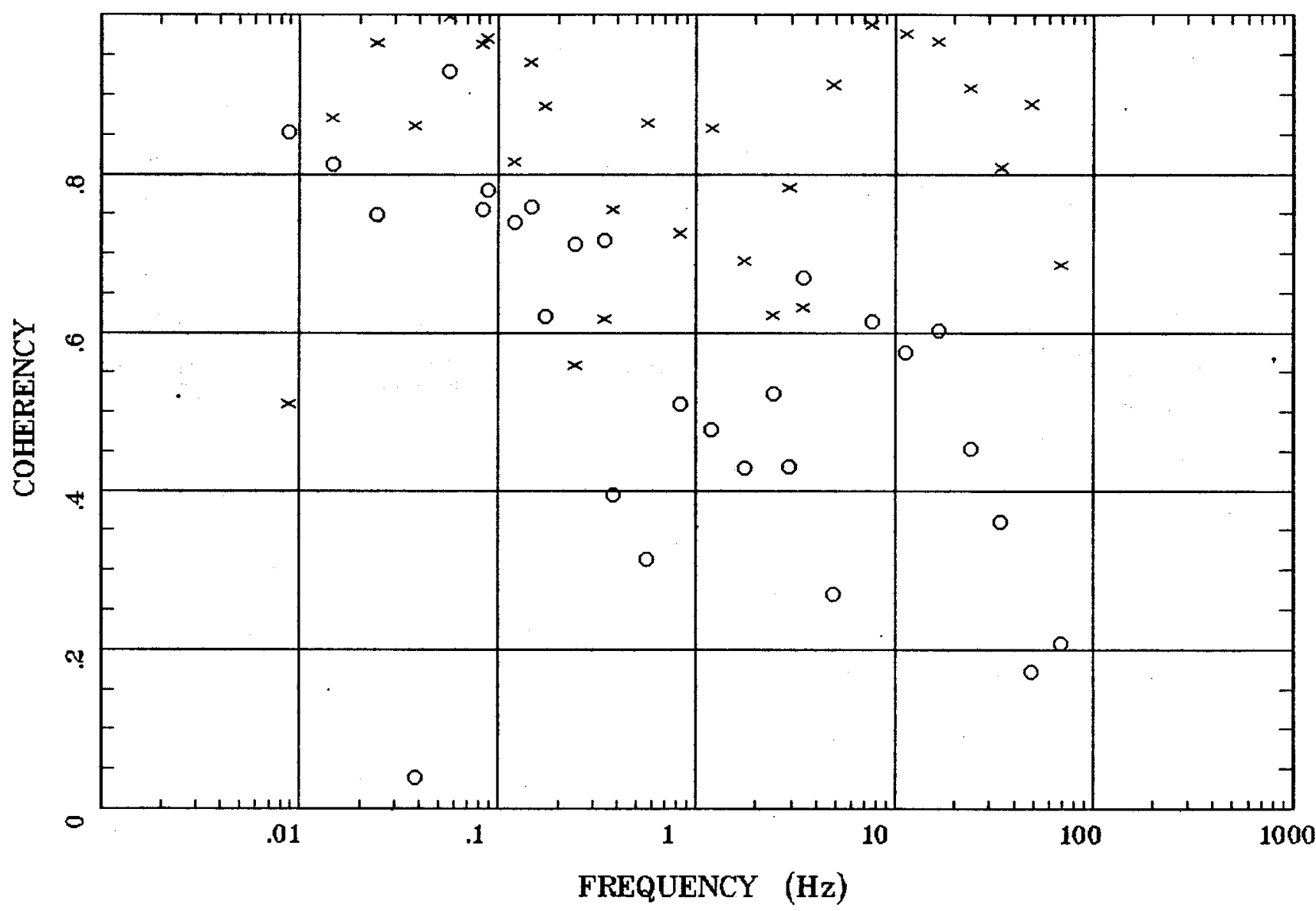

Client:

Remote: e-fld E $90 \mathrm{~m}$ Acquired: 10:4 Sep 17, 2003 Survey Co:USGS
Rotation:

Filename: ap20m.avg

Channels: Ch1 Ch2 Ch3 Ch4 Ch5 Ch6 Ch7 Plotted: 11:22 Sep 24, 2003

$<$ EMI - ElectroMagnetic Instruments 


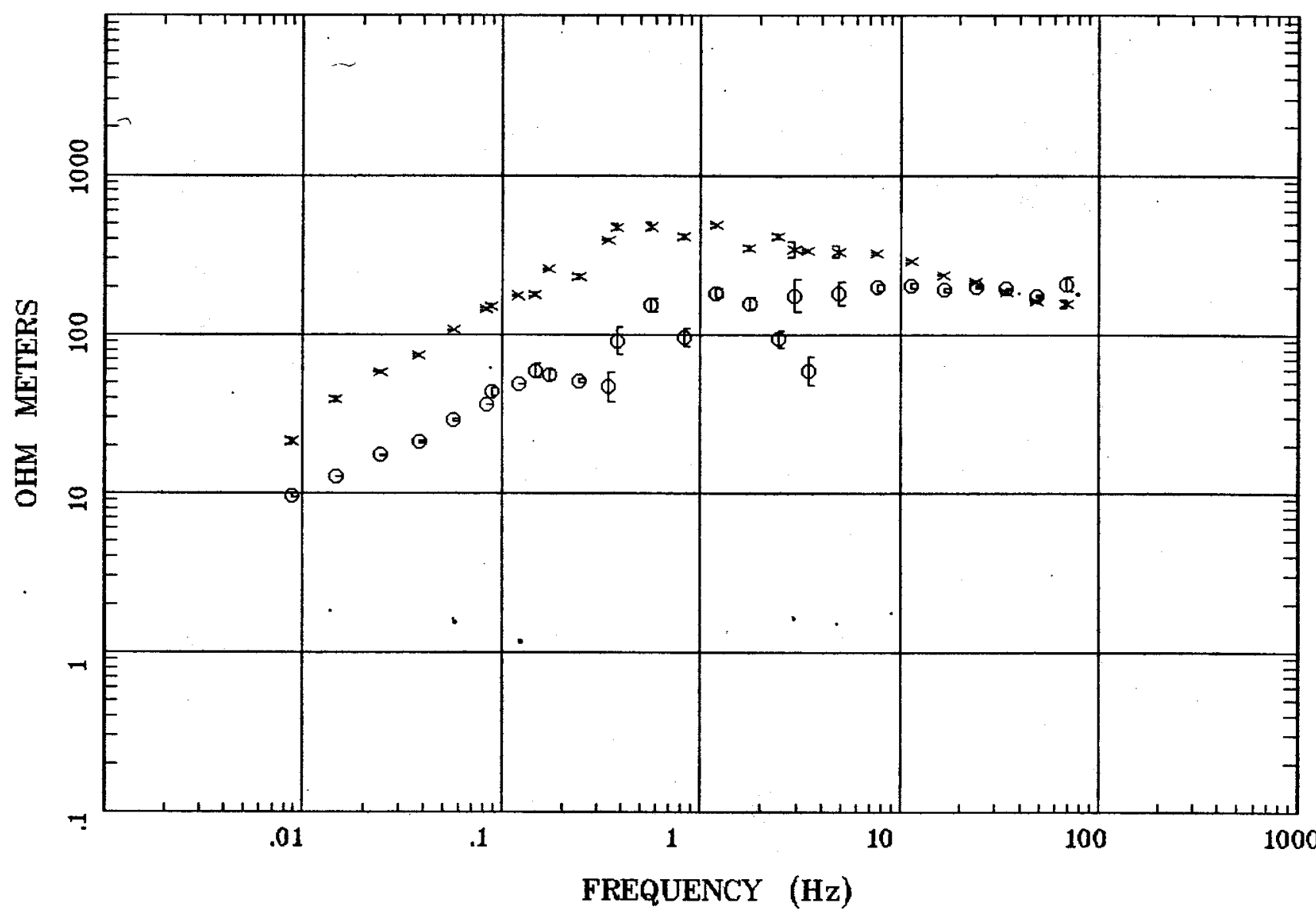

Client:

Remote: e-fld E $90 \mathrm{~m}$

Acquired: 14:4 Sep 17, 2003

Survey Co:USGS
Rotation:

Filename: ap21m.avg

Channels: Ch1 Ch2 Ch3 Ch4 Ch5 Ch6 Ch7

Platted: 14:17 Sep 24, 2003

$<$ EMI - ElectroMagnetic Instruments 


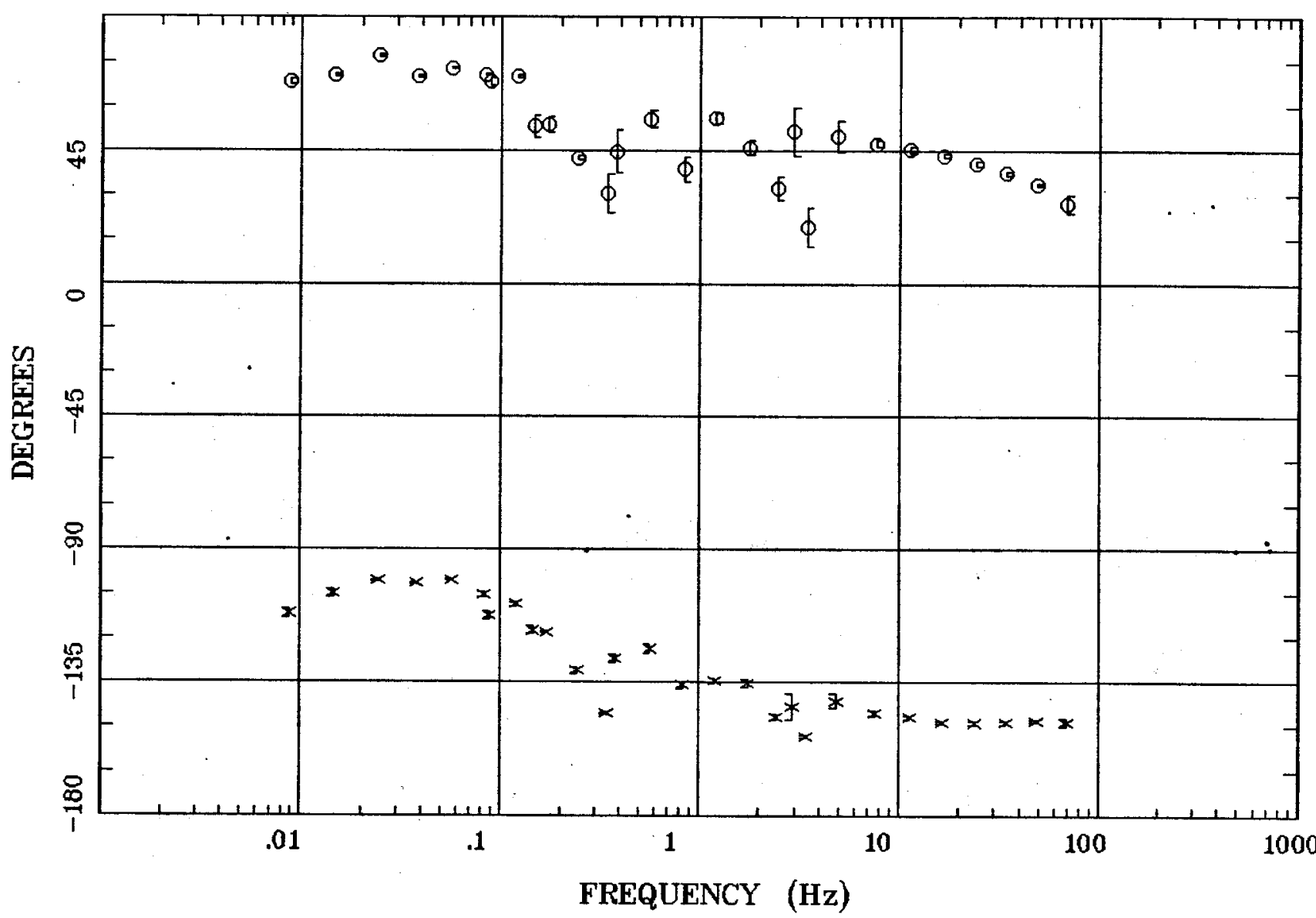

Client:

Remote: e-fld E $90 \mathrm{~m}$ Acquired: 14:4 Sep 17, 2003 Survey Co:USGS
Rotation:

Filename: ap21m.avg

Channels: Ch1 Ch2 Ch3 Ch4 Ch5 Ch6 Ch7 Plotted: 14:17 Sep 24, 2003

< EMI - ElectroMagnetic Instruments 


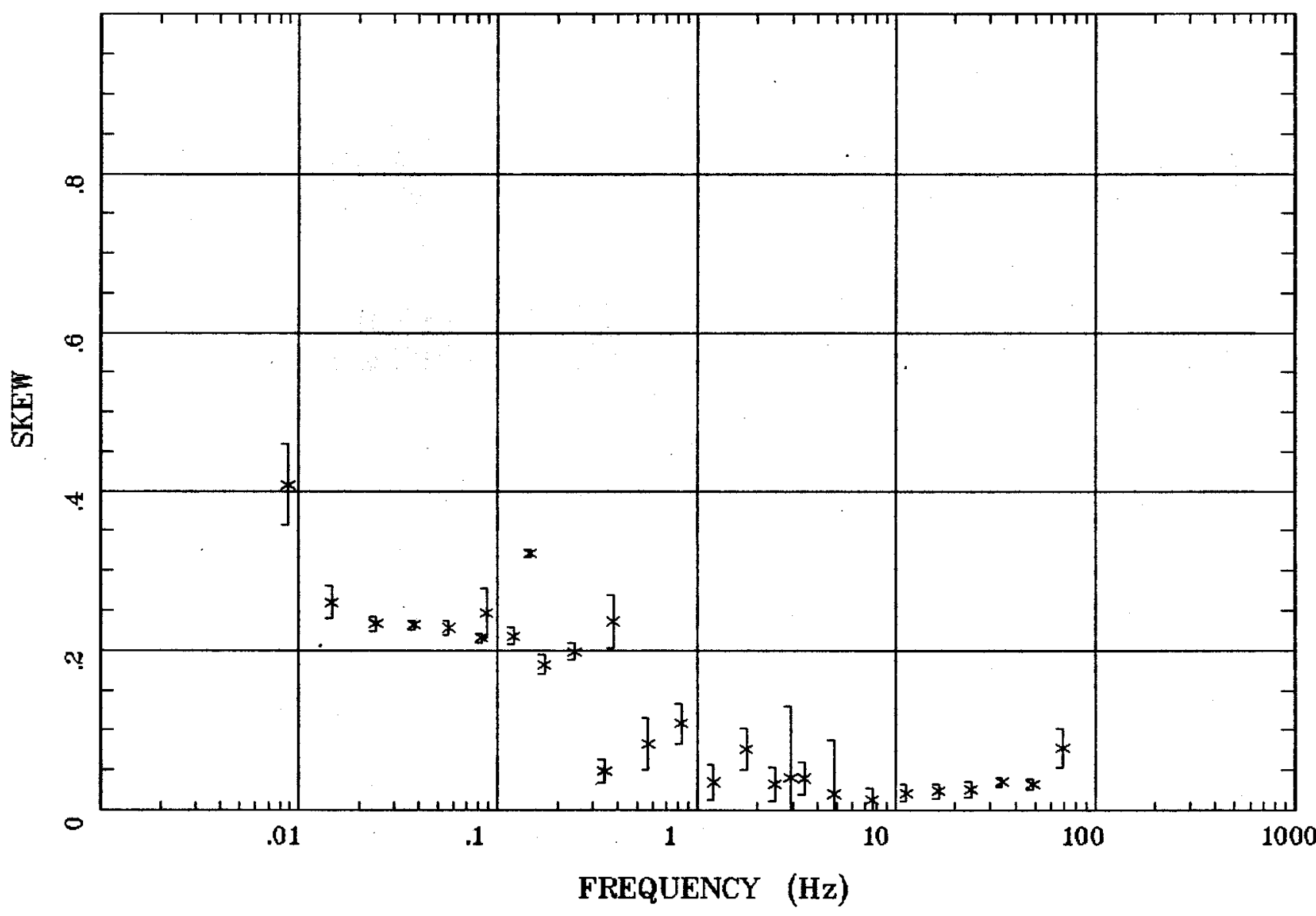

Client:

Remote: e-fld E $90 \mathrm{~m}$

Acquired: 14:4 Sep 17, 2003

Survey Co:USGS
Rotation:

Filename: ap21m.avg

Channels: Ch1 Ch2 Ch3 Ch4 Ch5 Ch6 Ch7

Plotted: 14:17 Sep 24, 2003

$<$ EMI - ElectroMagnetic Instruments 
E MULT Coh.

Ely, NV 100k

Station 21

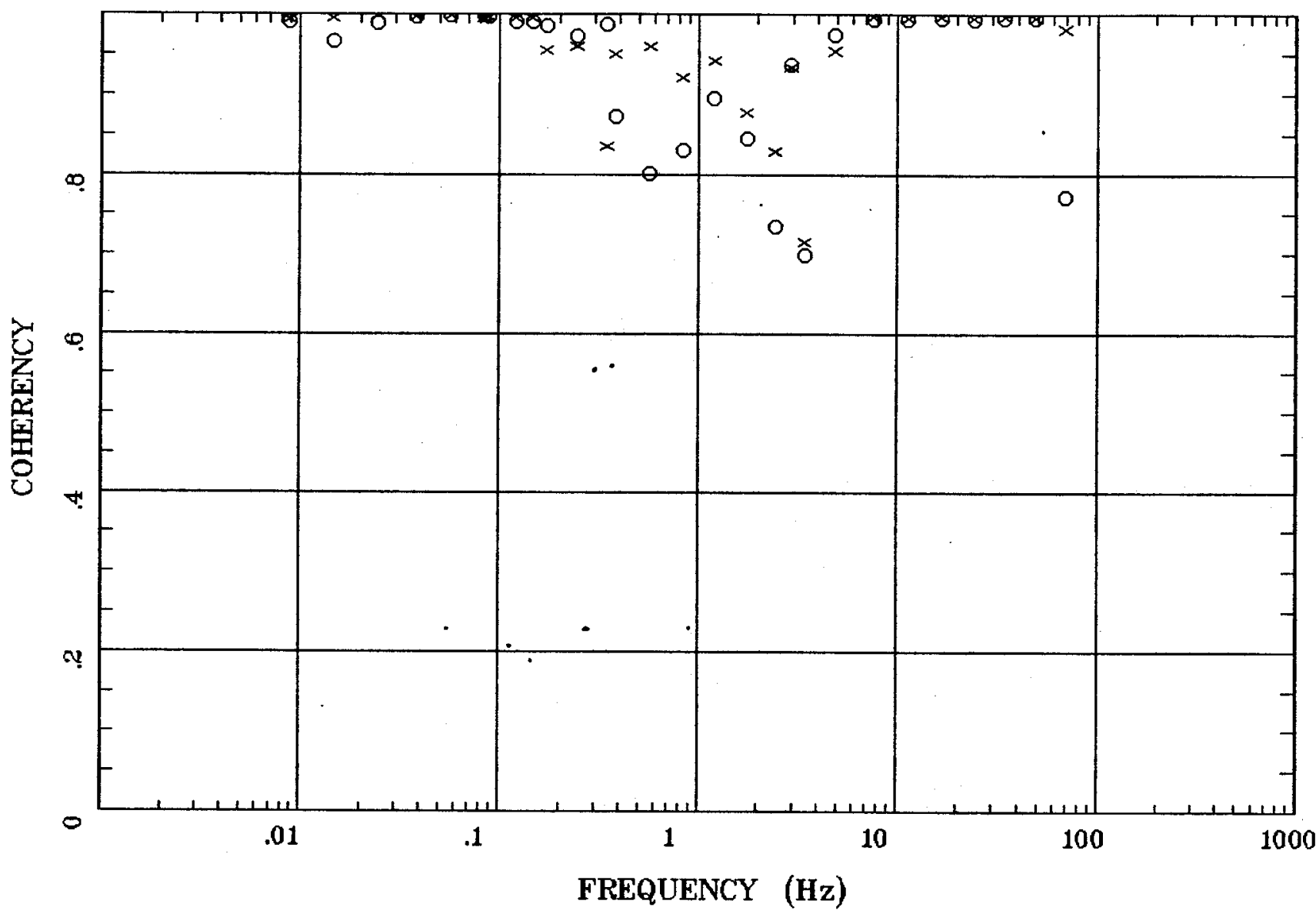

Client:

Remote: e-fld E $90 \mathrm{~m}$

Acquired: 14:4 Sep 17, 2003

Survey Co:USGS
Rotation:

Filename: ap21m.avg

Channels: Ch1 Ch2 Ch3 Ch4 Ch5 Ch6 Ch7 Plotted: 14:17 Sep 24, 2003

$<$ EMI - ElectroMagnetic Instruments 


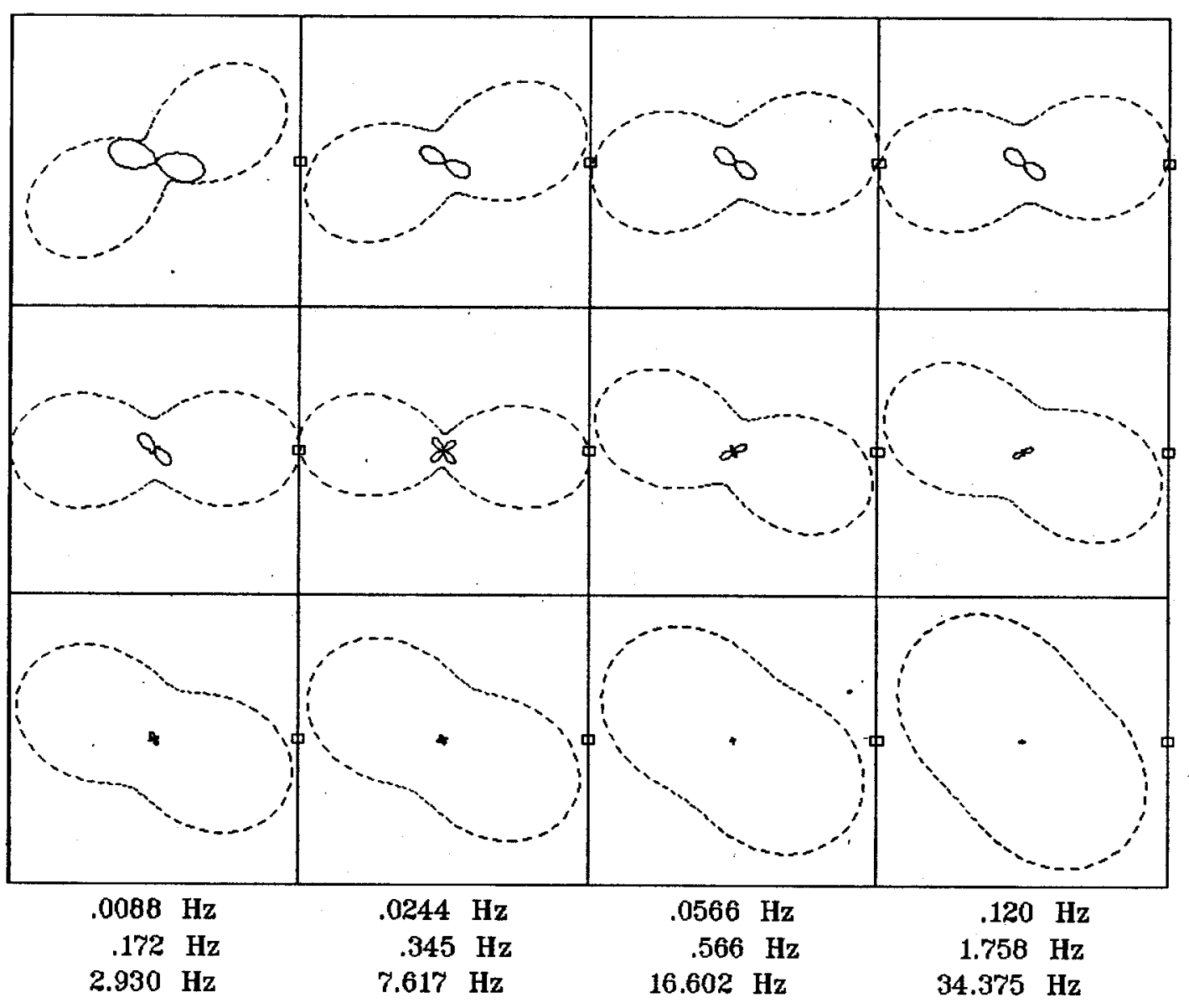

Client:

Remote: e-fld E $90 \mathrm{~m}$

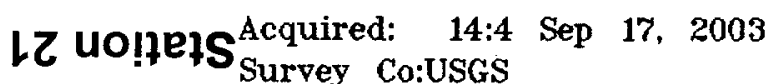

\section{Rotation:}

Filename: ap21m.avg

Channels: Ch1 Ch2 Ch3 Ch4 Ch5 Ch6 Ch7 Plotted: 14:17 Sep 24, 2003

$<$ EMI - ElectroMagnetic Instruments 


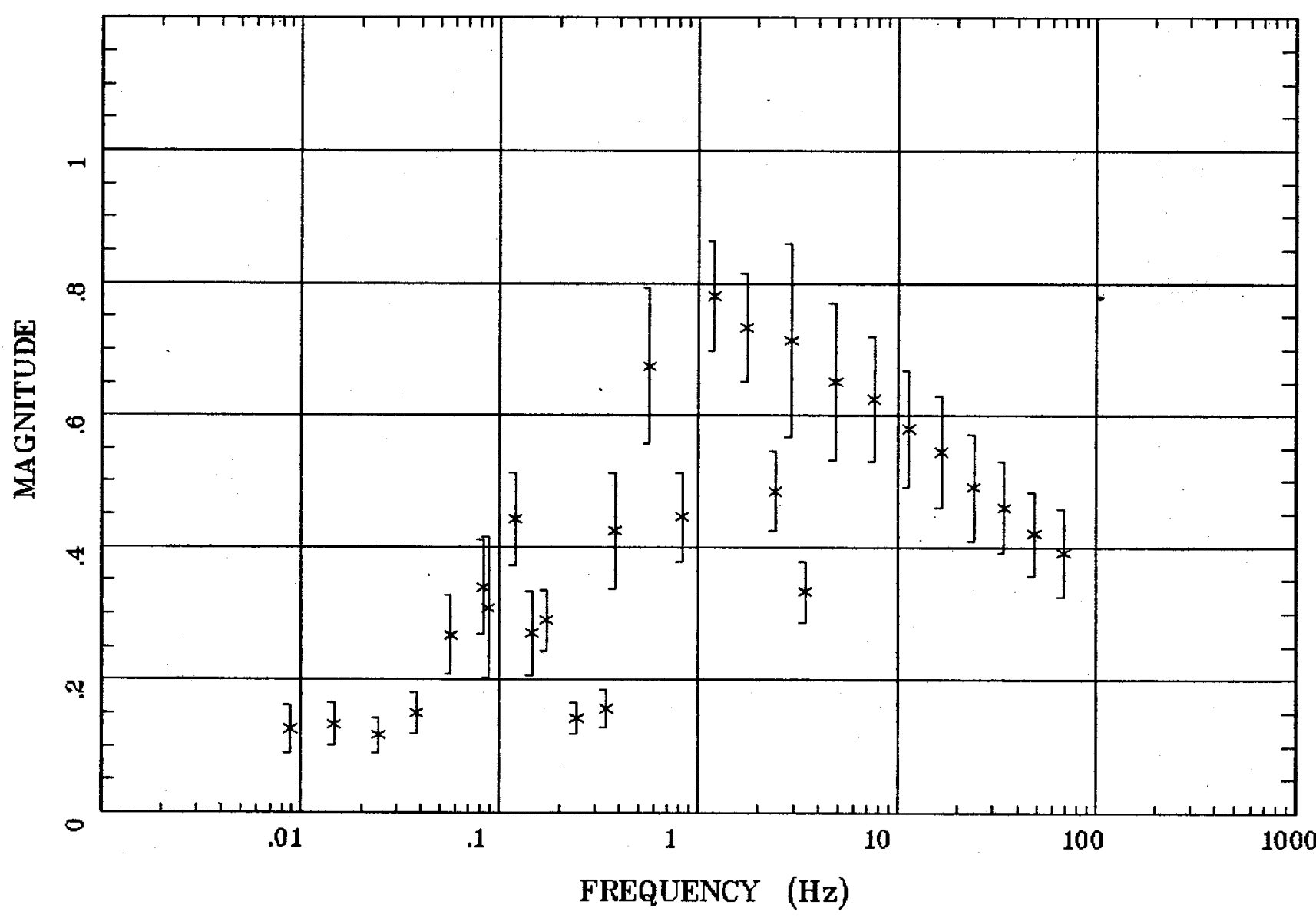

Client:

Remote: e-fld E $90 \mathrm{~m}$

Acquired: 14:4 Sep 17, 2003 Survey Co:USGS
Rotation:

Filename: ap21m.avg

Channels: Ch1 Ch2 Ch3 Ch4 Ch5 Ch6 Ch7

Plotted: 14:17 Sep 24, 2003

$<$ EMI - ElectroMagnetic Instruments 


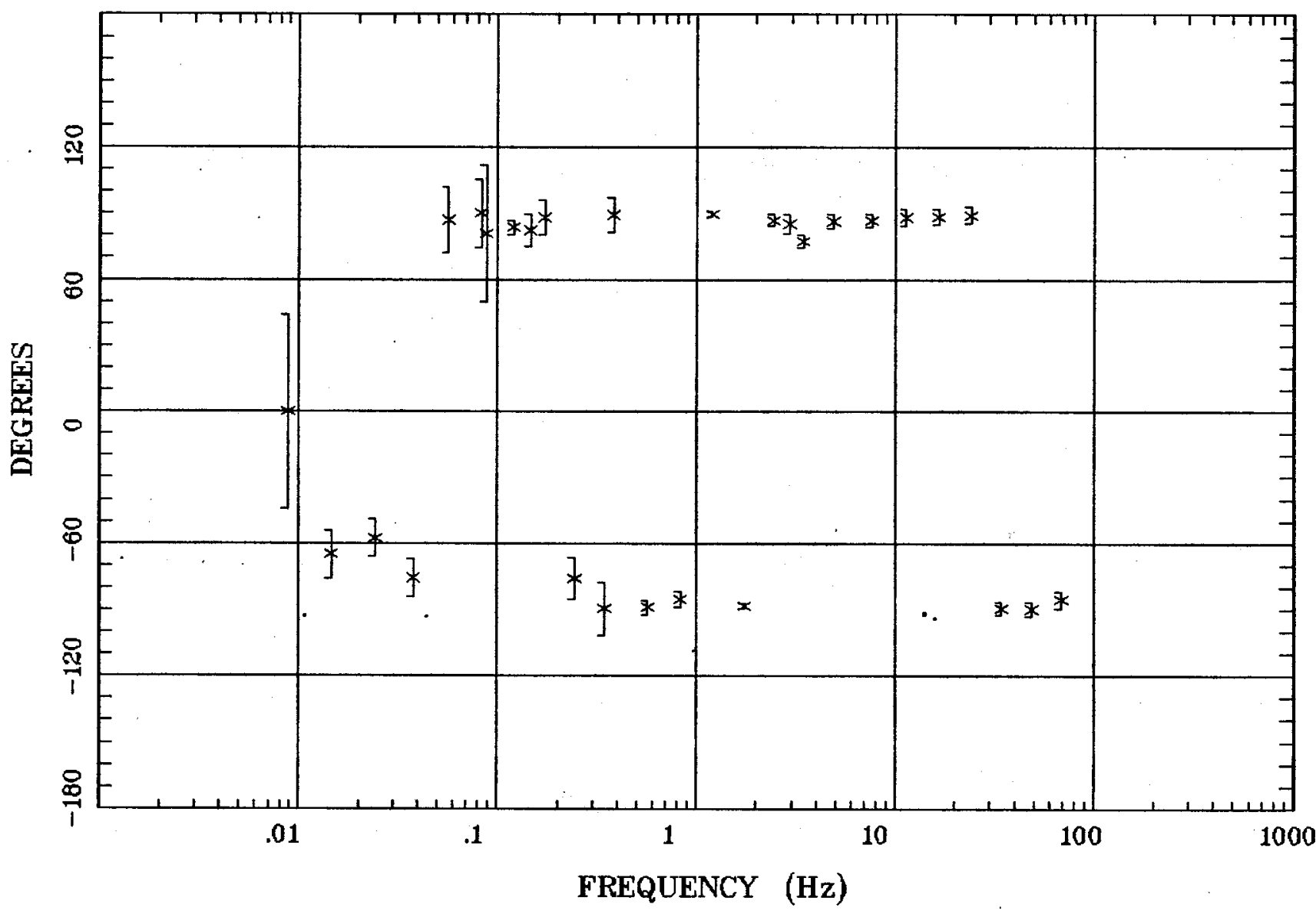

Client:

Remote: e-fld E $90 \mathrm{~m}$ Acquired: 14:4 Sep 17, 2003 Survey Co:USGS
Rotation:

Filename: ap21m.avg

Channels: Ch1 Ch2 Ch3 Ch4 Ch5 Ch6 Ch7

Platted: 14:17 Sep 24, 2003

< EMI - ElectroMagnetic Instruments 


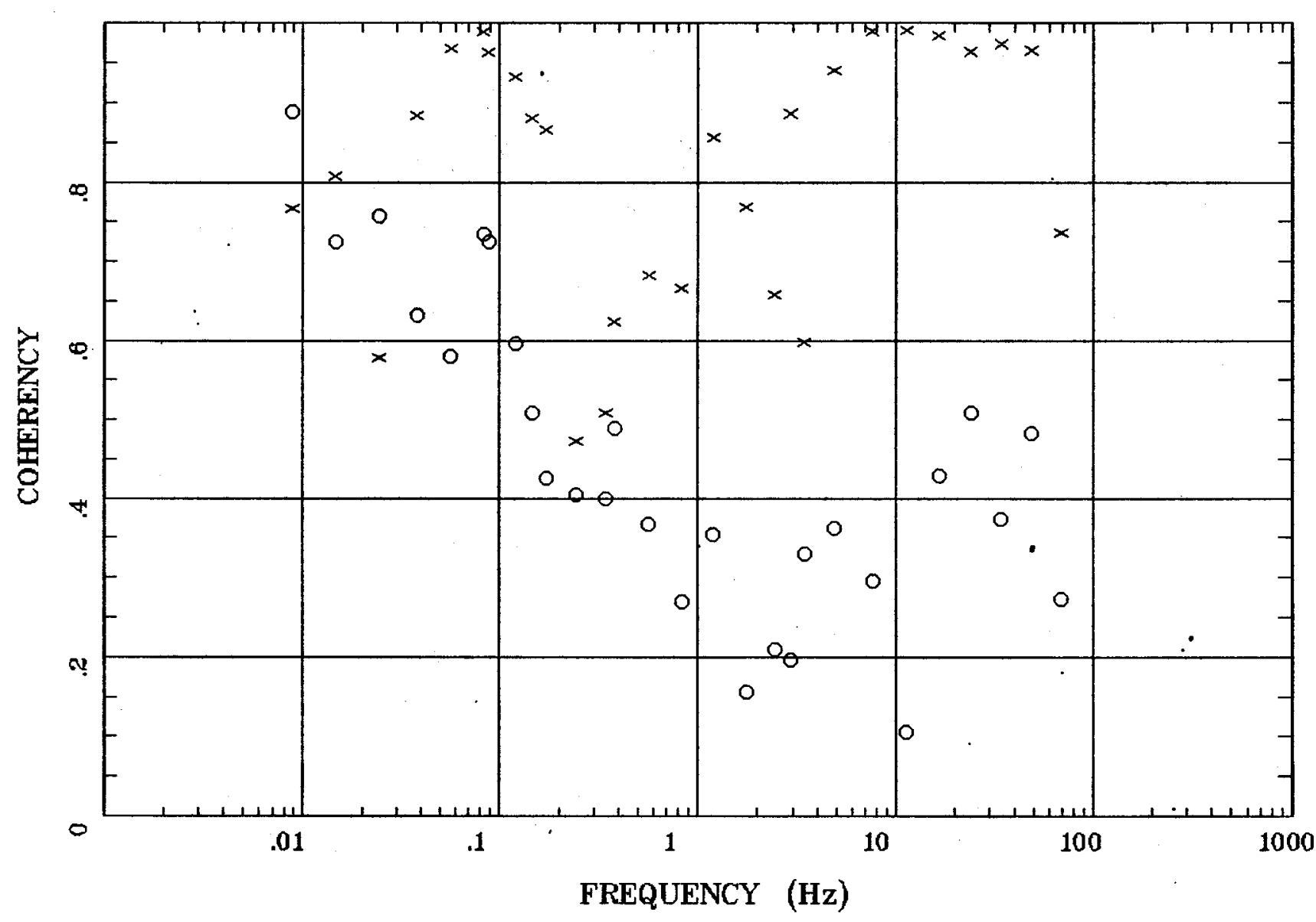

Client:

Remote: e-fld E $90 \mathrm{~m}$ Acquired: 14:4 Sep 17, 2003 Survey Co:USGS
Rotation:

Filename: ap21m.avg

Channels: Ch1 Ch2 Ch3 Ch4 Ch5 Ch6 Ch'7 Plotted: 14:17 Sep 24, 2003

$<$ EMI - ElectroMagnetic Instruments > 


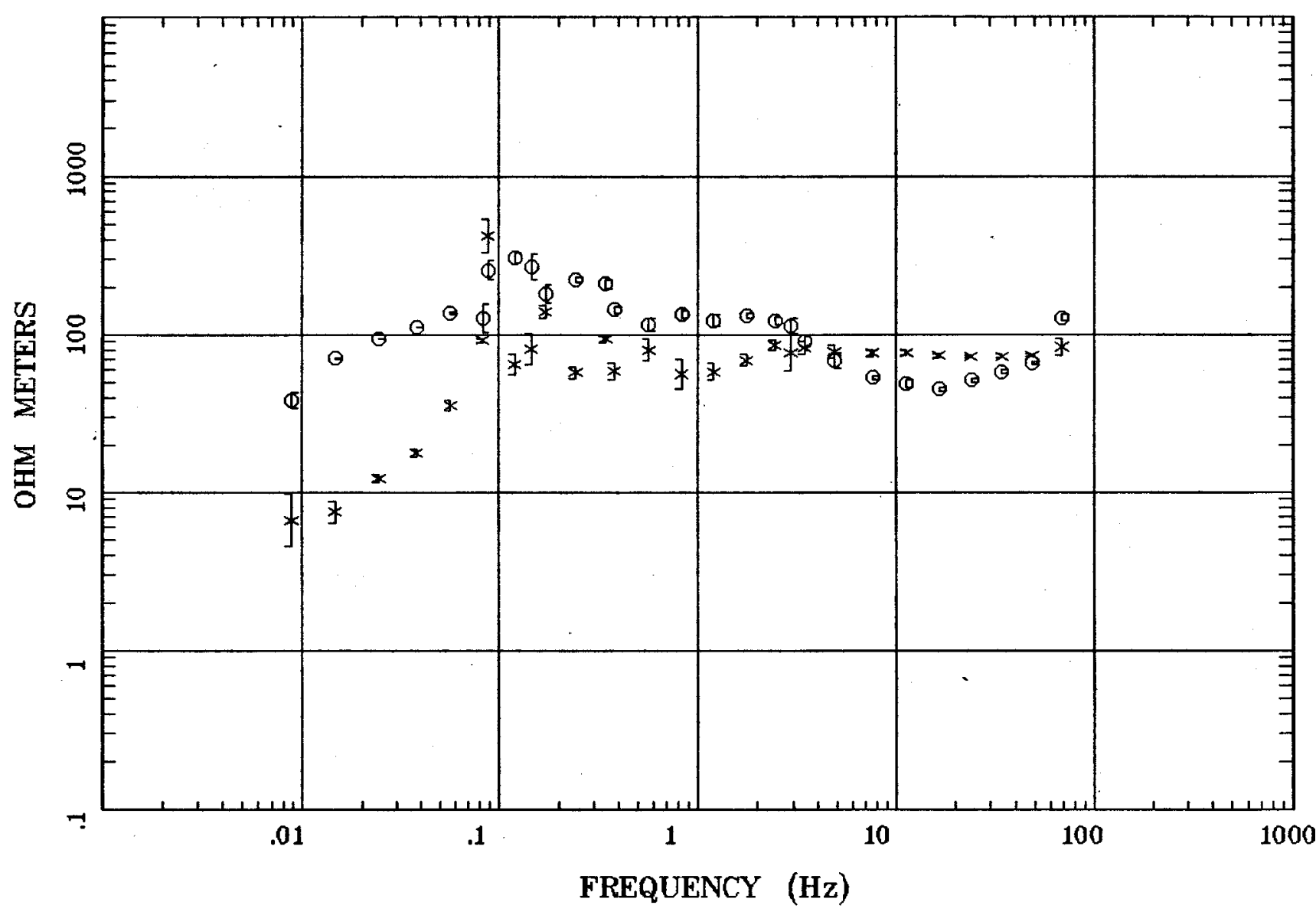

Client:

Remote: e-fld E $90 \mathrm{~m}$

Acquired: 13:2 Sep 15, 2003

Survey Co:USGS
Rotation:

Filename: ap22m.avg

Channels: Ch1 Ch2 Ch3 Ch4 Ch5 ch6 Ch7

Plotted: 11:24 Sep 24, 2003

< EMI - ElectroMagnetic Instruments 


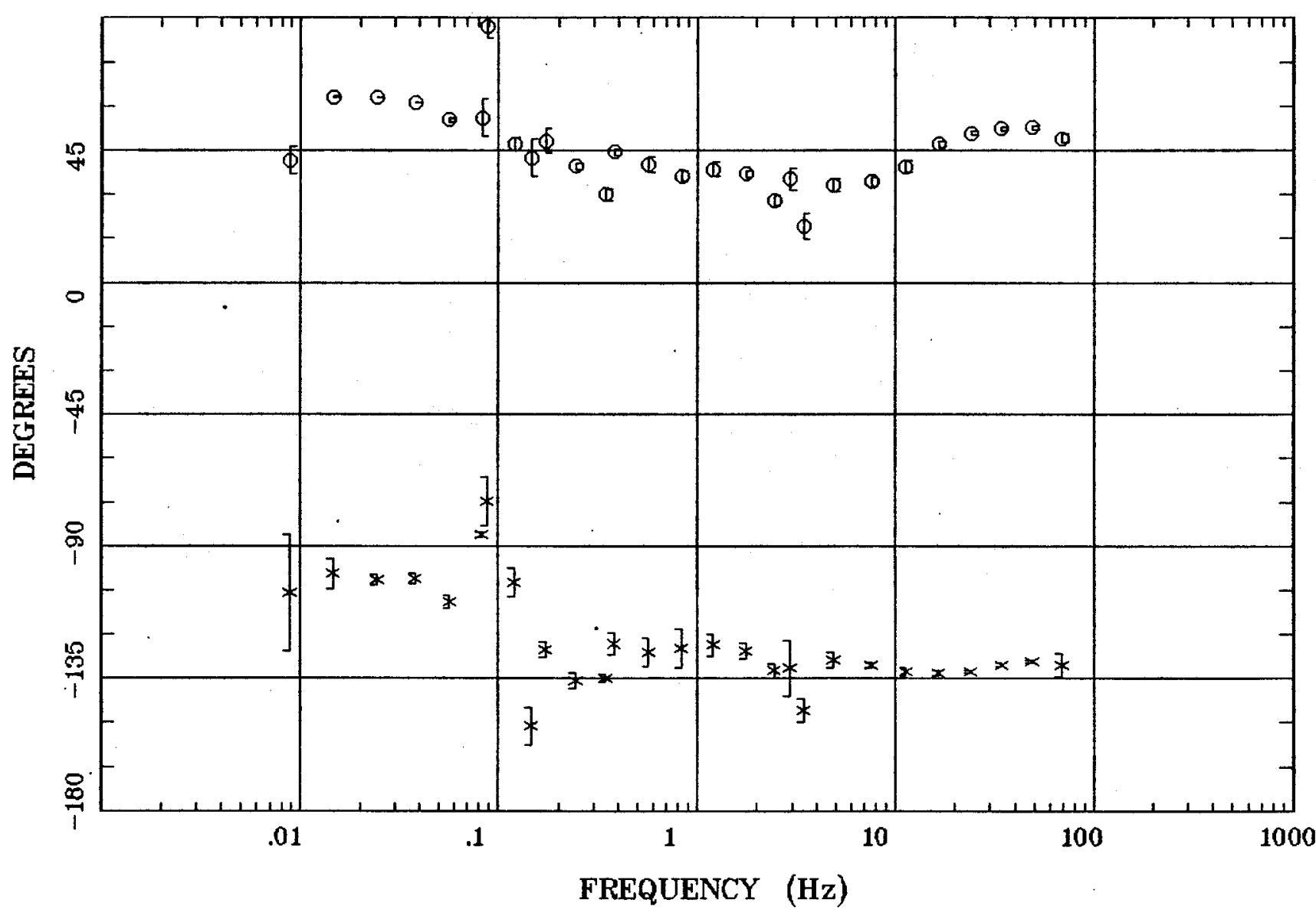

Client:

Remote: e-fld E $90 \mathrm{~m}$ Acquired: 13:2 Sep 15, 2003

Survey Co:USGS
Rotation:

Filename: ap22m.avg

Channels: Ch1 Ch2 Ch3 Ch4 Ch5 Ch6 Ch7

Plotted: 11:24 Sep 24, 2003

< EMI - ElectroMagnetic Instruments 


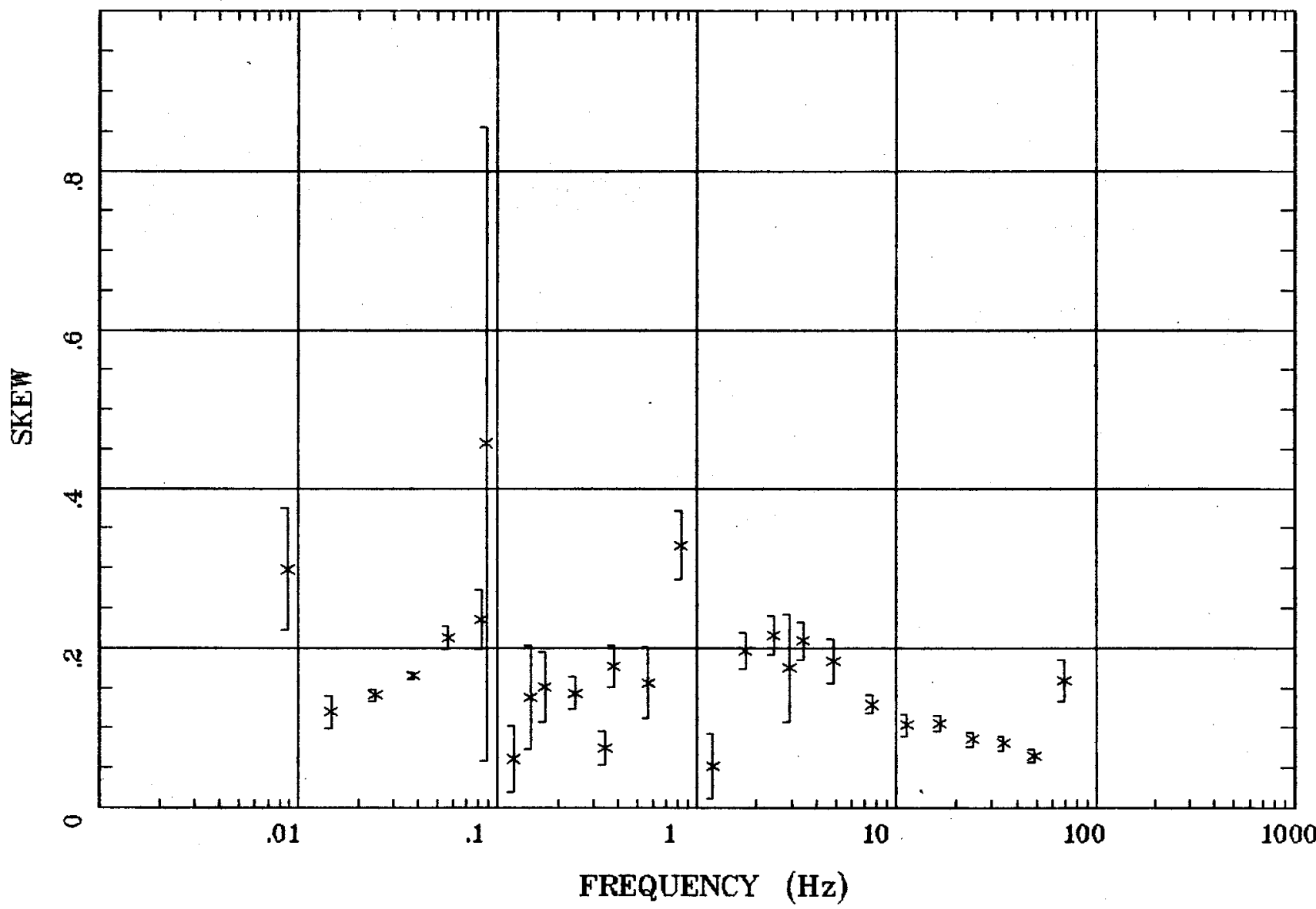

Client:

Remote: e-fld E $90 \mathrm{~m}$ Acquired: 13:2 Sep 15, 2003 Survey Co:USGS
Rotation:

Filename: ap22m.avg

Channels: Ch1 Ch2 Ch3 Ch4 Ch5 Ch6 Ch7 Plotted: 11:24 Sep 24, 2003

< EMI - ElectroMagnetic Instruments > 
E MULT Coh.

Ely, NV 100k

Station 22

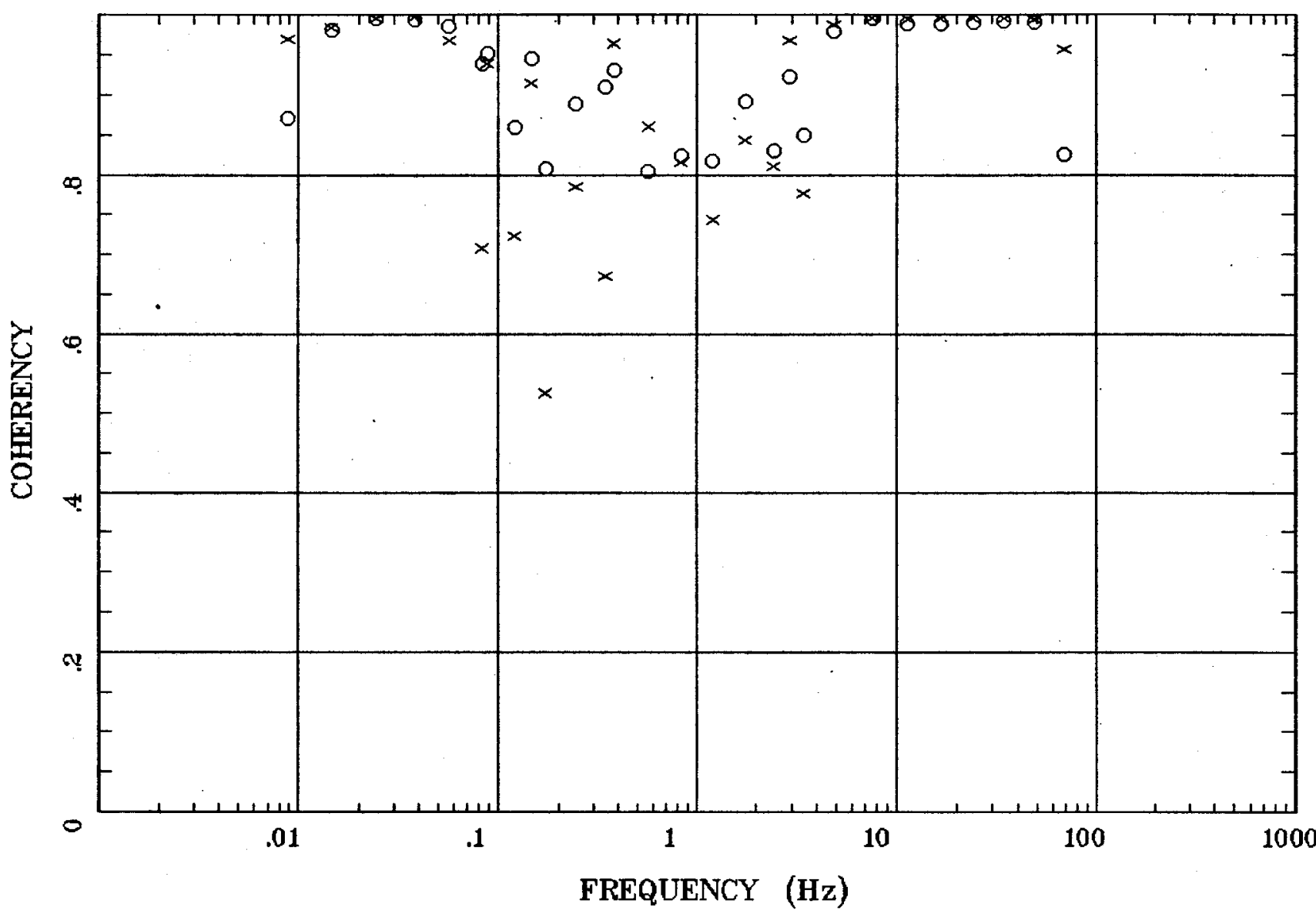

Client:

Remote: e-fld E $90 \mathrm{~m}$

Acquired: 13:2 Sep 15, 2003

Survey Co:USGS
Rotation:

Filename: ap22m.avg

Channels: Ch1 Ch2 Ch3 Ch4 Ch5 Ch6 Ch7

Plotted: 11:24 Sep 24, 2003

< EMI - ElectroMagnetic Instruments 


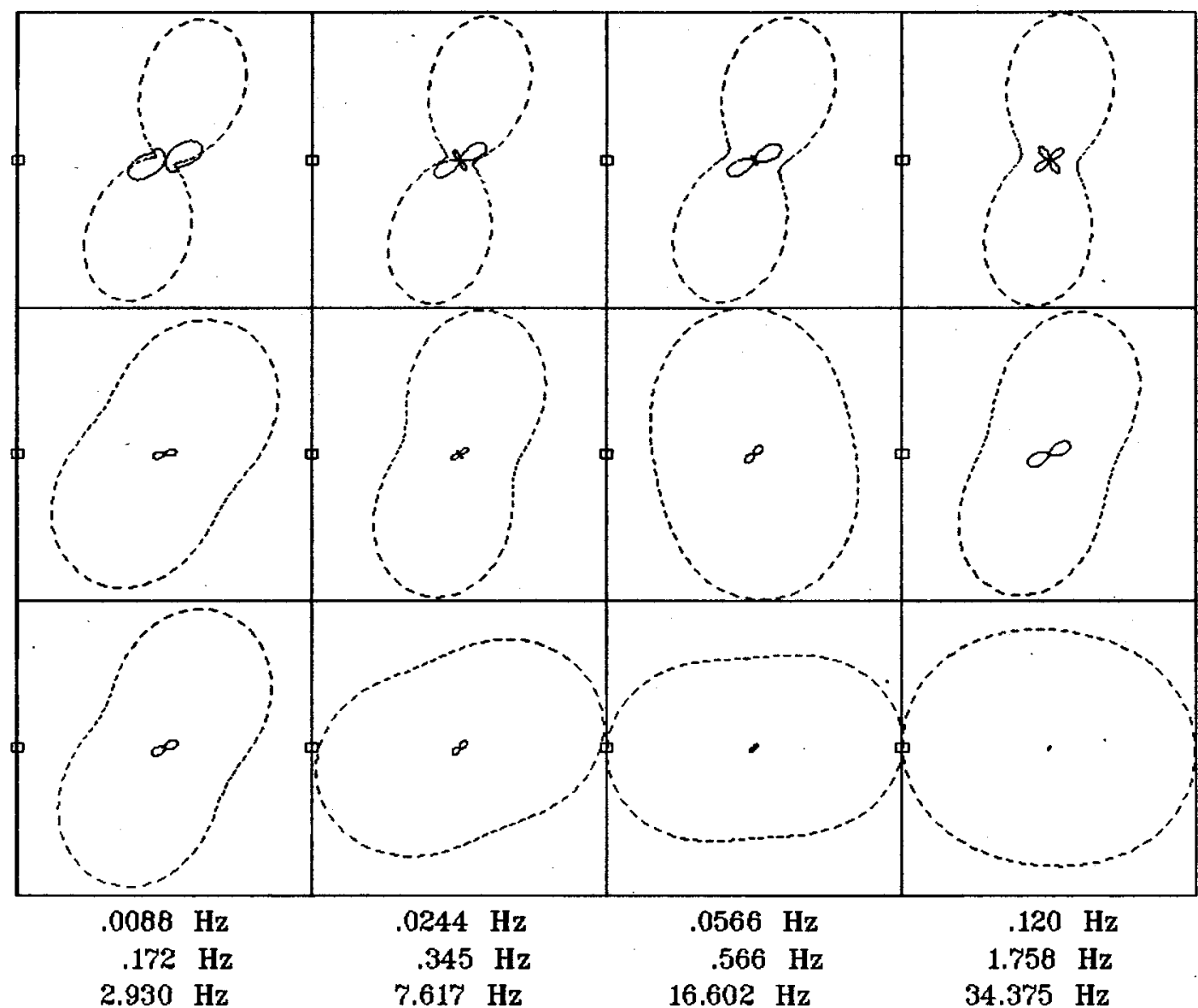

Client:

Remote: e-fld E $90 \mathrm{~m}$ Acquired: 13:2 Sep 15, 2003 Survey Co:USGS

\section{Rotation:}

Filename: ap22m.avg

Channels: Ch1 Ch2 Ch3 Ch4 Ch5 Ch6 Ch7

Plotted: 11:24 Sep 24, 2003

< EMI - ElectroMagnetic Instruments > 


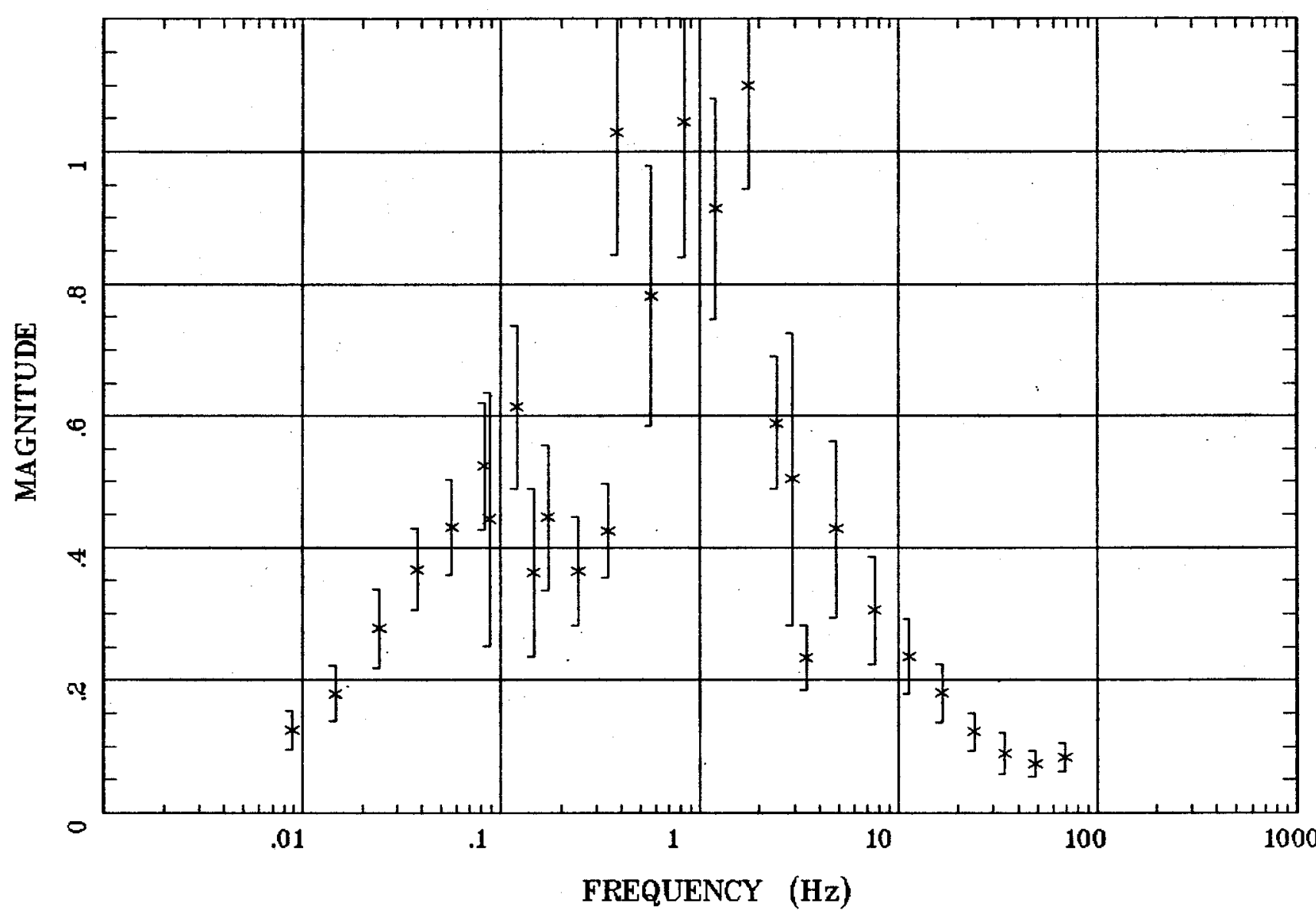

Client:

Remote: e-fld E $90 \mathrm{~m}$

Acquired: 13:2 Sep 15, 2003

Survey Co:USGS
Rotation:

Filename: ap22m.avg

Channels: Ch1 Ch2 Ch3 Ch4 Ch5 Ch6 Ch7

Plotted: 11:24 Sep 24, 2003

$<$ EMI - ElectroMagnetic Instruments 


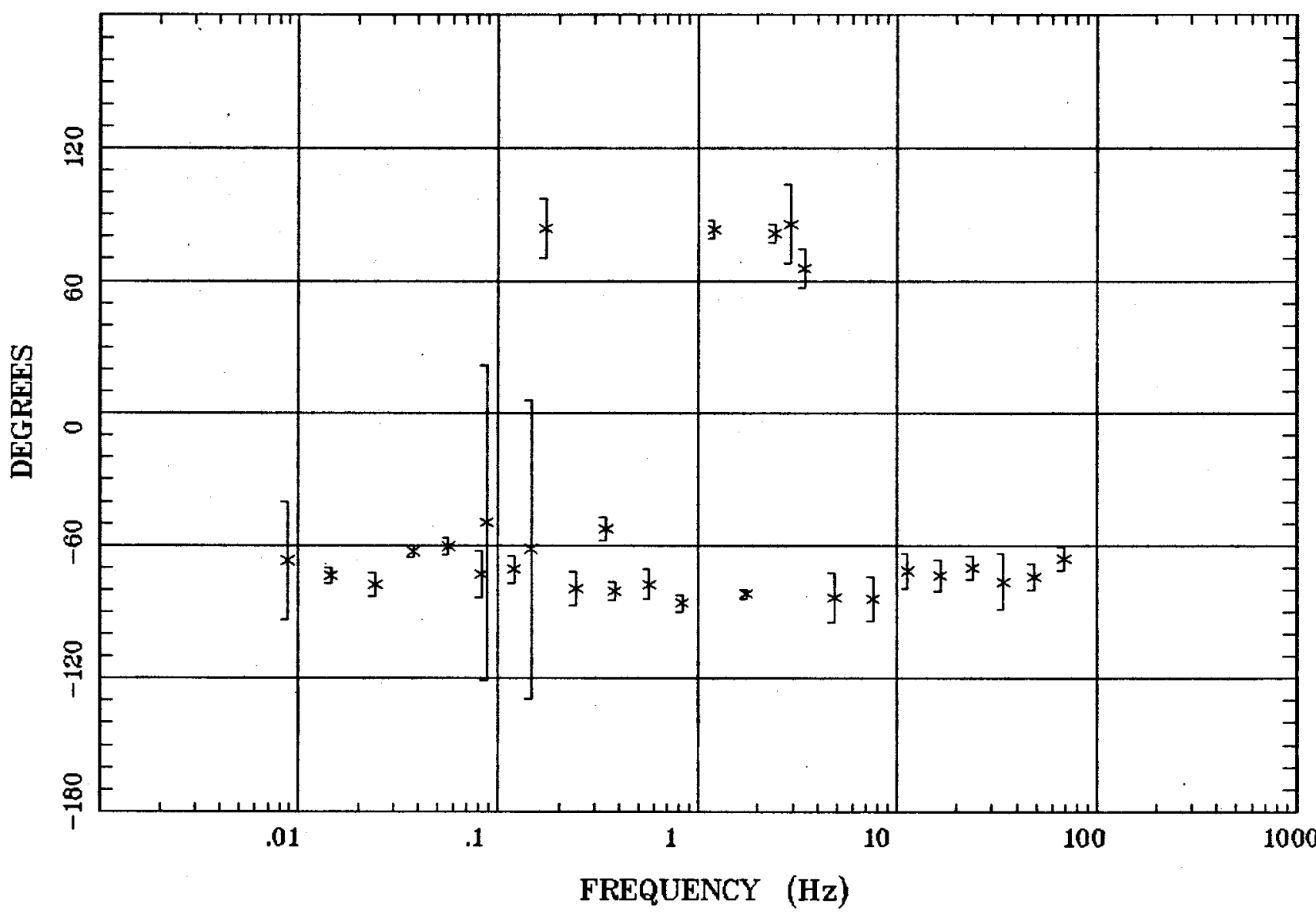

Client:

Remote: e-fld E $90 \mathrm{~m}$

Acquired: 13:2 Sep 15, 2003

Survey Co:USGS
Rotation:

Filename: ap22m.avg

Channels: Ch1 Ch2 Ch3 Ch4 Ch5 Ch6 Ch7

Plotted: 11:24 Sep 24, 2003

< EMI - ElectroMagnetic Instruments 


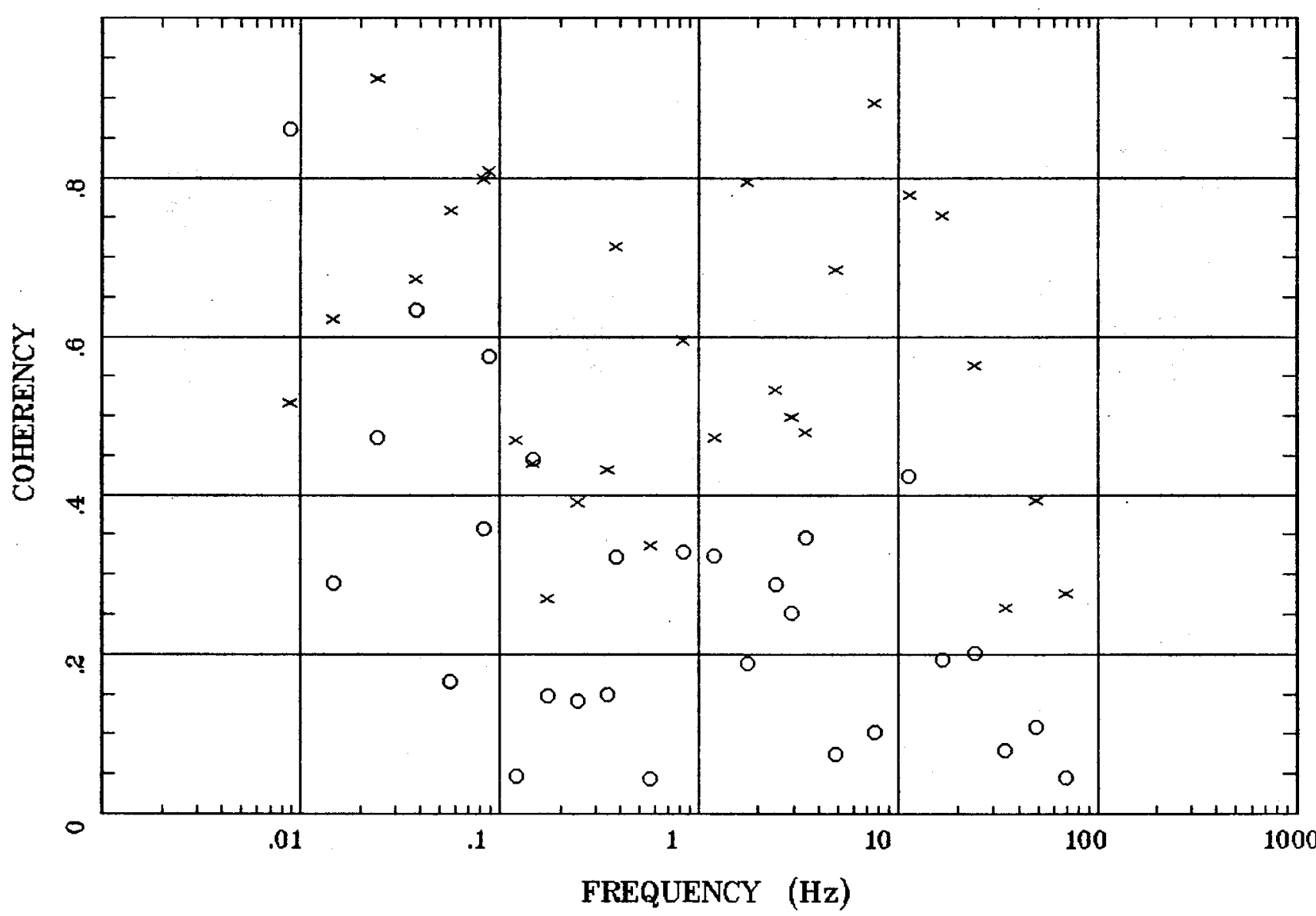

Client:

Remote: e-fld E $90 \mathrm{~m}$

Acquired: 13:2 Sep 15, 2003

Survey Co:USGS
Rotation:

Filename: ap22m.avg

Channels: Ch1 Ch2 Ch3 Ch4 Ch5 Ch6 Ch7

Plotted: 11:24 Sep 24, 2003

< EMI - ElectroMagnetic Instruments 


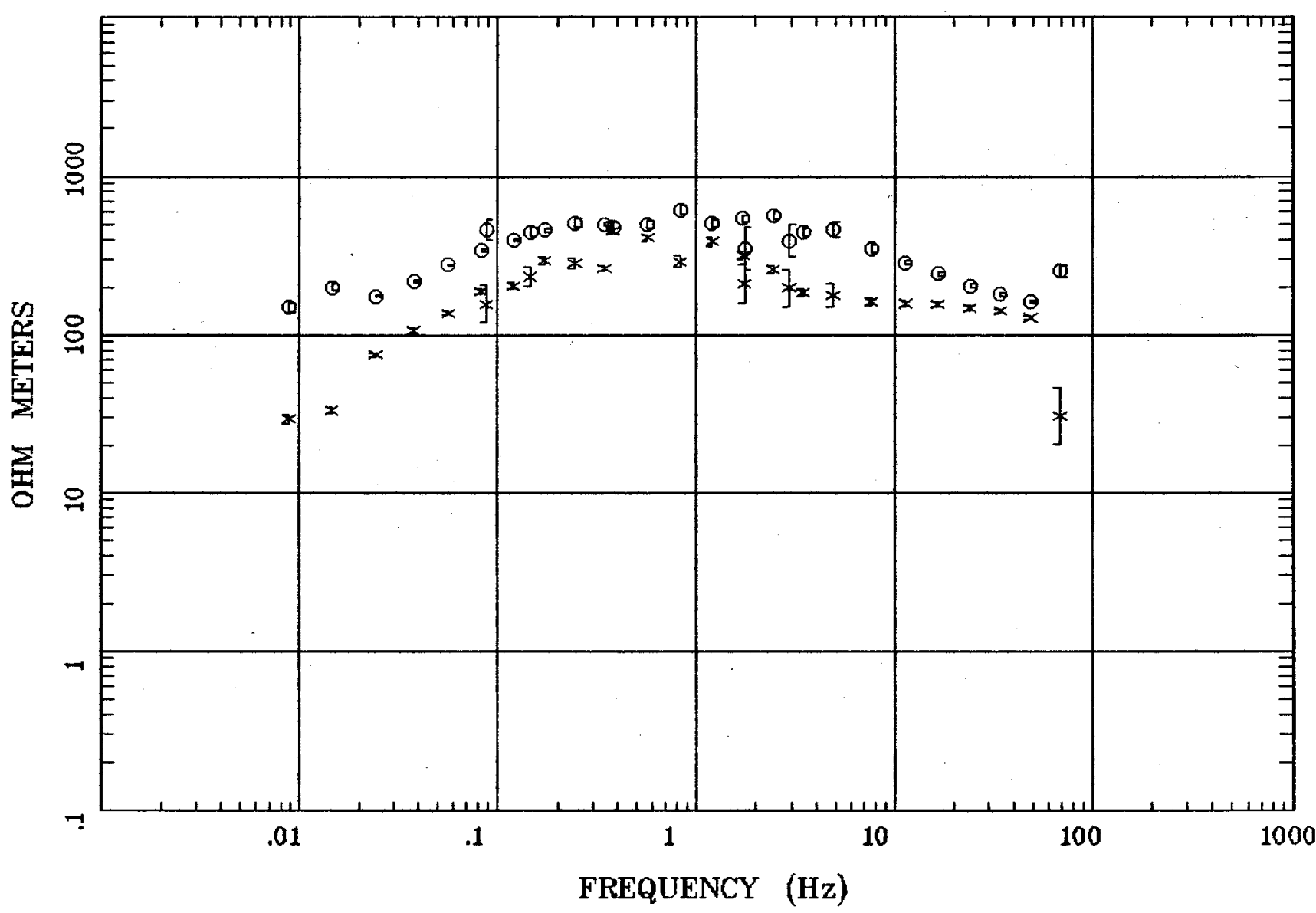

Client:

Remote: e-fld E $90 \mathrm{~m}$

Acquired: 17:1 Sep 15, 2003 Survey Co:USGS
Rotation:

Filename: ap23mall.avg

Channels: Ch1 Ch2 Ch3 Ch4 Ch5 Ch6 Ch7

Plotted: 11:25 Sep 24, 2003

< EMI - ElectroMagnetic Instruments 


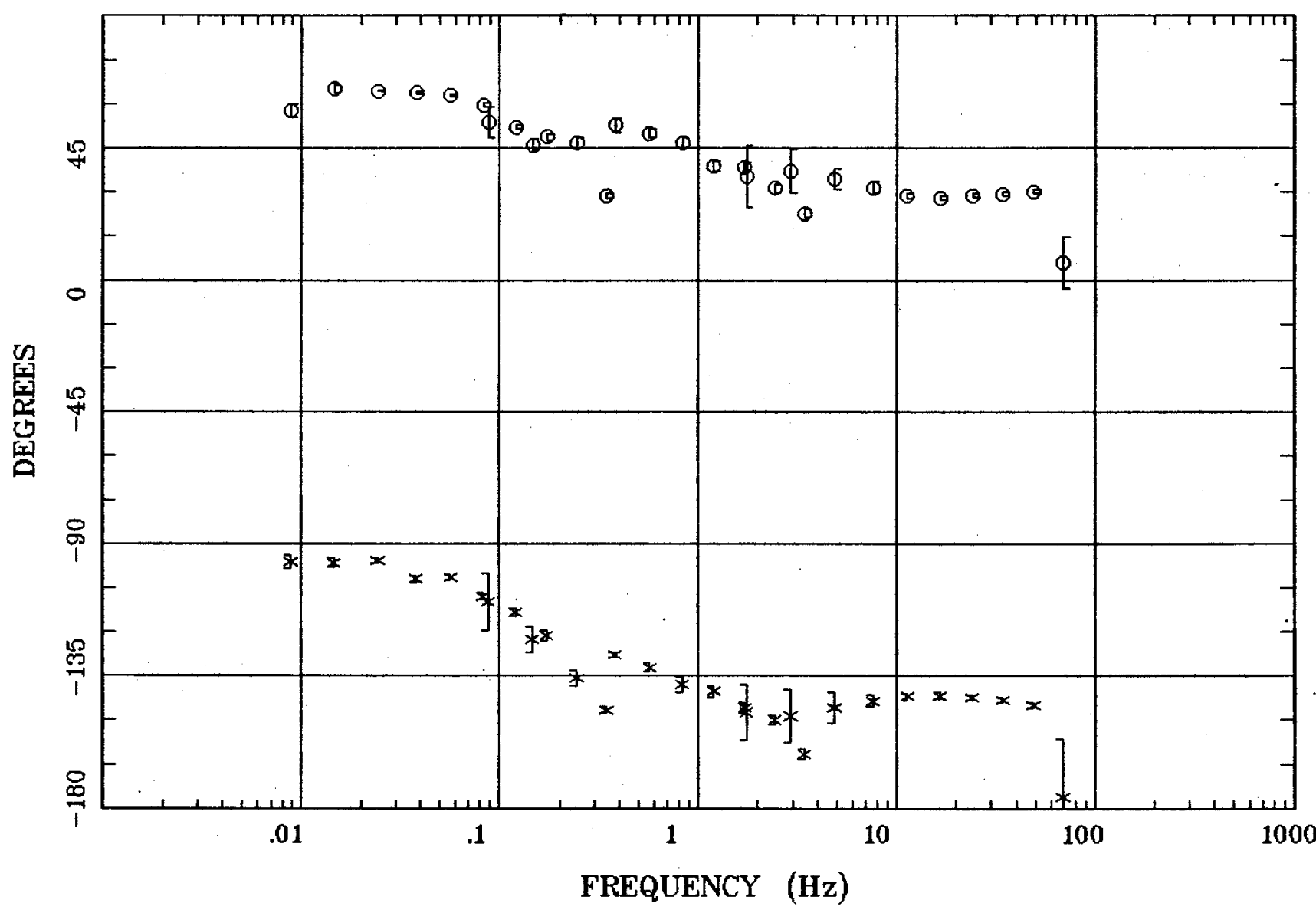

Client:

Remote: e-fld E $90 \mathrm{~m}$

Acquired: $17: 1 \operatorname{Sep} 15,2003$ Survey Co:USGS
Ratation:

Filename: ap23mall.avg Channels: Ch1 Ch2 Ch3 Ch4 Ch5 Ch6 Ch7 Plotted: 11:25 Sep 24, 2003

< EMI - ElectroMagnetic Instruments 


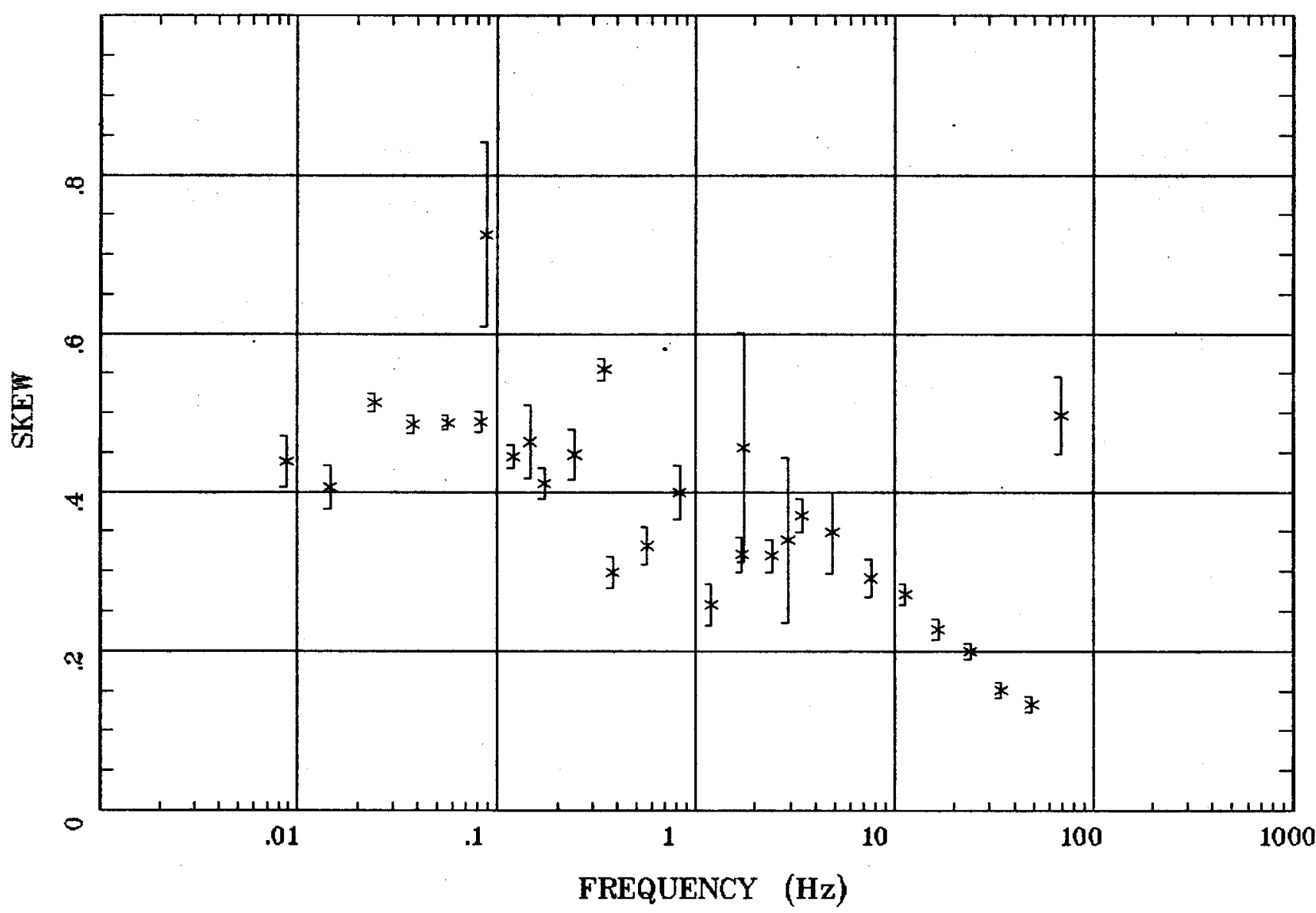

Client:

Remote: e-fld E 90 m

Acquired: 17:1 Sep 15, 2003 Survey Co:USGS
Rotation:

Filename: ap23mall.avg

Channels: Ch1 Ch2 Ch3 Ch4 Ch5 Ch6 Chr

Plotted: 11:25 Sep 24, 2003

$<$ EMI - ElectroMagnetic Instruments 
E MULT Coh.

Ely, NV 100k

Station 23

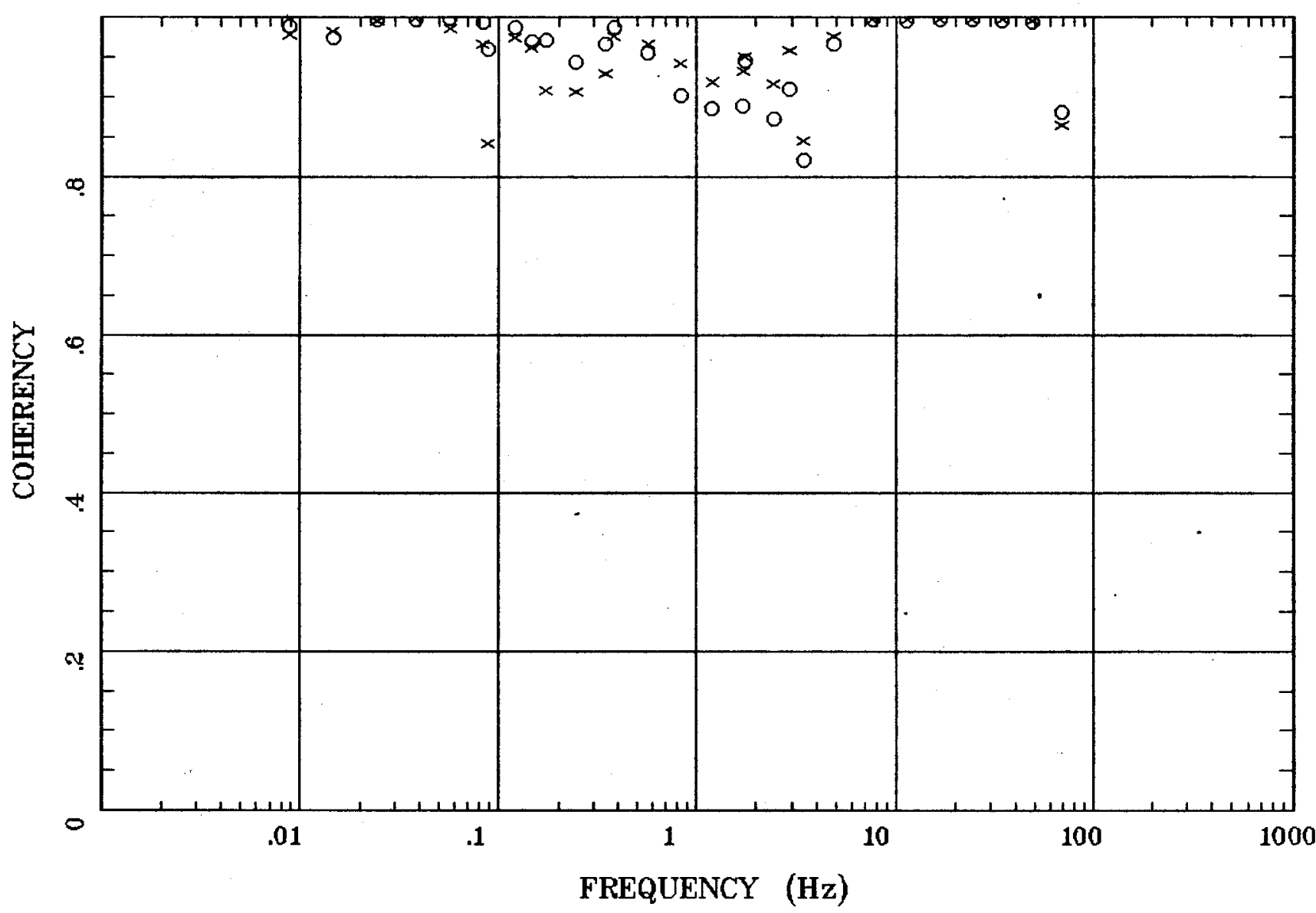

Client:

Remote: e-fld E $90 \mathrm{~m}$

Acquired: 17:1 Sep 15, 2003 Survey Co:USGS
Rotation:

Filename: ap23mall.avg

Channels: Ch1 Ch2 Ch3 ch4 Ch5 Ch6 Ch7

Plotted: 11:25 Sep 24, 2003

$<$ EMI - ElectroMagnetic Instruments 


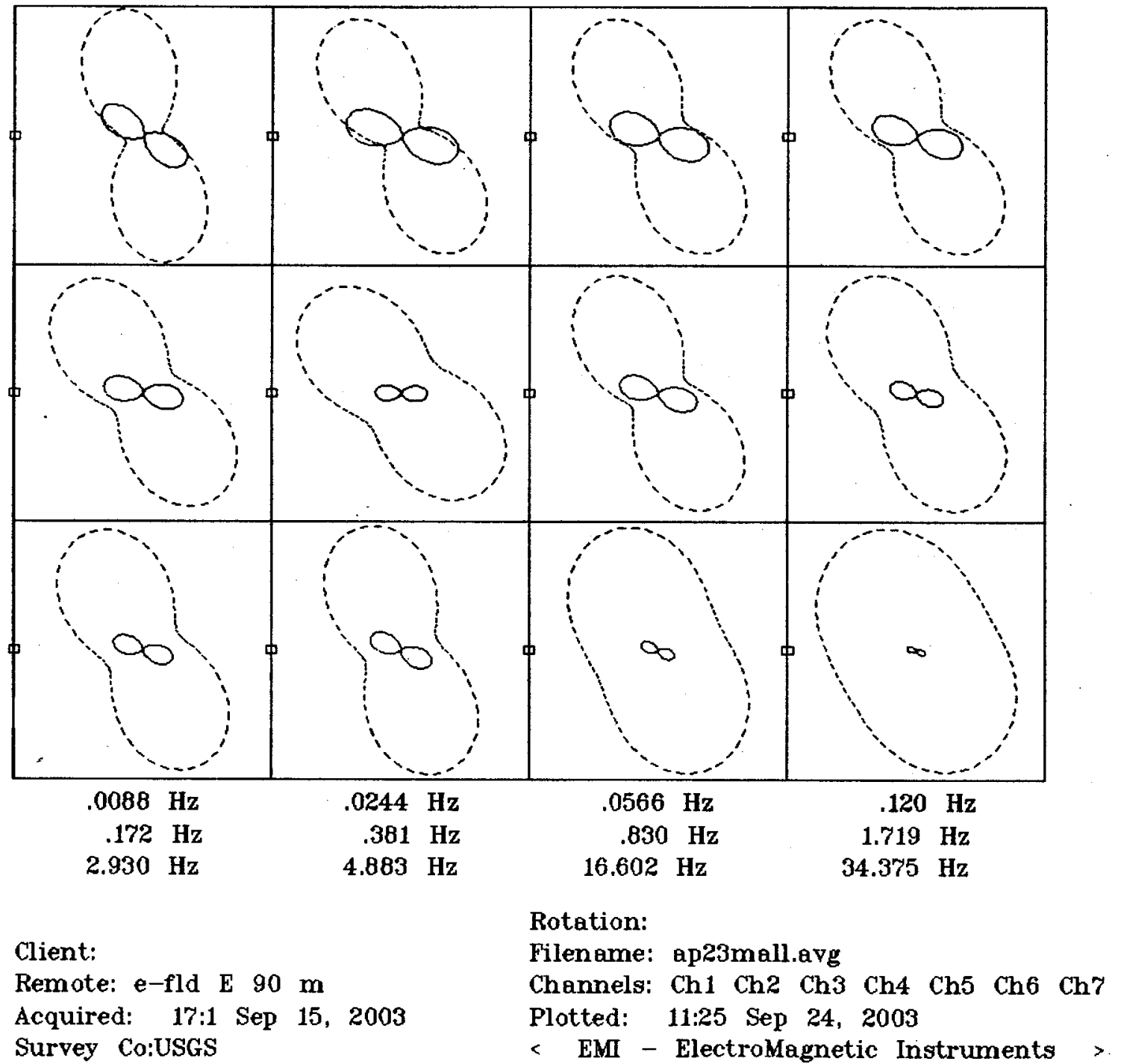




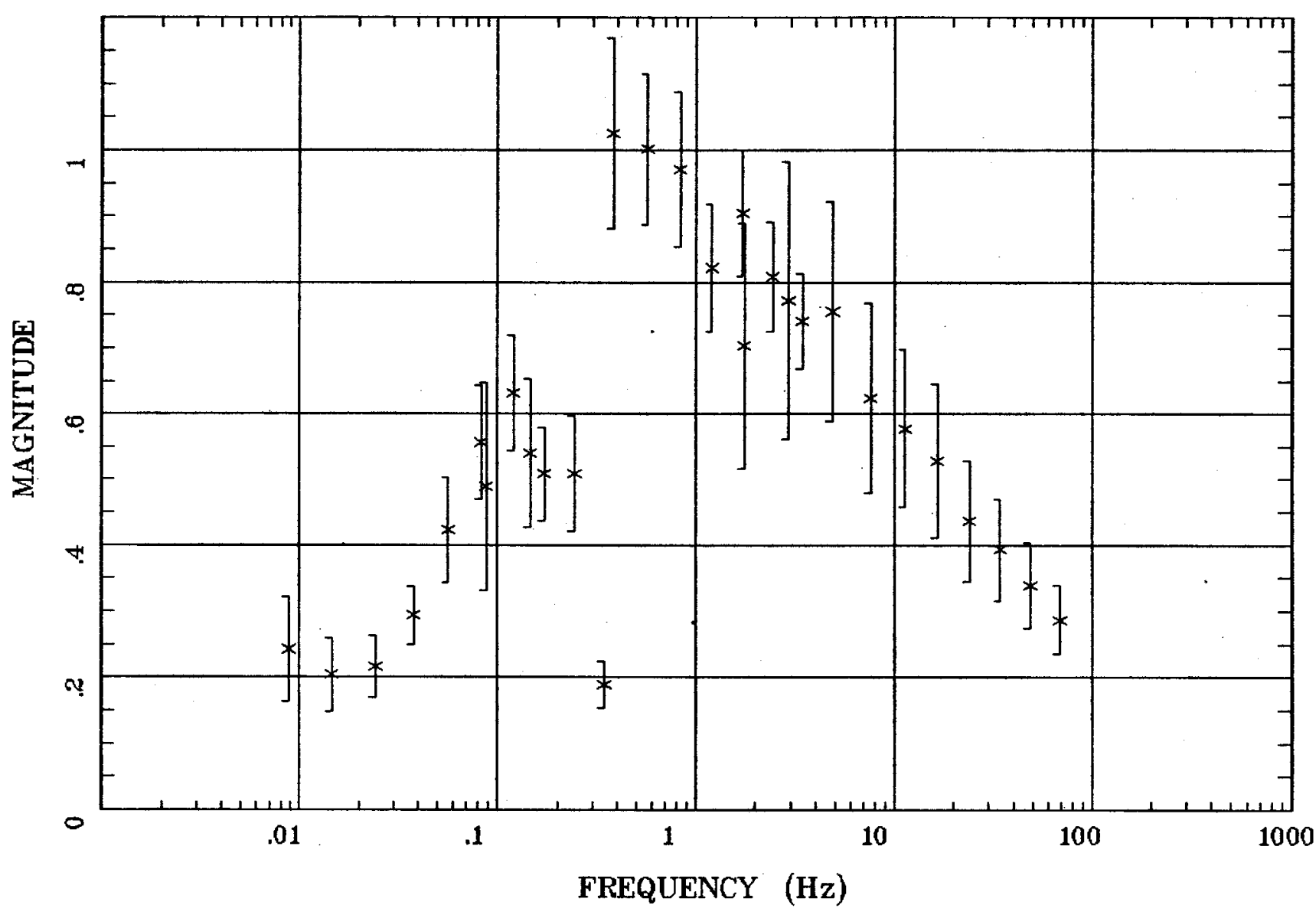

Client:

Remote: e-fld E $90 \mathrm{~m}$

Acquired: 17:1 Sep 15, 2003 Survey Co:USGS
Rotation:

Filename: ap23mall.avg

Channels: Ch1 Ch2 Ch3 Ch4 Ch5 Ch6 Ch7 Plotted: 11:25 Sep 24, 2003

< EMI - ElectroMagnetic Instruments > 


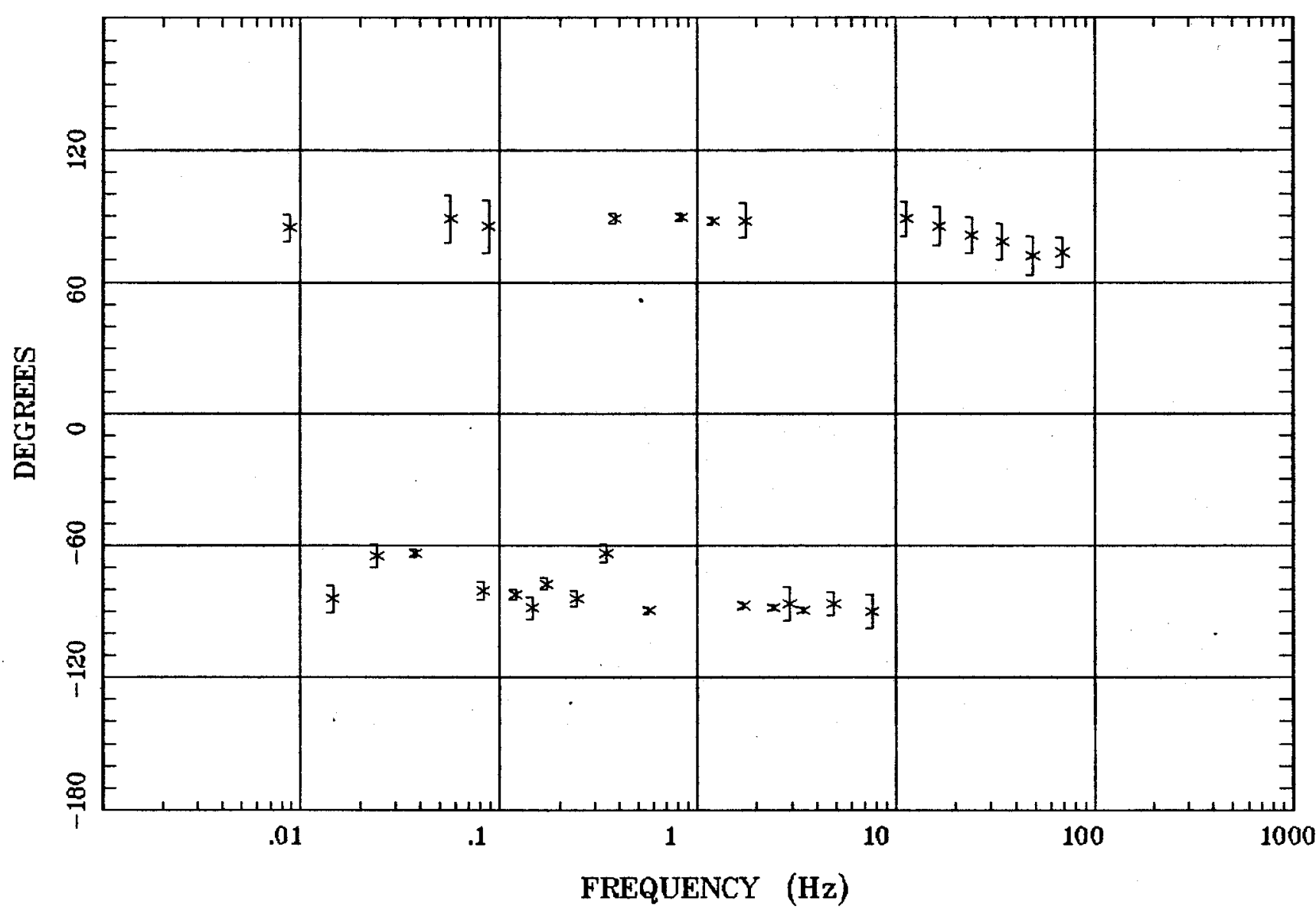

Client:

Remote: e-fld E $90 \mathrm{~m}$

Acquired: 17:1 Sep 15, 2003 Survey Co:USGS
Rotation:

Filename: ap23mall.avg

Channels: Ch1 Ch2 Ch3 Ch4 Ch5 Ch6 Chr

Plotted: 11:25 Sep 24, 2003

$<$ EMI - ElectroMagnetic Instruments 


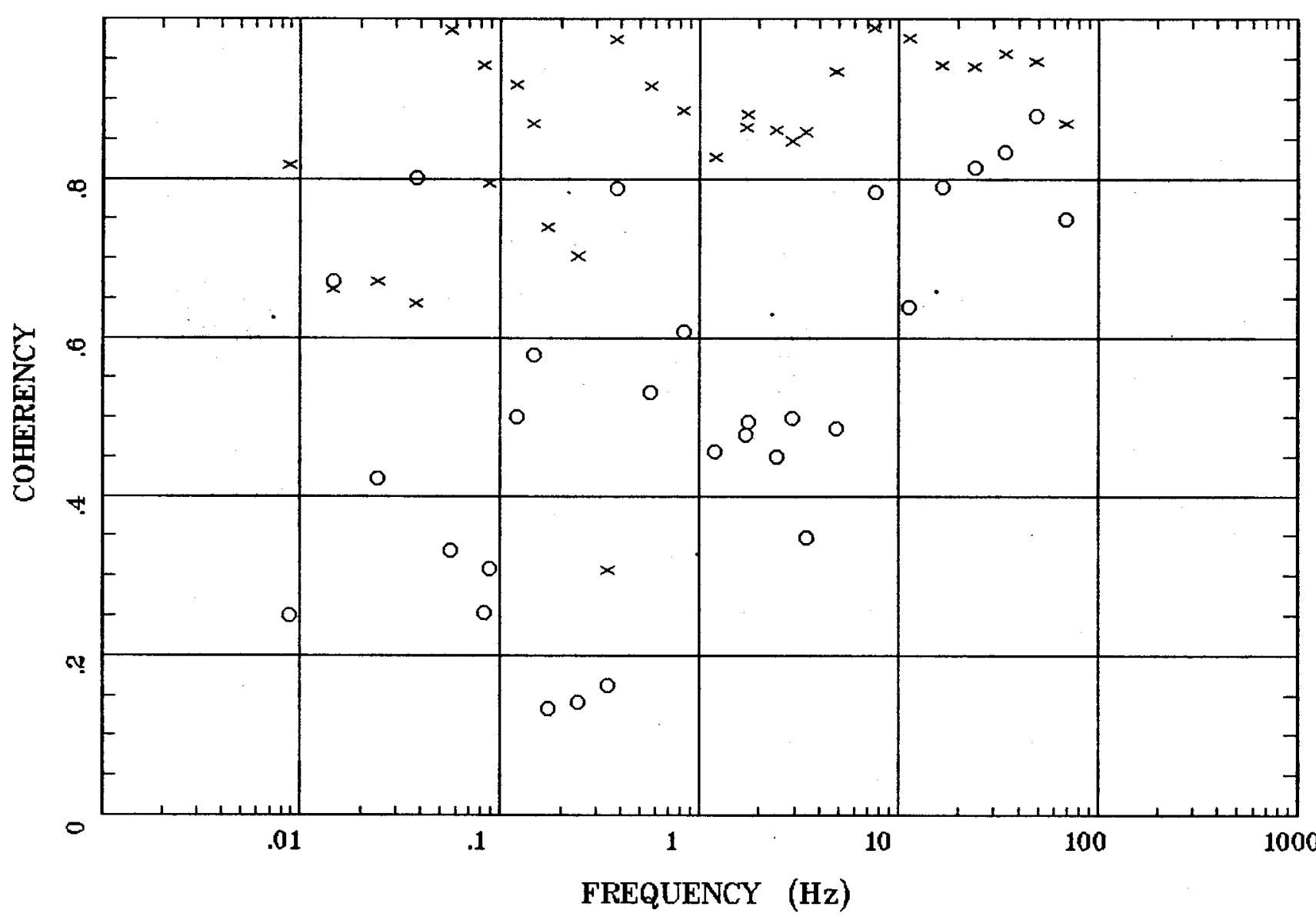

Client:

Remote: e-fld E $90 \mathrm{~m}$

Acquired: 17:1 Sep 15, 2003

Survey Co:USGS
Rotation:

Filename: ap23mall.avg

Channels: Ch1 Ch2 Ch3 Ch4 Ch5 Ch6 Ch7

Plotted: 11:25 Sep 24, 2003

\& EMI - ElectroMagnetic Instruments 


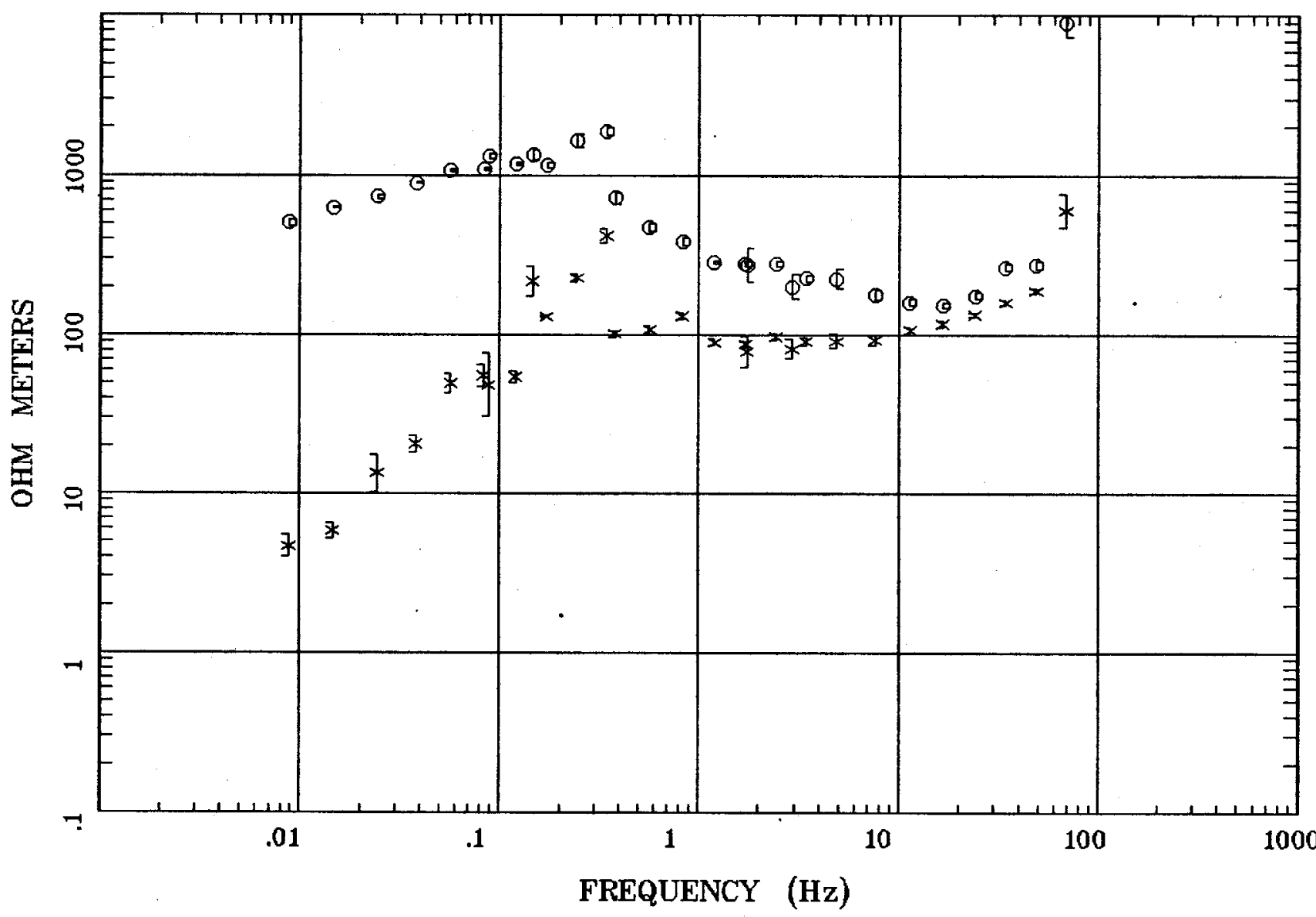

Client:

Remote: e-fld N $90 \mathrm{~m}$

Acquired: 10:2 Sep 18, 2003 Survey Co:USGS
Rotation:

Filename: ap24mall.avg

Channels: Ch1 Ch2 Ch3 Ch4 Ch5 Ch6 Ch7

Plotted: 11:25 Sep 24, 2003

< EMI - ElectroMagnetic Instruments 


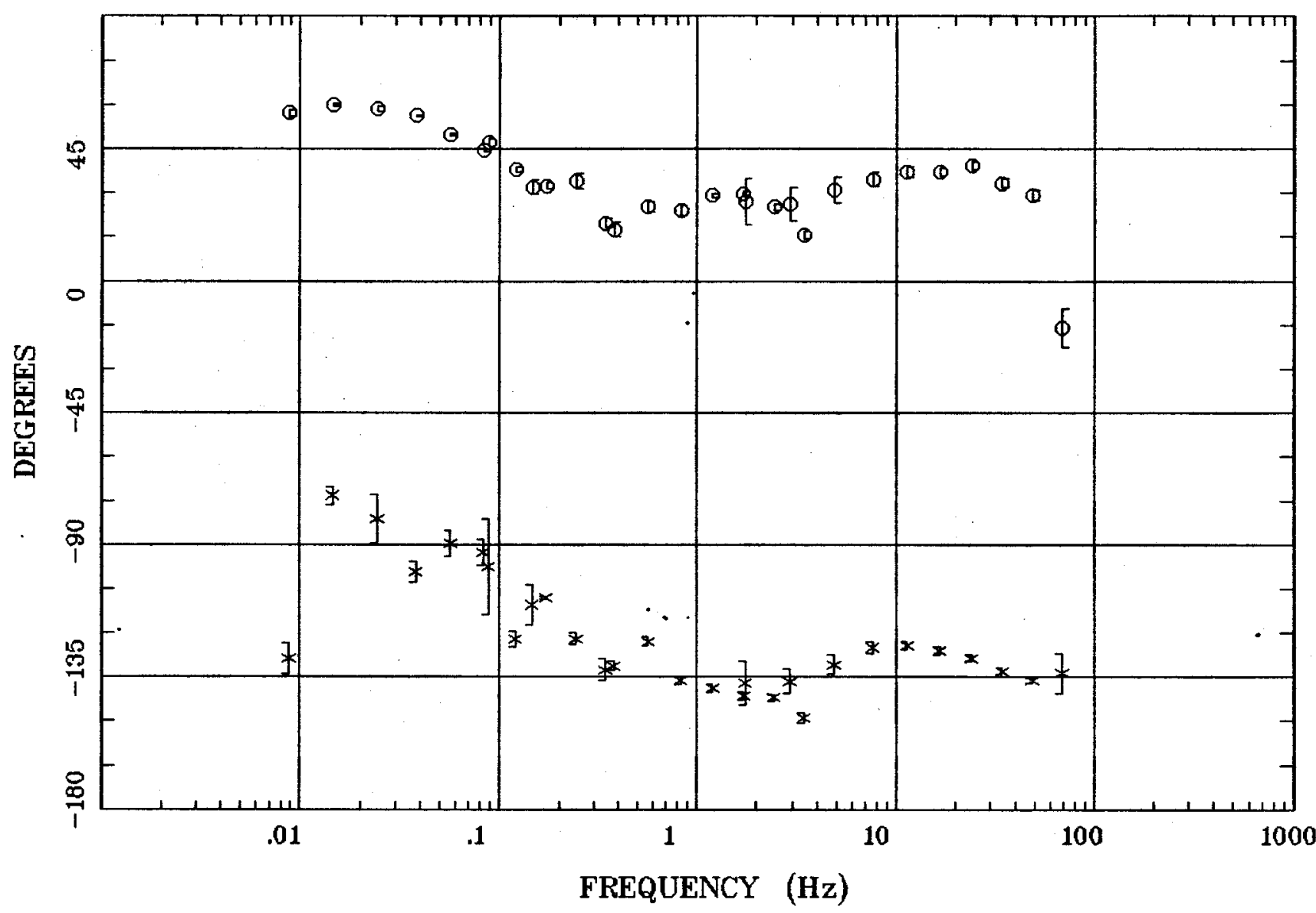

Client:

Remate: e-fld N $90 \mathrm{~m}$ Acquired: 10:2 Sep 18, 2003 Survey Co:USGS
Rotation:

Filename: ap24mall.avg

Channels: Ch1 Ch2 Ch3 Ch4 Ch5 Ch6 Ch7 Plotted: 11:25 Sep 24, 2003

$<$ EMI - ElectroMagnetic Instruments 


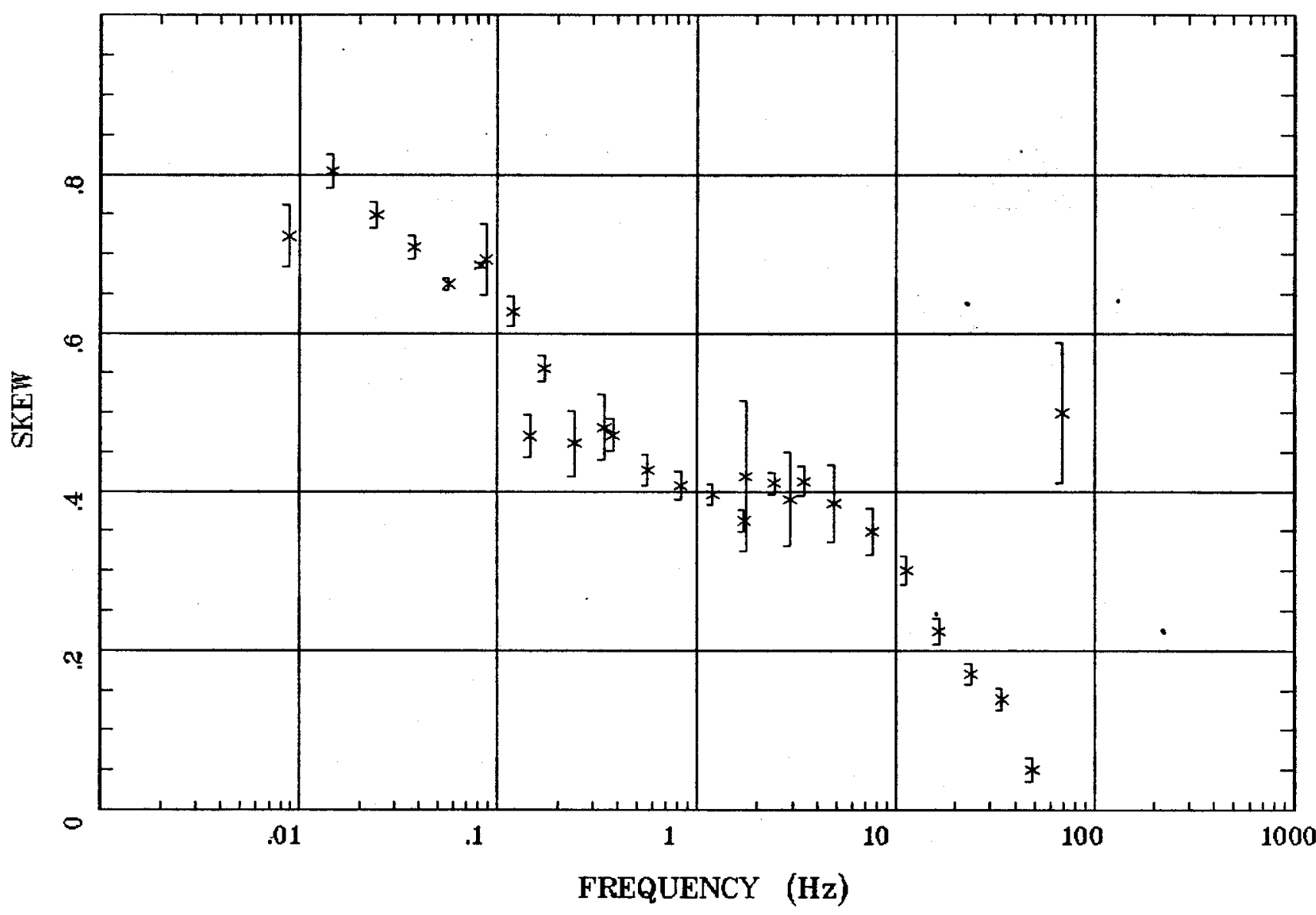

Client:

Remote: e-fld N $90 \mathrm{~m}$

Acquired: 10:2 Sep 18, 2003

Survey Co:USGS
Rotation:

Filename: ap24mall.avg

Channels: Ch1 Ch2 Ch3 Ch4 Ch5 Ch6 Ch7

Plotted: 11:25 Sep 24, 2003

$<$ EMI - ElectroMagnetic Instruments 


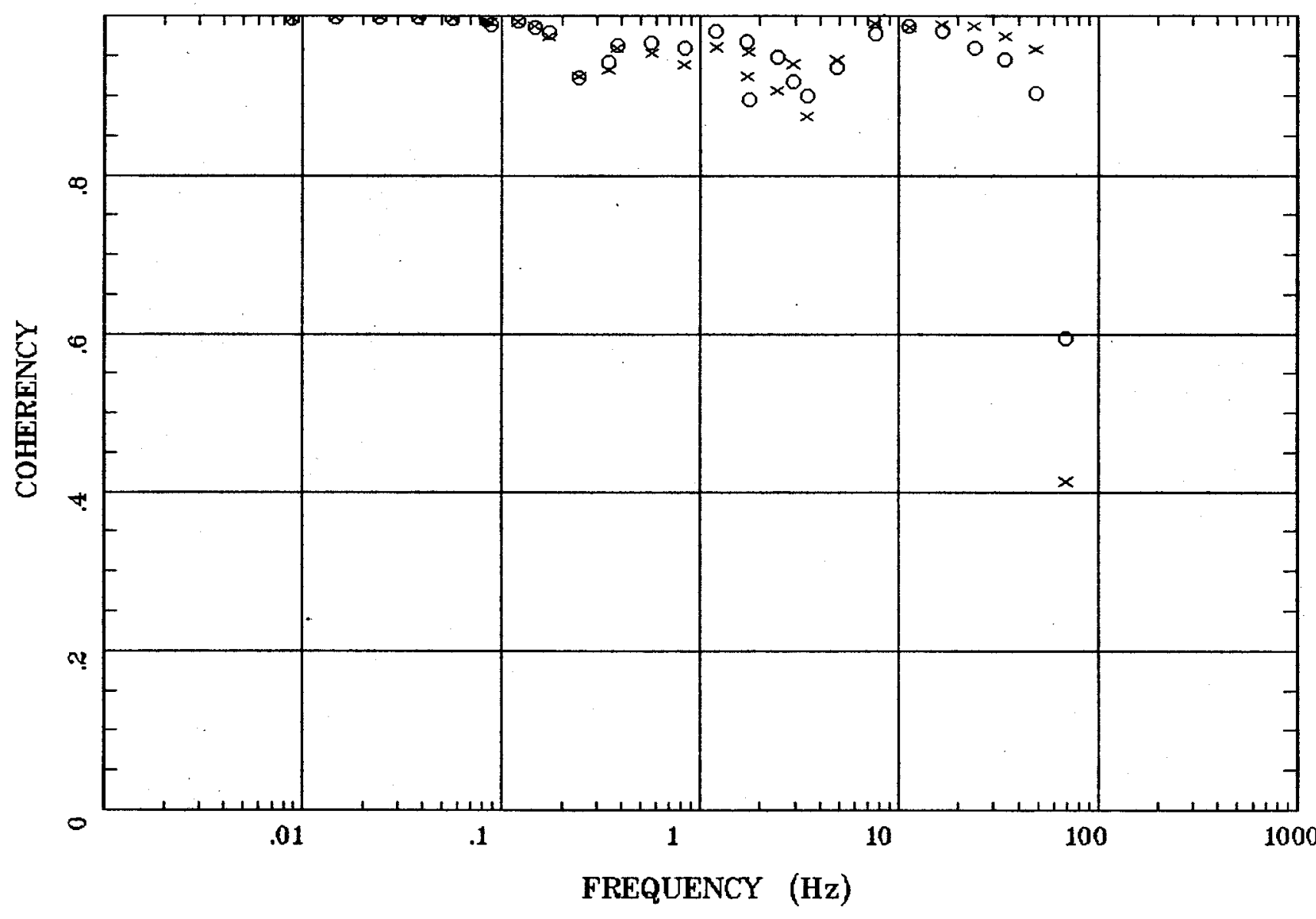

Client:

Remote: e-fld N $90 \mathrm{~m}$

Acquired: 10:2 Sep 18, 2003 Survey Co:USGS
Rotation:

Filename: ap24mall.avg

Channels: Ch1 Ch2 Ch3 Ch4 Ch5 Ch6 Ch7

Plotted: 11:25 Sep 24, 2003

$<$ EMI - ElectroMagnetic Instruments 


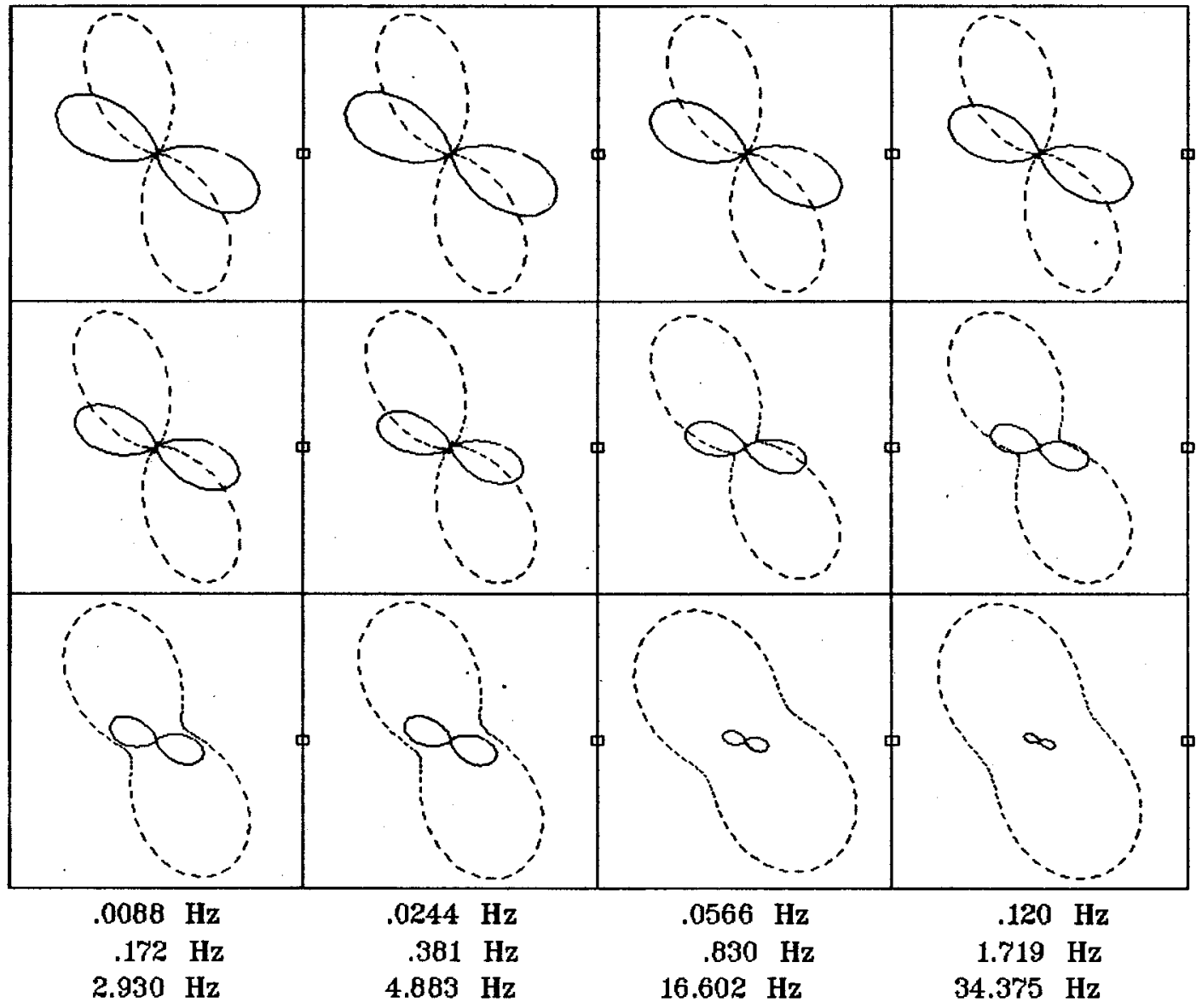

Rotation:

Client:

Remote: e-fld N $90 \mathrm{~m}$

Acquired: 10:2 Sep 18, 2003 Survey Co:USGS
Filename: ap24mall.avg

Channels: Ch1 Ch2 Ch3 Ch4 Ch5 Ch6 Ch7 Plotted: 11:26 Sep 24, 2003

< EMI - ElectroMagnetic Instruments > 


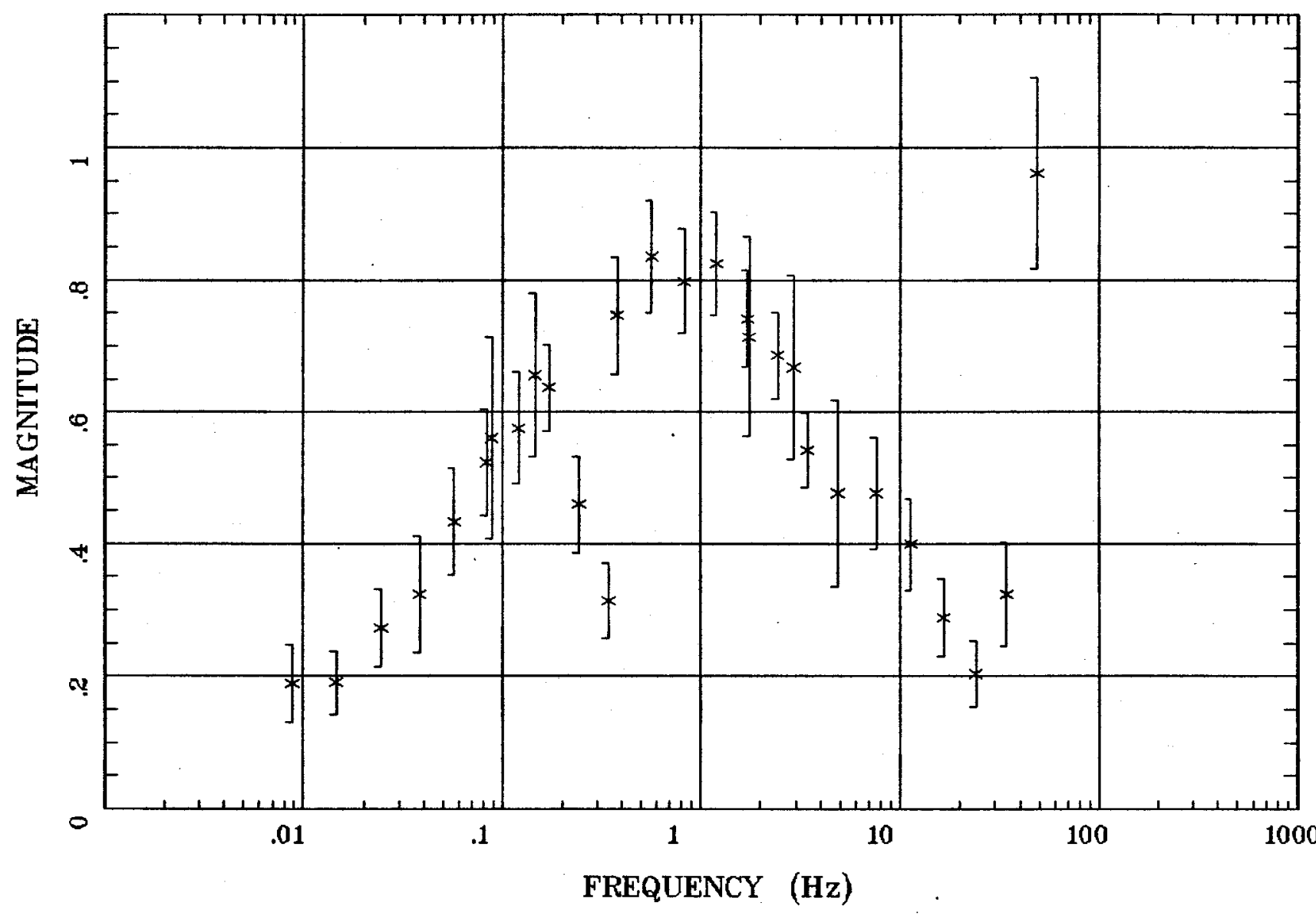

Client:

Remote: e-fld N $90 \mathrm{~m}$

Acquired: 10:2 Sep 18, 2003 Survey Co:USGS
Rotation:

Filename: ap24mall.avg

Channels: Ch1 Ch2 Ch3 Ch4 Ch5 Ch6 Chr Plotted: 11:26 Sep 24, 2003

$<$ EMI - ElectroMagnetic Instruments 


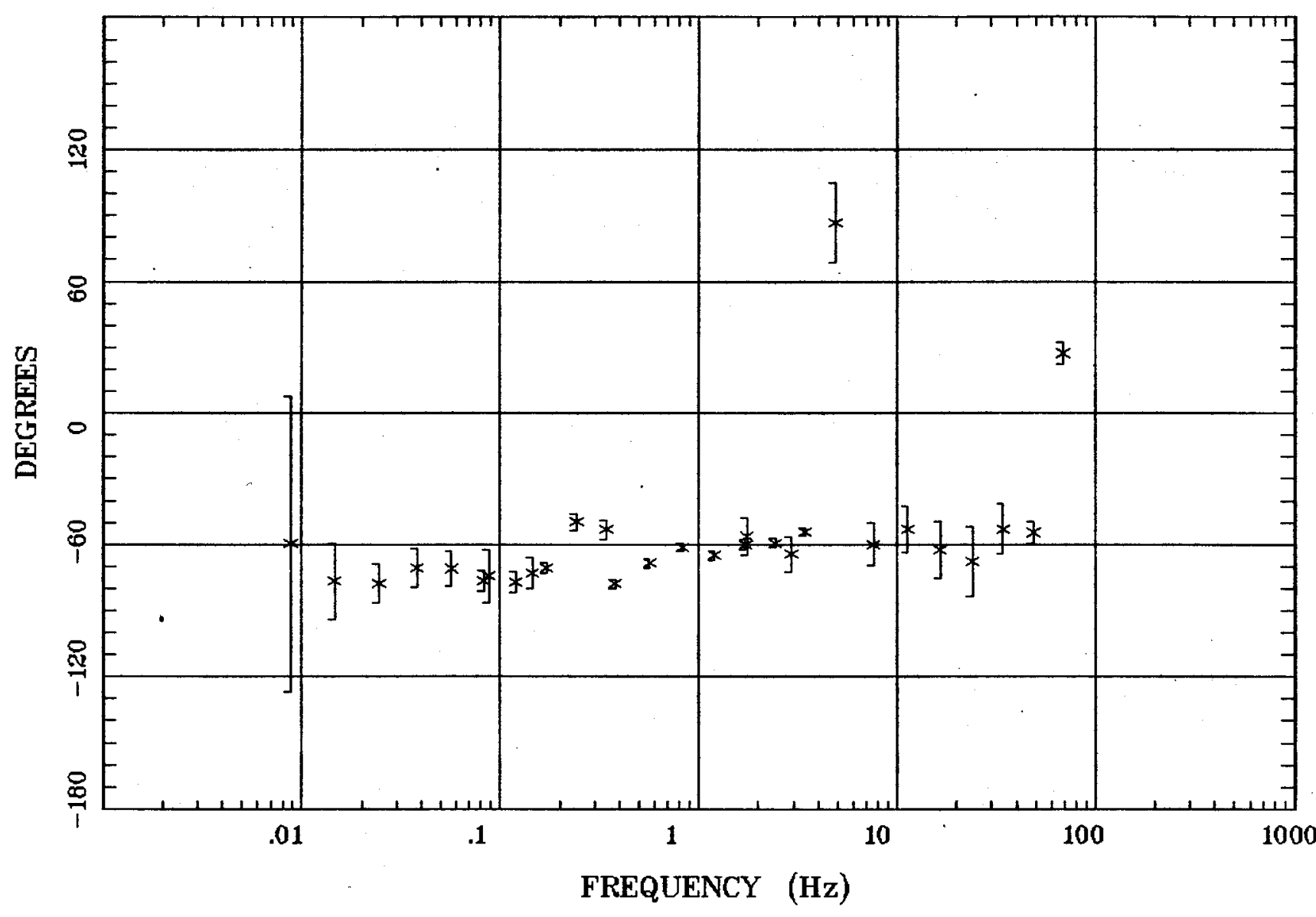

Client:

Remote: e-fld N $90 \mathrm{~m}$

Acquired: 10:2 Sep 18, 2003 Survey Co:USGS
Rotation:

Filename: ap24mall.avg Channels: Ch1 Ch2 Ch3 Ch4 Ch5 Ch6 Ch7 Plotted: 11:26 Sep 24, 2003

$<$ EMI - ElectroMagnetic Instruments 


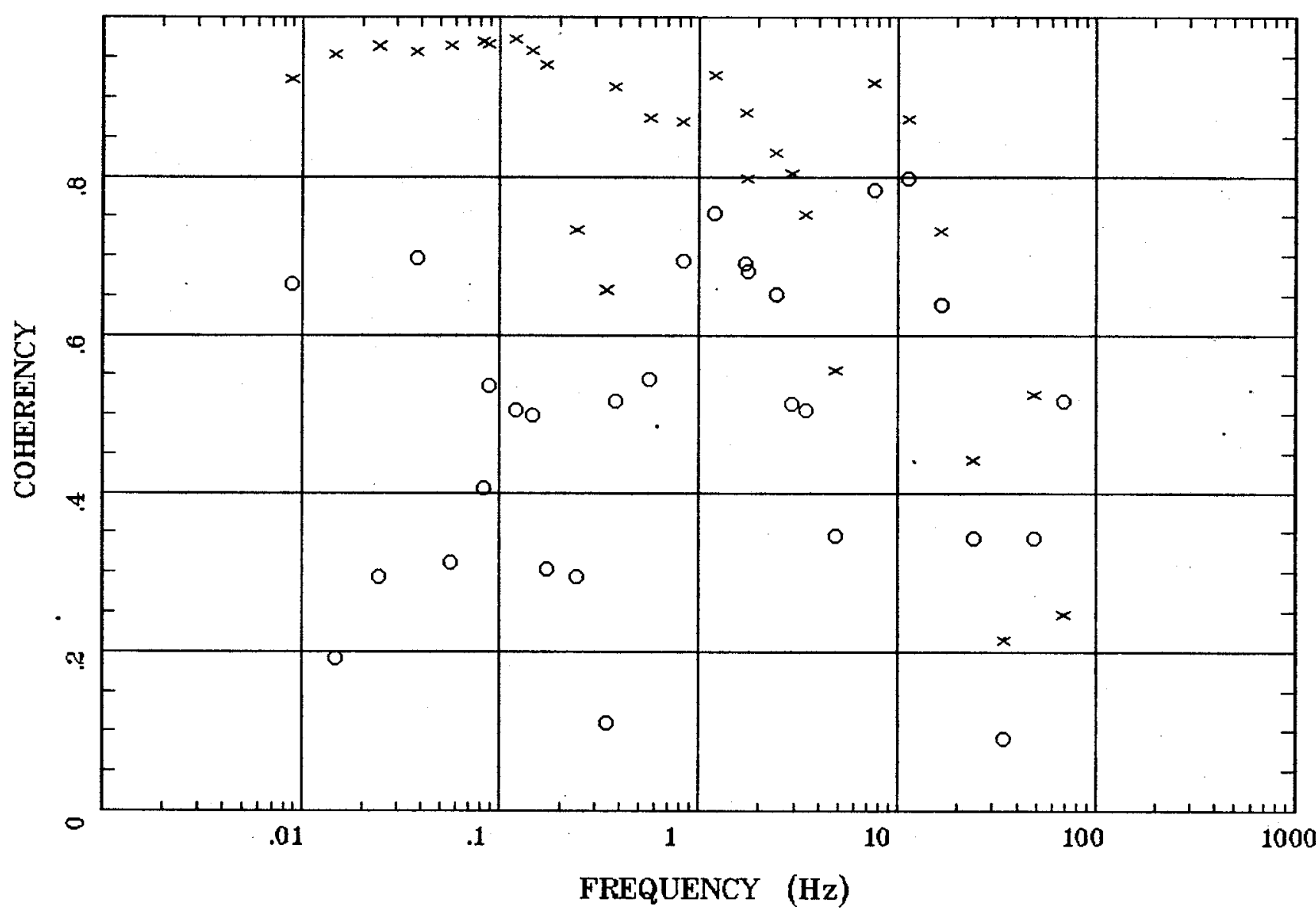

Client:

Remote: e-fld N $90 \mathrm{~m}$ Acquired: 10:2 Sep 18, 2003 Survey Co:USGS
Rotation:

Filename: ap24mall.avg

Channels: Ch1 Ch2 Ch3 Ch4 Ch5 Ch6 Ch7 Plotted: 11:26 Sep 24, 2003

< EMI - ElectroMagnetic Instruments 


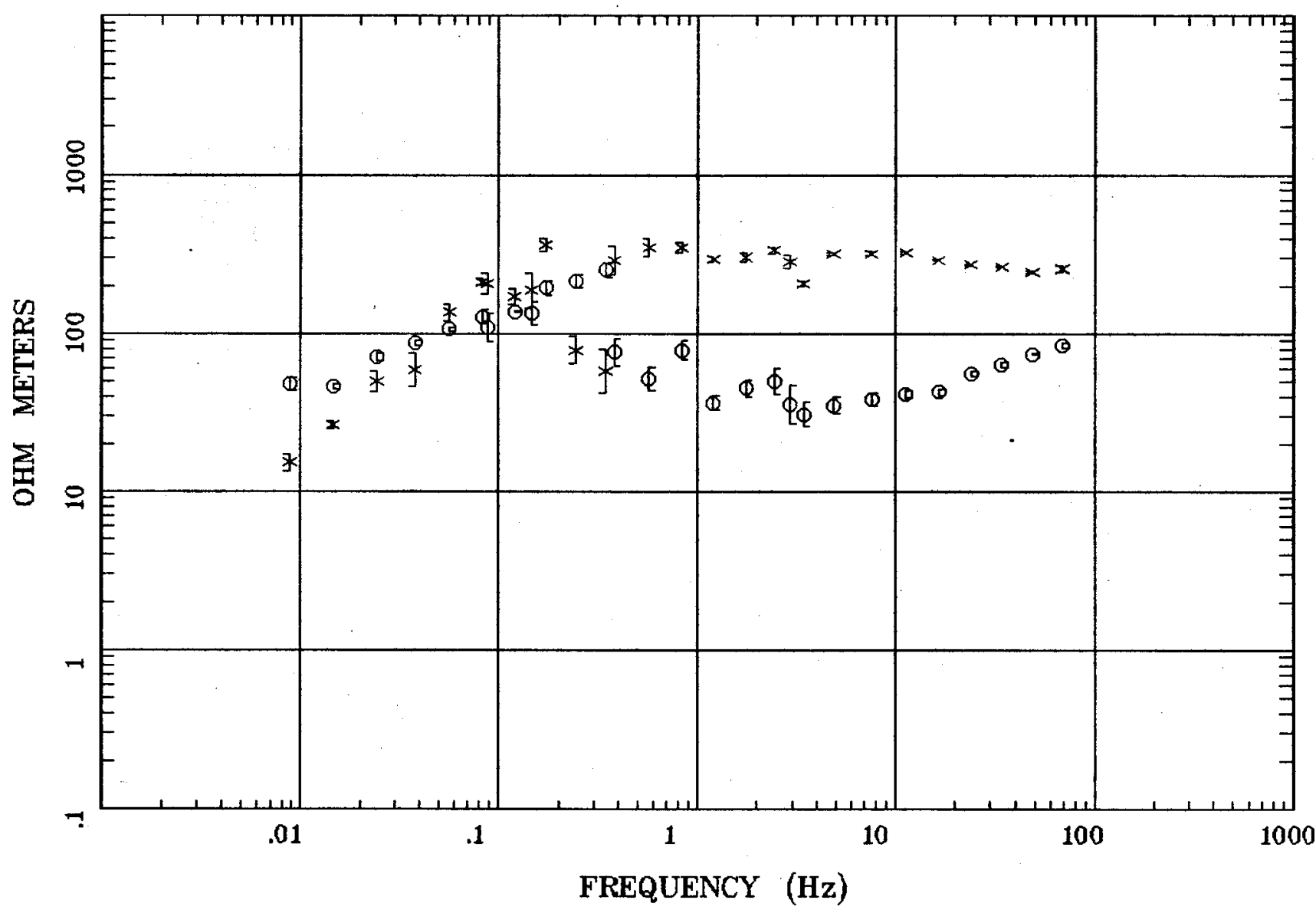

Client:

Remote: e-fld N $90 \mathrm{~m}$

Acquired: 14:4 Sep 18, 2003

Survey Co:USGS
Rotation:

Filename: ap25m.avg

Channels: Ch1 Ch2 Ch3 Ch4 Ch5 Ch6 Ch7

Plotted: 11:26 Sep 24, 2003

$<$ EMI - ElectroMagnetic Instruments > 


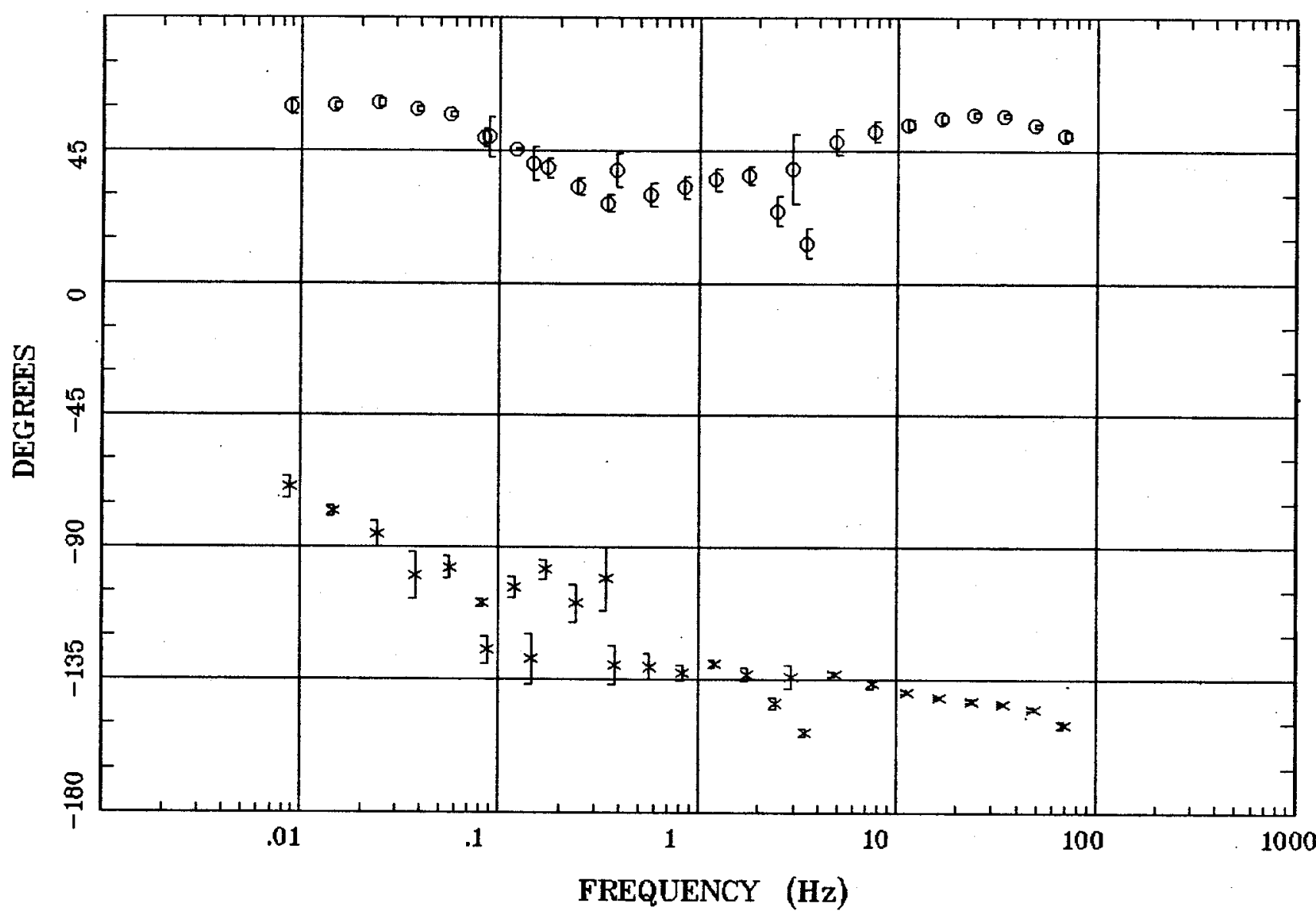

Client:

Remote: e-fld N $90 \mathrm{~m}$ Acquired: 14:4 Sep 18, 2003 Survey Co:USGS
Rotation:

Filename: ap25m.avg

Channels: Ch1 Ch2 Ch3 Ch4 Ch5 Ch6 Ch7 Plotted: 11:26 Sep 24, 2003

< EMI - ElectroMagnetic Instruments 
Station 25

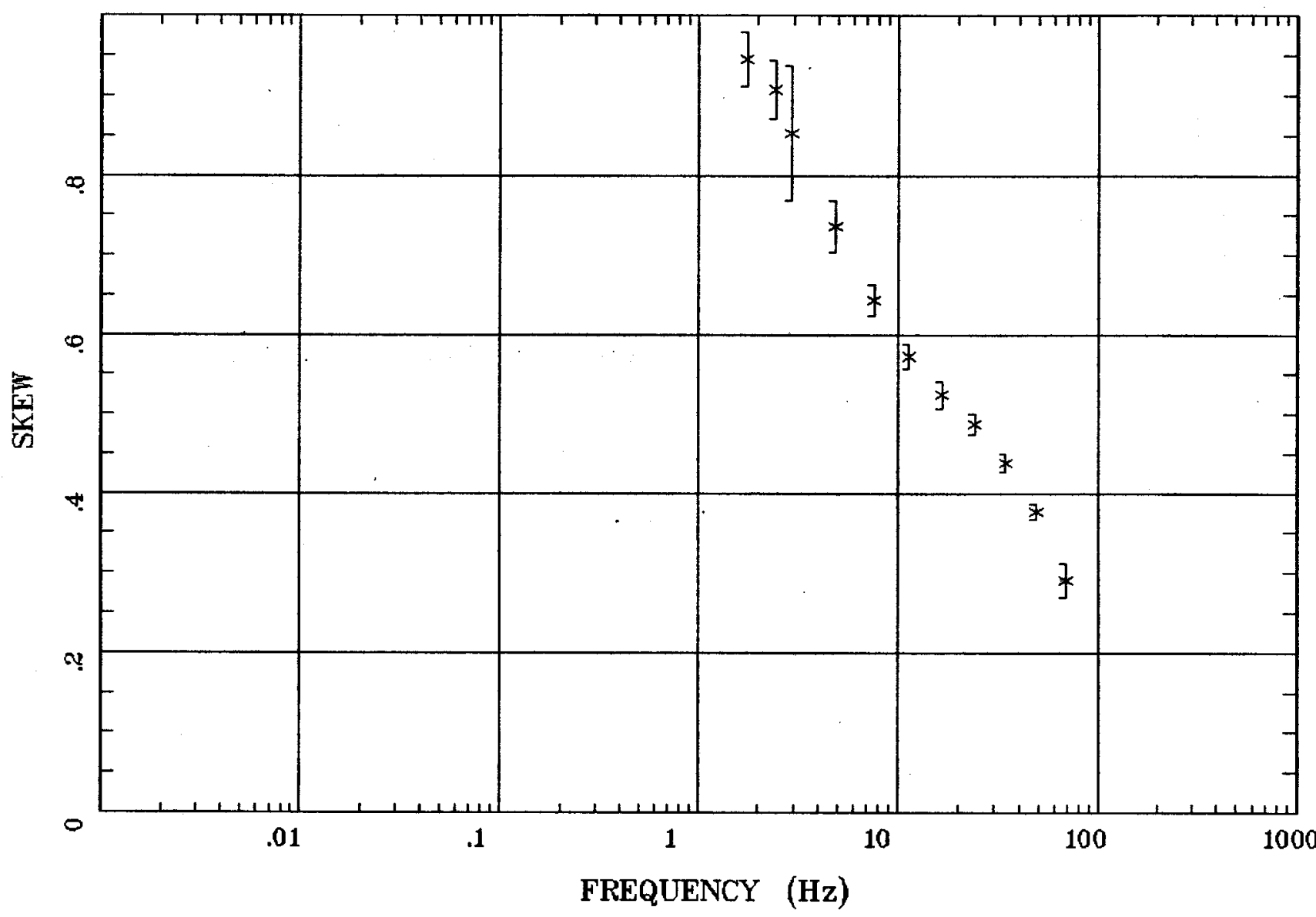

Client:

Remote: e-fld N 90m

Acquired: 14:4 Sep 18, 2003

Survey Co:USGS
Rotation:

Filename: ap25m.avg

Channels: Ch1 Ch2 Ch3 Ch4 Ch5 Ch6 Ch7

Plotted: 11:26 Sep 24, 2003

< EMI - ElectroMagnetic Instruments 
E MULT Coh.

Garrison, NV 100k Station 25

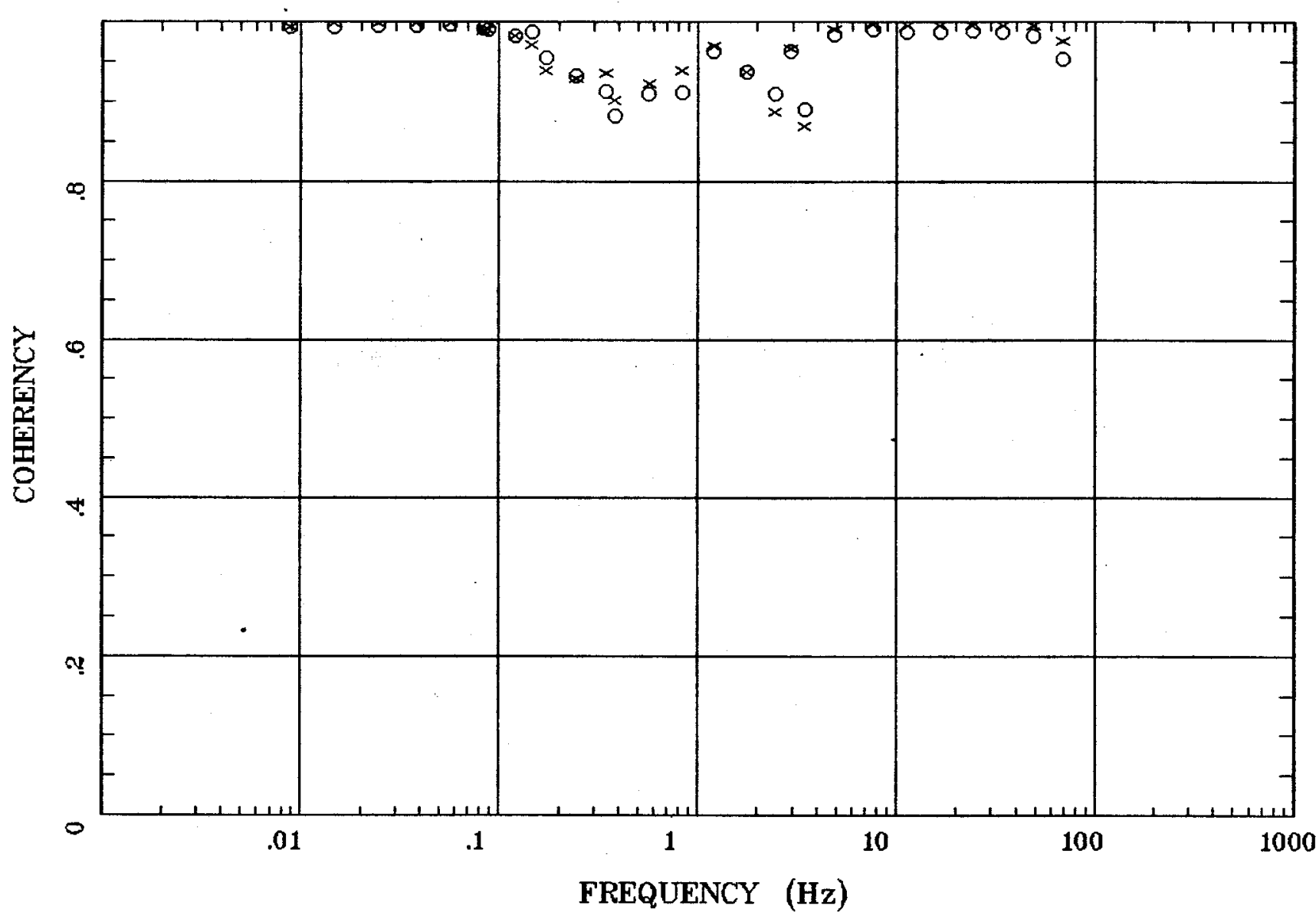

Client:

Remote: e-fld N $90 \mathrm{~m}$

Acquired: 14:4 Sep 18, 2003

Survey Co:USGS
Rotation:

Filename: ap25m.avg

Channels: Ch1 Ch2 Ch3 Ch4 Ch5 Ch6 Ch7

Plotted: 11:27 Sep 24, 2003

< EMI - ElectroMagnetic Instruments 


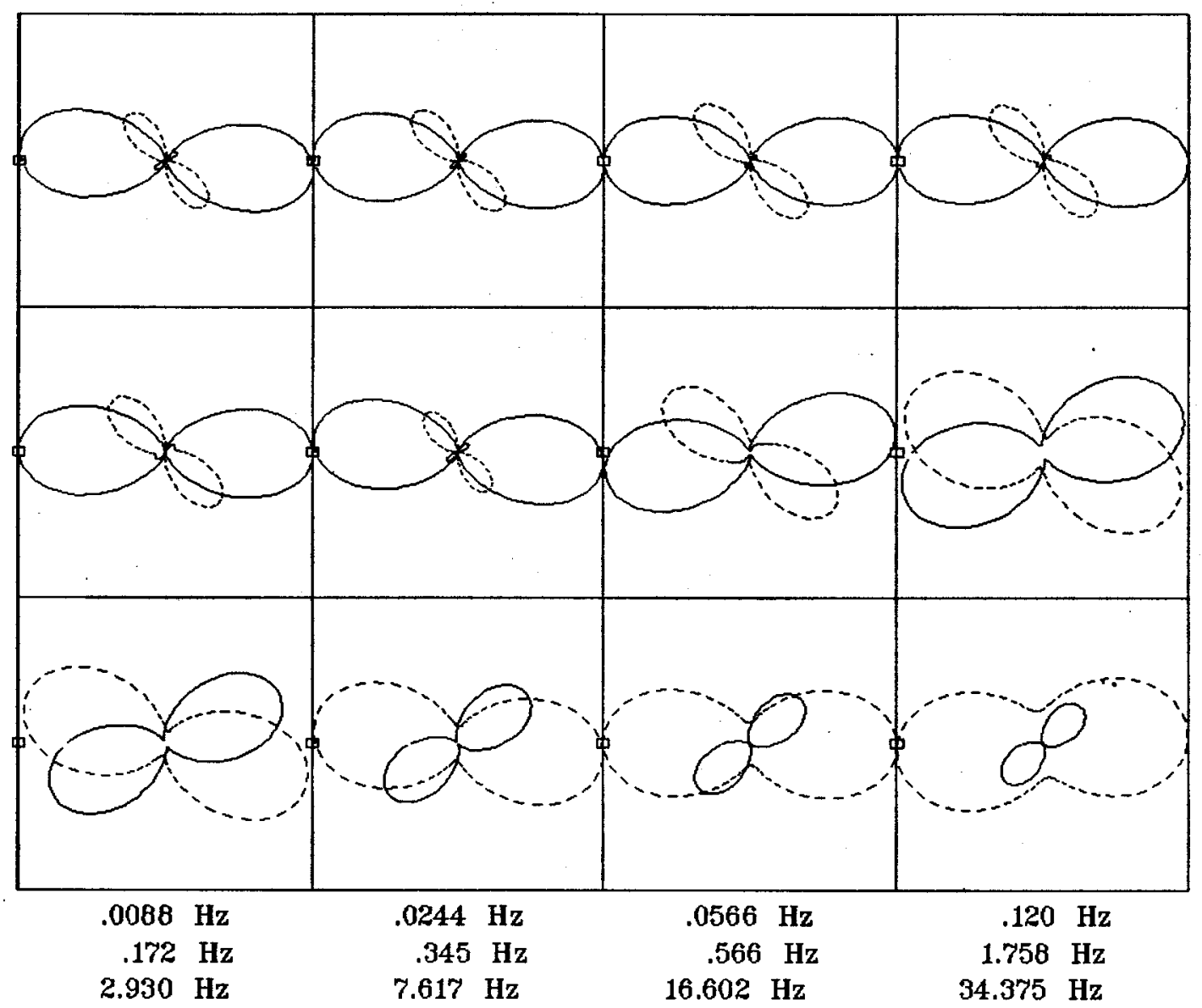

Client:

Remote: e-fld N $90 \mathrm{~m}$

Acquired: 14:4 Sep 18, 2003 Survey Co:USGS
Rotation:

Filename: ap25m.avg

Channels: Ch1 Ch2 Ch3 Ch4 Ch5 Ch6 Ch7

Plotted: 11:27 Sep 24, 2003

$<$ EMI - ElectroMagnetic Instruments > 


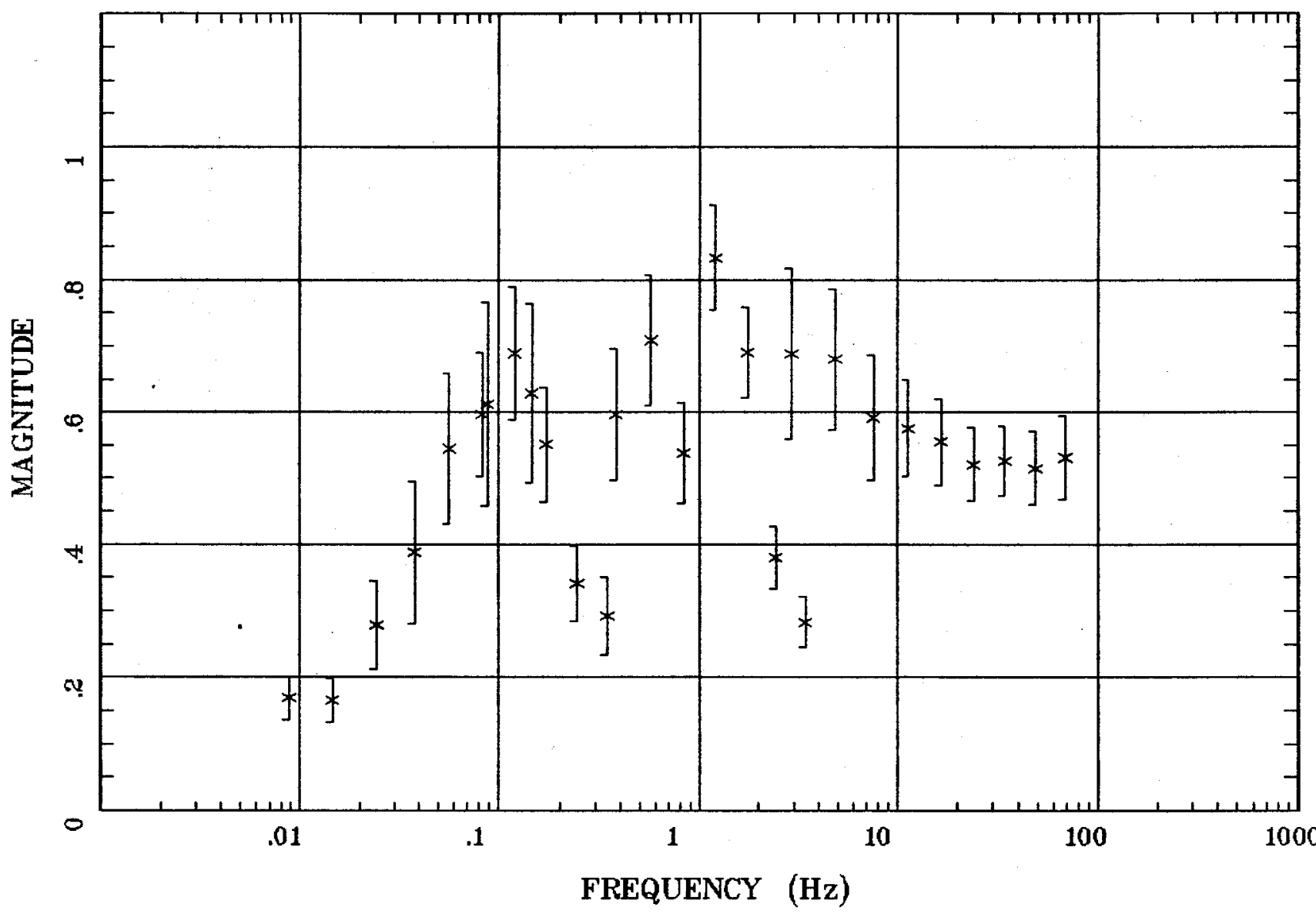

Client:

Remote: e-fld N $90 \mathrm{~m}$

Acquired: 14:4 Sep 18, 2003

Survey Co:USGS
Rotation:

Filename: ap25m.avg

Channels: Ch1 Ch2 Ch3 Ch4 Ch5 Ch6 Ch7

Plotted: 11:27 Sep 24, 2003

< EMI - ElectroMagnetic Instruments 


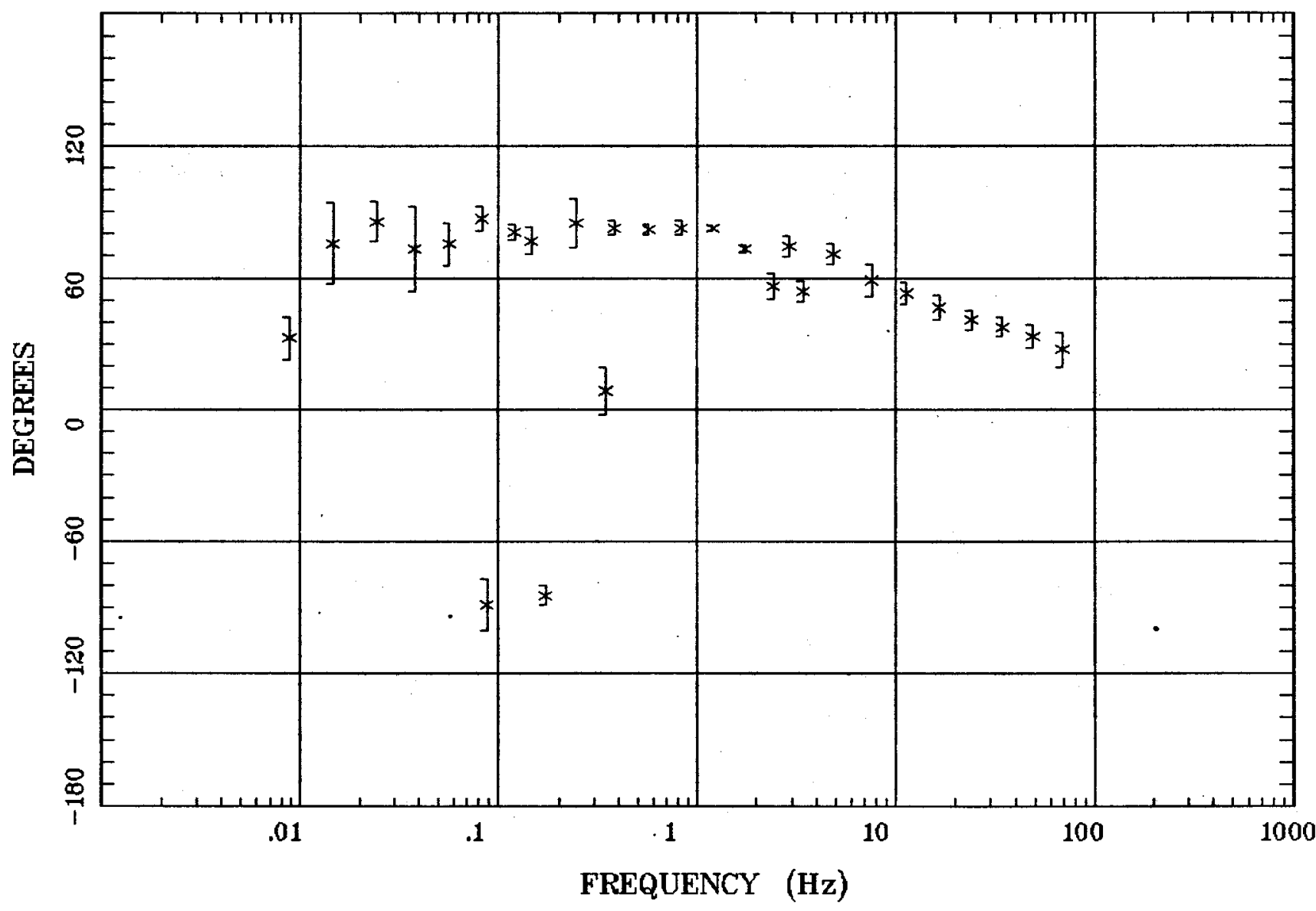

Client:

Remote: e-fld N $90 \mathrm{~m}$

Acquired: 14:4 Sep 18, 2003 Survey Co:USGS
Rotation:

Filename: ap25m.avg

Channels: Ch1 Ch2 Ch3 ch4 ch5 Ch6 Ch7 Plotted: 11:27 Sep 24, 2003

< EMI - ElectroMagnetic Instruments 


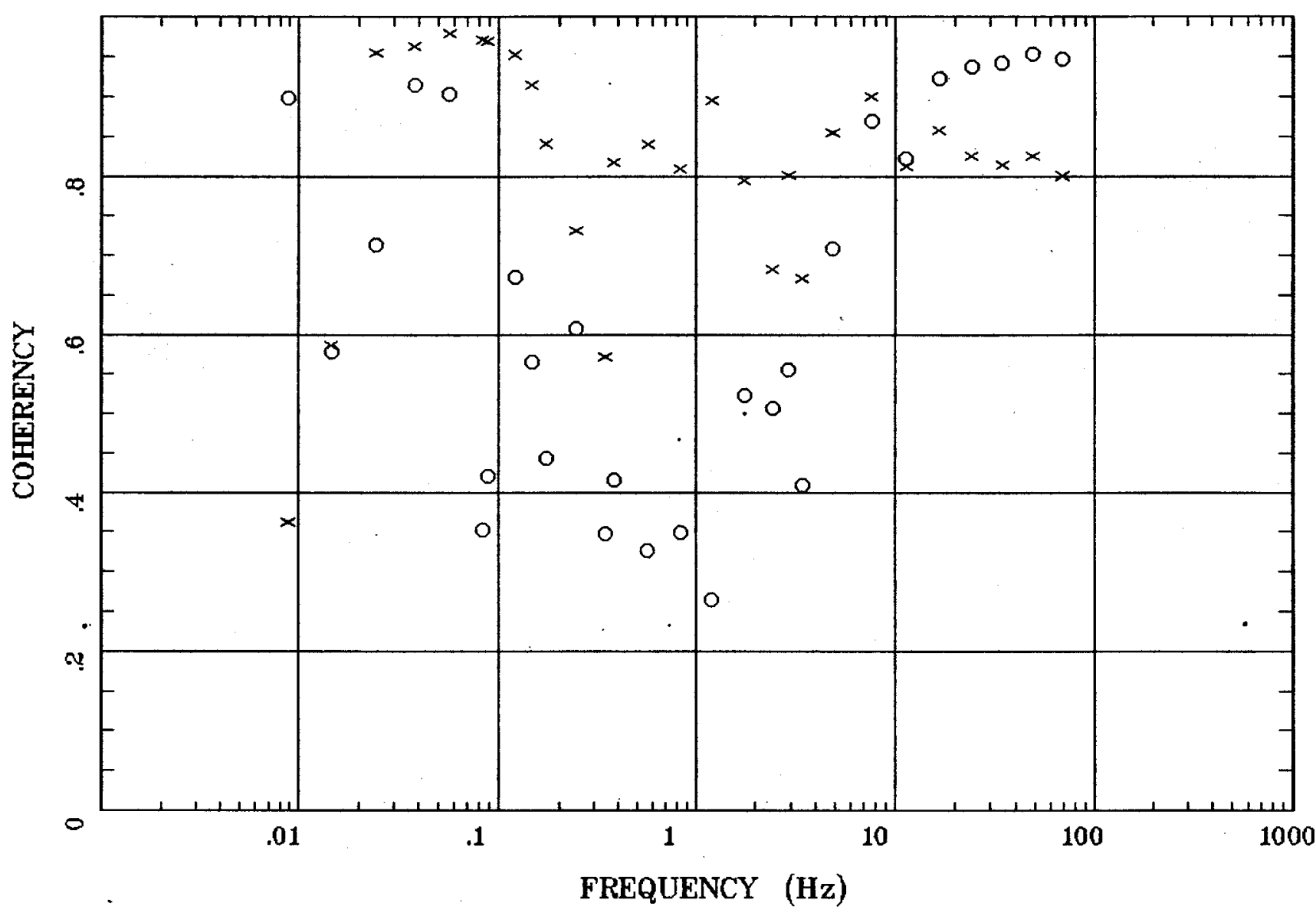

Client:

Remote: e-fld N $90 \mathrm{~m}$ Acquired: 14:4 Sep 18, 2003 Survey Co:USGS
Rotation:

Filename: ap25m.avg

Channels: Ch1 Ch2 Ch3 Ch4 Ch5 Ch6 Ch7 Plotted: 11:27 Sep 24, 2003

< EMI - ElectroMagnetic Instruments > 